\title{
SEARCHING FOR NEW MILLISECOND PULSARS WITH THE GBT IN FERMI UNASSOCIATED SOURCES
}

\author{
Siraprapa Sanpa-arsa \\ Khon Kaen, Thailand
}

B.A. Physics, Kasetsart University, 2009

M.S. Astronomy, University of Virginia, 2011

A Dissertation Presented to the Graduate

Faculty of the University of Virginia

in Candidacy for the Degree of

Doctor of Philosophy

Department of Astronomy

University of Virginia

July 2016

Committee Members:

Prof. Scott M. Ransom

Vanderbilt Prof. Craig Sarazin

VITA Assoc. Prof. Phil Arras

Prof. Brad Cox 
(c) Copyright by

Siraprapa Sanpa-arsa

All rights reserved

July 13, 2016 


\begin{abstract}
The launch of the Fermi satellite in 2008 revolutionized $\gamma$-ray pulsar astronomy by enabling the discovery of many new millisecond pulsars (MSPs). The Fermi Pulsar Search Consortium (PSC) has organized hundreds of radio observations of pulsar-like Large Area Telescope (LAT) unassociated sources. Over the past seven years, the PSC has discovered more than 70 new MSPs, compared to the 75 MSPs found in the 25 years prior to Fermi. The National Radio Astronomy Observatory's Robert C. Byrd Green Bank Telescope (GBT) has played the key role in the project by discovering almost half (34) of the new MSPs. In this thesis, I present the discovery and analysis of 16 new MSPs, 10 of which were uncovered by me personally. The pulsars were found in GBT searches within the positional error boxes of 266 Fermi LAT sources, both at high Galactic latitudes and closer to the Galactic plane. All new pulsars have phase-connected radio timing solutions, and for 12 of them, $\gamma$-ray pulsations were detected. Twelve MSPs have Helium white dwarf (He-WD) companions and the other four are in so-called "spider" systems with compact orbits and non-degenerate companions. We investigated the relationship between radio and $\gamma$-ray flux densities for all MSPs, confirming that there is almost no correlation between the two. We also investigated the orbital period vs. companion mass relation for MSPs with He-WD companions using a simple Monte Carlo technique, and found that the distribution of binary inclination angles is not random but possibly leans towards lower inclinations. For the four MSPs in compact orbits, we examined flux density variability, as well as their optical light curves. We found that all four MSPs are eclipsing and that two of them exhibit strong diffractive scintillation. Finally, we found optical counterparts for two MSPs, one of which shows ellipsoidal modulations in its light curve, suggesting that the companion is filling its Roche lobe.
\end{abstract}




\section{Acknowledgements}

I owe my deepest gratitude to my advisor, Prof. Scott Ransom. He is not only a rock climber, a socializer or a hyper pulsar-enthusiastic scientist, he is my very first mentor. Without his guidance, encouragement and support, this thesis would not have been complete. I am deeply thankful to my Fermi colleagues and collaborators who have been doing incredible science and inspired me to do great science like they do. I would like to express my gratitude to Tyrel Johnson who wrote the $\gamma$-ray analysis in Section 2.3.4, 2.4.2, 2.4.3, and provided Figure 2.2 in Chapter 2. Scott Ransom who wrote the joint $\gamma$-ray and radio timing and the $P_{b}-M_{c}$ relation in Section 2.3.5, 2.5.5 , and provided Figure 2.7 in Chapter 2. Anna Bilous who wrote the single-pulse search in Section 3.3.3, and provided Figure 3.10 and 3.11 in Chapter 3. Paul Ray who provided Figure 3.7 in Chapter 3. Jules Halpern who provided Figure 3.3, 3.5 and 3.8 in Chapter 3

I would like to thank my second family in UVA and friends who have been with me through the ups and downs in this journey. I love you guys more than words can ever express. Yes, that was very cliché. Lastly, this thesis and my life would not have existed without my family. They give me support, unconditional love and spread their wings to catch me whenever I fall. I could never ask for a better family. 


\section{Table of contents}

$\begin{array}{ll}\text { Abstract } & \text { ii }\end{array}$

1 Introduction 1

1.1 Pulsars . . . . . . . . . . . . . . . . . . . . . 1

1.1.1 Pulsar Overview . . . . . . . . . . . . . . . 1

1.1 .2 Pulsar properties . . . . . . . . . . . . . . . . . 4

1.1 .3 Millisecond pulsars . . . . . . . . . . . . . . . . . . 9

1.1 .4 Pulsar timing . . . . . . . . . . . . . . . . . 11

1.2 Searching for New Pulsars . . . . . . . . . . . . . . . . . . . . . 19

1.2 .1 RFI removal . . . . . . . . . . . . . . . . . . . . 20

1.2 .2 Dispersion Measure trials . . . . . . . . . . . . . . . . . 21

1.2 .3 Periodic Searches . . . . . . . . . . . . . . . . . . . 21

1.2.4 Acceleration Search . . . . . . . . . . . . . . . . . . . . . 22

1.2 .5 Candidate selection . . . . . . . . . . . . . . . . 23

1.2 .6 Single-pulse search . . . . . . . . . . . . . . . . . . 23

1.3 Fermi Unassociated Sources . . . . . . . . . . . . . . . . . . . . . 25

1.3.1 Fermi satellite . . . . . . . . . . . . . . . 25

1.3.2 Fermi "Treasure Map" . . . . . . . . . . . . . . . . . . 27

1.3.3 The Pulsar Search Consortium (PSC) . . . . . . . . . . . . 28

1.4 Pulsar Search with the Green Bank Telescope (GBT) . . . . . . . . . 31

2 Discovery of Twelve New Millisecond Pulsars in Fermi LAT Sources with the Green Bank Telescope 33

2.1 Introduction . . . . . . . . . . . . . . . . . . . . . 33

2.2 Source Selection . . . . . . . . . . . . . . . . . . . . . . 35

2.3 Observation and Data Analysis . . . . . . . . . . . . . 38

2.3.1 Observation Method and Sensitivity . . . . . . . . . . . . 38

2.3.2 Pulsar Search Method . . . . . . . . . . . . . . . . . . . . 39

2.3 .3 Pulsar Timing . . . . . . . . . . . . . . . . . . . . . . . . . 42

2.3.4 LAT Data Analysis . . . . . . . . . . . . . . . . . 45

2.3.5 Joint $\gamma$-ray and Radio Timing . . . . . . . . . . . . . . 46

2.4 Results . . . . . . . . . . . . . . . . . . . 48 
2.4.1 The New MSPs . . . . . . . . . . . . . . . . . . . . . . 48

2.4.2 Radio and Gamma-ray Light Curves . . . . . . . . . . . . . . 53

2.4.3 Gamma-ray Pulsations . . . . . . . . . . . . . . 56

2.5 Discussion . . . . . . . . . . . . . . . . . . . . . 59

2.5.1 Radio and $\gamma$-ray Flux Densities . . . . . . . . . . . . . . 59

2.5.2 Galactic Plane Searches with S-band . . . . . . . . . . . . . 60

2.5.3 Single-Pulse Searches . . . . . . . . . . . . . . . 62

2.5.4 LAT $\gamma$-ray Detection Threshold . . . . . . . . . . . . . . . . 62

2.5.5 Orbital Period and Companion Mass Relation . . . . . . . . 65

2.6 Conclusion . . . . . . . . . . . . . . . . . 70

3 Four New Pulsars in Tight Orbits 73

3.1 "Spider" Pulsars . . . . . . . . . . . . . . . . . . . 73

3.2 Observation and data analysis . . . . . . . . . . . . . . 75

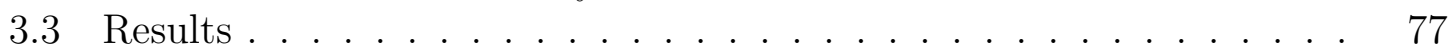

3.3.1 Pulsar timing . . . . . . . . . . . . . . . . . . . . . 77

3.3 .2 The new MSPs . . . . . . . . . . . . . . . . 78

3.3.3 Single-pulse search .................. 81

3.4 Discussion . . . . . . . . . . . . . . . . . . . . . . . . . . . . . . . . . . . . 82

3.4.1 Flux variations . . . . . . . . . . . . . . . . 82

3.4.2 Optical, X-ray and $\gamma$-ray counterparts . . . . . . . . . 83

3.5 Conclusion . . . . . . . . . . . . . . . . 84

4 Conclusions and Future Work 101

5 Appendix: Tables 104 


\section{Chapter 1}

\section{Introduction}

\subsection{Pulsars}

\subsubsection{Pulsar Overview}

The prediction of the existence of neutron stars, the smallest and densest stars, had been made before they were actually discovered. In 1934, the two astronomers, Walter Baade and Fritz Zwicky, wrote in Baade \& Zwicky (1934) that: "With all reserve we advance the view that a super-nova represents the transition of an ordinary star into a neutron star, consisting mainly of neutrons. Such a star may possess a very small radius and an extremely high density." More than 30 years later, in 1967, graduate student Jocelyn Bell recognized regular fluctuations of a signal from a radio source. Together with her advisor, Antony Hewish, they found that the signals peaked every 1.34 seconds and reappeared once every sidereal day, which suggested a celestial (outside the Solar System) origin. In 1968 they announced that the repeated radio signals likely came from a rotating neutron star, the pulsar B1919+21 (Hewish et al. 1968). See Fig. 1.1. 

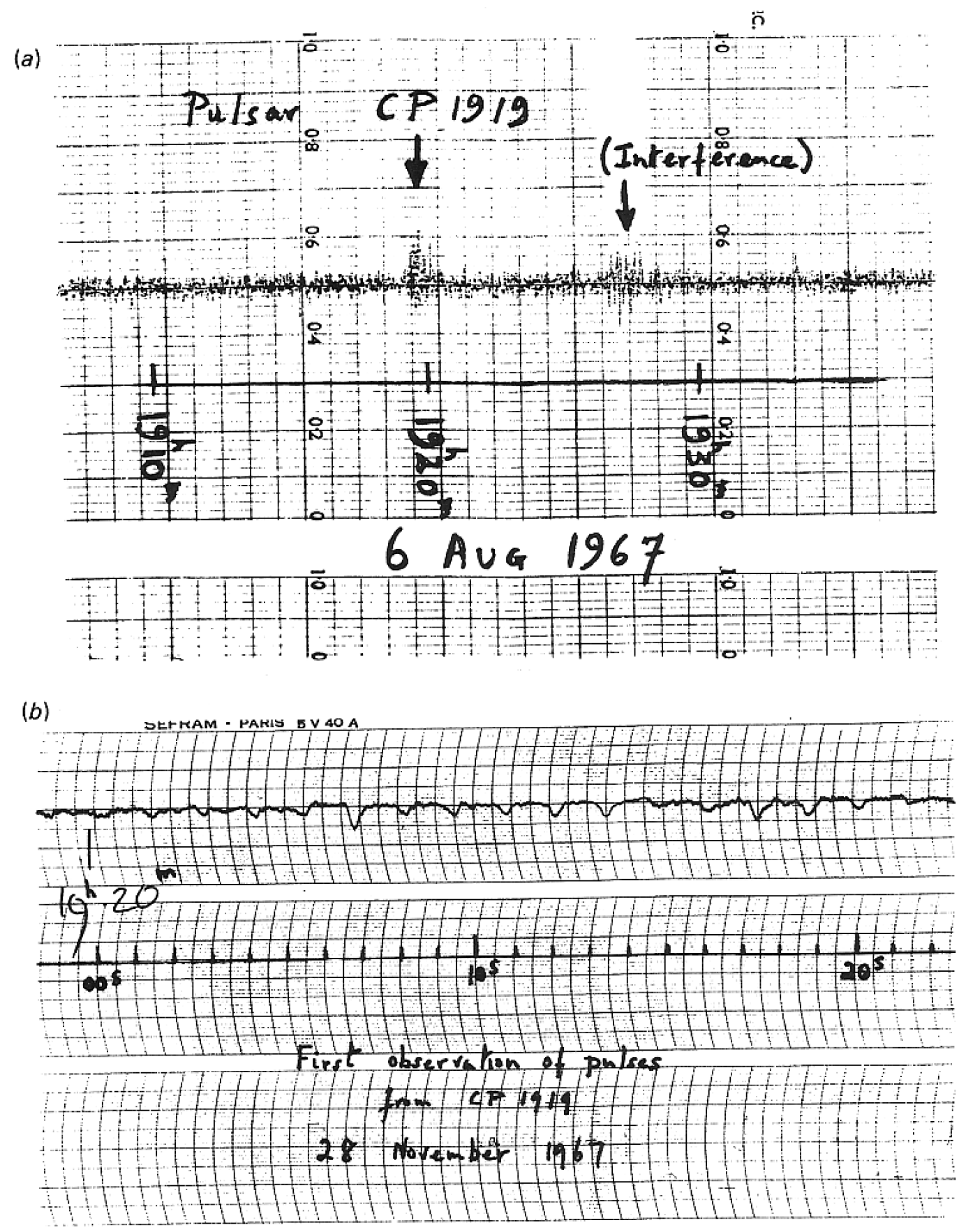

Fig. 1.1. - : The discovery of the first pulsar B1919+21. (a) The radio signals first appeared with the characteristics of radio interference. (b) Fast chart recordings showed repeated individual pulses at every $1.34 \mathrm{~s}$ (Hewish et al. 1968). 
Pulsars are highly magnetized, rapidly rotating, neutron stars, the final products of the supernovae of massive stars $\left(\sim 8-20\right.$ times the mass of our sun, $\left.M_{\odot}\right)$. As these incredibly dense objects $\left(\sim 1-2 M_{\odot}\right.$, but only about $20 \mathrm{~km}$ in diameter $)$ rotate, they emit beams of radiation, producing pulses each time the beams sweep across an observer's line of sight, in a type of "light house effect". The precision and stability of pulsar rotations are incredible, due to their density, and they are therefore often called the clocks of the Universe.

In many aspects, pulsars are a "physicist's dream come true" (Lorimer \& Kramer 2005). They can be used to study physics under extreme conditions which do not exist on Earth such as theories of gravity in deep gravitational potentials and the exotic solid state and nuclear physics in the interiors ultra-dense neutron stars. Pulsars can furthermore be used to study the gravitational potential and magnetic field of the Galaxy, the interstellar medium (ISM), and binary systems and their often complex evolution. Pulsar timing allows us to precisely measure pulsar spins, astrometric parameters, and the effects of the ISM between the pulsar and the observer. The fast-rotating population, millisecond pulsars (MSPs), however, are much preferred and more useful for pulsar timing than the slow population of normal pulsars. MSP signals can be measured more precisely and they do not exhibit rotational instabilities which are common in normal pulsars. These properties make MSPs much better clocks which are more useful for exotic pulsar timing applications. This thesis deals primarily with MSPs. 


\subsubsection{Pulsar properties}

$P-\dot{P}$ diagram

As a pulsar evolves, its spin period $(P)$ gradually increases with time corresponding to a rate of "spin-down" $(\dot{P})$. This spin-down is a result of its loss of rotational kinetic energy which it emits in electromagnetic radiation and particles. The characteristic age $(\tau \propto P / \dot{P})$, the magnetic field strength $(B \propto \sqrt{P \dot{P}})$ and the rate of loss of rotational kinetic energy or "spin-down luminosity" $\left(\dot{E} \propto \dot{P} / P^{3}\right)$ of the pulsar can be determined with only the two observable parameters, $P$ and $\dot{P}$. The " $P-\dot{P}$ diagram" of pulsars shown in Fig. 1.2 therefore provides insight into the spin evolution of neutron stars. Note that $P$ and $\dot{P}$ of a pulsar can be obtained precisely via pulsar timing (see Section 1.1.4).

The $P-\dot{P}$ diagram clearly shows two distinct populations of pulsars: "normal pulsars" $\left(P \sim 0.5 \mathrm{~s}\right.$ and $\left.\dot{P} \sim 10^{-15} \mathrm{~s} \mathrm{~s}^{-1}\right)$ and "millisecond pulsars" $(P \sim 30 \mathrm{~ms}$ and $\left.\dot{P} \sim 10^{-20} \mathrm{ss}^{-1}\right)$. The lines of constant $\tau, B$ and $\dot{E}$ are also shown on the $P-\dot{P}$ diagram. A plausible evolutionary track for normal pulsars starts with their birth in supernovae in the middle and upper left region of the diagram. Assuming a constant $B$, pulsars gradually move down and right along the lines of constant $B$, crossing the lines of constant age as $P$ increases. On a timescale of $\sim 10^{7}$ years, either old pulsars' magnetic fields or spin rates are too low to produce radio emission, and they eventually become too faint to detect. However, through the "recycling process", an old (or dead) pulsar in a binary system can be spun-up by accreting mass and angular momentum from its companion and become detectable again. These recycled pulsars are the MSPs in the lower left of the $P-\dot{P}$ diagram. 


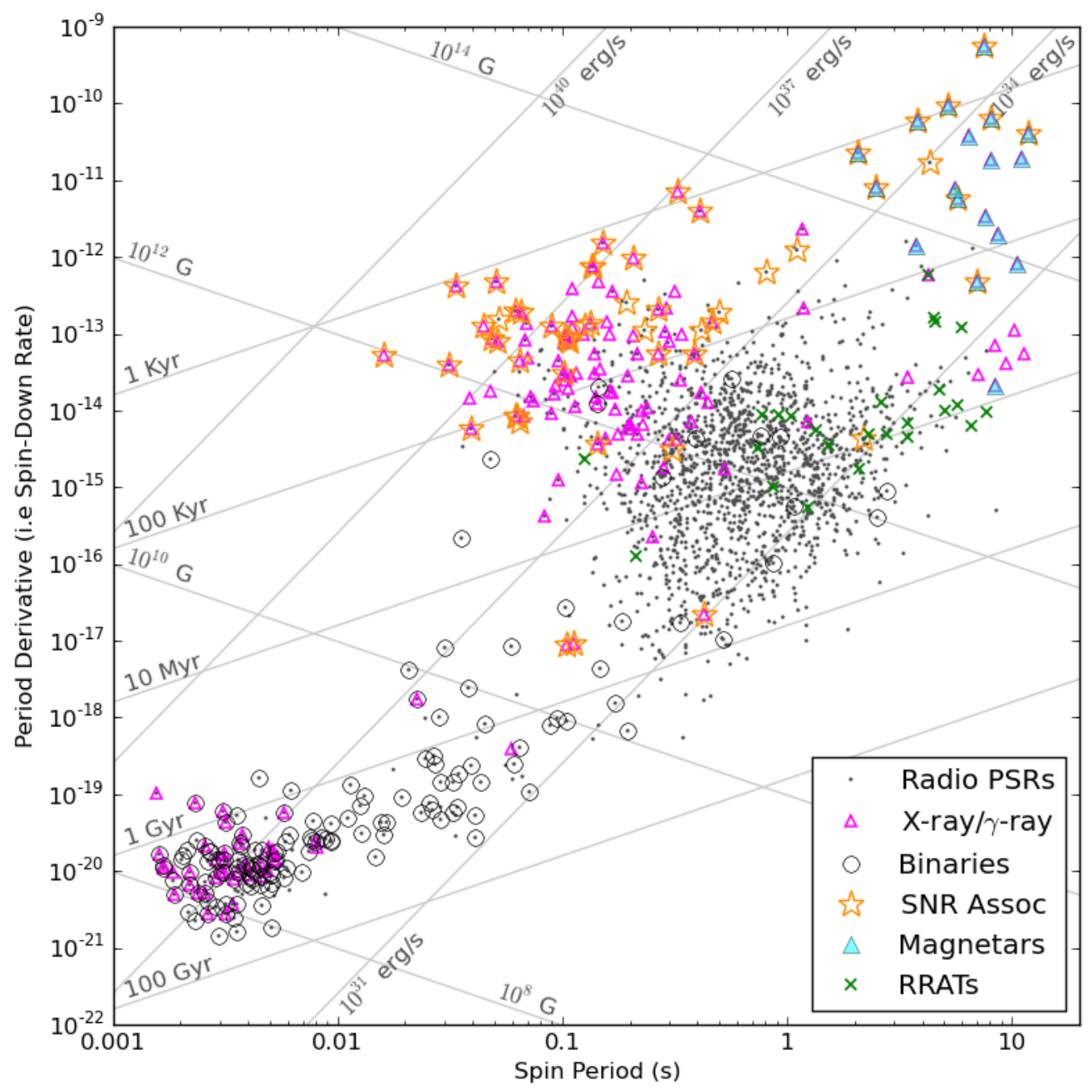

Fig. 1.2.- : The $P-\dot{P}$ diagram of over 2,200 pulsars. The majority of the pulsars, the "normal" pulsars, have spin period more than $0.3 \mathrm{~s}$ and appear on the middle right of the diagram. The millisecond pulsars have spin period less than $0.3 \mathrm{~s}$ and locate on the bottom left of the diagram. Image credit: Scott Ransom 


\section{Dispersion measure (DM) and de-dispersion}

As radio waves from pulsars travel through the ionized plasma in the interstellar medium (ISM), the radiation experiences a frequency-dependent dispersive effect pulses at lower frequencies travel through a plasma slower than the ones at higher frequencies. The time delay from the dispersion between two frequencies $(\Delta t)$ of the radio waves can be described by

$$
\left(\frac{\Delta t}{\mathrm{~s}}\right) \approx 4.15 \times 10^{3} \times\left[\left(\frac{f_{1}^{-2}}{\mathrm{MHz}}\right)-\left(\frac{f_{2}^{-2}}{\mathrm{MHz}}\right)\right] \times\left(\frac{\mathrm{DM}}{\mathrm{pc} \cdot \mathrm{cm}^{-3}}\right),
$$

and

$$
\mathrm{DM} \equiv \int_{0}^{d} n_{e} d l
$$

where $f$ is the frequency of the radio wave, DM is the "dispersion measure", $d$ is the distance to the pulsar, and $n_{e}$ is the electron number density. A known DM can be used to estimate the distance of the pulsar using the model of the electron density distribution in the Galaxy (e.g. NE2001 by Cordes \& Lazio 2002).

"Incoherent de-dispersion" is the simplest way to compensate for the dispersion of pulses. The observing frequency band is split into numerous independent frequency channels, and each channel is shifted in time by the delay calculated with Eq. 1.1 using the correct value of DM (See Fig. 1.3). As a result the pulses from each channel are made to arrive at the same time. Most of the observations in this thesis were processed using incoherent de-dispersion.

\section{Magnetic field strength}

A core-collapse supernova dramatically amplifies the magnetic field strength $(B)$ in

the core of the collapsing star, making that in the resulting neutron star incredibly 


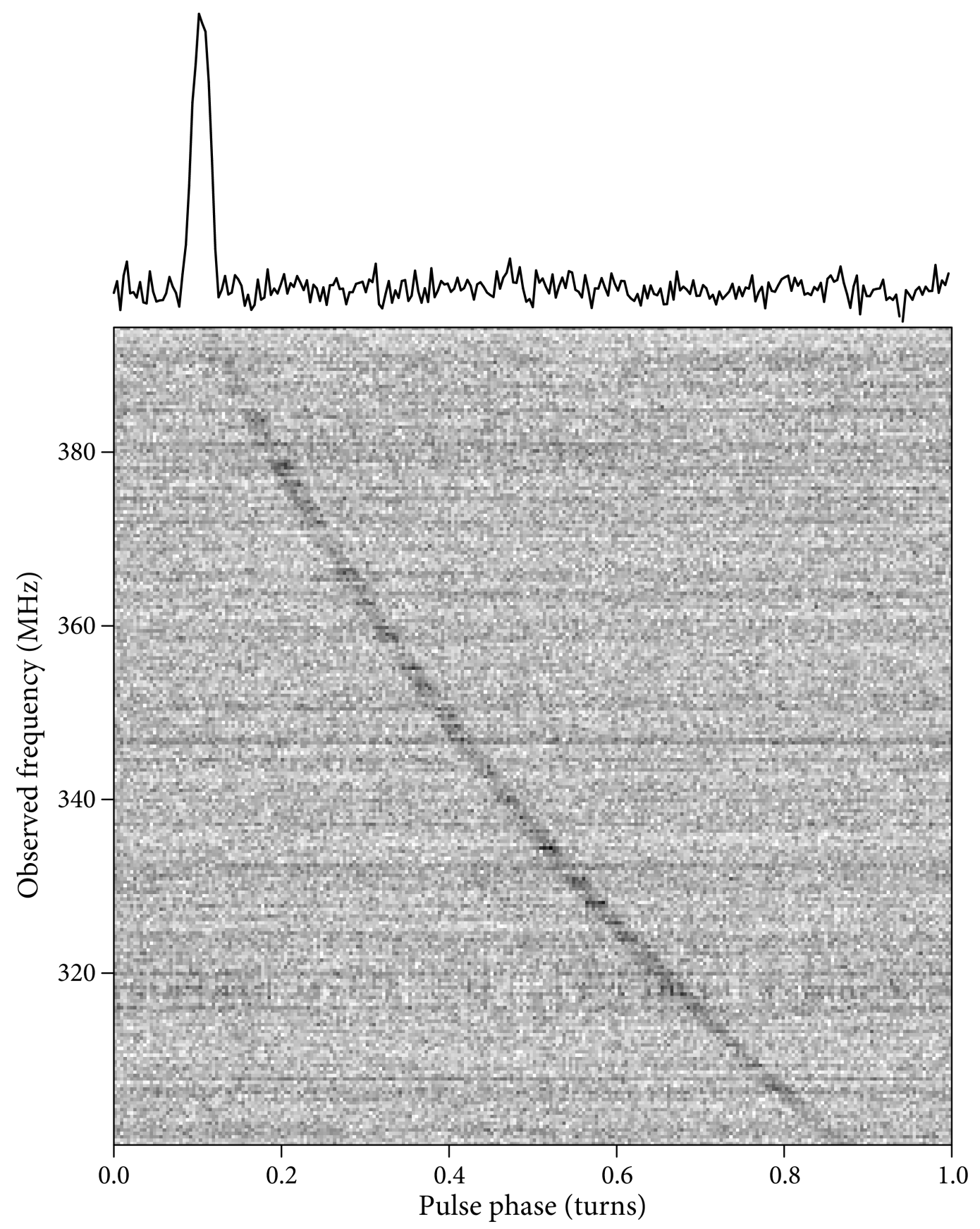

Fig. 1.3.-: The diagram shows pulse dispersion due to the ionized interstellar medium. The grey scale shows dispersive pulse delays of PSR J1400+50. The dedispersed integrated pulse profile in shown at the top of the diagram. Image credit: Scott Ransom 
large. From the conservation of magnetic flux $\left(\Phi \equiv \int \vec{B} \cdot \hat{n} d a\right.$ where $\hat{n}$ is a unit vector and $a$ is the surface area), a decrease in the radius of a star, for example, by a factor of 10 after collapsing, would decrease the surface area and therefore increase the magnetic strength by a factor of 100. Pulsars therefore have strong dipole magnetic fields.

The surface magnetic field strength $\left(B_{s}\right)$ of a radio pulsar, however, cannot be measured directly, but can be estimated by assuming a neutron star moment of inertia (I) of $10^{45} \mathrm{~g} \mathrm{~cm}^{2}$, a radius of $10 \mathrm{~km}$, that the magnetic moment and spin axis are perpendicular, and that the spin-down process is dominated by dipole braking:

$$
B_{s} \simeq 10^{12} \mathrm{G} \sqrt{\frac{\dot{P}}{10^{-15}} \cdot \frac{P}{\mathrm{~s}}}
$$

Note that this is the magnetic strength at the equator not at the poles.

\section{Spin-down luminosity}

The pulsar spin period decreases with time as a result of the loss of rotational kinetic energy $\left(E_{\text {rot }}\right)$. The rate of rotational kinetic energy or the "spin-down luminosity" $(\dot{E})$ can be estimated by assuming the canonical, $I$, of $10^{45} \mathrm{~g} \mathrm{~cm}^{2}$

$$
\dot{E} \equiv-\frac{\mathrm{d} E_{\mathrm{rot}}}{\mathrm{d} t}=-\frac{\mathrm{d}\left(I \Omega^{2} / 2\right)}{\mathrm{d} t}, \text { where } \Omega=2 \pi / P
$$

and with constants evaluated, and in useful units,

$$
\dot{E} \simeq 3.95 \times 10^{31} \mathrm{erg} \mathrm{s}^{-1}\left(\frac{\dot{P}}{10^{-15}}\right)\left(\frac{P}{\mathrm{~s}}\right)^{-3}
$$




\section{Characteristic age}

The pulsar age can be approximated with only two observables, $P$ and $\dot{P}$. Under the assumption that magnetic dipole radiation causes the spin-down and that the birth spin period is much smaller than the present one,

$$
\tau \equiv \frac{P}{2 \dot{P}},
$$

where $\tau$ is the "characteristic age" of the pulsar.

\subsubsection{Millisecond pulsars}

\section{Distinct population}

Millisecond pulsars (MSPs), those pulsars with spin periods less than $\sim 30 \mathrm{~ms}$, spin much faster and live much longer than the more common "normal" (i.e. 1-sec) pulsars, and they are thought to have been produced by a more complex evolutionary process. MSPs originate from the interaction between normal (or most likely, longdead) pulsars and their binary companions. This interaction leads to a transfer of mass and angular momentum which "spins up" (i.e. increases the rotation speed of) the pulsar to many hundreds of rotations per second. In the non-interacting case the luminosity of a pulsar decays as rotational energy is lost, and eventually the pulsar becomes unobservable. However, if the spin-up process takes place after a pulsar has "died", it will rejuvenate the pulsar and cause the pulsar to become a radio emitter once again. The spin-up process is thus often referred to as "recycling".

The recycling process not only changes the spin period of the pulsar, but also its magnetic field strength, and correspondingly, its spin period derivative $(\dot{P})$ and subsequent evolution. The period derivatives of MSPs are smaller by four to five 
orders of magnitude than those of normal pulsars, and given the much more rapid spin rates, implies that MSPs live far longer than normal pulsars. The surface magnetic fields of the MSPs are approximately four orders of magnitude smaller than those of most pulsars due to unknown mechanisms during the spin-up process (probably related to field burial by the accreted ionized gas).

\section{Properties of MSP emission}

Many studies have shown that the radio emission properties of MSPs and normal pulsars are similar (e.g. Kramer et al. 1999). A comparison of the radio flux densities $\left(S_{\nu}\right)$, where $\nu$ is the observing frequency, and the spectral indices $(\alpha)$ between MSPs and normal pulsars indicates that their emissions are not notably different. The pulsar radio flux density can typically be described by a single power law, $S_{\nu} \propto \nu^{\alpha}$, and from a recent study (Bates et al. 2013) the mean spectral index is -1.4. However, MSP spectral indices may be slightly steeper than those of the normal pulsars (Kramer et al. 1998).

The origin of the radio emission however could be substantially different between MSPs and normal pulsars. The basic radio emission process comes from accelerated charged particles (electrons and positrons) moving relativistically along open but curved magnetic field lines and generating emission and other particles via a cascade process. The charged particles emit radio photons coherently, and as such, the emission is highly non-thermal. The details of these coherent processes are not well understood.

The location of the radio beam for normal pulsars is likely above the surface

near the magnetic poles whereas the origin of the MSP radio emission is in the outer magnetosphere, likely near the outermost closed magnetic field lines. Fermi 
has shown definitively that MSP radio beams are significantly larger than those of normal pulsars, resulting in pulsations from each MSP over nearly a full $4 \pi$ steradians. This large beaming fraction supports the idea that MSP radio beams originate in the outer magnetosphere, in contrast to those from normal pulsars, which would be much narrower and lighthouse-like, from deeper in the dipolar field near the poles.

For the energetic pulsars (i.e. pulsars detected in gamma-rays) several emission models have been proposed. Polar cap models, which assume that gamma-ray photons come from near the surface above the magnetic polar caps, have been disfavored by Fermi LAT (see Section 1.3.1 for more details about the Fermi LAT) observations (Abdo et al. 2010b). The more favored models are the outer-magnetospheric emission models such as outer-gap, slot-gap, two-pole caustic, and pair-starved polar cap models; see Johnson et al. (2014) for a review of these gamma-ray emission models. However, the best-fitted gamma-ray emission models seem to vary from pulsar to pulsar. Fermi has opened a new era in high-energy pulsar studies with a phenomenal 205 gamma-ray pulsars detected in the past 7 years. These discoveries should lead to many additional insights into the complex pulsar emissions processes.

\subsubsection{Pulsar timing}

Pulsar timing monitors neutron star rotations by tracking the arrival times of individual (or averaged) pulses from pulsars. The main point in pulsar timing is that every single rotation is unambiguously accounted for over a long period of time (decades for some pulsars). Due to the clock-like rotational stability of pulsars, the observed rotational phases from pulsations can be precisely tracked. The unambiguity and precision of pulsar timing allows astronomers to make very accurate astrometric and spin measurements of the pulsar, high-precision determinations of orbital parameters, 
and unique measurements of the intervening interstellar medium (ISM). The applications of pulsar timing are various and astonishing, and include testing gravitational theories in the strong field regime, studying the dense interiors of neutron stars, and possibly directly detecting gravitational waves (GWs).

\section{Times of Arrival (TOAs)}

After a new pulsar is discovered, a series of initially densely (but progressively less so) sampled observations are made of the pulsar in order to unambiguously track the rotational phase of the pulsar. Once that phase is established, the pulsar is regularly observed once or twice per month for at least a year to establish a "pulsar timing solution". During these observations, the data receive a time stamp from a reference clock at the telescope (which itself is referenced to GPS). With the time stamp and a stable frequency reference (typically a Hydrogen maser) tracking time during the observation, one can determine an accurate time at any point of the observation.

To create a TOA, the data are "folded" modulo the predicted spin period at the observatory and integrated over many pulses to yield averaged pulse profiles as a function of observing frequency. The dispersed folded pulses in frequency are then corrected for the dispersive interstellar delay (see section 1.1.2 for details on dispersion) and partially or completely integrated over frequency. The resulting pulse profiles are cross-correlated with a noise-free template profile which is based on an averaged pulse profile of high signal-to noise. The cross-correlation measures the time (or phase) difference between the profile and the template. Since the absolute reference time of the data, at the beginning or the middle of the folded integration, is known, the absolute time-of-arrival of the averaged pulse profile can be measured. Fig. 1.4 summarizes the steps in generating TOAs (Lorimer \& Kramer 2005). 


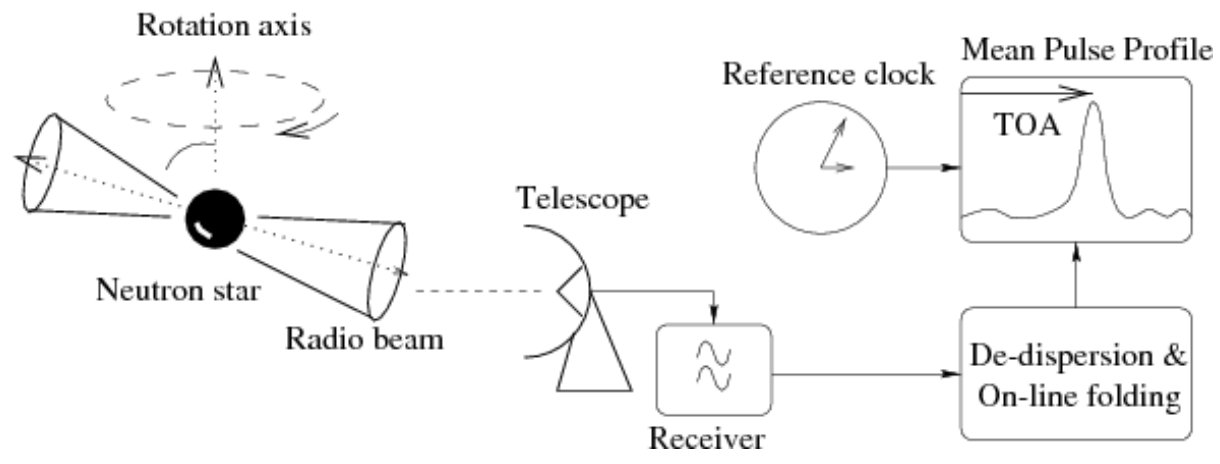

Fig. 1.4.- : Stages of pulsar timing: beginning with observing a pulsar, dedispersion, folding the data to establish an integrated pulse profile, and generating TOAs by cross-correlating the pulse profiles with a template profile. Image credit: Lorimer $\mathscr{E}$ Kramer (2005)

The uncertainty of the TOA $\left(\sigma_{\mathrm{TOA}}\right)$ is roughly proportional to the ratio of the pulse profile's width $(W)$ and its signal-to-noise $(S / N)$,

$$
\sigma_{\mathrm{TOA}} \simeq \frac{W}{S / N}
$$

High $S / N$ MSPs with narrower pulse profiles are therefore preferable for high-precision pulsar timing than normal pulsars. Moreover, the old MSPs are more stable rotators and show much less intrinsic timing noise than young pulsars.

\section{Timing models and timing residuals}

To a good approximation, the Solar System center-of-mass (or barycenter, SSB) is an inertial frame where time advances as a constant rate. In the SSB frame, we can predict the arrival times of pulses observed via TOAs with a simple Taylor expansion of the time-dependent phase of a pulsar, $\phi(t)$, where

$$
\phi(t)=\phi_{0}+f\left(t-t_{0}\right)+\frac{1}{2} \dot{f}\left(t-t_{0}\right)^{2}+\ldots
$$


$\phi_{0}$ and $t_{0}$ are the reference phase and time, and $f$ and $\dot{f}$ are the pulsar's spin frequency and frequency derivative (i.e. spin-down, due to losses of energy to particle and electromagnetic radiation).

Since a telescope is in the frame of a rotating Earth orbiting the Sun, the observed (topocentric) TOAs need to be transformed into the SSB (barycentric) frame. To transform the topocentric TOAs $\left(t_{\text {topo }}\right)$ to barycentric TOAs $(t)$, many time corrections needed to be applied,

$$
t=t_{\mathrm{topo}}-t_{0}+\Delta t_{\mathrm{clock}}-\Delta t_{\mathrm{DM}}+\Delta t_{\mathrm{R}}+\Delta t_{\mathrm{rel}}
$$

where $t_{0}$ is the reference time, $\Delta t_{\text {clock }}$ corrects the observatory clock to an international atomic time standard, $\Delta t_{\mathrm{DM}}$ is the time delay from the dispersion, $\Delta t_{\mathrm{R}}$ is the Römer time delay (projected light travel time from the telescope to the SSB) and $\Delta t_{\text {rel }}$ comprises the Einstein and Shapiro time delays due to general relativistic propagation or clock rate corrections in the Solar System.

We use the program TEMPO to barycenter the TOAs and create a timing model which fits the TOAs via least-squares. The model fitting is performed in an iterative manner by starting the fit with initial pulsar parameters (like the spin period and sky location during the time of discovery) and improving those parameters, and adding others as necessary, as additional observed TOAs and longer timing baselines are accumulated.

The timing residuals (i.e. the difference between the observed TOAs and the timing model) of the best-fit model should optimally show a Gaussian distribution around zero (i.e. flat, white-noise residuals) if the model is appropriate. Whereas errors in timing model parameters cause systematic signatures in timing residuals. Fig. 1.5 shows how timing residuals can be affected by various timing parameter errors. 

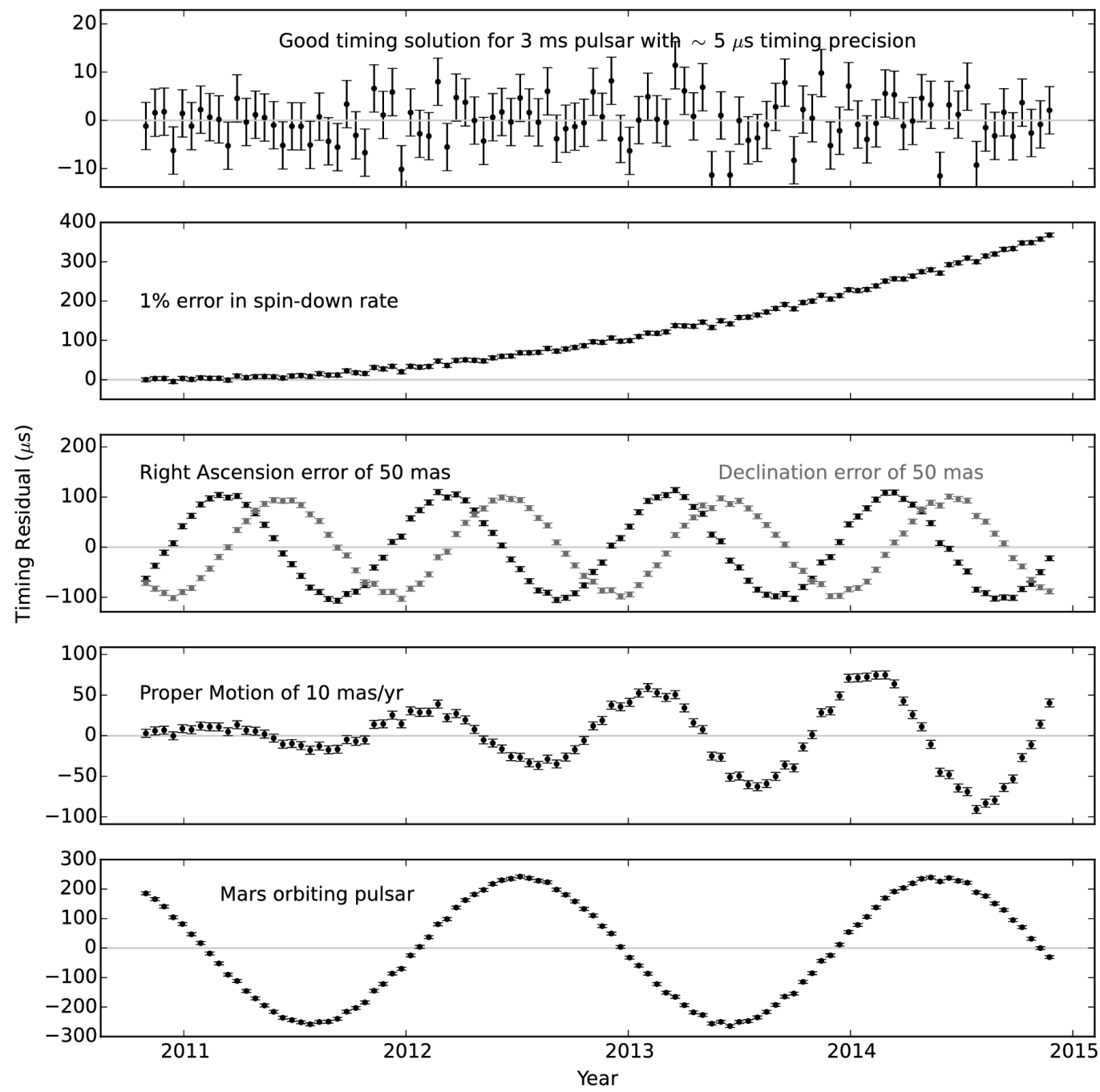

Fig. 1.5.- : Examples of five different sets of pulsar timing residuals. From top to bottom, "good" timing parameters (showing flat residuals), an error of $1 \%$ in spindown rate (showing a quadratic drift in pulse phase), errors in positions by 50 mas (resulting in annual sinusoids), an error of 10 mas/yr in proper motion (showing an annual sinusoid which grows with time), and the presence of a Mars-like planet around the pulsar. Image credit: Scott Ransom 


\section{Timing binary pulsars}

To time binary pulsars, the timing model needs to incorporate additional parameters to compensate for orbital motion. That motion can typically be described using five "Keplerian parameters", including the orbital period $\left(P_{\mathrm{b}}\right)$, projected semi-major axis of the elliptical orbit $(x \equiv a \sin i / c)$, orbital eccentricity $(e)$, longitude of periastron $(\omega)$ and the epoch of periastron passage $\left(T_{0}\right)$.

When $x$ and $P_{\mathrm{b}}$ are measured from pulsar timing, the mass function $\left(f_{\mathrm{m}}\right)$ of the pulsar mass $\left(m_{\mathrm{p}}\right)$, companion mass $\left(m_{\mathrm{c}}\right)$, and orbital inclination $(i)$ can be obtained,

$$
f_{\mathrm{m}}=\frac{4 \pi^{2}}{G} \frac{x^{3}}{P_{\mathrm{b}}^{2}}=\frac{\left(m_{\mathrm{c}} \sin i\right)^{3}}{\left(m_{\mathrm{p}}+m_{\mathrm{c}}\right)^{2}}
$$

where $G$ is the Newton's gravitational constant. In practice, the orbital inclination is unknown, therefore a lower limit of the companion mass can be estimated by assuming $i=90^{\circ}$ and $m_{\mathrm{p}}=1.35 M_{\odot}$.

For the pulsars in compact binary orbits which are more likely to be relativistic, an additional set of "post-Keplerian" (PK) parameters are possibly required in order to achieve high-precision timing solutions. Potentially observable PK parameters include the relativistic advance of periastron $(\dot{\omega})$, a combination of time dilation and gravitational redshift $(\gamma)$, the rate of orbital decay due to gravitational radiation $\left(\dot{P}_{\mathrm{b}}\right)$, and the two Shapiro delay parameters $r$ and $s$. In general relativity, all five of the PK parameters are functions of only the well-measured Keplerian orbital parameters and $m_{\mathrm{p}}$ and $m_{\mathrm{c}}$. By measuring some or all of the PK parameters, one can measure the masses of the pulsar and companion star and potentially test general relativity or other gravitational theories. Since the majority of MSPs are in binaries and can be timed more precisely than normal pulsars, MSPs are ideal for exploring exotic physics 
via pulsar timing.

The Fermi LAT has assisted in the discovery of many rare types of binary MSPs in compact (orbital period of $<1$ day) orbits with low-mass companions $\left(\lesssim 0.3 M_{\odot}\right)$ and which typically show eclipses of the radio pulsations. These so-called "spider" systems are known as "black widows" (if the companions are very low mass, $\lesssim 0.08 M_{\odot}$ ) or "redbacks" (strongly eclipsing binary pulsars with low-mass main sequence companions). Black widows and redbacks were traditionally found in globular clusters where stars are densely packed and stellar interactions can exchange in new companions to the MSPs. The new Fermi identified Galactic black widows and redbacks are therefore fascinating systems to study since they may have different evolutionary origins than those found in globular clusters. They seem to be the "missing links" in pulsar evolution (e.g. Archibald et al. 2009; Papitto et al. 2013) from low-mass x-ray binaries (LMXBs) to MSPs.

Eclipsing pulsars in tight orbits however, are more challenging to time. In some systems the eclipses last more than half of the orbit and ionized gas from the companion star additionally delays the radio pulses. As a result, the TOAs from these systems can be of poor quality. Classical effects from the bloated companion stars randomly perturb the orbits on month and year timescales as well, potentially causing pulse rotational ambiguities if the observation cadence is not dense enough.

\section{Pulsar timing applications}

As mentioned earlier, pulsar timing is a powerful tool which allows astronomers to measure parameters of the pulsar, its possible orbits, and the ISM very accurately. There are various applications from the precisely measured parameters namely:

- By measuring variations in the DM from the pulsar, one can probe the properties 
of the ISM between the pulsar and the observer.

- For binary pulsars in eccentric orbits, like the first binary pulsar B1913+16, the PK parameters $\dot{\omega}$ and $\gamma$ can be measured precisely. That allows astronomers to accurately determine the mass of pulsar and the companion. For B1913+16, $\dot{P}_{\mathrm{b}}$ was eventually detected, which implied the existence of the orbital decay due to the emission of gravitational radiation as predicted by GR (Weisberg \& Taylor 2005).

- For the double pulsar system, J0737-3039, all five PK parameters have been precisely measured. These measurements show that GR is correct to better than $0.05 \%$ and precisely provided the masses of both pulsars with fractional uncertainties of $10^{-4}$ (Kramer et al. 2006).

- The detection of the general relativistic Shapiro delay from pulsar timing of the binary pulsar PSR J1614-2230 allowed us to infer the mass of both the pulsar and the companion very precisely. The pulsar mass is $1.97 \pm 0.04$ which was the heaviest high-precision pulsar mass known to date (Demorest et al. 2010). Its measurement has provided a very strong constraint on the physics of matter at supra-nuclear densities, and in particular, the so-called neutron star Equation of State (EOS).

Over the last decade, the direct detection of gravitational waves (GWs), the distortions of space-time caused by the motions of exotic and massive compact objects, has become a major goal of pulsar studies. By timing arrays of MSPs distributed over the whole sky for many years, so-called pulsar timing arrays (PTAs) are looking for correlated distortions in the timing residuals from nanoHertz frequency GWs passing through our galaxy. The sources of the GWs are likely to be supermassive black hole 
binaries scattered throughout the universe. The International Pulsar Timing Array (IPTA) is the collaboration of three PTA organizations: NANOGrav in North America, and the Parkes (in Australia) and European PTAs. Given the improvement in pulsar timing from the IPTA, the GWs could possibly be detected within five to ten years. A crucial improvement in GW sensitivity comes from adding new MSPs to PTAs and the recent search successes, especially aided by Fermi have dramatically contributed to PTA science.

\subsection{Searching for New Pulsars}

The periodic signals from the first pulsar, PSR B1919+21, were discovered in 1967 by visually inspecting the total power output from a radio telescope in Cambridge, England and directly seeing individual pulses. However, the majority of known pulsars to date are much too weak to be found by searching for individual pulses and therefore require more sophisticated methods in order to search for their faint pulsations. Currently, "standard" radio pulsar search procedures are performed in both the time and frequency domains via de-dispersion of channelized data and then Fourier analysis of the resulting time series. Additionally, more advanced techniques, namely acceleration searches and single-pulse searches, increase our sensitivity to exotic pulsars in binary systems as well as rare but bright giant pulses from certain pulsars, respectively.

The standard procedure used in searching for the unknown spin period and dispersion measure (DM), an integral of the a priori unknown free electron number density along the line of sight between a pulsar and an observer, is briefly summarized as follows. The data are de-dispersed and integrated over observing frequency at a wide range of trial DMs resulting in a number of time series. Each time series is Fourier 
transformed, then typically squared to make a power spectrum, and then possible harmonic information is summed in various combinations to near-optimally recover all the power from pulsations. The results of the periodic search process are saved and are then human-inspected and/or processed by machine learning programs in order to find good pulsar candidates. For the best candidates, the raw data folded at the fundamental frequency found in the Fourier analysis in order to further investigate whether the candidates are real pulsars. The processes is repeated for each trial DM.

\subsubsection{RFI removal}

Radio Frequency Interference (RFI) is interference from terrestrial (or satellite) radio transmitters that can significantly contaminate the data and reduce our sensitivity to detect new pulsars. Some RFI imitates the signature produced by periodic signals from pulsars. If RFI is not treated properly, it can overpower the pulsar signals, causing them to be only weakly detected or even missed all together. RFI must therefore be carefully removed before starting the pulsar searches.

The potential sources RFI are many, from electrical storms, to nearby electrical devices (microwaves, laptops etc.), and much further transmitters such as radars, aircraft, and satellites. The worst RFI for pulsar searching are those with pulsing broadband signals which are therefore similar to the periodic signals from pulsars. Fortunately, these sources are terrestrial and therefore are not dispersed in the same manner as those from pulsars which traveled through the ionized ISM. The majority of RFI instances have apparent DMs of zero. We attempt to remove interference at several different stages of the search pipeline, from initial data processing and dedispersion, to "zapping" of known periodic signals from the Fourier power spectra, to post-facto discarding of folded candidates with non-pulsar-like characteristics. 


\subsubsection{Dispersion Measure trials}

As the DM towards a pulsar is unknown prior to discovery, many DM trials must be searched. To save time and computer power, the step sizes over DM must be optimized. The steps must not be so large that the true DM value falls well between two trial DMs, nor so small that computations are wasted. Determining an ideal DM step size is especially crucial when it comes to detecting pulsars with short spin periods, because the $\mathrm{S} / \mathrm{N}$ of a detection reduces strongly as the error in DM increases, in a manner proportional to the spin frequency of the pulsar. The optimal DM step size also depends on many observing factors, namely the full range of trial DMs, the central observing frequency, the observing bandwidth, and the sample time.

We used DDplan . py from PRESTO to determine the DM step sizes for each observation. The optimal DM range for searching can be estimated from the maximum DM value from a model of the electron density distribution in the galaxy (e.g. NE2001 (Cordes \& Lazio 2002)) multiplied by a factor of two (to account for the uncertainty in the model). In general, a maximum DM of more than $1000 \mathrm{~cm}^{-3} p c$ is expected for surveys along the Galactic Plane and less than $\sim 50 \mathrm{~cm}^{-3} p c$ for high-galactic-latitude surveys. After determining the DM step size and range, we incoherently de-disperse the raw search data into time series at each trial DM value, by shifting the arrival times of each frequency channel according to the DM and summing across the observing band.

\subsubsection{Periodic Searches}

The most widely used technique for periodicity searching is to Fourier transform the de-dispersed time series and examine them in the frequency domain. Since the time series are formed from independently, and typically uniformly, sampled data points, 
we use the Fast Fourier Transform and then convert the Fourier amplitudes to powers to make a power spectrum.

The periodic signals from pulsars typically have small duty cycles (the pulse width divided by the spin period). As a result, the signals appear in the Fourier domain as numerous evenly-spaced narrow peaks, comprising the fundamental frequency and a number of harmonics (each separated by the spin frequency of the pulsar). To increase the detection significance for pulsars with such narrow pulses, the fundamental frequency power and up to 32 harmonics are summed together. This technique is called "harmonic summing". The smaller the duty cycle, the more harmonics are able to be summed to reach the optimal gain. The best fundamental frequency as determined from harmonic summing (and which includes Fourier interpolation) is then converted to a best spin period in the time domain. The best periods from each time series are saved for further investigation.

\subsubsection{Acceleration Search}

For pulsars in binary systems, the binary motion causes a slight change in the observed spin period due to the Doppler effect. This results in a distribution of pulsation power over multiple Fourier bins in the frequency domain, which dramatically reduces the search sensitivity. To mitigate this effect, we performed "acceleration searches".

The Doppler equation of an observed pulsar frequency as a function of time, $\nu(t)$ $=1 / \operatorname{spin}$ period, is

$$
\begin{gathered}
\nu(t)=\nu_{0}\left(1-\frac{V_{l}(t)}{c}\right) \\
V_{l}(t)=a_{l} t+V_{l}(0),
\end{gathered}
$$

where $\nu_{0}$ is the intrinsic frequency, $V_{l}(t)$ is the line of sight velocity of a pulsar, $c$ is the 
speed of light and $a_{l}$ is the line of sight acceleration of a pulsar. The acceleration is simply assumed to be constant during the observation, and is an additional parameter to search for during the periodic searching process.

We used PRESTO's routine accelsearch to account for the signal drifting over Fourier bins due to orbital acceleration and to perform a periodic search using Fourier interpolation and harmonic summing of 1, 2, 4, 8 or 16 harmonics. The acceleration search is a crucial part of the full search process here as we expect most MSPs to be in binary systems.

\subsubsection{Candidate selection}

For each observation, the result after RFI removal, de-dispersion, and acceleration searching are candidate periodic signals with a spin period and a DM value. The time series are then folded at the spin period and either visually inspected or passed to machine learning software to find pulsar-like signals. After some good candidates are found in the time series, the raw data are folded (a much more time-consuming process) to see if the candidate's peak at non-zero DMs and otherwise appear to be real pulsars. The key features of a real pulsar on a candidate plot are a continuous signal (straight line) in both time-phase and frequency-phase plots and a sharp peak (at non-zero DM) on the DM plot. Fig. 1.6 shows a plot of the de-dispersed and folded raw data of the pulsar, PSR J2042+0249.

\subsubsection{Single-pulse search}

Besides periodic pulses, pulsar emission may occasionally vary greatly in amplitude and result in apparent sporadic signals. Some pulsars, for example, exhibit nulling behaviour which means that the pulsations "turn off" and then "turn on" at some 

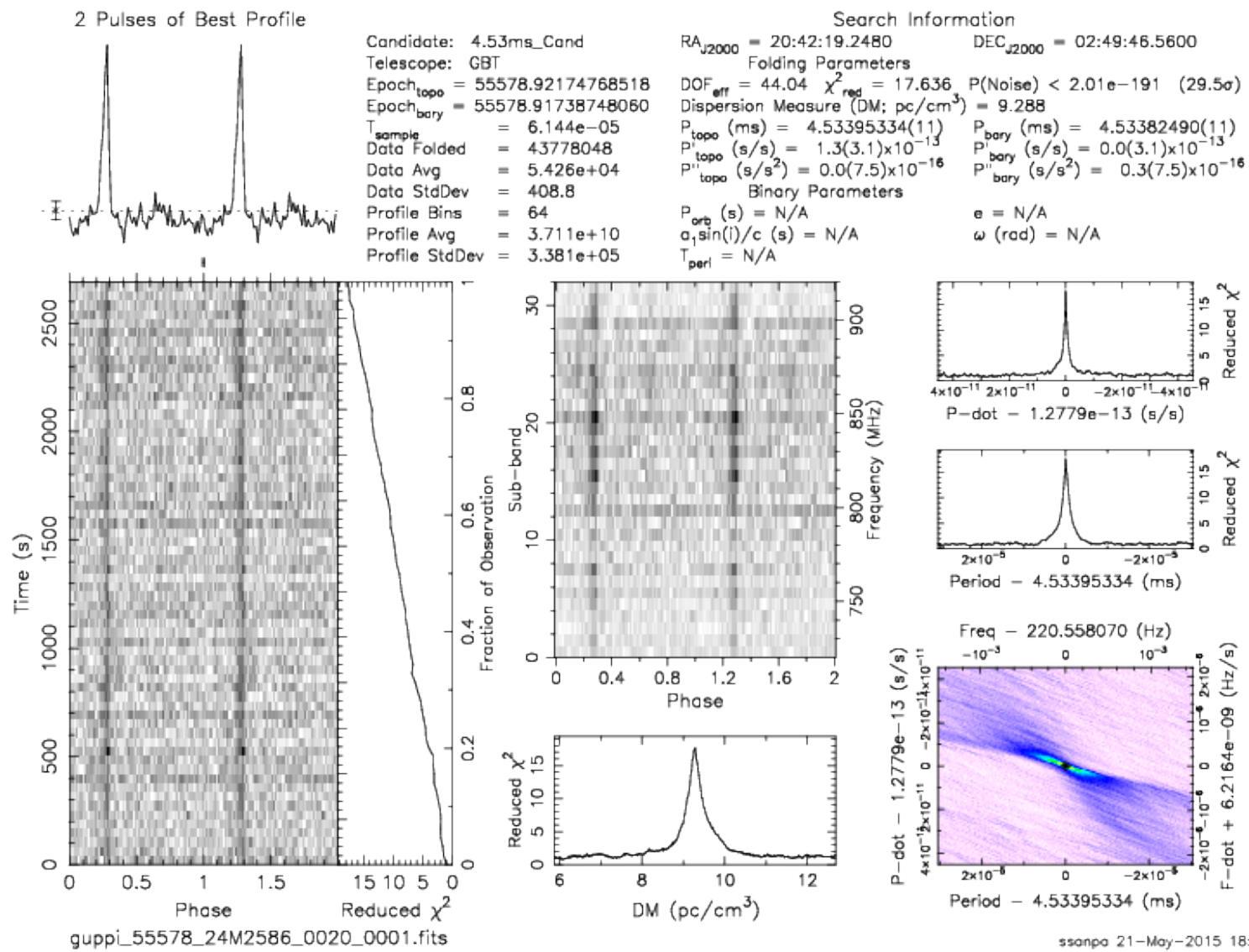

Search Information

$53 \mathrm{~ms} \_$Cand

elescope: GBT

Folding Porameters

poch $=55578.91738748060$

$=6.144 \mathrm{e}-05$

$=43778048$

$=5.426 \mathrm{e}+04$

$=408.8$

$=64$

$=3.711 \mathrm{e}+10$

$T=N / A$

$\omega(\mathrm{rod})=N / A$
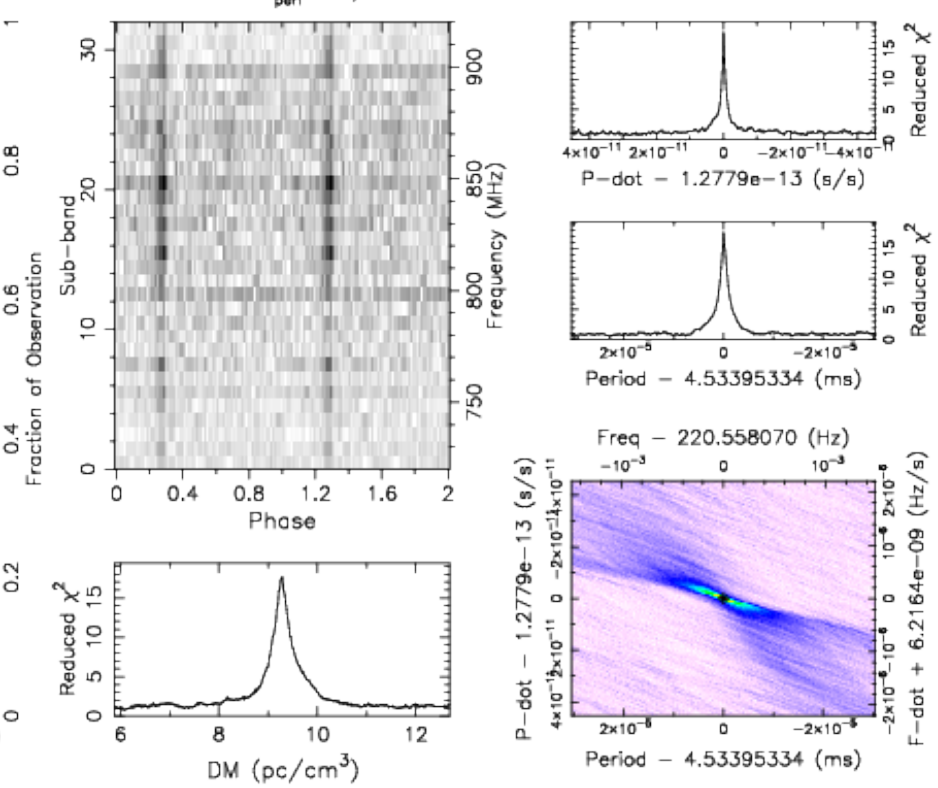

Period - 4.53395334 (ms)

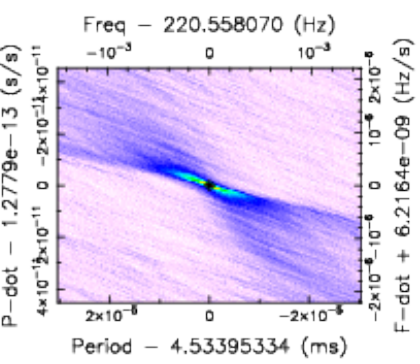

ssonpo 21-Moy-2015 18:38

Fig. 1.6. - : This is a raw-data-folded plot of PSR J2042+0249. It shows all the features of true pulsar signals. 
later time interval. Other examples include the rotating radio transients (RRATs) (McLaughlin et al. 2006) which are thought to be old rotating neutron stars which only rarely emit a pulse of radio emission. The periodicity search in the Fourier domain is not sensitive to this type of radiation from pulsars; therefore the "single-pulse search" technique has to be applied.

The concept of single-pulse searches is simple. Instead of searching for periodic signals for each trial DM, each time series is examined for large individual pulses. If a pulsar is a sporadic emitter, or emits giant pulses, those events can be detected in a correctly de-dispersed time series using simple matched-filtering with a boxcar signal.

Searching for single pulses in time series is essentially finding events in the time series which deviate from the mean by several standard deviations (given that the time series has Gaussian noise with known mean and standard deviation). We used PRESTO's single_pulse_search.py to search for single pulses in the time series. Fig. 1.7 shows a result from the single-pulse searches.

\section{$1.3 \quad$ Fermi Unassociated Sources}

\subsubsection{Fermi satellite}

The Fermi Gamma-ray Space Telescope was launched in 2008 with two main instruments on board: the Gamma-ray Burst Monitor (GBM) and the Large Area Telescope (LAT). This thesis focuses on sources detected with the LAT. The LAT's field of view is about $2.4 \mathrm{sr}$, and the main operational mode is a sky survey mode which covers the entire sky every three hours. The LAT detects gamma-ray photons with energies ranging from $20 \mathrm{MeV}$ up to over $300 \mathrm{GeV}$, and is the most sensitive gamma-ray telescope to date. 

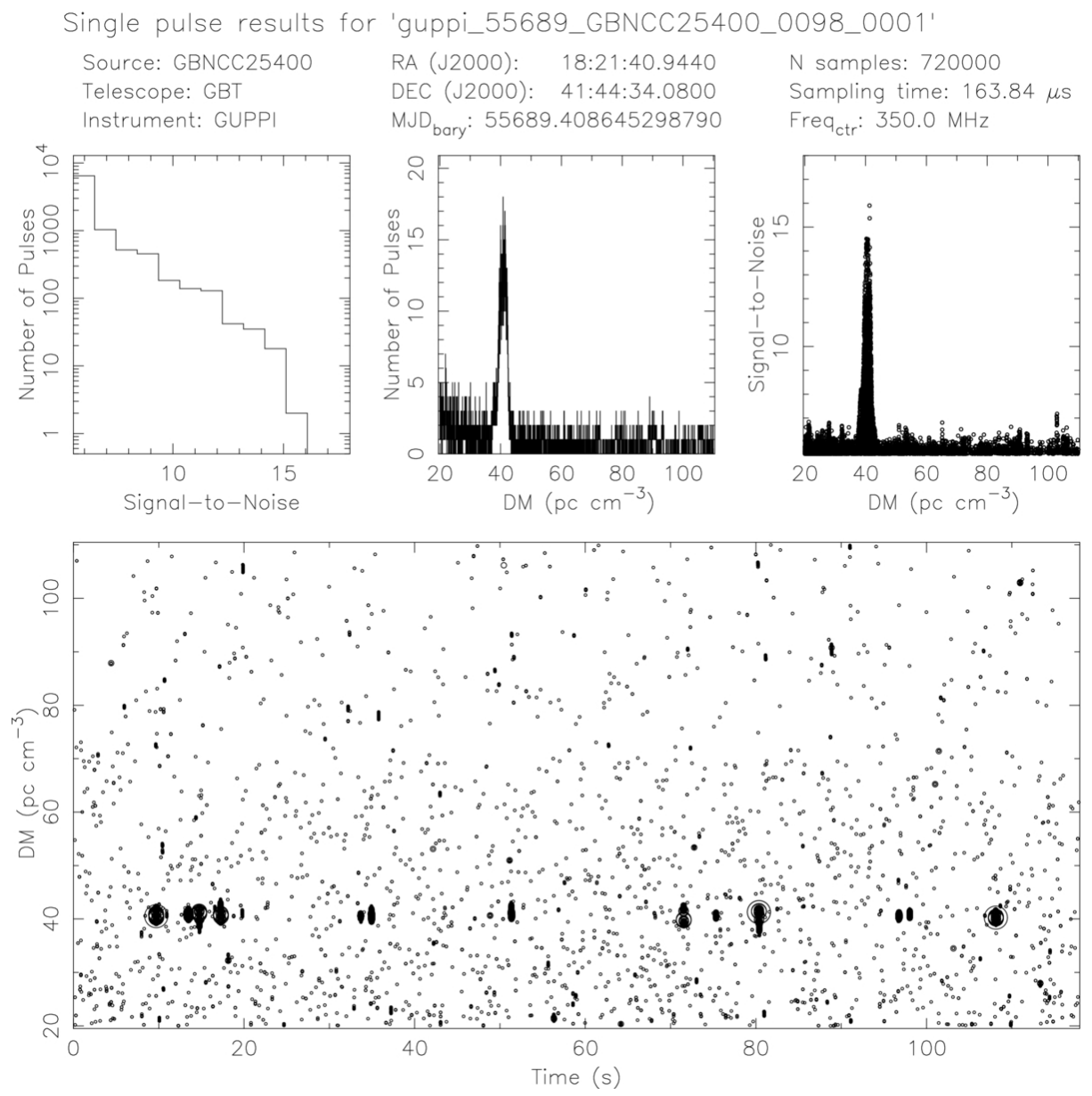

Fig. 1.7.- : Single-pulse search results for pulsar J1821+41. The top left panel shows $\mathrm{S} / \mathrm{N}$ versus number of detected pulses. The middle and right panels show DM versus number of pulses and DM versus $\mathrm{S} / \mathrm{N}$, respectively, which both peak at DM of $\sim 40 \mathrm{pc} \mathrm{cm}^{-1}$. The bottom panel shows integration time versus DM: the darker the dots (or the bigger the circles), the higher the $\mathrm{S} / \mathrm{N}$ of the pulses detected. All panels suggest that this pulsar emits single pulses sparsely at a DM of $\sim 40 \mathrm{pc} \mathrm{cm}^{-1}$. 
In this thesis I used data from all three Fermi source catalogs, 1FGL (Abdo et al. 2010a), 2FGL (Nolan et al. 2012) and 3FGL (Acero et al. 2015), which are based on 11 months, 2 years and 4 years of LAT data, respectively.

\subsubsection{Fermi "Treasure Map"}

After years of continuously mapping the gamma-ray sky, the LAT has revealed thousands of gamma-ray sources. The third Fermi LAT source catalog (3FGL), for example, includes 3033 gamma-ray sources above $4 \sigma$ significance, and 1010 of them are unassociated with other astrophysical sources.

There are three techniques typically used to determine whether any of these unassociated sources are pulsars. The first and most straight forward technique is to temporally fold the gamma-ray data with known pulsar ephemerides if the source position is consistent with a known pulsar location; this technique has revealed 6 gamma-ray pulsars (Abdo et al. 2009a). The second technique is to blindly search for pulsations in the LAT data. Though this is a very algorithmically and computationally difficult task, it has resulted in the discovery of over 37 new gamma-ray pulsars (e.g. Abdo et al. 2009a; Saz Parkinson et al. 2010; Pletsch et al. 2012a,b)

The last and the most promising technique is to observe the unassociated sources in the radio, and search for radio pulsations. This technique was used on unidentified sources found by the previous generation gamma-ray telescope, Energetic Gamma Ray Experiment Telescope (EGRET), where it was unsuccessful in finding any new gamma-ray pulsars (Thompson 2008). The main reason for the poor success in the past was EGRET's large positional uncertainty of typically several degrees. The lack of a precise location necessitates multiple telescope pointings to cover the whole error box, and therefore makes deep radio searches on each source very inefficient. 
The localizations for the LAT unassociated sources are much better than those from EGRET, typically 10-30 arcmin in size, and so most can be covered by a single pointing with a radio telescope like the GBT. Our target lists for the pulsar searches use gamma-ray criteria laid out by the Fermi team: namely, that they be unassociated sources with exponential spectral cutoffs and low variability (see Fig. 1.8 and 1.9).

\subsubsection{The Pulsar Search Consortium (PSC)}

The Pulsar Search Consortium (PSC) is an international collaborators of radio astronomers from all over the world (Ray et al. 2012). The goal is to search for new pulsars in the Fermi LAT unassociated sources and to perform follow-up observations on any new pulsars.

The Fermi LAT and the PSC have provided a breakthrough in the pulsar search community by discovering 70 new millisecond pulsars among the Fermi unassociated sources (e.g. Ransom et al. 2011; Keith et al. 2011; Bhattacharyya et al. 2013; Camilo et al. 2015). One of the interesting results from the Fermi LAT is that 70 out of 72 new pulsars are MSPs in the Galactic disk (those outside globular clusters), and 54 of them are already confirmed as gamma-ray emitters (the remaining ones will likely be proven so with longer timing). Given that it took over 27 years to find 60 Galactic MSPs prior to the the launch of Fermi, the discovery of 70 Galactic MSPs in 8 years is fascinating. In addition to the successful rate of discovery, among these 70 new MSPs at least 28 of them are black widows or redbacks, previously rare and exotic interacting pulsar binary systems. Only 3 black widows and 1 redback were known in the Galactic plane at the time of the Fermi launch. 


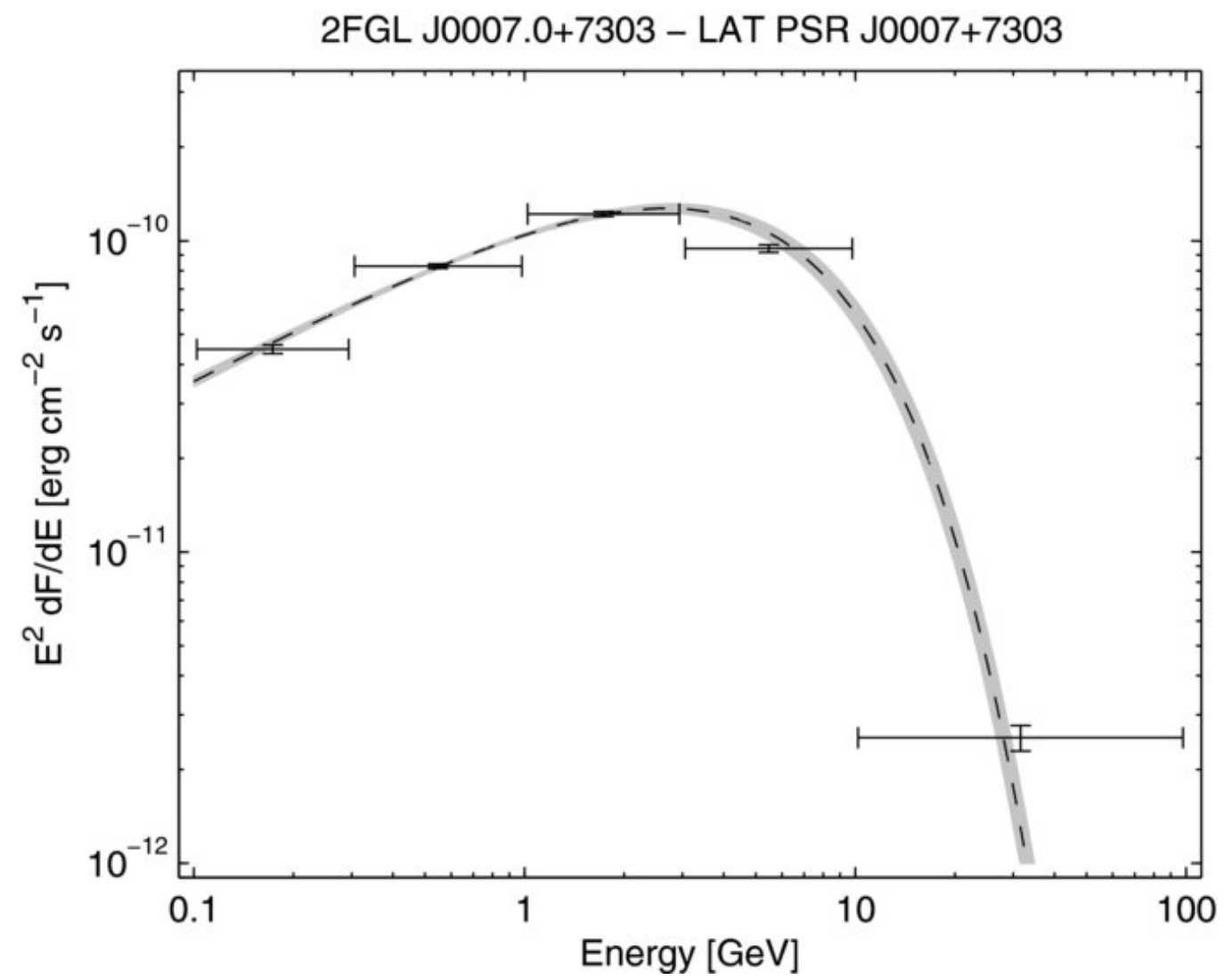

Fig. 1.8.- : An example plot of a gamma-ray spectrum with an exponential cutoff within a few GeV range (Abdo et al. 2013). This spectral shape is one of the characteristics of gamma-ray pulsars. 


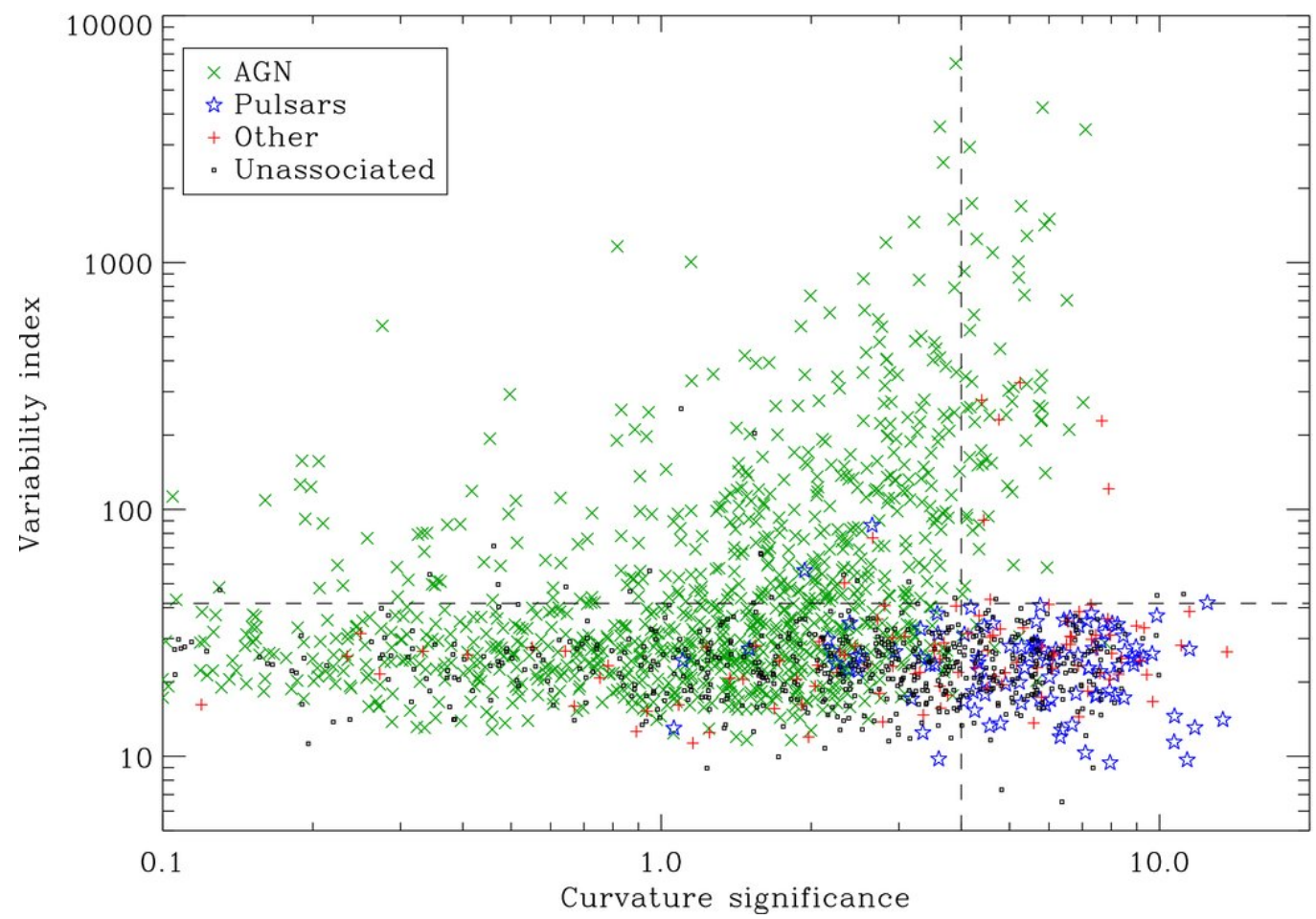

Fig. 1.9.- : Variability and Curvature (i.e. the probability of having a cutoff spectra) statistics of 2FGL sources (Abdo et al. 2013). The known pulsars (blue stars) fall into the region of low variability and high curvature. Therefore, the unassociated sources which fall into the same region are likely to be pulsars as well. 


\subsection{Pulsar Search with the Green Bank Telescope (GBT)}

The Robert C. Byrd Green Bank Telescope (GBT) is the world largest fully steerable single-dish radio telescope. The GBT's 100-meter diameter dish, unblocked aperture and outstanding surface accuracy provide excellent sensitivity across the telescope operation wavelength range from 0.1 to $116 \mathrm{GHz}(3.0 \mathrm{~m}$ to $2.6 \mathrm{~mm})$. It is also located in the National Radio Quiet Zone, where radio transmitters are under control by the government in order to provide the most "quiet" radio environment for the GBT.

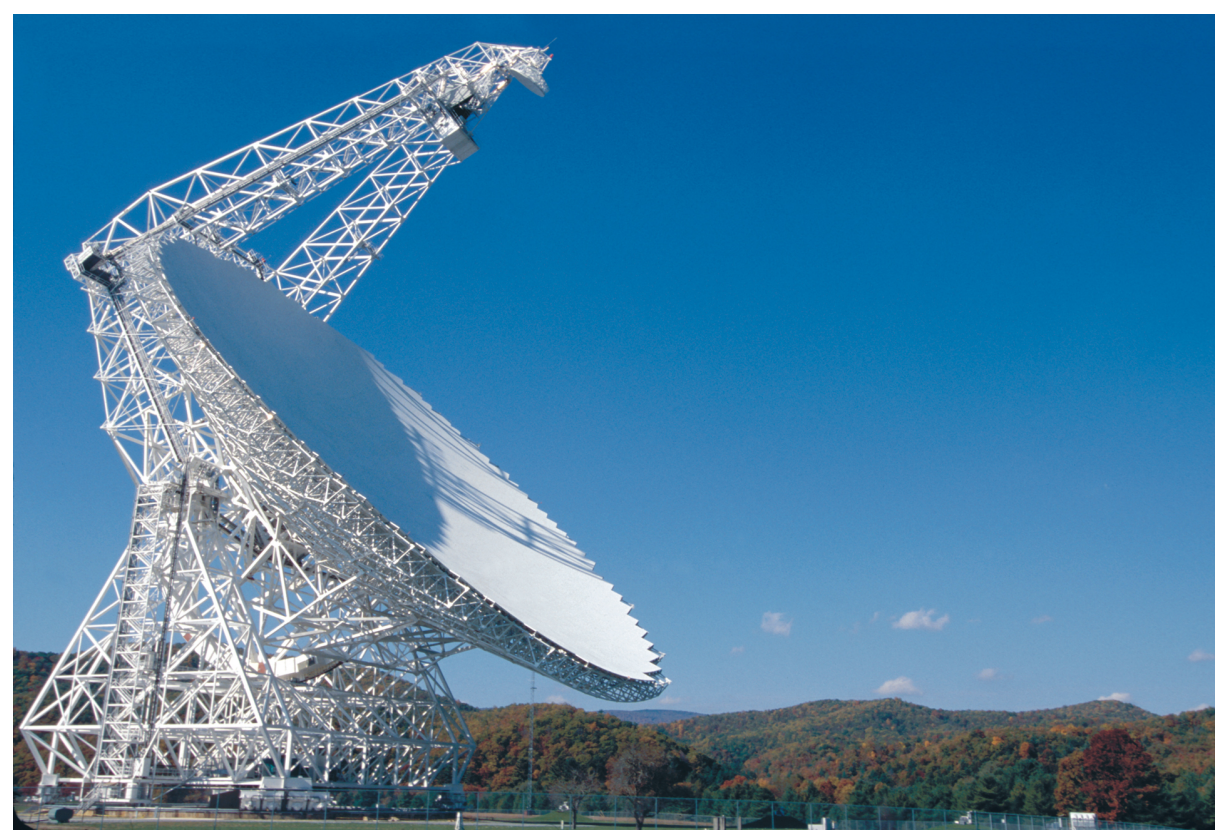

Fig. 1.10.-: The Robert C. Byrd Green Bank Telescope (GBT). Image creit: NRAO

The GBT is one of most successful telescopes for pulsar searches as a result of its excellent sensitivity and the Green Bank Ultimate Pulsar Processing Instrument (GUPPI), which is the backend designed specifically for high-performance and wideband pulsar observations. The GBT has found over 250 new pulsars through largearea surveys, such as the GBT $350 \mathrm{MHz}$ Drift-scan survey and the Green Bank North 
Celestial Cap survey (GBNCC) (e.g. Lynch \& Bank North Celestial Cap Survey Collaborations 2013), and deep observations of special targets like supernova remnants, globular clusters (e.g. Ransom et al. 2005), and now, Fermi unassociated sources.

For the targeted pulsar searches of the Fermi unassociated sources, the GBT has played a very major role, discovering 34 of the 70 new MSPs uncovered by the PSC. The discovery of twelve new MSPs with the GBT is discussed in Chapter 2.

For this thesis, we observed more than 100 Fermi unassociated sources with the GBT at three different observing frequencies, and are reporting the discovery and timing solutions of 16 of them, including 4 rare "spider" pulsars. The author discovered 10 of these pulsars herself, conducting large-scale acceleration searches on a computer cluster located at NRAO in Charlottesville, VA. 


\section{Chapter 2}

\section{Discovery of Twelve New}

\section{Millisecond Pulsars in Fermi LAT}

Sources with the Green Bank

\section{Telescope}

\section{$2.1 \quad$ Introduction}

After seven years of operation, the Large Area Telescope (LAT) on board the Fermi $\gamma$-ray Space Telescope (Atwood et al. 2009) has revolutionized pulsar astronomy by enabling the discovery of many new radio millisecond pulsars (MSPs). Prior to the launch of Fermi, radio telescopes searched for pulsars in the error boxes of unassociated $\gamma$-ray sources from EGRET (Energetic Gamma Ray Experiment Telescope) on board the Compton Gamma Ray Observatory (CGRO) (e.g. Roberts 2002; Champion et al. 2005; Crawford et al. 2006; Keith et al. 2008). However, the relatively large positional uncertainty of EGRET sources (approximately 1 degree) exceeded the size 
of the typical primary beams of large radio telescopes, thereby requiring multiple pointings to cover the gamma-ray sources. This decreased both the sensitivity of the searches and the number of sources which could be observed, and therefore no new pulsars were found, with the possible exception of MSP J1614-2230 (Crawford et al. 2006).

The Fermi LAT has much better sensitivity and spatial resolution from $100 \mathrm{MeV}$ to $100 \mathrm{GeV}$ compared to EGRET, resulting in more $\gamma$-ray sources in general, and more with source sizes comparable to those of typical radio beams. Single radio pointings can cover the entire error region providing longer observing times and better sensitivity for pulsar searches. To conduct the radio follow-up observations of LAT sources, the Fermi Pulsar Search Consortium (PSC), an international collaboration of LAT members and pulsar experts associated with single dish radio telescopes, was established (Ray et al. 2012). After performing a number of radio observations on non-variable and unassociated sources from the first series of LAT external and internal source catalogs, Bright Source List (BSL), 1FGL, 2FGL and 3FGL (Abdo et al. 2009b, 2010a; Nolan et al. 2012; Acero et al. 2015, respectively), 71 new MSPs have been discovered. Given that it took nearly 25 years to find 75 MSPs in the Galactic disk prior to Fermi, the discovery of over 70 new Galactic MSPs in seven years is phenomenal.

The National Radio Astronomy Observatory's Robert C. Byrd Green Bank Telescope (GBT) has played a key role in these searches by discovering the first three radio MSPs from the LAT unassociated sources (Ransom et al. 2011), and since then an additional 34 MSPs to date. The first new MSPs triggered the global discoveries of 15 MSPs with the Parkes telescope (e.g. Keith et al. 2011; Kerr et al. 2012), 8 MSPs with the Giant Metrewave Radio Telescope (GMRT) (e.g. Bhattacharyya et al. 
2013), 9 MSPs with the Arecibo telescope (e.g. Camilo et al. 2015; Cromartie et al. 2016), 3 MSPs with the Nançay telescope (e.g. Cognard et al. 2011), 1 MSP with the Effelsberg (Barr et al. 2013) and 1 MSP with the Low Frequency Array (LOFAR) (Pleunis et al. in prep.)

This chapter presents 12 new MSPs discovered in Fermi LAT unassociated sources with the Green Bank Telescope (GBT). We also present radio timing, $\gamma$-ray analyses, and single-pulse searches of the pulsars.

\subsection{Source Selection}

The process of selecting targets to search for pulsations has been an ongoing effort as the Fermi mission has continued to collect data. At the same time, the LAT Collaboration has refined their all-sky analysis methods to account for an improved understanding of the Galactic diffuse emission, as well as the discovery of new and/or unexpected components in the analysis. This study has used inputs from both the 1FGL (Abdo et al. 2010a) and 2FGL (Nolan et al. 2012). catalogs, as well as a preliminary 4-year source list provided through the Pulsar Search Consortium memorandum of understanding (Ray et al. 2012). The $\gamma$-ray sources selected for investigation in this program fall into three distinct categories: new unassociated sources, previously searched bright pulsar-like sources, and non-pulsar associated sources with pulsar-like $\gamma$-ray spectra.

With each release of a new catalog from the Fermi-LAT collaboration, a large and increasing number of unassociated $\gamma$-ray sources have been detected: $630,1,171$, and 3,033 from the 1FGL, 2FGL and 3FGL catalogs respectively. These are previously unknown sources whose positions are not strongly associated with a known $\gamma$-ray emitting counterpart (probability of association $<80 \%$ ) when compared against cat- 
alogs of known gamma-ray source classes, and taking local source density of each catalog into consideration. We considered all sources at Galactic latitudes above $|b|$ of $2^{\circ}$. This category makes up the majority of the sources we searched. We prioritized sources with little or no $\gamma$-ray variability and spectra with exponential cut-offs at a few GeV.

A subset of the bright sources had clearly pulsar-like spectra and are almost certainly pulsars. These sources have been searched multiple times by various radio telescopes at several different frequencies. The non-detections may have resulted from unfavorable diffractive scintillation in the ionized interstellar medium (ISM), or from absorption or scattering in so-called black-widow andor redback systems, which have significant amounts of ionized material escaping from their companion stars.

Finally, we searched a small set of non-varying $\gamma$-ray sources that have non-pulsar associations, but that have $\gamma$-ray spectra that appear clearly pulsar-like. The FermiLAT catalog association process invariably includes some false positive associations. In order to select good pulsar candidates from the associated source population, we required first that all sources be non-varying and have significant curvature in their $\gamma$-ray spectra. In addition, sources with significant emission above $10 \mathrm{GeV}$ were eliminated from the list, as the cutoff in $\gamma$-ray pulsar spectra makes such high-energy emission unlikely for all but the most powerful pulsars.

For all categories, we considered only sources visible from the GBT (i.e. with Dec $\left.>-40^{\circ}\right)$. In all, we observed 198 unique Fermi $\gamma$-ray sources. The names of the sources, the positions observed, and the durations and frequencies of the observations are given in Tables 5.6 and 5.7. 


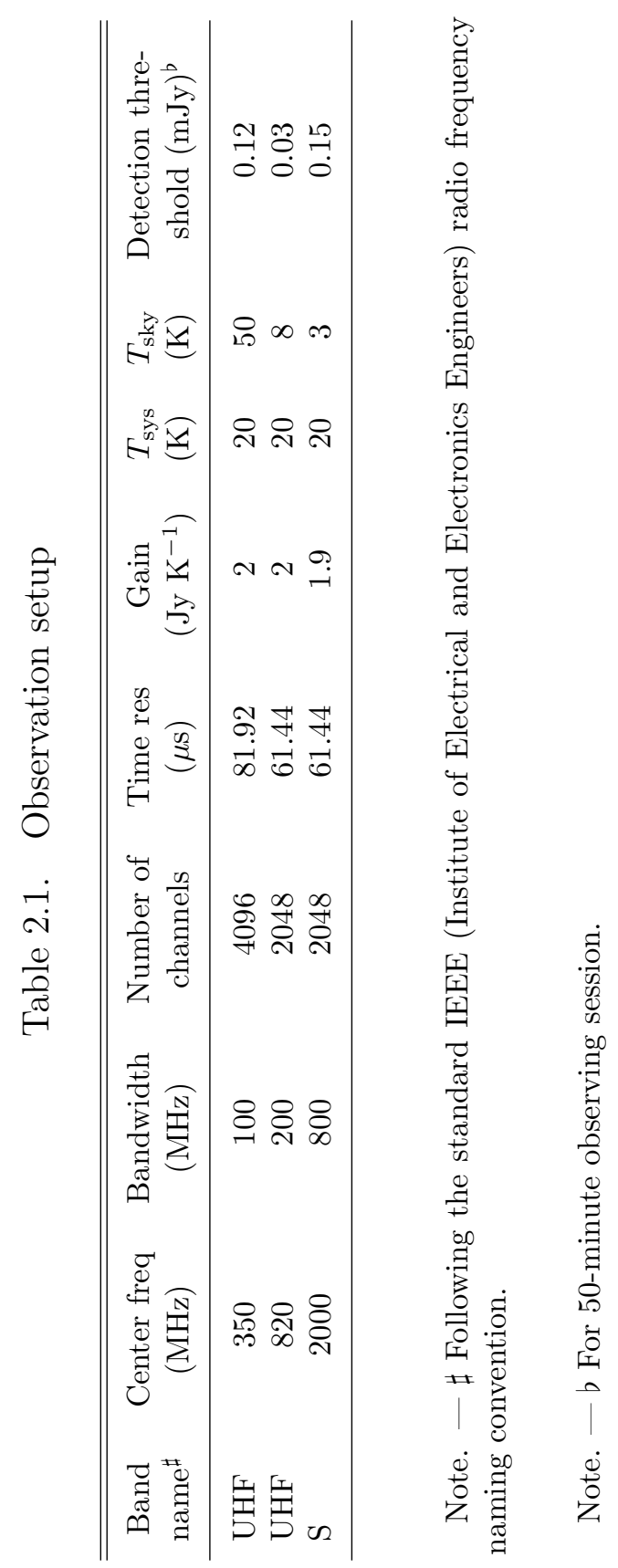




\subsection{Observation and Data Analysis}

\subsubsection{Observation Method and Sensitivity}

According to the selection criteria described in the previous section, we selected 198 sources from 1FGL, 2FGL, internal LAT 3-year, and 4-year source lists. From 2010 December to 2013 April, we observed these sources for approximately 40-50 minutes each, using the prime focus receiver of the GBT centered at either $350 \mathrm{MHz}, 820 \mathrm{MHz}$ or $2 \mathrm{GHz}$ depending on the size of the $95 \%$ Fermi error regions and the Galactic location (particularly latitude) of the sources.

The majority of high Galactic latitude $\left(|b|>2^{\circ}\right)$ sources have error boxes of approximately $13^{\prime}$, thus each source was covered by a single pointing of the GBT at $820 \mathrm{MHz}$ (with a beam FWHM of $16^{\prime}$ ). For sources with error regions larger than $13^{\prime}$ and $|b|>5^{\circ}$, we used the $350-\mathrm{MHz}$ receiver (with a beam FWHM of $36^{\prime}$ ). In order to observe Galactic plane sources $\left(|b|<2^{\circ}\right)$, we used the S-band receiver which is centered at a much higher frequency of $2 \mathrm{GHz}$. Moving to higher frequencies reduces the contribution of the Galactic synchrotron background $\left(\propto f^{-2.6}\right.$, steeper than the typical pulsar flux density spectrum which scales as $\propto f^{-1.41}$ (Bates et al. 2013)). However, the GBT's S-band has a FWHM of only $6.2^{\prime}$, thus, in order to cover the 95\% error regions for most of the low-latitude sources, the observations used multiple pointings (typically 7 ) arranged in a hexagonal grid ( $\sim 10$ minutes per pointing).

For all three observing bands the total intensity signal was recorded with the Green Bank Ultimate Pulsar Processing Instrument (GUPPI) backend in search mode (DuPlain et al. 2008). Bandwidth, number of channels per band and sampling time are given in Table 2.1. The raw data were recorded to hard drives and processed offline. 
To determine the minimum detectable flux density $\left(S_{\min }\right)$ of the observation, we used the radiometer equation (Lorimer \& Kramer 2005)

$$
S_{\text {min }}=\frac{(\mathrm{S} / \mathrm{N})_{\min }\left(T_{\text {sys }}+T_{\text {sky }}\right)}{G \sqrt{n_{\mathrm{p}} t_{\mathrm{obs}} \Delta f}} \sqrt{\frac{P_{\text {cycle }}}{1-P_{\text {cycle }}}}
$$

where the signal to noise ratio threshold $(\mathrm{S} / \mathrm{N})_{\min }=8$; the number of summed polarisations $n_{p}=2$; and expected pulse duty cycle (pulse width over the spin period) $P_{\text {cycle }}=0.1$. Table 2.1 lists the rest of the parameters used: telescope gain $G$, system temperature of the GBT $T_{\text {sys }}$, and sky temperature $T_{\text {sky }}$. The resulting detection thresholds $S_{\min }$ are $0.12,0.03$ and $0.015 \mathrm{mJy}$ for $t_{\mathrm{obs}}=50 \mathrm{~min}$ observations at $350 \mathrm{MHz}, 820 \mathrm{MHz}$ and $2 \mathrm{GHz}$ respectively. The multiple pointing observations yield $S_{\min }$ of $0.03 \mathrm{mJy}$ for a 10 -minute integration time at $2 \mathrm{GHz}$.

\subsubsection{Pulsar Search Method}

\section{RFI Removal}

The data were processed on a 20-node computer cluster at NRAO in Charlottesville, Virginia using standard tools in the PRESTO pulsar software package ${ }^{1}$ (Ransom 2001). Radio frequency interference (RFI) can contaminate pulsar signals, so we searched for both prominent narrow-band and persistent short-duration broadband RFI. We used the routine $r f$ if ind to examine the prominent RFI. For persistent low-level RFI, we searched for periodic signals in a total-power time series de-dispersed at DM of 0.0. Then, we created an RFI mask to replace those "bad" data with channel running median values during de-dispersion. We also removed a "zap list" of known periodic signals from the Fourier power spectra of the de-dispersed time series.

\footnotetext{
${ }^{1}$ http://www.cv.nrao.edu/ sransom/presto/
} 


\section{Dispersion Removal}

As the electromagnetic radiation from a pulsar propagates through the cold and ionized plasma in the Interstellar Medium (ISM), it experiences a frequency-dependent propagation time delay $\Delta t \propto \mathrm{DM} \cdot f^{-2}$, where $\Delta t$ is the time delay, DM or dispersion measure is the integrated free electron column density along the observer's line of sight, and $f$ is the observing frequency. The time delay causes the pulses observed at higher frequencies to arrive earlier than the ones observed at lower frequencies and potentially smears pulsar signals in time.

In order to compensate for this effect, the data were incoherently de-dispersed (i.e. time-shifted and summed) using the PRESTO routine mpiprepsubband. The DM trials ranged from 0 to $350 \mathrm{pc} \mathrm{cm}^{-3}$ for $350 \mathrm{MHz}$ and $820 \mathrm{MHz}$ observations, and from 0 to $1000 \mathrm{pc} \mathrm{cm}^{-3}$ for the $2 \mathrm{GHz}$ observations. This range encompass the predicted DM in the observed directions according to the NE2001 model of the Galactic distribution of free electrons (Cordes \& Lazio 2002). The step sizes of the DM trials were nearoptimally spaced using the routine DDplan.py such that they are small enough to maintain sensitivity to MSP signals at any DM, but large enough to not waste CPU time (see Magro et al. 2011). The de-dispersed time series were Fourier transformed and then searched for periodic signals from pulsars.

\section{Acceleration Searches}

Since most MSPs are in binary systems, neglecting the Doppler effect caused by orbital motion might result in missing MSPs during the search. The effect of binary motion causes a change in the apparent pulse frequency and spreads pulsar signal's power over a number of Fourier bins. As a result, the sensitivity of the search is significantly reduced. The number of Fourier bins which we allow a harmonic to drift 
during the observation, $z$, can be determined as described in Ransom et al. (2001)

$$
z=\frac{A t_{\mathrm{obs}}^{2}}{c P}
$$

where $A$ is the corresponding acceleration caused by the binary orbit, $P$ is the spin period of the pulsar, $c$ is the speed of light, and $t_{\mathrm{obs}}$ is the observing time. We used the routine accelsearch to perform acceleration searches with a maximum $z, z_{\max }$, of 50. accelsearch performed incoherent harmonic summing of the powers of up to 8 harmonics (in powers-of-two) to increase sensitivity to pulsar signals with narrow pulses and also used inter-binning to partially compensate for the scalloped frequency response of FFTs (Ransom et al. 2002).

For highly accelerated pulsars (e.g. pulsars in compact orbits with short orbital periods, such as relativistic binaries and "spider" systems like redbacks and black widows (Roberts 2013)), the drifting in Fourier bins due to acceleration can dramatically smear a pulsar signal over a long observation time. In this case, the pulsar may only be found in searches of short portions of a longer observation, where the Fourier drifting is substantially less (since $z \propto t_{\mathrm{obs}}^{2}$ ). To acquire more sensitivity for these pulsars, we searched all the data in both 5-minute and full-duration searches. All the MSPs in this chapter were found in 5-minute and full-time search or full-time search only, which is not unexpected since all of them are not in tight orbits.

\section{Single-Pulse Searches}

Searching for individual bright pulses provides an approach which is complementary to Fourier methods, since single-pulse searches can identifypulsars which are too faint "on average", but which have a large intensity variations on short (i.e. comparable to the spin period) timescales. Examples of pulsars discovered with single-pulse searches 
are Rotating Radio Transients (RRATs, McLaughlin et al. 2006) and pulsars with socalled giant pulses (Johnston \& Romani 2003).

For all our sources we performed single-pulse searches on the de-dispersed time series using single_pulse_search.py from PRESTO. This routine performs matchedfiltering on the time-series data using boxcars of various widths as templates. All pulse candidates above $(\mathrm{S} / \mathrm{N})_{\min }=5$ threshold were saved for further inspection. The actual flux sensitivity depended on the template width $n_{\text {box }}$ (usually from 1 to 30 samples) and was calculated as follows:

$$
S_{\min }^{\mathrm{sp}}=\frac{(\mathrm{S} / \mathrm{N})_{\min }\left(T_{\mathrm{sys}}+T_{\mathrm{sky}}\right)}{G \sqrt{n_{\mathrm{p}} n_{\mathrm{box}} t_{\mathrm{res}} \Delta f}} .
$$

The resulting pulse sensitivities were $S_{\text {min }}^{\mathrm{sp}} \times \sqrt{n_{\mathrm{box}}}$, or $1.4,0.4$ and $0.2 \mathrm{Jy}$ for 350,820 and $2000 \mathrm{MHz}$ respectively. No single pulses were detected (see Section 2.5.3).

\subsubsection{Pulsar Timing}

The idea of pulsar timing is to create a model of the neutron star rotational behaviour which can precisely predict the arrival times of every pulse from the pulsar. The standard procedure to achieve a pulsar timing model is to iterate and bootstrap a simple initial model based on measured times of arrival (TOAs) using a series of follow-up observations. This iterative process usually results in a "full" timing model (meaning at least an accurate astrometric position, spin frequency, frequency derivative, and Keplerian orbital parameters, if in a binary), within a year.

Following their discovery, each new MSP was part of follow-up timing observations with the GBT using GUPPI in search-mode. The observations were typically at $820 \mathrm{MHz}$, with 2048 channels over $200 \mathrm{MHz}$ of bandwidth and sampled every $61.44 \mu \mathrm{s}$. Occasionally, observations were made at $350 \mathrm{MHz}$ with $100 \mathrm{MHz}$ of bandwidth split 
into 4096 channels and using $81.92 \mu$ s sampling. For each observation, we generated one or more TOAs by cross correlating the pulse profiles, as integrated across observing frequency and time after folding modulo the predicted topocentric pulse period, with a noiseless pulse template based on gaussian fits to the discovery pulse profile, or a subsequently measured one with high $S / N$. For some pulsars, after preliminary phase-connected timing solutions were acquired, we switched to using GUPPI in incoherent fold-mode. Those observations, made at $820 \mathrm{MHz}$, had 2048 frequency channels over $200 \mathrm{MHz}$ of bandwidth, but with $40.96 \mu$ s sampling.

For PSRs J1142+0119 and J1312+0051, we acquired extended timing observations with the Nançay telescope at $1.48 \mathrm{MHz}$ using the NUPPI backend with 1024 channels over $512 \mathrm{MHz}$ bandwidth.

We used the TEMPO ${ }^{2}$ and TEMPO2 ${ }^{3}$ software packages to fit the measured TOAs to timing models which contain astrometric, spin, and binary parameters. For most binary pulsars the DD model (Damour \& Deruelle 1986) is well-suited to describe the orbital parameters. However, for pulsars in highly circular orbits, such that

$$
\frac{a \sin i}{c} e^{2} \ll \frac{T_{\mathrm{res}}}{\sqrt{N_{\mathrm{TOA}}}},
$$

where $a$ is the semi-major axis, $i$ is the inclination angle of the binary, $e$ is the eccentricity, $c$ is the speed of light, $T_{\text {res }}$ is the RMS timing precision, and $N_{\text {TOA }}$ is the number of TOAs, we used the ELL1 timing model (Lange et al. 2001). In that model, the parameters $\epsilon_{1} \equiv e \sin \omega$ and $\epsilon_{2} \equiv e \cos \omega$ are defined, where $\omega$ is the longitude of periastron, and which are much less covariant than $T_{0}$ and $\omega$ in the DD model for circular systems. The orbital phase in the ELL1 model is referenced to the time of

\footnotetext{
${ }^{2}$ http://tempo.sourceforge.net

${ }^{3}$ http://www.sf.net/projects/tempo2
} 
the ascending node, $T_{a s c} \equiv T_{0}-\omega P_{b} /(2 \pi)$, where $T_{0}$ is the epoch of periastron passage and $P_{b}$ is the orbital period.

The observed period derivatives $\left(\dot{P}_{\text {obs }}\right)$ are usually contaminated by an apparent acceleration from a transverse motion of the pulsar (known as the Shklovskii effect (Shklovskii 1970)) and an acceleration because of the Galactic potential towards the Galactic center. These effects cause an underestimation of the intrinsic period derivative $\left(\dot{P}_{\text {int }}\right)$ since

$$
\dot{P}_{\text {int }}=\dot{P}_{\mathrm{obs}}-\dot{P}_{\mathrm{Shk}}-\dot{P}_{\mathrm{Gal}}
$$

and

$$
\dot{P}_{\mathrm{Shk}}=\left(\frac{P}{c}\right) d \mu^{2}
$$

where $\dot{P}_{\text {Shk }}$ is a period derivative term from the Shklovskii effect, $\dot{P}_{\text {Gal }}$ is a period derivative term resulting from the Galactic gravitational potential, $P$ is the pulsar spin period, $c$ is the speed of light, $d$ is the pulsar distance (estimated by the NE2001 Galactic electron density model (Cordes \& Lazio 2002)) and $\mu$ is the proper motion of the pulsar. To calculate $\dot{P}_{\text {Gal }}$, we adopted the Galactic potential model described in Reid et al. (2009). Without these corrections, the underestimated $\dot{P}_{\text {int }}$ leads to the underestimation of physical properties such as spin-down luminosity $\left(\dot{E} \propto \dot{P} / P^{3}\right)$ and surface magnetic field strength $\left(B_{s} \propto \sqrt{P \cdot \dot{P}}\right)$.

Unfortunately, the proper motion is rather difficult to measure from pulsar timing, especially without extended timing baselines. We therefore calculated proper motion upper limits $\left(\mu_{\mathrm{up}}\right)$ for all new MSPs assuming that $\dot{P}_{\text {Shk,max }}=\dot{P}_{\text {obs }}$, where $\dot{P}_{\text {Shk,max }}$ is the maximum $\dot{P}_{\text {Shk }}$. From equation (2.6), the upper limit proper motion, $\mu_{\text {up }}$, is

$$
\mu_{\mathrm{up}}=\sqrt{\frac{\dot{P}_{\mathrm{obs}} c}{P d}}
$$


The values of $\mu_{\mathrm{up}}$, compared to $\mu$ measured by pulsar timing when possible ( $\left.\mu_{\text {timing }}\right)$, are shown on Table 2.2.

\subsubsection{LAT Data Analysis}

This section is contributed by Tyrel Johnson

The LAT is a pair-conversion telescope sensitive to $\gamma$-ray with energies from $20 \mathrm{MeV}$ to $>300 \mathrm{GeV}$ with a 2.4 sr field of view (Atwood et al. 2009). The accuracy with which incoming event directions are reconstructed, or point-spread function $(\mathrm{PSF})$, is dependent on the energy $(E)$, interaction point within the instrument, and angle with respect to the spacecraft $\mathrm{z}$ axis ${ }^{4}(\theta)$. For an event belonging to the SOURCE class converting in the front of the instrument, the $68 \%$ confidencelevel PSF radius, averaged over the acceptance, can be approximated as $\Theta_{68}(E)=$ $\sqrt{\left(0^{\circ} 66(E / 1 \mathrm{GeV})^{-0.76}\right)^{2}+\left(0^{\circ} 08\right)^{2}}$. The total effective area for a near on-axis, $1 \mathrm{GeV}$, SOURCE class $\gamma$-ray is $\sim 7000 \mathrm{~cm}^{2}$. Events triggering the LAT are time stamped using an on-board GPS receiver that is accurate to within $<1 \mu$ s relative to UTC (Abdo et al. 2009c).

For each MSP, we selected LAT P7REP data (Bregeon et al. 2013) corresponding to the SOURCE class recorded between 2008 August 4 and 2013 December 4 within $15^{\circ}$ of the radio position, sufficient to accommodate the tails of the PSF at low energy; energies from 0.1 to $100 \mathrm{GeV}$, the lower limit is that recommended for analysis of P7REP data and the upper limit adequately covers the range of known pulsar cutoff energies; and zenith angles $\leq 100^{\circ}$, to reduce contamination of $\gamma$-ray from the limb of the Earth. Good time intervals were then selected corresponding to when the

\footnotetext{
${ }^{4}$ For more details see http://www.slac.stanford.edu/exp/glast/groups/canda/lat_Performance.htm and Ackermann et al. (2012).
} 
instrument was in nominal science operations mode, the data were flagged as good, and the rocking angle of the spacecraft did not exceed $52^{\circ}$. These good time interval selections allowed us to construct one all-sky exposure cube and binned exposure map for all the MSPs, similar to what was done by (Acero et al. 2015), for example. All LAT analyses were performed using the Fermi Science Tools v9r32p $5^{5}$.

\subsubsection{Joint $\gamma$-ray and Radio Timing}

This section is contributed by Scott Ransom

For each of the MSPs, we used the radio timing ephemerides as well as the fermi plugin for TEMP02 to assign each LAT event to the appropriate rotational phase of the pulsar. This resulted in $\gamma$-ray-pulsation detections for each MSP. For PSRs J0533+6759. J1630+3734, J1858-2216, J2310-0555, and J2042+0246, the $\gamma$-ray pulsations are quite strong, relatively "sharp", and persist for over seven years, significantly longer than our radio timing baselines. For these pulsars, we conducted an MCMC timing analysis using individual gamma-rays to better refine the apparent frequency derivative, and potentially to constrain or measure proper motion.

We applied a likelihood calculation via MCMC for each and every photon over the full Fermi mission and optimized the resulting pulse profile in an iterative manner, similar to that described in Pletsch \& Clark (2015). As in Abdo et al. (2013) we calculate the $\log$ likelihood $\mathcal{L}$ for all $N$ photons (numbered as $j$ and arriving at times $t_{j}$ ), based on an assumed timing model $\mathbf{u}$ comprising many parameters, and an assumed stable gamma-ray pulse profile $F(\phi)$, where $\phi$ is the rotational phase of the pulsar. The area-normalized pulse profile is treated as a probability density function for arriving photons. The known LAT response as functions of energy and position,

\footnotetext{
${ }^{5}$ Available for download at http://fermi.gsfc.nasa.gov/ssc/data/analysis/software/.
} 
in combination with a model gamma-ray sky and model pulsar spectrum, allow us to assign weights $w_{j}$ for each photon, indicating their likelihood of coming from the pulsar in question.

Together, we have

$$
\log \mathcal{L}(\mathbf{u})=\sum_{j=1}^{N} \log \left[w_{j} F\left(\phi_{j}\left(t_{j}, \mathbf{u}\right)\right)+\left(1-w_{j}\right)\right]
$$

which we can then maximize via MCMC techniques while varying the timing parameters in $\mathbf{u}$. Our current implementation is based on emcee (Foreman-Mackey et al. 2013) which uses affine transforms to efficiently explore high-dimensional parameter spaces (like $\mathbf{u}$ ) and map out parameter confidence regions, even when they are highly correlated. The timing model calculations, including relativistic corrections, are performed using the new high-precision timing software PINT ${ }^{6}$, a next-generation high-precision pulsar timing code being developed as independent checks of, and modern improvements upon, the traditional timing packages TEMPO and TEMPO2. The Fermi tools process the photons and transform their time stamps from the location of the spacecraft to the geocenter so that PINT can fit for a variety of astrometric parameters. These single-photon Bayesian MCMC techniques, coupled with PINT, allow us to extract all the useful timing information from each LAT photon.

Our preliminary implementation of this technique, called event_optimize.py, is available as part of PINT. We used it to determine improved spin frequency derivatives (due to the much-longer $\gamma$-ray timing baseline) for the five MSPs J0533+6759, $\mathrm{J} 1630+3734$, J1858-2216, J2042+0246, and J2310-0555, whose frequency derivative from the MCMC analysis deviated by more than $1 \sigma$ from, and was more precise than, the radio timing results.

\footnotetext{
${ }^{6}$ https://github.com/nanograv/PINT
} 


\subsection{Results}

\subsubsection{The New MSPs}

We have phase-connected timing solutions for all twelve MSPs spanning over 7.2 years of observations. One of the MSPs is isolated (J0533+6759), and the remaining MSPs are all "normal" binary MSPs with likely He-WD companions. With our radio timing ephemerides, we folded $\gamma$-ray photons from 7.2 years of Fermi LAT data and found that each of the MSPs exhibits $\gamma$-ray pulsations. We were able to measure proper motions from four MSPs: PSRs J1142+0119, J1312+0051, J1630+3734 and J2042+0246 (see Table. 2.2). The timing residuals for each pulsar are shown in Fig. 2.1 and timing parameters are in Tables 5.1, 5.2, 5.3, 5.4, and 5.5.

The newly discovered pulsars have spin periods in the range of $2.38-5.06 \mathrm{~ms}$ with DMs between 9.3-109.2 pc $\mathrm{cm}^{-3}$. PSRs J2024+0246 and J2310-0555 exhibit strong scintillations at our observing frequency of $820 \mathrm{MHz}$ making them often difficult to detect. All 12 MSPs have rather sharp radio profiles with one to eight components.

\section{PSR J0533+6759}

PSR J0533+6759 is the only isolated MSP in this chapter. It has a spin period of $4.39 \mathrm{~ms}$ and $\mathrm{DM}$ of $57.30 \mathrm{pc} \mathrm{cm}^{-3}$. We determined the $\dot{P}$ of $12.61 \times 10^{-21}$ from the MCMC $\gamma$-ray analysis.

The long integration times used for this survey cause reduced sensitivity to binary pulsars due to their orbital accelerations. While acceleration searches partially mitigate these effects (see section 2.3.2), we are still biased against finding pulsars in compact binaries, and are therefore relatively more sensitive to isolated pulsars. This effect can be seen in the population of MSPs in Globular clusters, which is $\sim 42 \%$ 


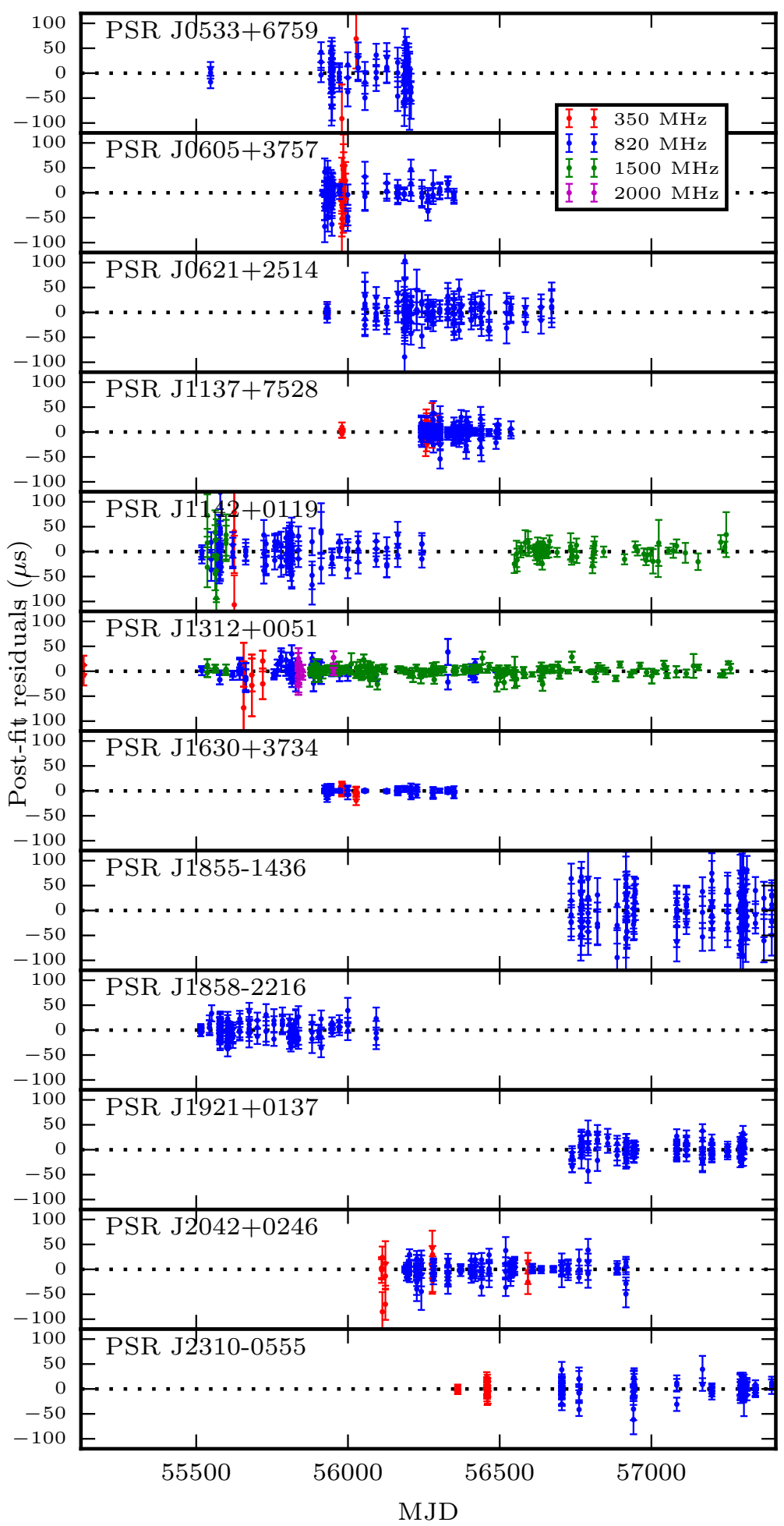

Fig. 2.1.- : Post-fit timing residuals of nine MSPs with timing solutions. The data observed at 350,820 , and $2000 \mathrm{MHz}$ were taken at the GBT, while the data observed at $1500 \mathrm{MHz}$ were taken at the Nançay telescope. 
isolated $^{7}$, compared to $\sim 25 \%$ in the field ${ }^{8}$, due to hours-long integration times. Our searches are 45-50 minutes for each source, much longer than the few-minute integrations of all-sky surveys. Despite this bias in favor of finding isolated MSPs, there have been only seven isolated MSPs found in searches of Fermi unassociated sources $(\sim 10 \%)$. The lack of isolated MSPs may be related to the large numbers of energetic "spider" pulsars (i.e. black widow or redback systems (Roberts 2013)) uncovered in these searches. Those systems, likely ablating away their companions, may be the evolutionary predecessors for isolated MSPs. This implies that Fermi LAT MSPs are, on average, younger than the typical MSP.

\section{PSR J0621+2514}

PSR J0621+2514 has a spin period of $2.72 \mathrm{~ms}$, a DM of $83.64 \mathrm{pccm}^{-3}$, a minimum He-WD companion mass of $0.15 M_{\odot}$ and an orbital period of 1.26 days, the shortest orbital period among the MSPs in this chapter. In addition, this pulsar has a relatively high spin-down luminosity $\left(4.7 \times 10^{34} \mathrm{ergs} \mathrm{s}^{-1}\right)$.

\section{PSR J1142+0119}

PSR J1142+0119 has an extremely small eccentricity that cannot yet be measured from pulsar timing. It has a spin period of $5.1 \mathrm{~ms}$, a DM of $19.2 \mathrm{pccm}^{-3}$, a HeWD companion minimum mass minimum limit of $0.15 M_{\odot}$ and an orbital period of 1.58 days. Despite a timing baseline of 4.7 years, we have only a weak measurement of proper motion of $105 \pm 62$ mas yr$^{-1}$. Given a distance of $0.9 \mathrm{kpc}$, and Equation

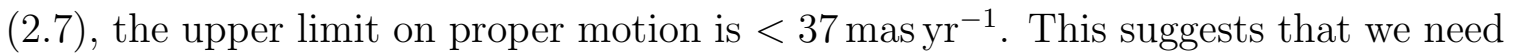
a longer pulsar timing baseline in order to measure a significant proper motion. It

\footnotetext{
${ }^{7}$ From http://www.naic.edu/ pfreire/GCpsr.html

${ }^{8}$ From http://astro.phys.wvu.edu/GalacticMSPs/GalacticMSPs.txt
} 
also suggests that the DM-distance may be underestimated.

\section{PSR J1312+0051}

PSR J1312+0051 has a spin period of $4.23 \mathrm{~ms}$, a DM of $15.35 \mathrm{pc} \mathrm{cm}^{-3}$, a minimum He-WD companion mass of $0.18 M_{\odot}$, and an orbital period of 38.5 days. With the long radio timing span of over five years, we were able to measure significant proper

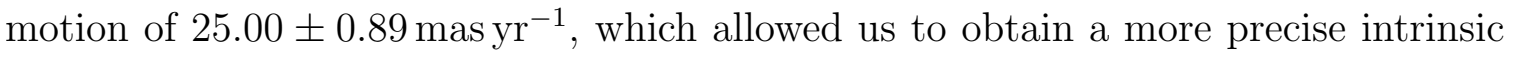
period derivative of $12.0 \times 10^{-21}$ by correcting for Shklovskii effect at a distance of $0.8 \mathrm{kpc}$.

PSR J1312+0051 is bright and well-timed, with a relatively low timing RMS of $4.8 \mu \mathrm{s}$ (low given that most of the timing data were taken in search-mode at the GBT). It is in the Arecibo declination range and has been included in the NANOGrav pulsar timing array (e.g. The NANOGrav Collaboration et al. (2015)).

\section{PSR J1630+3734}

PSR J1630+3734 is also a MSP in a binary system with a He-WD companion. It has a spin period of $3.32 \mathrm{~ms}$, a DM of $14.13 \mathrm{pc} \mathrm{cm}^{-3}$, a minimum companion mass limit of $0.24 M_{\odot}$ and an orbital period of 12.53 days. Using a more accurate $\dot{P}$ of $10.77 \times 10^{-21}$ from the longer-term MCMC $\gamma$-ray timing analysis enabled a radio

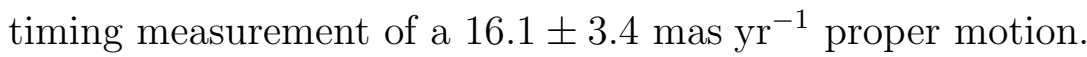

The relatively low timing RMS of $3.6 \mu \mathrm{s}$, might make this pulsar a candidate for NANOGrav. However, the declination of $37^{\circ}$ is near the limit of the declination range for the Arecibo telescope. J1630+3734 should be a great candidate for a future high sensitivity pulsar timing array using the Five-hundred-meter Aperture Spherical radio Telescope (FAST) or the Square Kilometer Array (SKA). 
PSR J1921+0137

PSR J1921+0137 is the latest discovery in this chapter (2014 March) with a spin period of $2.49 \mathrm{~ms}$ and DM of $104.9 \mathrm{pc} \mathrm{cm}^{-3}$, which is the highest among the MSPs in the chapter, a minimum He-WD companion mass of $0.24 M_{\odot}$ and an orbital period of 9.9 days. There is no proper motion measurement from the MCMC analysis or pulsar timing for J1921+0137 yet.

\section{PSR J2042+0246}

PSR J2042+0246 has the longest orbital period (77.2 days) in this chapter. It has a spin period of $4.53 \mathrm{~ms}$, a DM of $9.27 \mathrm{pc} \mathrm{cm}^{-3}$, and a minimum He-WD companion mass limit of $0.19 M_{\odot}$. We measured a significant proper motion of $20.62 \pm 1.90{\text { mas } \mathrm{yr}^{-1}}^{-1}$ from pulsar timing, which led to a more precise measurement of the intrinsic period derivative, after correcting for the Shklovskii effect, of $11.7 \times 10^{-21}$. This pulsar scintillates strongly during observations at $820 \mathrm{MHz}$ due to its low DM, making it often quite difficult to detect.

\section{PSR J2310-0555}

PSR J2310-0555 has a spin period of $2.61 \mathrm{~ms}$, a DM of $15.5 \mathrm{pc} \mathrm{cm}^{-3}$, and an orbital period of 1.4 days. This pulsar was fairly challenging to time due to strong scintillation and a huge difference between an original LAT position and true timing position. The pulsar was found with the GBT at $350 \mathrm{MHz}$ and follow-up timing observations were conducted at $820 \mathrm{MHz}$ (the $820 \mathrm{MHz}$ beam FWHM is $16^{\prime}$ and the $350 \mathrm{MHz}$ beam FWHM is $\left.36^{\prime}\right)$. However, the follow-up timing observations could not detect the pulsar, despite multiple sets of "gridding" observations designed to determine the pulsar's position more precisely. By using an improved source position from the 
3FGL catalog, new $820 \mathrm{MHz}$ observations detected J2310-0555 10.8' away from the original LAT $\gamma$-ray position. In addition, the original position is $9.7^{\prime}$ away from the timing position.

PSRs J0605+3757, J1137+7528, J1855-1436 and J1858-2216

PSRs J0605+3757, J1137+7528, J1855-1436, and J1858-2216 are MSPs with typical He-WD companions with spin period ranges from 2.4 to $3.6 \mathrm{~ms}$ and DM ranges from

20.9 to $109.2 \mathrm{pc} \mathrm{cm}^{-3}$. They have a relatively long orbital periods, ranging from 29.2 to 61.2 days. We were unable to measure either $\dot{P}$ from the MCMC analysis nor proper motion from radio timing for these pulsars.

\subsubsection{Radio and Gamma-ray Light Curves}

This section is contributed by Tyrel Johnson

The radio and the $\gamma$-ray components of pulsar emission are believed to originate mostly in different locations in the magnetosphere (Venter et al. 2009). The $\gamma$-ray emission mechanism is typically thought to be curvature radiation from particles accelerated along the magnetic field lines in the outer magnetosphere (e.g. Muslimov \& Harding 2004; Cheng et al. 1986); however, other models predict an additional synchrotron component (Viganò et al. 2015), that the emission is all synchrotron from the striped pulsar wind (Pétri 2012), or that the emission arises from inverse Compton scattering (Lyutikov et al. 2012). While much is known about pulsar radio emission, the exact mechanism is still being debated. Although it is likely that for MSPs, the radio emission is generated much farther out in the magnetosphere than is thought to be the case for slow pulsars.

By analysing phase differences between radio and $\gamma$-ray light curves (LCs), Venter 
Table 2.2. The Upper Limit and Measured Proper Motions

\begin{tabular}{cccc}
\hline \hline PSR & $\begin{array}{c}d \\
(\mathrm{kpc})\end{array}$ & $\begin{array}{c}\mu_{\mathrm{up}} \\
\left(\mathrm{mas} \mathrm{yr}^{-1}\right)\end{array}$ & $\begin{array}{c}\mu_{\text {timing }} \\
\left(\text { mas yr }^{-1}\right)\end{array}$ \\
\hline $\mathrm{J} 0533+6759$ & 2.4 & 22 & unknown \\
$\mathrm{J} 0605+3757$ & 0.7 & 32 & unknown \\
$\mathrm{J} 0621+2514$ & 2.3 & 40 & unknown \\
$\mathrm{J} 1137+7528$ & 1.5 & 19 & unknown \\
$\mathrm{J} 1142+0119$ & 0.9 & 37 & $105 \pm 62$ \\
$\mathrm{~J} 1312+0051$ & 0.8 & 46 & $25.0 \pm 1$ \\
$\mathrm{~J} 1630+3734$ & 0.9 & 39 & $16.1 \pm 3$ \\
$\mathrm{~J} 1855-1436$ & 3.1 & 20 & unknown \\
$\mathrm{J} 1858-2216$ & 0.9 & 27 & unknown \\
$\mathrm{J} 1921+0137$ & 3.5 & 30 & unknown \\
$\mathrm{J} 2042+0246$ & 0.8 & 40 & $20.6 \pm 2$ \\
$\mathrm{~J} 2310-0555$ & 0.7 & 33 & unknown \\
\hline
\end{tabular}
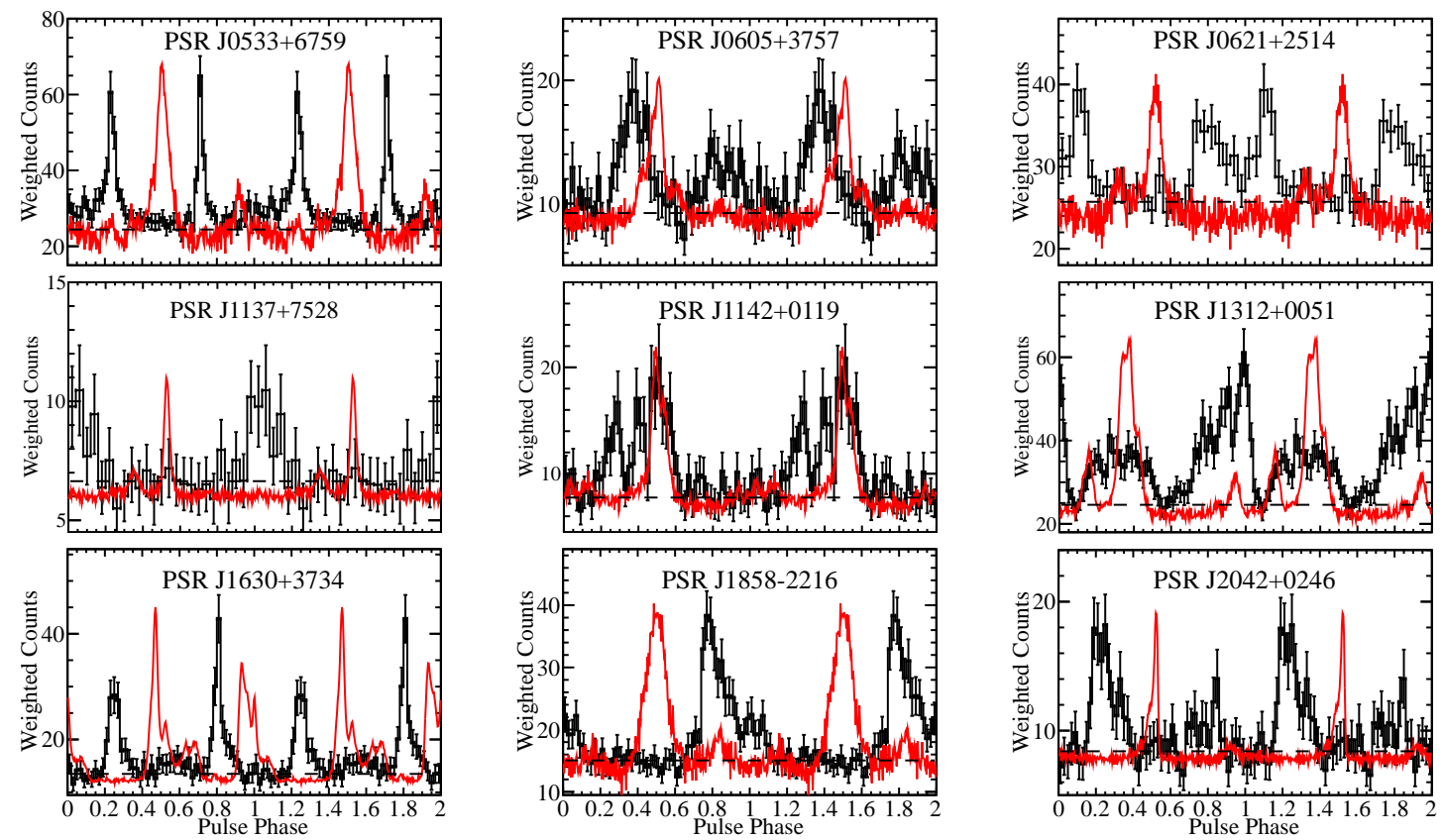

Fig. 2.2.-: The $\gamma$-ray and radio light curves of nine MSPs. The radio profiles are summed over detections at $820 \mathrm{MHz}$ while $\gamma$-ray light curves correspond to the integrated Fermi LAT photons found within $5^{\circ}$ of each MSP, and with spectrally and positionally determined photon weights. The horizontal dashed lines are an estimation of the background level. 
et al. (2012) and Johnson et al. (2014) divided MSPs into three broad classes. For Class I the $\gamma$-ray peak trails the radio peak by $<0.5$ in phase, for Class II the radio and $\gamma$-ray peaks are aligned (within 0.1 in phase), and for Class III, the $\gamma$-ray peak leads (between 0.3 and 0.1 in phase) the radio peak. After fitting various pulsar emission models to the radio and $\gamma$-ray MSP LCs, they found that the radio emission of Class I and III MSP most probably originates in the open field region above the polar cap near the stellar surface. The $\gamma$-ray emission of Class I MSP comes from narrow vacuum gap near the surface of the last closed field lines. Class III high energy emission is thought to come from the high altitude region above the polar cap. For Class II MSPs, the radio and $\gamma$-ray emission are both assumed to originate in the extended altitude region close to the last open magnetic field line.

In our work the $\gamma$-ray profiles were folded with the radio ephemerides and the radio delay due to propagation in the ISM was removed. Therefore, the lags between radio and $\gamma$-ray profiles are physical. The radio and $\gamma$-ray LCs are shown in Fig. 2.2. Note that we only have preliminary $\gamma$-ray results for PSRs J1855-1436, J1921+0137 and J2310-0555 so we only present nine MSPs here. The analysis will be completed within the next few months after this thesis. For nine MSPs we performed the preliminary classification by visual examination of the lags between radio and $\gamma$-ray LCs. The results are the following:

Class I: PSRs J0533+6759，J0621+2514，J1137+7528，J1312+0051，J1630+3734 and J1858-2216.

Class II: None.

Class III: PSRs J0605+3757, J1142+0119 and J2042+0246

However, a fitting analysis as in Johnson et al. (2014) should be applied to deter- 
mine the lags between $\gamma$-ray and radio LCs more precisely.

\subsubsection{Gamma-ray Pulsations}

This section is contributed by Tyrel Johnson

For the twelve MSPs with phase-connected timing solutions, a binned maximum likelihood analysis was performed on a $21.2 \times 21^{\circ} 2$ (the largest square that will fit entirely inside our $15^{\circ}$ radius selection) region of interest (ROI), centered on the corresponding radio position, using the P7REP_SOURCE_V15 instrument response functions (IRFs). To model the ROI for each MSP, we included sources from the LAT 4-year source catalog, 3FGL (Acero et al. 2015), within a $25^{\circ}$ radius of the ROI center. All known, extended sources in 3FGL were modeled as such using the same spatial templates. The Galactic diffuse emission was modeled using the gll_iem_v05r1.fits spatial template and the isotropic diffuse (including the extra-Galactic $\gamma$-ray and residual instrument backgrounds) using iso_source_v05.txt ${ }^{9}$

Because of our choice of good time intervals, it was necessary to model the Earth limb emission using the same spatial template and spectral shape as 3FGL. PSRs J0533+6759, J0604+3757, J1142+0119, J1312+0051, J1630+3734, and J1858-2216 were included in the 3FGL catalog as known $\gamma$-ray pulsars, but we moved each to their respective radio timing positions in our model, requiring changing the positions by $\leq 0$ 0.1. PSRs J0621+2514, J1137+7528, and J2042+0246 were not known $\gamma$-ray pulsars in 3FGL and we thus found the closest sources, moved those to the radio timing positions, and identified them in our model, requiring changing the positions by $\leq 0$.04. In each model, the spectral parameters of point and extended sources within $6^{\circ}$ of the ROI center and with test statistic (TS, Acero et al. 2015) values $\geq 50$ were

\footnotetext{
${ }^{9}$ These diffuse models are available for download at http://fermi.gsfc.nasa.gov/ssc/data/access/lat/BackgroundModels.html.
} 
left free, as were the normalizations of the Galactic and isotropic diffuse components. All other source parameters were kept fixed throughout the analysis.

We modeled the spectrum of each MSP as a simple power law (Eq. 2.8) and an exponentially-cutoff power law (Eq. 2.9) with the $b$ parameter fixed to one (a simple cutoff) and free to vary.

$$
\begin{gathered}
\frac{d N}{d E}=N_{0}\left(\frac{E}{E_{0}}\right)^{-\Gamma} \\
\frac{d N}{d E}=N_{0}\left(\frac{E}{E_{0}}\right)^{-\Gamma} \exp \left\{-\frac{E^{b}}{E_{\mathrm{C}}}\right\} .
\end{gathered}
$$

In Eqs. 2.8 and 2.9, $N_{0}$ is a normalization factor with units $\mathrm{cm}^{-2} \mathrm{~s}^{-1} \mathrm{MeV}^{-1}, \Gamma$ is the photon index, and $E_{0}$ is a scale parameter in units of $\mathrm{MeV}$, taken as the pivot energy from 3FGL. For PSRs J0621+2514, J1137+7528, and J2042+0246 we used the pivot energy from the closest 3FGL sources that we moved to the radio positions. In Eqn. $2.9, E_{\mathrm{C}}$ is the cutoff energy in units of $\mathrm{GeV}$ and $b$ is an exponential index controlling the sharpness of the cutoff.

We then calculated $\mathrm{TS}_{\text {cut }}=-2\left(\ln \left(\mathcal{L}_{\text {cut }}\right)-\ln \left(\mathcal{L}_{\mathrm{pl}}\right)\right)$ and $\mathrm{TS}_{\mathrm{b}}$ free $=-2\left(\ln \left(\mathcal{L}_{\mathrm{b} \text { free }}\right)-\right.$ $\ln \left(\mathcal{L}_{\text {cut }}\right)$ ), where $\mathcal{L}_{\text {pl }}$ is the maximum likelihood of the power-law fit, $\mathcal{L}_{\text {cut }}$ is the maximum likelihood of the cutoff fit with $b=1$, and $\mathcal{L}_{\mathrm{b}}$ free is the maximum likelihood of the cutoff fit with the $b$ parameter free to vary. In each case, there is one additional degree of freedom, allowing us to use the likelihood ratio test to approximate the rejection of the power law in favor of the simple exponential cutoff as $\sim \sqrt{\mathrm{TS}_{\mathrm{cut}}}$ and the rejection of the simple cutoff in favor of the $b$-free fit as $\sim \sqrt{\mathrm{TS}_{\mathrm{b}} \text { free }}$.

The best-fit results for each MSP are given in Tables 5.1, 5.2, 5.3, 5.4, and 5.5. For all but PSR J1137+7528, the power-law spectrum was rejected in favor of the simple cutoff at $\gtrsim 4 \sigma$. The $b$-free fit was never significantly preferred over the simple exponential cutoff (always $\lesssim 2 \sigma$ ). For PSR J1137+7528, the power-law shape can 
not be significantly rejected in favor of a simple exponential cutoff and thus we only report values for and derived from the power-law fit. For each MSP we provide the spectral parameters and point-source TS from the best fit, the values of $\mathrm{TS}_{\text {cut }}$ and $\mathrm{TS}_{\mathrm{b} \text { free, }}$ and the integrated photon $(F)$ and energy fluxes $(G)$ from 0.1 to $100 \mathrm{GeV}$ are derived from the best fit.

As estimated by (Ackermann et al. 2012), the systematic uncertainties on the LAT effective area $\left(A_{\text {eff }}\right)$ are $10 \%$ for $\log _{10}(E / 1 \mathrm{MeV}) \leq 2,5 \%$ for $\log _{10}(E / 1 \mathrm{MeV})=$ 2.75 , and $10 \%$ for $\log _{10}(E / 1 \mathrm{MeV}) \geq 4$ with linear extrapolation, in log space, in between. Following Abdo et al. (2013), we estimate the effects of these uncertainties on our derived spectral parameters by generating bracketing IRFs using a modified

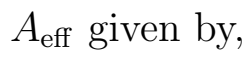

$$
A_{\text {brack }}(E, \theta)=A_{\text {eff }}(E, \theta)(1+\operatorname{err}(E) B(E))
$$

where $\operatorname{err}(E)$ represented the systematic uncertainties noted previously and the bracketing functions we used were $B(E)= \pm 1$ to estimate systematic uncertainties on $N_{0}$ and $B(E)= \pm \tanh \left(\log _{10}\left(E / E_{0}\right) / 0.13\right)$ to estimate systematic uncertainties on both $\Gamma$ and $E_{\mathrm{C}}$. For the latter bracketing functions, we generated bracketing IRFs specifically for each MSP using $E_{0}$ from the four-year source list. In order to estimate the systematic uncertainties on the photon and energy fluxes, we recalculated these values from each bracketing IRFs fit and took the maximum excursions from the nominal values.

When fitting with the bracketing IRFs, we generated new source maps files ${ }^{10}$ for the free point sources in each region of interest with the bracketing IRFs and used the source maps for the fixed sources and the diffuse backgrounds calculated with the P7REP_SOURCE_V15 IRFs. This was done as the diffuse backgrounds are

\footnotetext{
${ }^{10}$ For more information on source maps files for use with LAT binned likelihood analysis see http: //fermi.gsfc.nasa.gov/ssc/data/analysis/scitools/binned_likelihood_tutorial.html.
} 
specific to the LAT data and nominal IRFs and the spectral values of the fixed sources correspond to the same IRFs.

In order to get the best $\gamma$-ray pulse profiles possible, we extended the data set to 2015 June 24, the end of the P7REP data, for the timing analysis. We selected events in this time range with reconstructed directions within $5^{\circ}$ of each MSP and used the best-fit spectral model to calculate the probability for each event to be associated with that particular MSP. We then folded the events at the radio period using the fermi plugin (Ray et al. 2011) for the TEMPO2 timing software (Hobbs et al. 2006) and calculated the spectrally-weighted H-test significance (Kerr 2011), resulting in a $>5 \sigma$ pulsed detection for each MSP. The uncertainties for each bin of the $\gamma$-ray light curve and the background level are calculated as described in Guillemot et al. (2012) and Abdo et al. (2013). Pulsed statistics for each MSP are given in Tables 5.1, 5.2, $5.3,5.4$, and 5.5 .

\subsection{Discussion}

\subsubsection{Radio and $\gamma$-ray Flux Densities}

In the early searches for pulsars in Fermi unassociated sources, the bright sources with pulsar-like spectra were intensively searched with various radio telescopes and yielded many discoveries of MSPs (e.g. Ransom et al. 2011, Kerr 2011). However, from the more recent searches, some bright radio MSPs were found in fainter Fermi unassociated sources (e.g. Bhattacharyya et al. 2013). These detections suggest that there may be no, or only weak correlations between the flux of the pulsars and the $\gamma$-ray sources. With additional $12 \gamma$-ray MSPs in this chapter, we have more samples to investigate the correlation between radio flux densities and energy fluxes of $\gamma$-ray 
MSPs.

Figure 2.3 is a plot of Fermi $\gamma$-ray MSPs, showing radio flux densities at $1400 \mathrm{MHz}$ against $\gamma$-ray energy fluxes from photons with energies above $100 \mathrm{Mev}$. The pulsars on the plot are from the second $\gamma$-ray pulsar catalog (2PC) (Abdo et al. 2013), another Fermiunidentified source analysis (Camilo et al. 2015) and the new GBT Fermi MSPs in this chapter. For MSPs with flux-calibrated data, we used those values as starting points. If they had no flux calibration measurements, we used the radiometer equation to calculate flux densities at $820 \mathrm{MHz}$. Then, we extrapolated the $1400 \mathrm{MHz}$ flux densities from the flux density estimates at $820 \mathrm{MHz}$ using the relation $S_{f} \propto f^{\alpha}$, where $\alpha$ is the spectral index. We adopted a spectral index of -1.41 from the latest study of the distribution of pulsar spectral indices (Bates et al. 2013). Furthermore, we adopted $\gamma$-ray energy fluxes from the third Fermi LAT source catalog (3FGL) (Acero et al. 2015). Figure 2.3, shows no correlation between the radio and $\gamma$-ray fluxes and suggests that faint LAT unassociated sources are as reasonable MSP candidates as the bright ones. Additionally, it shows that the new GBT Fermi MSPs are among the faintest $\gamma$-ray Fermi MSPs detected in the radio. This emphasizes the high sensitivity of the GBT in discovering faint MSPs.

\subsubsection{Galactic Plane Searches with S-band}

Young and energetic pulsars are believed to be the primary $\gamma$-ray emitters near the Galactic plane. We have observed 22 pulsar-like Galactic plane sources and found no new pulsars. The unsuccessful searches in the plane are not completely unexpected. As discussed in Abdo et al. (2013), the poor success rate of radio searches near the Galactic plane is probably due to the combination of smaller radio beam sizes in young pulsars compared to MSPs (and to $\gamma$-ray beams) and the intensive radio 


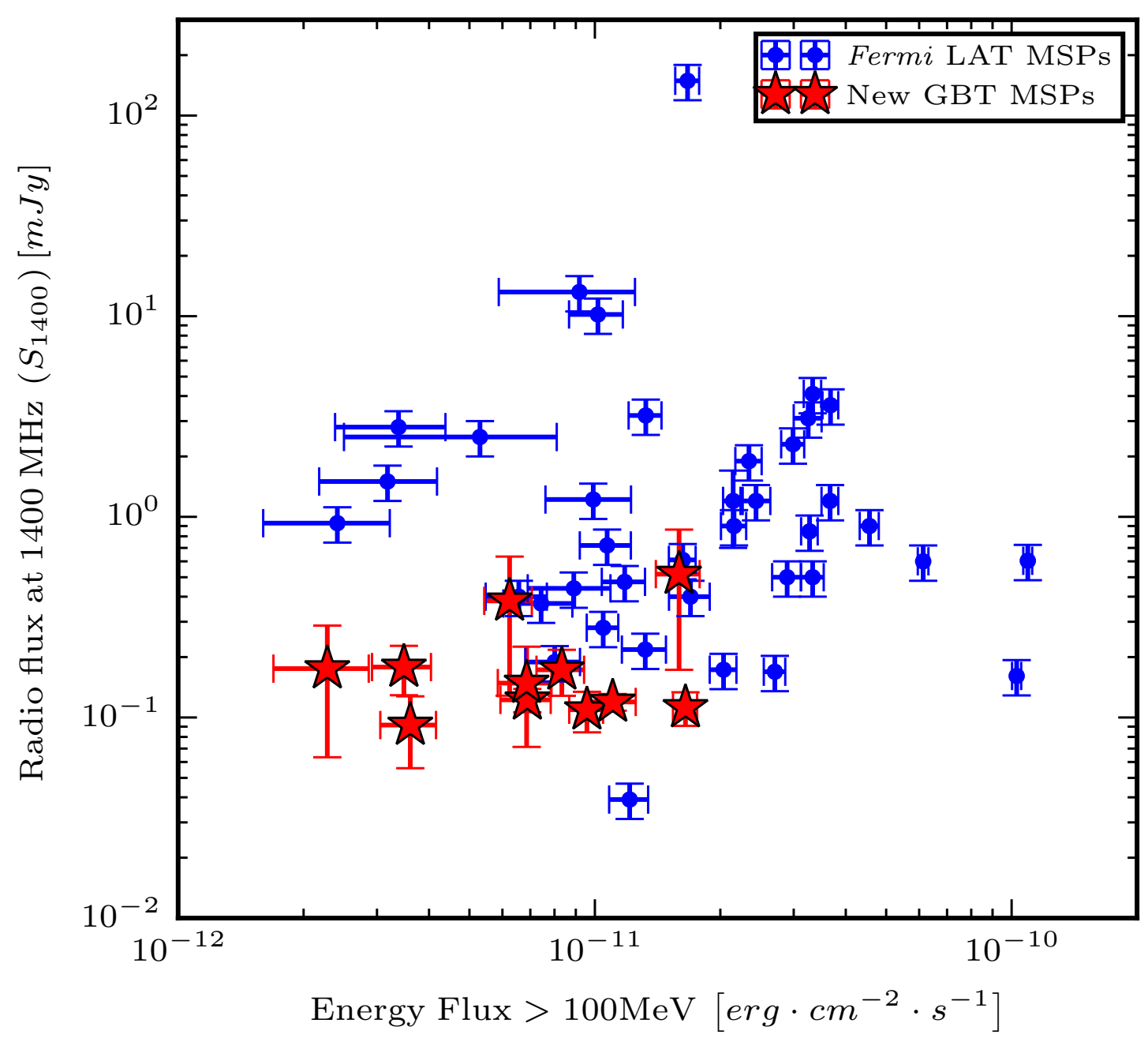

Fig. 2.3.- : A plot of radio flux density vs $\gamma$-ray flux for the MSPs from the Second Pulsar Catalog (2PC) (Abdo et al. 2013) and the new GBT MSPs. The lack of any correlation between the radio and gamma-ray fluxes likely explains much of the continuing success of the radio MSP searches of Fermi unassociated sources, and suggests that many more MSPs are waiting to be found based on even a very weak $\gamma$-ray signature. 
searches of previous pulsar surveys. In addition, the large $95 \%$ error regions of most Fermi unassociated sources are more confused in the crowded regions of the Galactic plane, and are significantly larger than the GBT's S-band beam width of 6-arcmin. To cover an entire $\gamma$-ray error region, we have to perform multiple short-time gridded observations on most sources which reduces the search sensitivity. It is also possible that pulsars in the field may have steep spectral indices.

\subsubsection{Single-Pulse Searches}

We found no individual pulses from any of the 198 sources. As discussed in Deneva et al. (2009), the intermittency ratio, the measurement of the efficiency to detect objects by single-pulse search over periodicity search, for MSPs is small. Additionally, all the MSPs on this chapter are not close to the known giant pulse (GP, an occasional intense short-duration burst) emitters as shown on Figure 2.4, the $B_{L C}-\dot{E}$ correlation (Knight 2006). This and the fact that GP emission is likely rare among the MSP population, largely explains why single-pulse searches are not successful towards LAT unassociated sources.

\subsubsection{LAT $\gamma$-ray Detection Threshold}

Abdo et al. (2013) discuss the prediction that a metric for $\gamma$-ray luminosity is related to spin-down luminosity and distance by $\propto \sqrt{\dot{E}} / D^{2}$. Despite the unreliable distances from the NE2001 model and/or Galactic background confusion from some pulsars, the detectability metric threshold of approximately $10^{16}\left(\mathrm{erg} \cdot \mathrm{s}^{-1}\right)^{\frac{1}{2}} \cdot \mathrm{kpc}^{-2}$ appears to be a good prediction for LAT pulsation detection. Figure 2.5) shows that PSR J2310-0555 falls slightly below the threshold but the rest of the MSPs are above the detectability threshold. 


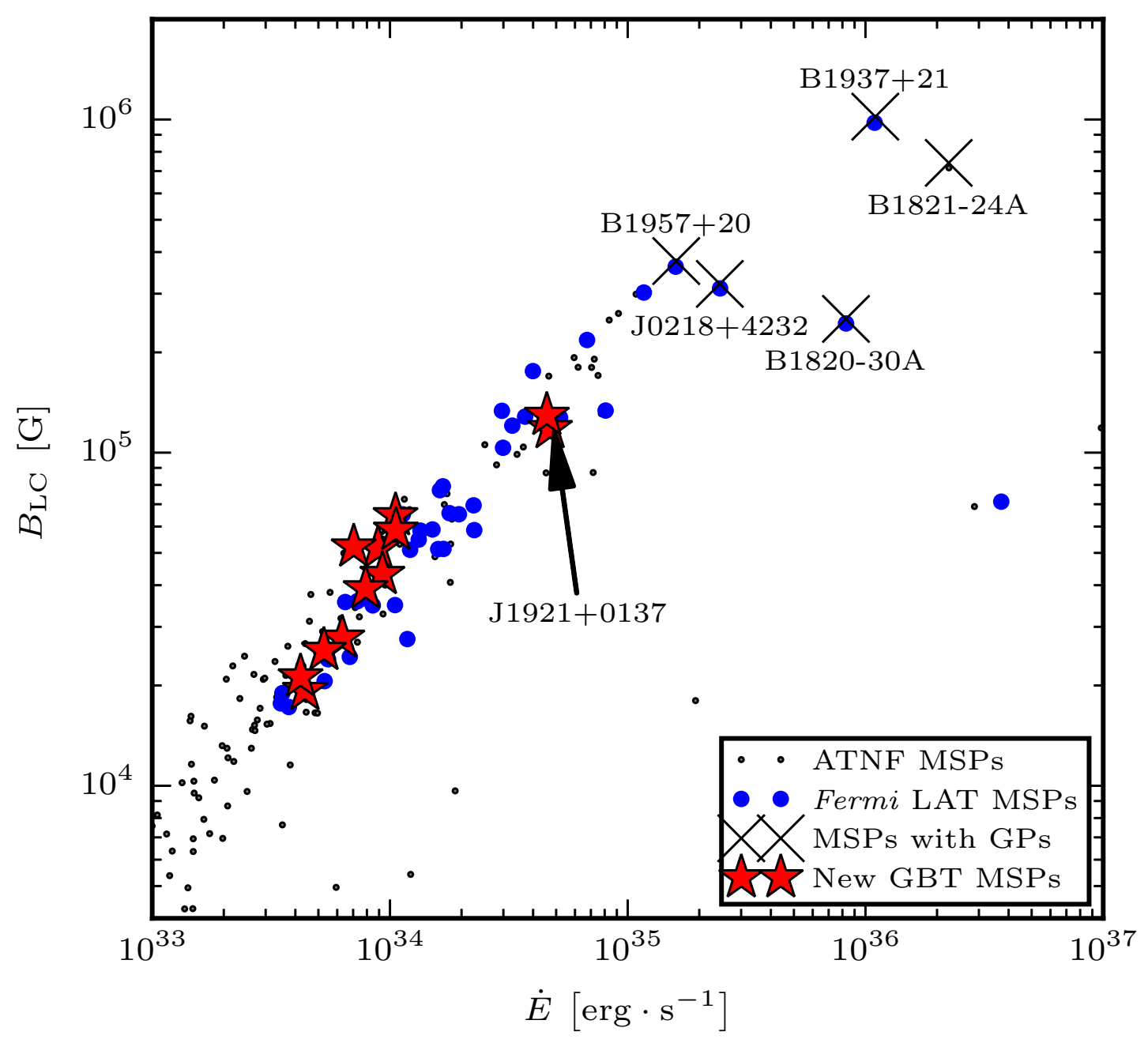

Fig. 2.4.- : The correlation between magnetic field strength at the light cylinder $\left(B_{L C}\right)$ and spin down luminosity $(\dot{E})$ of MSPs, with giant-pulse emitters shown as crosses. 


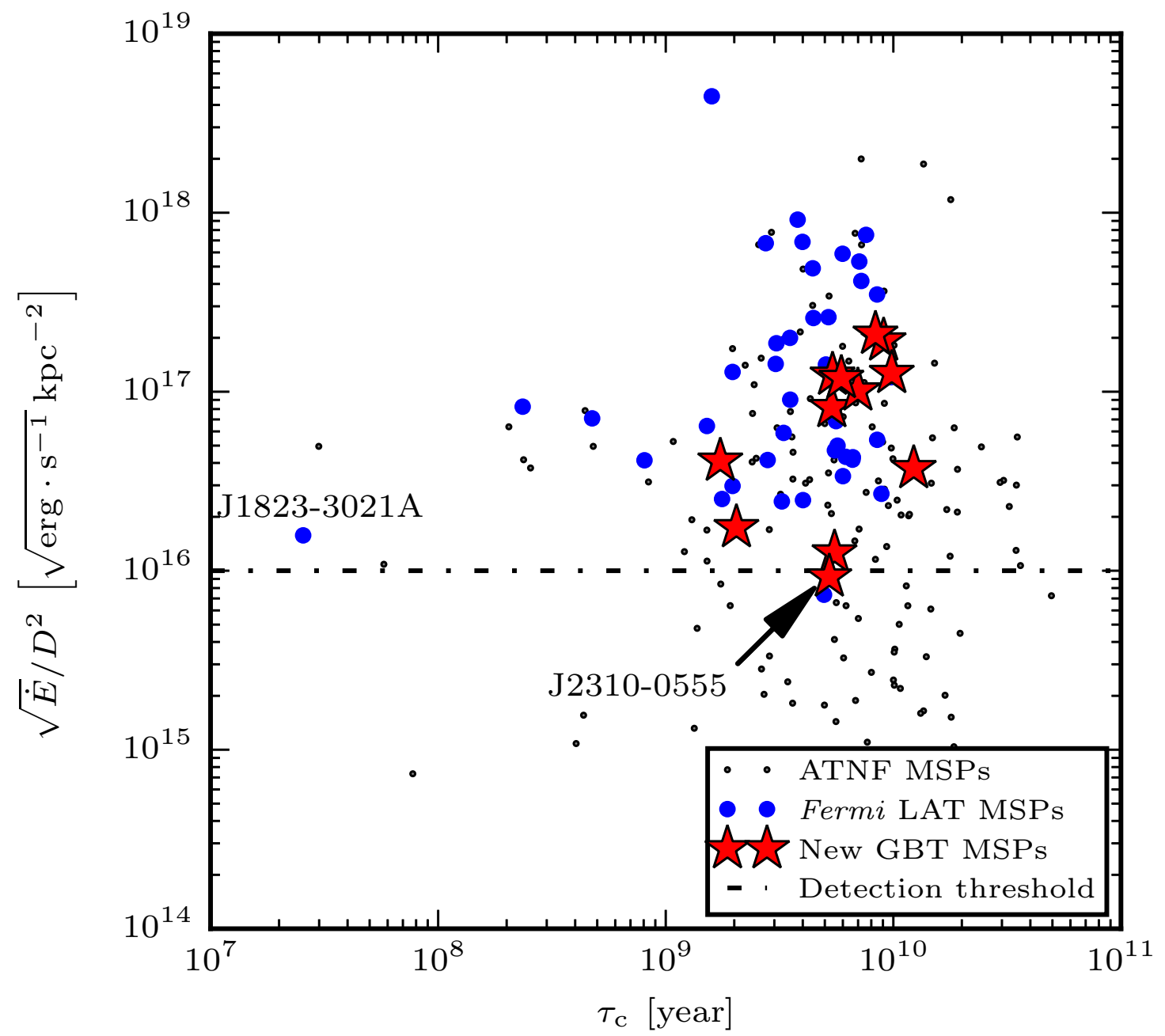

Fig. 2.5.-: $\sqrt{\dot{E}} / D^{2}$ versus characteristic age of ATNF MSPs, LAT MSPs, and the twelve new MSPs described here. The dashed line is a $\gamma$-ray detection threshold at $10^{-16}\left(\mathrm{erg} \cdot \mathrm{s}^{-1}\right)^{\frac{1}{2}} \cdot \mathrm{kpc}^{-2}$. The plot shows PSR J1823-3021A, the most $\gamma$-ray luminous and youngest MSP to date (Freire et al. 2011). 


\subsubsection{Orbital Period and Companion Mass Relation}

This section is contributed by Scott Ransom

MSPs in binary systems are thought to evolve from low-mass X-ray binaries (LMXBs), where the neutron star accretes mass and angular momentum from the companion star (Alpar et al. 1982). The mass transfer process in the system initiates when the donor star evolves up to the red giant branch (RGB), expands to fill its Roche lobe, and stellar materials overflow onto the neutron star through an accretion disk. Based on previous studies of LMXBs with low-mass $\left(<2 M_{\odot}\right)$ main-sequence donors (e.g. Webbink et al. (1983); Joss et al. (1987); Rappaport et al. (1995)), Tauris \& Savonije (1999) derived a relationship, hereafter called TS99, which predicts degenerate He-core white dwarf (He-WD) companion masses from the final orbital period of "normal" (final orbital period $\gtrsim 1-2$ days) binary MSPs.

Accurately measuring companion masses is difficult. Since TS99 was introduced, some fairly precise measurements of He-WD companion masses from either Shapiro delay (via pulsar timing, e.g. PSR J1909-3744, Jacoby et al. (2005); PSR J0437-4751, Verbiest et al. (2008)) or modeling of optical properties of the companion stars (e.g. van Kerkwijk et al. (2005); Kaplan et al. (2014)) have supported the TS99 correlation (e.g. Tauris \& van den Heuvel (2014)). Recently, Fermi and other pulsar surveys have significantly increased the number of known Galactic MSP binaries. By statistically analyzing the binary MSP orbital parameters, we can constrain the He-WD companion masses and therefore test TS99 and/or assumptions about the orbital inclination angle distribution.

For the analysis, we have primarily gathered Galactic MSPs with He-WD companions from the Australian Telescope National Facility (ATNF) pulsar catalog version 
$1.52^{11}$ (Manchester et al. 2005), as well as Fermi MSPs from this and two other recent papers. From the ATNF catalog, we selected only those pulsars unassociated with globular clusters (parameter assoc $\neq$ "GC"), have a short spin period (parameter $P_{0}<30 \mathrm{~ms}$ ), and that have a He-WD companion (parameter BinComp = "He"). We also included pulsars where MinComp = "unknown" with minimum companion mass $<0.4 M_{\odot}$. These pulsars are likely to have a He-WD companion rather than a heavy CO-WD companion. We explicitly excluded known "redback" pulsars, whose companions are non-degenerate and have not yet evolved into He-WDs. These pulsars are listed in Ray et al. (2012) and Roberts et al. (2015). We also ignored J1933-6211, which requires a pulsar mass $<1 M_{\odot}$ to be consistent with TS99. In addition to the ATNF pulsars and the 12 binary MSPs in this chapter, we have included 7 new Fermi MSPs from the PSC: 4 from Parkes (Camilo et al. 2015) and 3 from the GBT observations at $350 \mathrm{MHz}$ (Bangale et al. 2015, in prep). This brings the total number of pulsars in the following analysis to 81.

Figure 2.6 shows the TS99 relation for the 81 Galactic MSPs described above, plus an additional five which have orbital periods shorter than one day For this plot we have assumed a pulsar mass $\left(M_{p}\right)$ of $1.48 M_{\odot}$ as suggested by Özel et al. (2012), for the mean of the recycled neutron star mass distribution. With this assumption, 76 of the 81 MSPs have companion mass ranges consistent with the TS99 relation. There are several possible explanations for the five MSPs which are inconsistent with the TS99 relation, as discussed in Guillemot \& Tauris (2014). For example, they may have evolved from binary systems other than LMXBs (i.e. intermediate mass X-ray binaries (IMXBs)), or they may simply have pulsar masses that differ significantly from $1.48 M_{\odot}$.

A common assumption when investigating binary pulsars and their correspondence

${ }^{11}$ http://www . atnf .csiro.au/people/pulsar/psrcat/. 


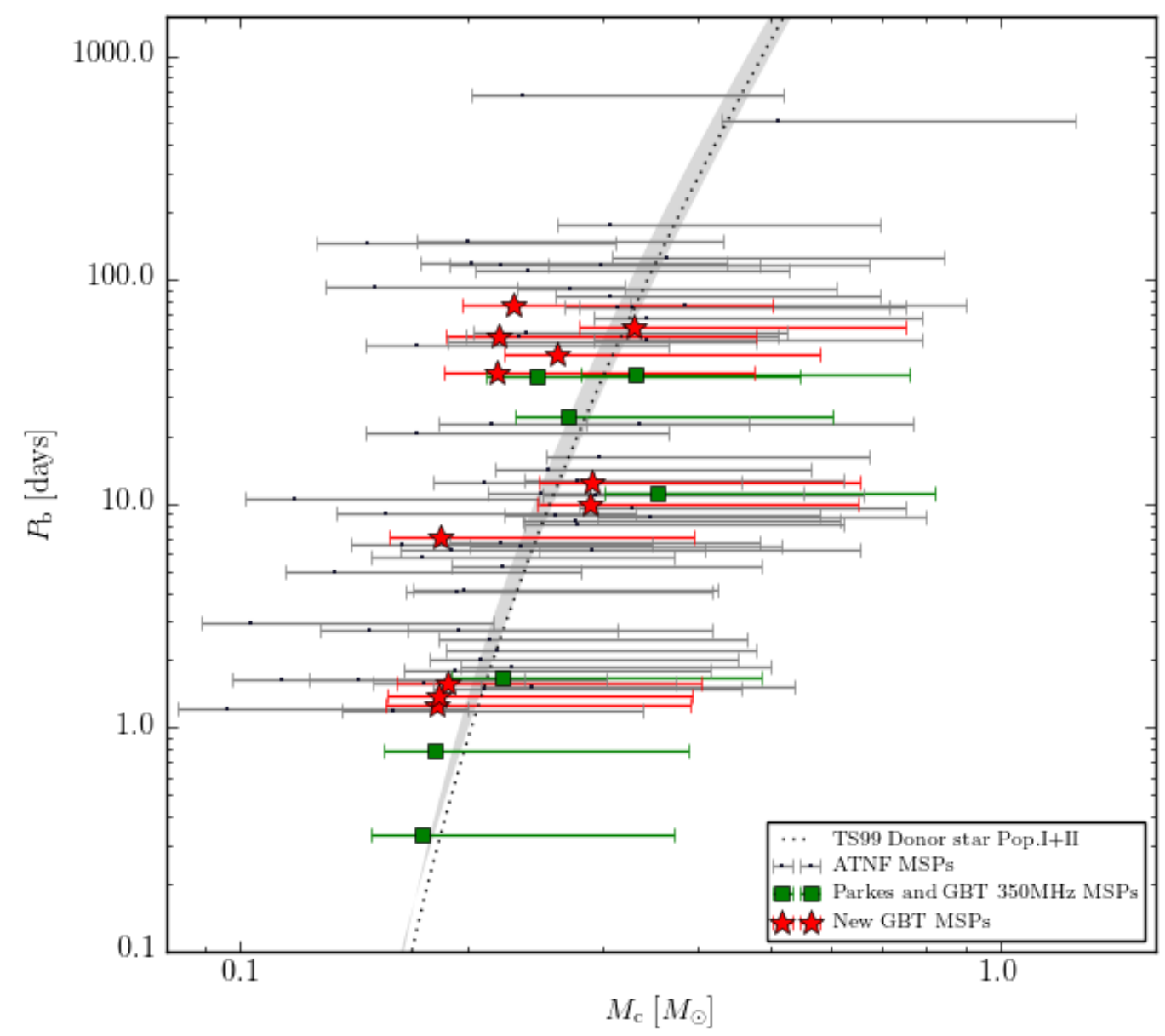

Fig. 2.6.-: A plot of TS99 relation with Galactic MSPs. The width of the relation is calculated from the Pop. I donor star relation (metallicities $Z=0.001$ ) and the Pop. II donor star (metallicities $Z=0.02$ ) relation. The dotted line shows the relation for Pop. I and Pop. II donor stars. Note that TS99 becomes uncertain for pulsars with $P_{b}<1$ day (dashed line). The data points show the median companion mass $\left(i=60^{\circ}\right.$ assuming $\mathrm{M}_{p}=1.48 M_{\odot}$ ), while the lower and upper limits correspond to the minimum companion mass with $i=90^{\circ}$ and a $90 \%$ confidence (assuming random orbital inclinations) maximum companion mass with $i=25.84^{\circ}$. 
with the TS99 relation is that orbital inclinations are distributed randomly, meaning that probability density is uniform or flat in $\cos i$. Stairs et al. (2005) and Smedley et al. (2014) examined this assumption by investigating binary MSPs with He-WD companions available at that time and found that the $\cos i$ corresponding to the $P_{b}-M c$ correlation is randomly distributed, as one might expect, if all pulsar masses are roughly $1.75 \pm 0.04 M_{\odot}$.

Using our much larger sample of binary MSPs we can also check the assumption of random orbital inclinations for binary MSPs. We can assume that TS99 is correct for He-WD MSP systems and then look at the resulting distribution of inferred inclinations. If pulsar orbits are randomly distributed and TS99 is correct, then $\sim 50 \%$ of the predicted median companion masses (i.e. those computed with $i=60^{\circ}$ ) should lie above the TS99 relation while the other $50 \%$ should lie below. However, we found that 56 out of 81 pulsars $(\sim 69 \%)$ fall below the TS99 relation (using Pop. I and Pop. II donor stars) when assuming all pulsar masses are $1.48 M_{\odot}$. The binomial probability for 56 or more out of 81 median masses to fall below the TS99 prediction, assuming an intrinsic probability of $50 \%$, indicates that this would occur by chance only $\sim 1.6 \times 10^{-4}$ of the time. The low binomial probability could suggest that TS99 is incorrect and/or that observed pulsar orbits are not randomly distributed. However, it is crucial to note that this number changes if we assume different pulsar masses (i.e. TS99 results in a flatter $\cos i$ distribution if we assume all pulsar masses are higher). This statement is supported by previous studies by Stairs et al. (2005) and Smedley et al. (2014). They examined the $\cos i$ distribution of binary MSPs with He-WD companions available at the time, and found (for $\cos i$ corresponding to the $P_{b}-M c$ relations $)$ that a large $\left(\sim 1.75 M_{\odot}\right)$ assumed pulsar mass yielded fairly flat $\cos i$ distributions. However, we know that pulsar masses are not all the same, and 
so assuming constant pulsar masses in these analyses is potentially problematic.

In order to test TS99's predictions with a more realistic distribution of pulsar masses, we performed a simple Monte Carlo analysis using the 81 pulsars described above. We ran 10,000 iterations where we randomly selected each pulsar mass from the Özel et al. (2012) normal distribution $\left(1.48 \pm 0.21 M_{\odot}\right)$ for recycled pulsars, such that every MSP system becomes consistent with TS99. Given TS99's predicted companion mass and the randomly drawn pulsar mass, the mass function determines the inclination of each system,

$$
f\left(m_{p}, m_{c}\right)=\frac{4 \pi^{2}}{G} \frac{(a \sin i)^{3}}{P_{b}^{2}}=\frac{\left(m_{c} \sin i\right)^{3}}{\left(m_{p}+m_{c}\right)^{2}},
$$

where $\mathrm{G}$ is the Newton's gravitational constant. For pulsars with known inclinations and companion masses, we used the known values: J0337+1715a (Ransom et al. 2014), J1713+0747, B1855+09, J1909-3744 (The NANOGrav Collaboration et al. 2015), and J0437-4715 (Verbiest et al. 2015, in prep).

Figure 2.7 shows the histogram of $\cos i$ from the statistical simulations, which indicates that the distribution is not uniform, but favors larger $\cos i$ (i.e. lower inclinations) in general. A KS-test comparing the simulated $\cos i$ distributions with a uniform distribution yields a median p-value of $1.1 \times 10^{-4}$, suggesting that we can reject a random inclination hypothesis at $\sim 0.01 \%$ confidence $(\sim 3.7 \sigma)$. Approximately $95 \%$ of the 10,000 simulation trials resulted in p-values smaller than 0.002 .

These results suggest that we may be preferentially observing MSPs with more face-on orbits. Perhaps MSP radio emission, which is certainly a wide fan beam given the number of MSPs with both radio and gamma-ray emission, is more concentrated near the spin axes of the pulsars (i.e. nearly aligned rotators assuming the magnetic field is aligned with the spin angular momentum), which we expect to be aligned with 
the orbital angular momentum due to accretion during the recycling process. Future emission studies, perhaps using radio polarization information and $\gamma$-ray emission modelling, may be able to investigate this aligned-emission hypothesis.

If our analysis is correct, and TS99 applies to these systems, we can make predictions about the properties of some of the MSPs. The analysis indicates that three MSPs should have low-mass neutron stars, with J1400-1438 having $m_{p}<1.28 M_{\odot}$, and both J1125-6014 and J1903-7051 having $m_{p}<1.16 M_{\odot}$. Similarly, the analysis suggests that five of the MSP systems (J1643-1224, J1748-3009, J1653-2054, $\mathrm{J} 2229+2643$, and $\mathrm{J} 1841+0130$ ) each have $i<30^{\circ}$ at $95 \%$ confidence. Interestingly, Fonseca et al. (in prep.) has come to a similar conclusion about J1643-1224 $\left(i<37^{\circ}\right)$ based on the lack of detection of Shapiro delay in that system. Also note that while the overall indications are for more face-on orbits, in general, there seems to be no evidence for pulsars with extremely face-on orbits (e.g. $i \lesssim 15^{\circ}$ ).

\subsection{Conclusion}

We report the discovery of twelve new MSPs from radio searches of Fermi LAT unassociated sources with the GBT. We have established phase-connected timing solutions for all twelve, and have detected $\gamma$-ray pulsations from each. We used a new MCMC gamma-ray timing technique to provide better measurements of $\dot{P}$ and proper motion due to the longer time span of the LAT $\gamma$-ray data. We also investigated 20 LAT sources near the Galactic plane in order to search for young pulsars, yet found no new pulsars there. The twelve new MSPs with timing solutions improve our knowledge of several aspects of the MSP population. Radio flux densities and $\gamma$-ray fluxes for $\gamma$-ray MSPs are currently almost completely uncorrelated, suggesting that we may yet find bright radio pulsars in faint $\gamma$-ray sources. We also investigated the 


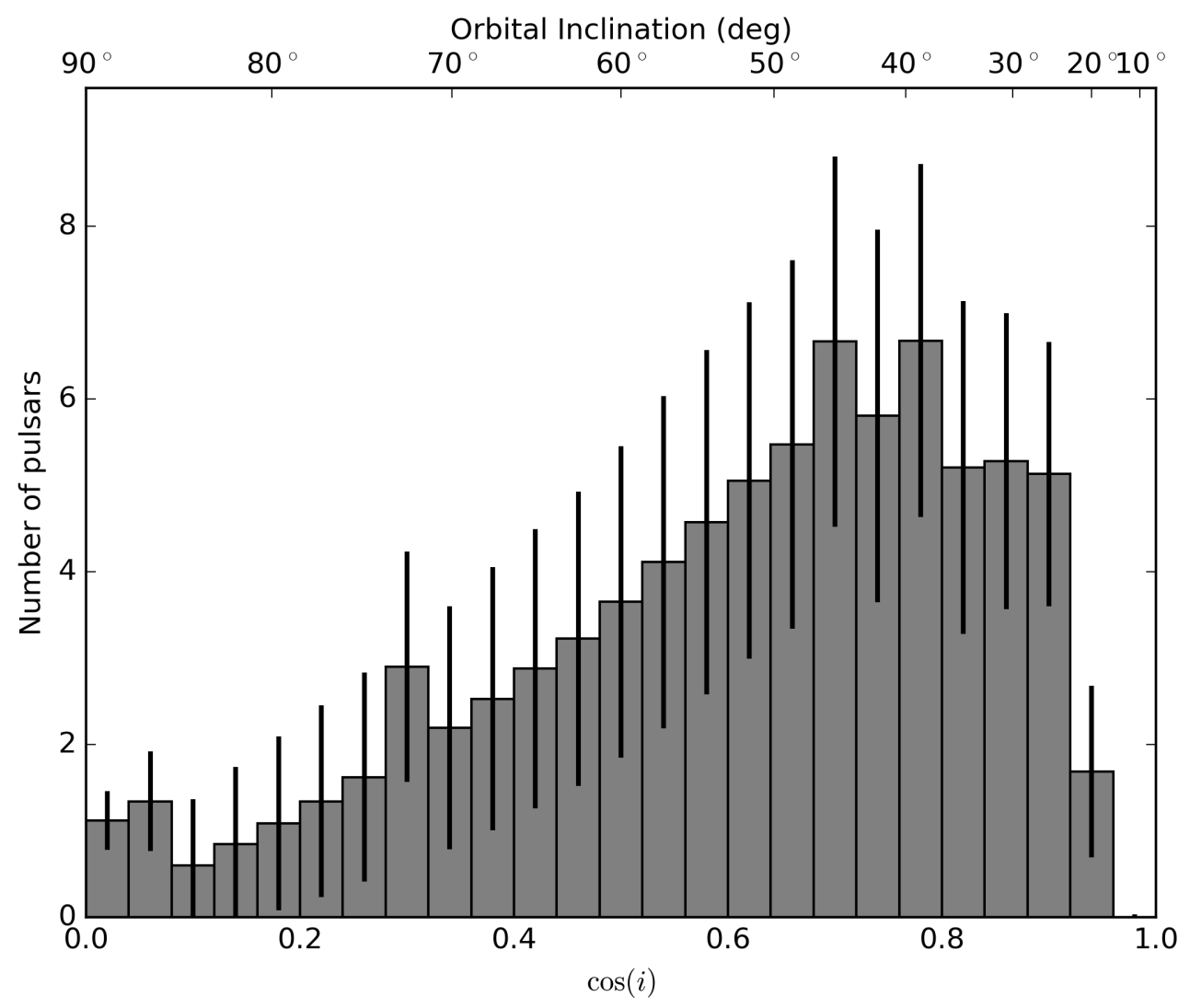

Fig. 2.7.- : For pulsar masses randomly selected from a normal distribution appropriate for recycled neutron stars $\left(1.48 \pm 0.21 M_{\odot}\right.$, based on Özel et al. 2012), we calculated corresponding companion masses which make the systems consistent with the TS99 relation, and thereby also determined the orbital inclinations of the systems. The histogram shows the distribution of $\cos i$ from 10,000 iterations of these simulations, with the vertical black bars being the $\pm 1-\sigma$ variation in each bin. The distribution is not flat as would be expected from random orbital inclinations (see $\S 2.5 .5)$. 
$P_{b}-M_{c}$ relation of pulsars with He-WD companions and found that the distribution of binary inclination angles may not be distributed randomly, but instead prefers lower inclinations (higher $\cos i$ ). 


\section{Chapter 3}

\section{Four New Pulsars in Tight Orbits}

\section{1 "Spider" Pulsars}

Millisecond pulsars (MSP) in compact binary systems are fascinating objects to study. At the present time, two types of compact binaries are known: the pulsar wind may be ablating away the companion or the pulsar may accrete mass from the companion, with the companion being gradually destroyed in both cases. Such cannibalistic behaviour suggested the arachnid nicknames for these types of systems: "Black Widows" (BWs) and "Redbacks" (RBs) respectively (it is a common practice to refer both to MSPs and to MSP+companion systems as BWs or RBs). BWs are rotationpowered MSPs in compact circular orbits with short orbital periods on the order of hours and very low mass companion $\left(\sim 0.01-0.05 M_{\odot}\right)$. RBs are similar systems, but with heavier non-degenerate companion of $\sim 0.1-0.4 M_{\odot}$. See Fig. 3.1 for an artist's impression of RBs and BWs.

BWs and RBs are thought to represent an important stage in pulsar evolution. In 1988 Fruchter et al. discovered the first known BW, B1957+20, and suggested that the isolated MSPs are formed from BWs after the pulsar wind completely evaporates 
the companion. The discovery of binary pulsars with extremely small companions, which are possibly ablated away by pulsar winds, (i.e. the pulsar planets Wolszczan \& Frail 1992) are indirect evidence for this scenario. However, Chen et al. (2013) demonstrated that it is difficult to produce the isolated MSPs from the BWs on a Hubble time scale. Thus, the evolutionary link between BWs and isolated MSPs remains unclear.

The discovery of the RB "missing link" system, PSR J1023+0038, brought evidence of back and forth transitions between Low Mass X-ray Binary (LMXB) states and radio pulsar states (e.g. Archibald et al. 2009; Papitto et al. 2013). RBs are therefore suggested to be the links between the accretion-powered X-ray pulsars and the rotation-powered radio pulsars.

The materials expelled from the companion often hinder the detection of pulsar emission. The eclipse mechanism is still unknown but is thought to be caused by either absorption or dispersive smearing of the radio waves (Thompson et al. 1994). While the absorption process, based on the interaction between the radio emission and the ejected material, decreases observed total flux density, the smearing process does not diminish the unpulsed flux density. It instead distorts its pulsed component. Smearing can be caused by the rapid DM variation in the ionized region of the companion which slightly increases the total line of sight electron column density. MSP detection is very sensitive to changes in the DM, so even a minimally altered DM could result in a non-detection of the pulsar.

Besides the main radio eclipse, happening around the inferior conjunction, the unbound plasma can also cause short irregular eclipses at random orbital phases. This is a distinctive phenomenon of BW and RB systems. Sometimes, immediately before and after the eclipse (for both regular and irregular eclipses), an increase in 
the DM is observed, indicating that the line of sight passes directly through the intrabinary plasma.

For the BW and RB systems, an intrabinary shock may have formed between the pulsar and the companion at the location where pulsar-wind pressure balances with the wind pressure from the companion. The so-called pulsar-wind shock (pulsar wind nebulae) can be the source of high-energy (X-ray or $\gamma$-ray) emission modulated at the orbital period (e.g. Dubus 2015; Huang et al. 2012) in addition to pulsations from the pulsar itself.

Prior to the launch of Fermi, most BWs and RBs known resided in globular clusters (e.g. Ransom et al. 2005; Hessels et al. 2007). The Galactic BWs and RBs were the rare systems; only one RB (Chakrabarty \& Morgan 1998) and three BWs were known (Johnston et al. 1992; Fruchter et al. 1988; Lundgren et al. 1995; Stappers et al. 1996, respectively). The radio searches of Fermi-LAT unassociated sources have so far revealed 15 new BWs and 9 new RBs, which now bring the total number of these systems to 28 (and counting) (Grenier \& Harding 2015). Despite the dramatic seven-fold increase, the number of BW and RB systems known is still not large and there is plenty of room for new discoveries within the sample of unidentified Fermi sources. As more RB and BW systems are discovered, we will better understand the stages of pulsar evolution, explore the intricate interaction between pulsar emission and the intrabinary plasma and, in general, learn more about this exotic part of the pulsar population.

\subsection{Observation and data analysis}

The goal of GBT's AGLST14A_480 project (PI Sanpa-Arsa) was to search for new radio MSPs within the internal Fermi LAT 4-year pulsar-like source catalog (now 
3FGL). The observations were conducted during year 2014, in the same manner as described in Chapter 2. Thus, here I will give only a brief summary of the observing setup.

In total, 68 sources were observed. Depending on the source location and the size of the $95 \%$ position error box, either the $350 \mathrm{MHz}$ - or $820 \mathrm{MHz}$-centered GBT receiver was used. The sources with Galactic latitude $(|b|)$ between $2^{\circ}$ and $5^{\circ}$ and with position error boxes smaller than $16^{\prime}$ can be observed with a single $820-\mathrm{MHz}$ pointing (16' GBT beam FWHM at $820 \mathrm{MHz})$. The sources above Galactic plane $\left(|b|>5^{\circ}\right)$ and with error box smaller than $36^{\prime}$ were observed with the GBT's 350-MHz receiver (FWHM of $36^{\prime}$ ). Using lower frequencies allowed us to explore sources with less precise positions, but this method cannot be used for the low-latitude candidates because of the sharp rise of the sky temperature in the Galactic plane at lower observing frequencies $\left(T_{\text {gal }} \sim \nu^{-2.55}\right.$, Lawson et al. 1987).

The data were recorded with the Green Bank Ultimate Pulsar Processing Instrument (GUPPI) backend in search mode (DuPlain et al. 2008) and then processed offline. The processing steps included RFI removal, dedispersion, acceleration and periodic pulsar searches (see Chapter 2 for details). In addition, we performed singlepulse search on each source.

Upon discovery, the follow-up observations were scheduled in order to obtain TOAs and refine pulsar ephemerides, using the GBT at $820 \mathrm{MHz}$ and GUPPI. For PSR J1513-2550, we acquired additional extended timing observations with the Nançay telescope at $1.48 \mathrm{GHz}$ using the NUPPI backend with 1024 channels over $512 \mathrm{MHz}$ bandwidth. Once timing solution had been firmly established, we used it to search for the $\gamma$-ray-pulsations from the same source.

Throughout this chapter, we count the orbital phase clockwise from the ascending 
node. This implies that orbital phase is 0.25 when the companion is between the pulsar and the Earth (inferior conjunction). This is the phase where eclipses are expected to happen.

\subsection{Results}

\subsubsection{Pulsar timing}

We were able to phase-connect the four new MSPs with the follow-up observations spanning over 2.3 years using the GBT and the Nançay telescope. We found that PSR J1622-0315 and J2017-1614 are likely to exhibit $\gamma$-ray pulsation and have optical counterpart. J1622-0315 and J2155+5448 shows notable flux variation in many observation. We also were able to measure the proper motion of J1622-0315. The residual plot of the three MSPs are shown in Fig.3.2.

Three MSPs (PSRs J1513-2550, J2017-1614 and J2115+5448) were best-fit with the ELL1 binary model (see Table5.8), which indicates a circular orbit (the eccentricity is negligible). They have not had proper motion measurements from pulsar timing yet; therefore a purely kinematic contribution to the observed period derivative $\left(\dot{P}_{\text {obs }}\right)$, known as the Shklovskii effect $\left(\dot{P}_{\text {Shk }}\right)^{1}$, cannot be computed yet. This means that the physical parameters $\dot{E}$ and $B$ of the pulsar, which depend on $\dot{P}$, are currently upper limits.

PSR J1622-0315, a new RB, on the other hand, is best-fit with the BTX binary model (see Table 5.9), which allows multiple derivatives of the orbital frequency to be included in the timing model. The proper motion is measured with sufficient significance and consequently yields the $\dot{P}_{\text {Shk }}$ of $17 \times 10^{-21}$. This value is, however,

\footnotetext{
${ }^{1} \dot{P}_{\text {Shk }}=\mu^{2} P d / c$, where $\mu$ is the pulsar proper motion, $P$ is the spin period, $d$ is the distance to the system, and $c$ is the speed of light
} 
higher than the $\dot{P}_{\text {obs }}$ of $11.6 \times 10^{-21}$. This implies that the DM-derived distance $(d)$ is likely overestimated resulting in the overestimation of $\dot{P}_{\text {Shk }}$.

\subsubsection{The new MSPs}

\section{PSR J1513-2550}

PSR J1513-2550 is a millisecond pulsar with spin period of $2.12 \mathrm{~ms}$, DM of $47.12 \mathrm{pc} \mathrm{cm}^{-3}$, orbital period of 4.3 hours and minimum companion mass of $0.02 M_{\odot}$. It is in a tight orbit with very low companion mass $\left(\geq 0.02 M_{\odot}\right)$ and exhibits eclipses in some observations. J1513-2550 is therefore considered a black widow pulsar. See Fig. 3.3 for the discovery plot.

PSR J1513-2550 is a very bright MSP with a mean flux density at $820 \mathrm{MHz}$ of $\sim 2$ mJy. In general, bright MSPs with narrow pulse profiles like PSR J1513-2550 yield high quality TOAs and, therefore, small timing residuals of order $\mu s$. They are excellent candidates for pulsar timing arrays (PTAs) which aim to detect gravitational waves; for instance, the North American Nanohertz Observatory for Gravitational Waves (NANOGrav), the European Pulsar Timing Array (EPTA), and the Parkes Pulsar Timing Array (PPTA) (see Hobbs et al. (2010) for a review). Normally, BWs and RBs exhibits orbital period variations over time; therefore orbital frequency derivatives are usually required for BWs and RBs timing. Adding more parameters, however, reduces sensitivity to gravitational waves, so including BWs and RBs into PTAs is potentially troublesome. However, the lost of phase connection due to orbital period variability only happens in RBs. The orbital period change of BWs is slow enough that we can still keep them phase connected. In addition, Bochenek et al. (2015) showed that for fast, strong, narrow-pulsed and non-eclipsing BWs, fitting for the orbital period derivatives reduces less than $5 \%$ of the gravitational wave sensi- 
tivity. They recommended using these suitable BWs in the PTAs. Unfortunately, J1513-2550 shows strong and irregular radio eclipses; thus, it is likely not suitable for the PTAs.

\section{PSR J1622-0315}

PSR J1622-0315 has a spin period of $3.86 \mathrm{~ms}$, DM of $21.4 \mathrm{pccm}^{-3}$, orbital period of 3.9 hours, minimum companion mass of $0.1 M_{\odot}$, and shows strong and irregular eclipses. PSR J1622-0315 can therefore be classified as a redback system. See Fig.3.4 for the discovery plot. The orbital frequency derivative is significantly measured at $1.57(7) \times 10^{-19} \mathrm{~Hz} \mathrm{~s}^{-1}$ which indicates the orbital period changes with time due to classical effects from the main sequence companion star. This pulsar eclipses for nearly half of the orbit and when it is "on", it displays significant flux density variability due to diffractive scintillation in most observations. It additionally exhibits smaller short-duration eclipses during some observing sessions (see Fig. 3.13).

With the precise pulsar ephemeris, we folded the Fermi LAT photons and found a plausible $\gamma$-ray pulsation at $6.9 \sigma$. However, we still need to improve the ephemeris in order to assert the pulsation. We also identified a star from the Sloan Digitized Sky Survey (SDSS) at the position of the pulsar as the optical counterpart. In addition, we fold data from R-band images obtained at the Hiltner 2.4-m Telescope of the Michigan-Dartmouth-MIT (MDM) Observatory on June 2016 and found that the photometric light curve shows sinusoidal variations (Jules Halpern, private communication). The variation is consistent with ellipsoidal distortion from a companion that is overflowing its Roche lobe. Such ellipsoidal distorion leads to two maxima and two minima per orbit on the light curve (see Fig. 3.5). The maxima occur equally at phases 0.0 and 0.5 when the tidally distortion of a companion is largest, whereas the 
minima occur unequally at phases 0.25 and 0.75 . The inequality is due to the fact that the inner Lagrangian point (L1) of a companion viewing at phase 0.75 encounters larger effect of limb and gravity darkening; therefore flux at phase 0.75 is lower in magnitude ( $\mathrm{Li}$ et al. 2014). The optical variation validates the astrometric position and orbital frequency derivative measurement from pulsar timing.

\section{PSR J2017-1614}

PSR J2017-1614 is another black widow pulsar with spin period of $2.31 \mathrm{~ms}$, DM of $25.44 \mathrm{pc} \mathrm{cm}^{-3}$, orbital period of 2.3 hours, and minimum companion mass of $0.03 M_{\odot}$. See Fig. 3.6 for the discovery plot. With a relatively weak radio signal and broad pulse profile, PSR J2017-1614 has quite poor timing precision (Fig. 3.2), with timing RMS of $17 \mu \mathrm{s}$.

By folding LAT $\gamma$-ray photons with the timing ephemeris of PSR J2017-1614, $\gamma$-ray pulsations with a significance of $5.7 \sigma$ were found (see Fig. 3.7). The detection is relatively weak but may be improved with an updated ephemeris. We also found a variation in the optical light curve of the companion star from data from the $2.4-\mathrm{m}$ at MDC. The modulation is dominated by a process of heating a companion by the pulsar wind which contributes most at phase 0.75 (see Fig. 3.8; Jules Halpern, private communication). In general, light curves of BWs are most affected by heating from the MSP wind due to their very compact orbits.

\section{PSR J2115+5448}

PSR J2115+5448 is a black widow pulsar with spin period of $2.6 \mathrm{~ms}$, DM of $77.41 \mathrm{pc}$ $\mathrm{cm}^{-3}$ orbital period of 3.2 hours, and minimum companion mass of $0.02 M_{\odot}$. See the discovery plot at Fig. 3.9. 
PSR J2115+5448 exhibits short-duration eclipses during many observations and has an orbital period derivative $\left(\dot{P}_{B}=6 \times 10^{-11} \mathrm{~s} \mathrm{~s}^{-1}\right)$, suggesting a modulation in orbital period. It has a high spin-down luminosity $(\dot{E})$ of $1.65 \times 10^{35} \mathrm{erg} \mathrm{s}^{-1}$, placing it in a group of few Fermi MSPs with the spin-down luminosity more than $10^{35} \mathrm{erg} \mathrm{s}^{-1}$. However, a contribution in $\dot{P}_{\text {obs }}$ from the Shklovskii effect has not been measured yet which results in an overestimation in $\dot{P}$ and therefore an overestimation in $\dot{E}$ $\left(\dot{E} \propto \dot{P} / P^{3}\right)$.

\subsubsection{Single-pulse search}

This section is contributed by Anna Bilous

For all four pulsars the single-pulse search was run on the discovery session. This search did not yield any positive results (see Figures 3.10 and 3.11): no excess of single-pulse candidates were detected at the respective DMs of all four pulsars. Search sensitivity was calculated using Eq. 2.3. The $S_{\text {thr }} \times \sqrt{n_{\text {box }}}$ was 0.4 Jy for $820 \mathrm{MHz}$ (for the threshold $\mathrm{S} / \mathrm{N}$ of 5 ).

The energies of single pulses from the non-recycled pulsars usually have normal or log-normal distribution (Burke-Spolaor et al. 2012). The analogous distributions for MSPs (let alone RB/BW systems) are not very well known, however, some of the MSPs are known to emit, in addition to normal pulses, a distinct class of pulses called Giant Pulses (GPs). GPs are bright narrow pulses with a steep power-law energy distribution. According to Knight (2006), for the established GP emitters, which are a very small segment of the MSP population, the probability of detecting a GP with the energy 20 times exceeding the energy of the average profile ${ }^{2}$ varies between $10^{-4}(\mathrm{~B} 1937+21)$ to $10^{-7}$ per pulsar spin period.

\footnotetext{
${ }^{2}$ Or, equivalently, with $S_{\text {sps }} / S>20$.
} 
Taking into account the $S_{\mathrm{sps}} / S$ from Table3.1 and the corresponding lengths of sessions measured in pulsar spin periods, it can be concluded that our pulsars do not emit GPs much brighter than those of PSR B1937+21. However, the sensitivity and time span of the observations do not allow us to rule the GP-like emission completely.

Two additional notes should be made. Firstly, the average flux of RB/BW systems is known to vary within an order of magnitude. Thus, other, future single-pulse searches can be more fruitful. Secondly, the width of GPs can be smaller than our time resolution of $64 \mu \mathrm{s}\left(n_{\text {box }}=1\right)$, which effectively reduces the sensitivity.

\subsection{Discussion}

\subsubsection{Flux variations}

All of the new MSPs exhibit eclipses and/or flux variations. PSR J1513-2550 and PSR J2017-1614 show eclipses in some sessions but show no sign of flux variability in others (see Fig. 3.12 and 3.14). PSR J2115+5448 and PSR J1622-0315, however, both exhibit regular and irregular eclipses, and flux variability in different observations (see Figures 3.15 and 3.13). In general, flux variation of a pulsar is a result of significant scattering effects from the interstellar medium (ISM) along the line of sight. The variation in flux of BW and RB, however, can either caused by the scattering effect from the ISM or ejected particles from a companion, or both. Studying

\begin{tabular}{cccc} 
Pulsar & $S_{\mathrm{sps}} / S \times \sqrt{n_{\mathrm{box}}}$ & Length of session, s & Length of session, $P$ \\
\hline J1513-2550 & 5088 & 1966 & $9.4 \times 10^{5}$ \\
J1622-0315 & 6467 & 2580 & $6.8 \times 10^{5}$ \\
J2017-1614 & 35617 & 2950 & $1.3 \times 10^{6}$ \\
J2115+5448 & 7877 & 2950 & $1.1 \times 10^{6}$
\end{tabular}

Table 3.1:: Sensitivity to single pulse flux and observing session durations. 
the flux variation in BWs and RBs, thus, can probe properties of the ISM and/or particles expelling from the companion.

\subsubsection{Optical, X-ray and $\gamma$-ray counterparts}

Multi-wavelength studies of BWs and RBs bring much additional information about the MSPs and their companions in these fascinating binary systems. The shape and peak of the optical light curve from photometry of the binaries reveals information about the companion as well as an interaction between the companion and the pulsar. The sinusoidal shape of the light curve indicates the modulation of the near-Roche lope filling companion (as seen in PSR J1622-0315). The light curve peak at orbital phase of 0.75 (superior conjunction) suggests irradiation from the pulsar which means the pulsar is possibly ablating the companion. Furthermore, the double peaks at phase zero and 0.5 indicate tidal interaction between the pulsar and the companion. Colors from optical photometry additionally reveal physical properties of the companion namely radius and temperature.

By monitoring the BWs or RBs at high energies (X-ray and $\gamma$-ray), one may detect the orbital modulation in X-ray or $\gamma$-ray which could be the result of intrabinary shocks where the pulsar-wind pressure balances the wind pressure from the companion star (e.g. Archibald et al. (2010) and Xing \& Wang (2015)). Additionally, drastic increases in the $\gamma$-ray flux and the disappearance of radio pulsations in RBs could signal the transition from rotation-powered to accretion-powered pulsar states (Stappers et al. 2014). Observing RBs with optically bright companions can help

constrain the mass function of the companion and the pulsar (Bellm et al. 2016). Furthermore by regularly monitoring RBs in multiple wavebands, we may be able to find a new transitioning pulsar. This is because the transition between the accretion- 
power stage (LMXBs: X-ray loud, radio quiet) to the rotation-power stage (binary pulsar: X-ray quiet, radio loud) can take place within the relatively short time scale of years or even months (e.g. Archibald et al. 2015; Benvenuto et al. 2015)

\subsection{Conclusion}

In this chapter, we investigate the properties and counterparts of four new MSPs in compact orbits with low mass companions, known as black widows (BWs) and redbacks (RBs), discovered with the GBT in Fermi unassociated sources. PSR J1513-2550, J2017-1614 and J2115+5448 are BWs and PSR J1622-0315 is a RB. All four MSPs are eclipsing which is one of the signature behaviours of these systems. PSR J1622-0315 and PSR J2155+5448 exhibit "irregular" short-time eclipses at a random orbital phase. The short-duration eclipses are the result of material ejected from the companion which result in flux density variability. From the follow-up observations at the GBT and the Nançay telescope, we obtained phase-connected timing solutions for all of the pulsars. From pulsar timing, we were able to measure the orbital period derivative of PSR J1622-0315 and J2115+5448 and the proper motion of PSR J1622-0315. With the precise timing ephemerides, we folded Fermi LAT photons and found that PSR J1622-0315 and J2017-1614 have likely significant $\gamma$-ray pulsations. The optical light curve from PSR J1622-0315 is sinusoidal at half the orbital period, indicating that the companion is filling its Roche lobe. We also performed single-pulse searches on all pulsars and found no significant individual pulses.

The BWs and RBs are thought to be the missing links in pulsar evolution. Therefore monitoring these systems in multiple wavelength bands will illuminate other properties of these fascinating systems. Such observations will allow us to glimpse the eclipse mechanism(s), the transition from LMXBs to rotation-powered pulsars, 
and the formation of isolated MSPs. 


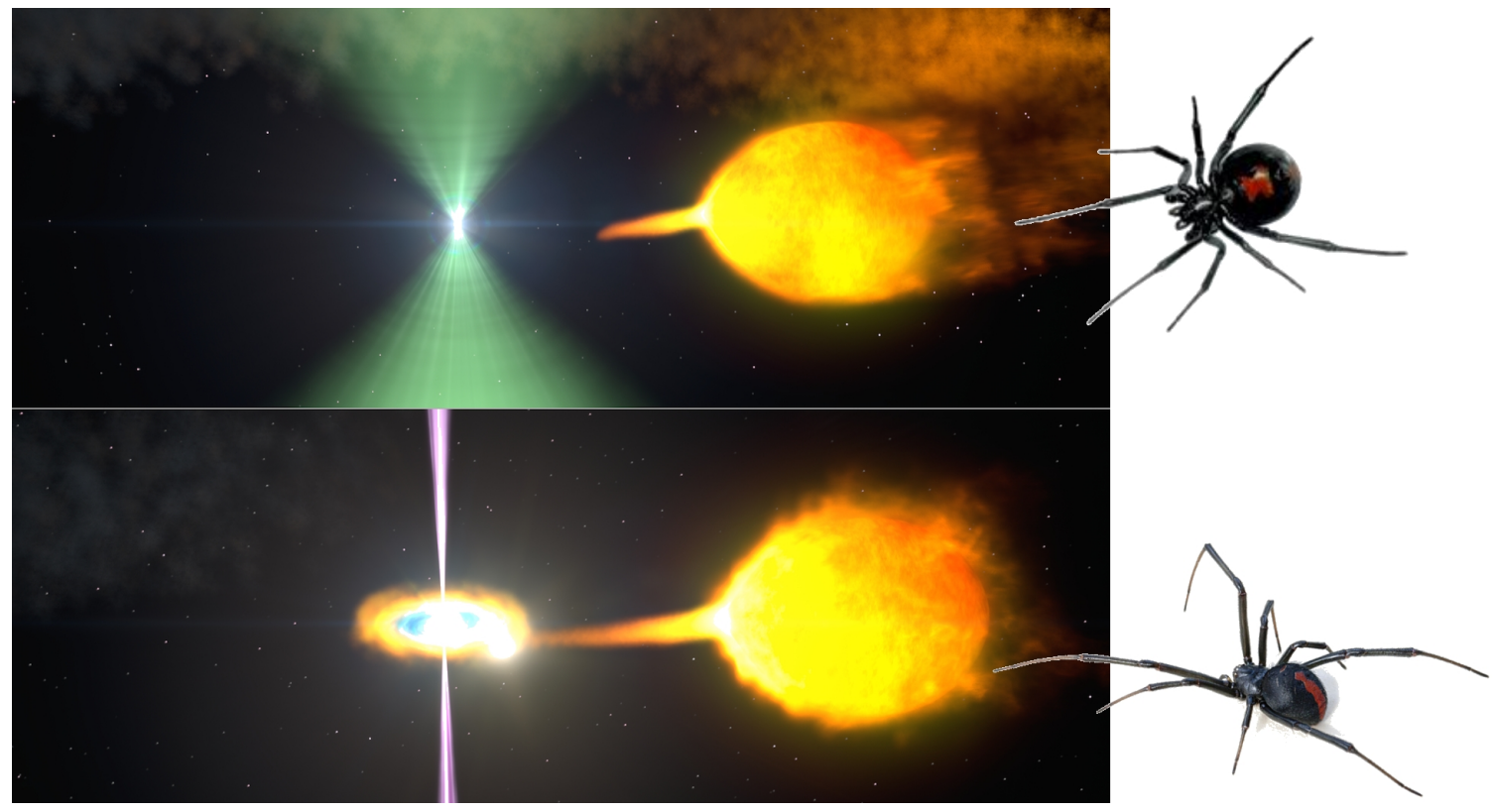

Fig. 3.1.- : Artist's impression of a redback (top) and a black widow binary systems together with the photgraphs of their arachnid namesakes. (Image credits: NASA Goddard's Scientific Visualization Studio, Wikipedia) 


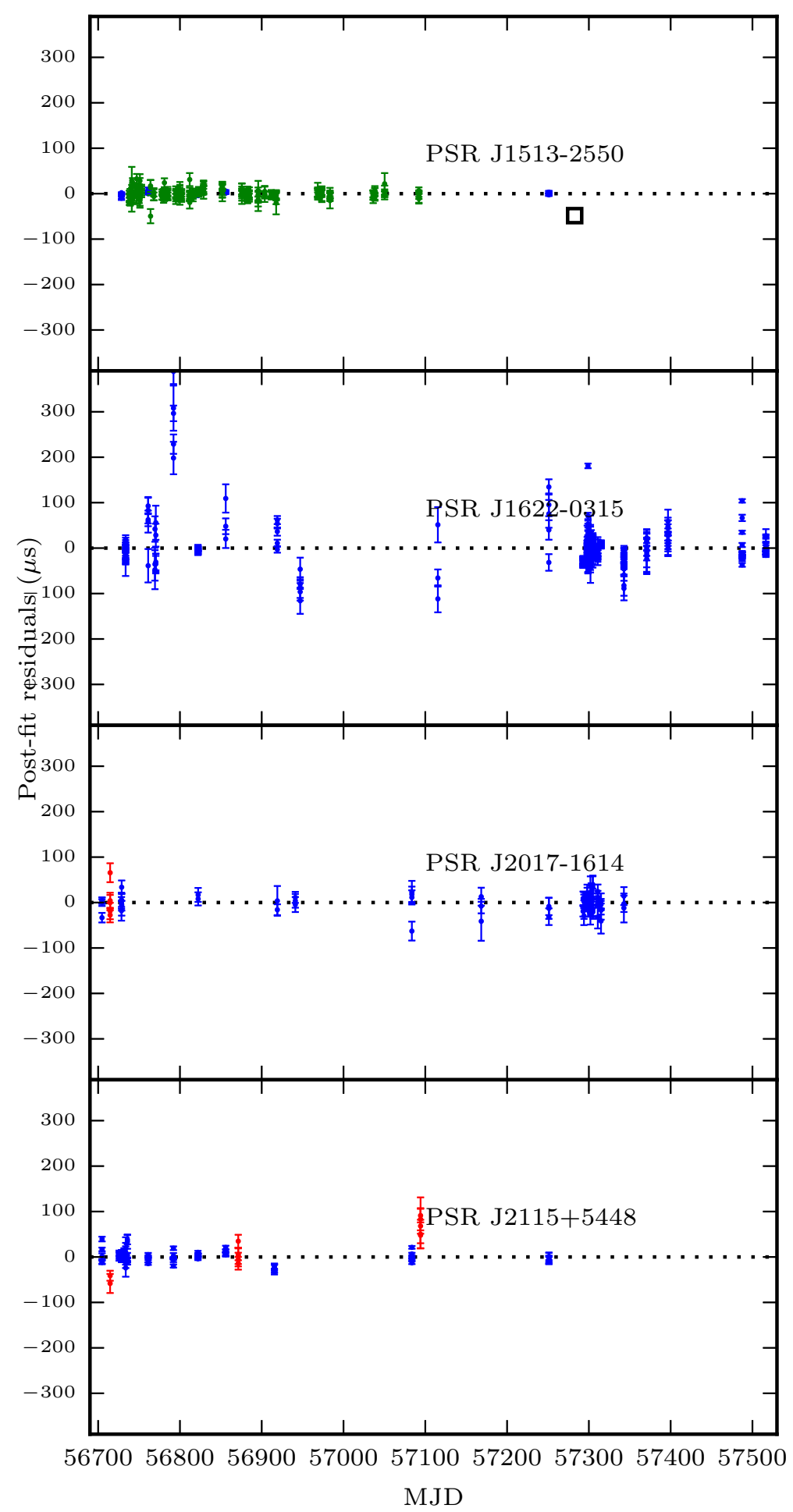

Fig. 3.2.-: The residual plots of PSR J1513-2550, PSR 1622-0315, PSR J2017-1614 and PSR J2115+5448 as a function of observing time in MJD and the residual time in $\mu s$. The red, blue and green dots are the observation at $350 \mathrm{MHz}$, $820 \mathrm{MHz}$ and $1400 \mathrm{MHz}$ respectively. 


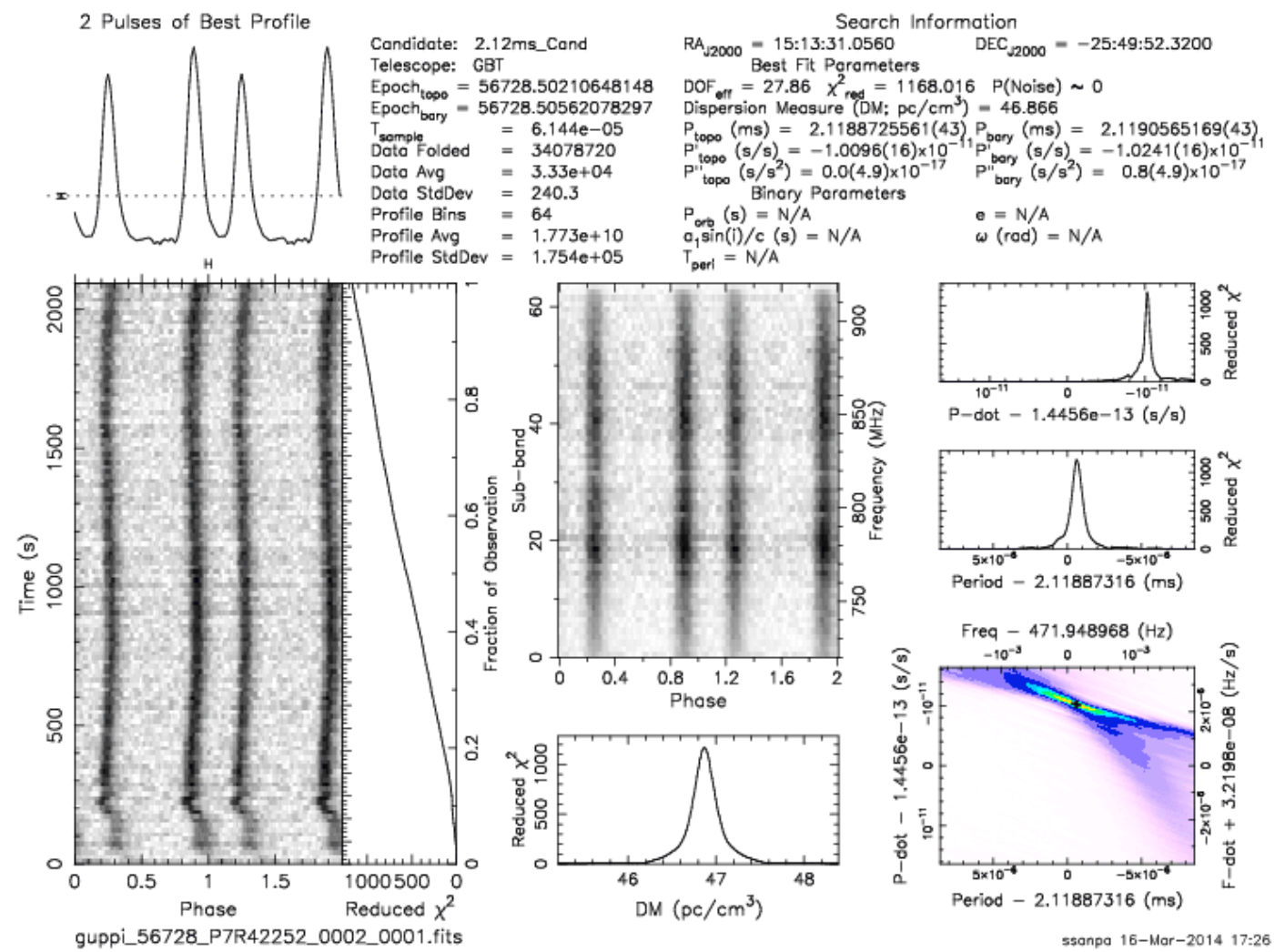

(a)

Fig. 3.3.-: The discovery plots of PSR J1513-2550. The pulsar signal is very bright, resulting in the prominent peak in DM- $P-\dot{P}$ parameter space. In this 30 min observation, the period shows almost no acceleration-induced variation, however, there is some evidence of the eclipse egress or confusion due to gases from a companion at the start of the observing session. 


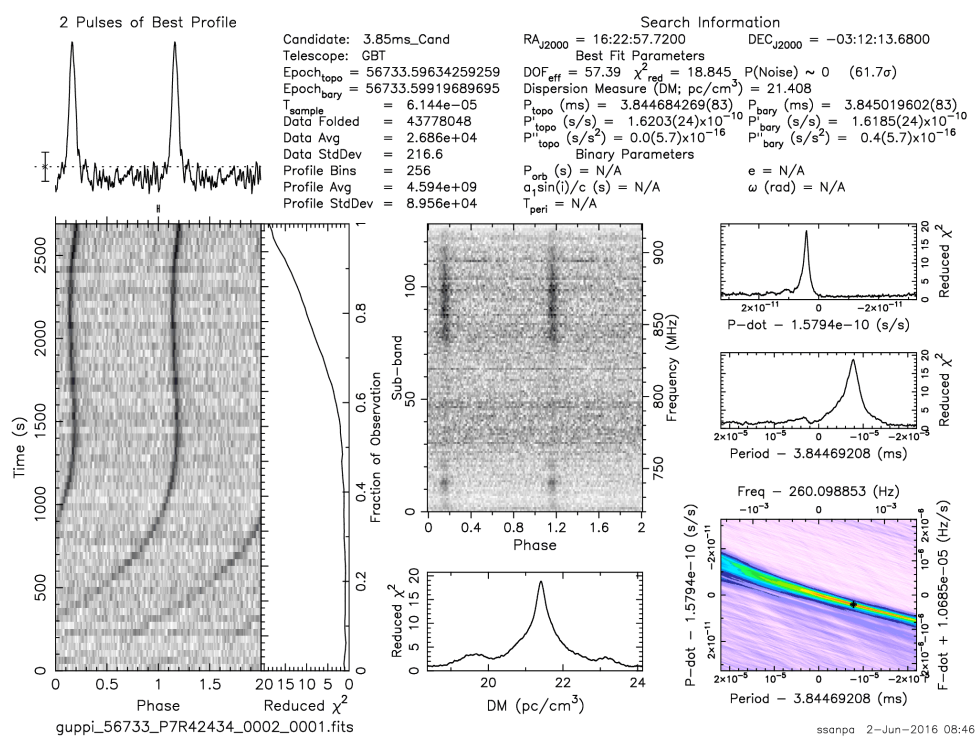

(a)

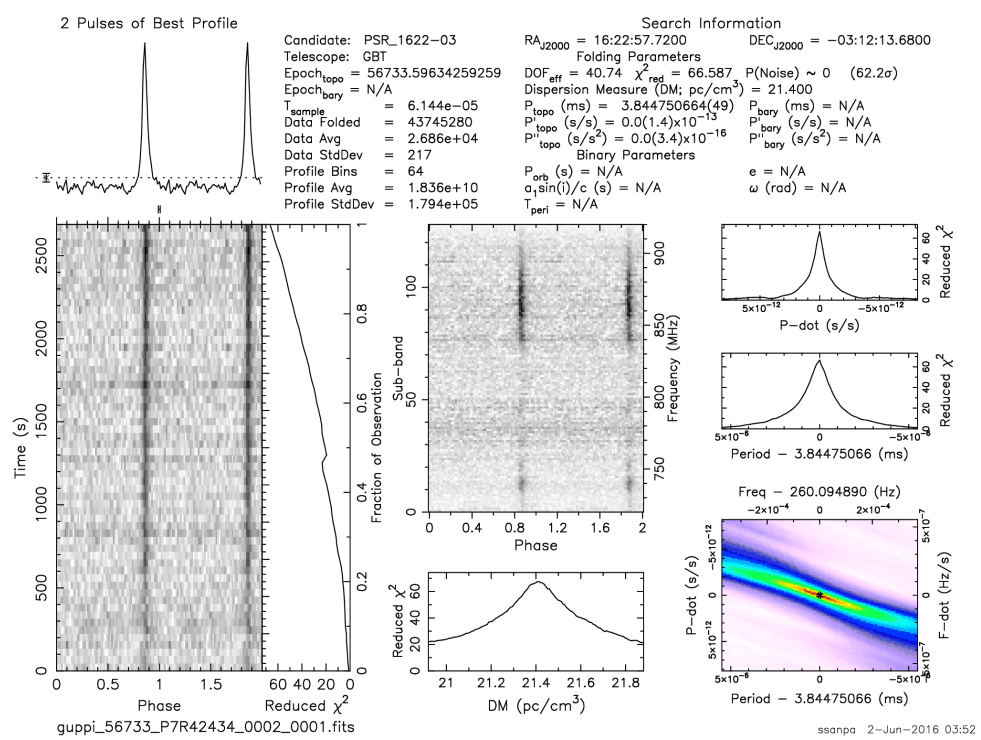

(b)

Fig. 3.4.- : The discovery plot of PSR J1622-0315 is shown on panel 3.4a. The curvature of the pulsar-like signal on the waterfall plot indicates large acceleration of the star. This curvature can be corrected by folding the raw data with an improved pulsar ephemeris (panel 3.4b). The cloud at the beginning of the session could be a result from eclipse or scintillation. 


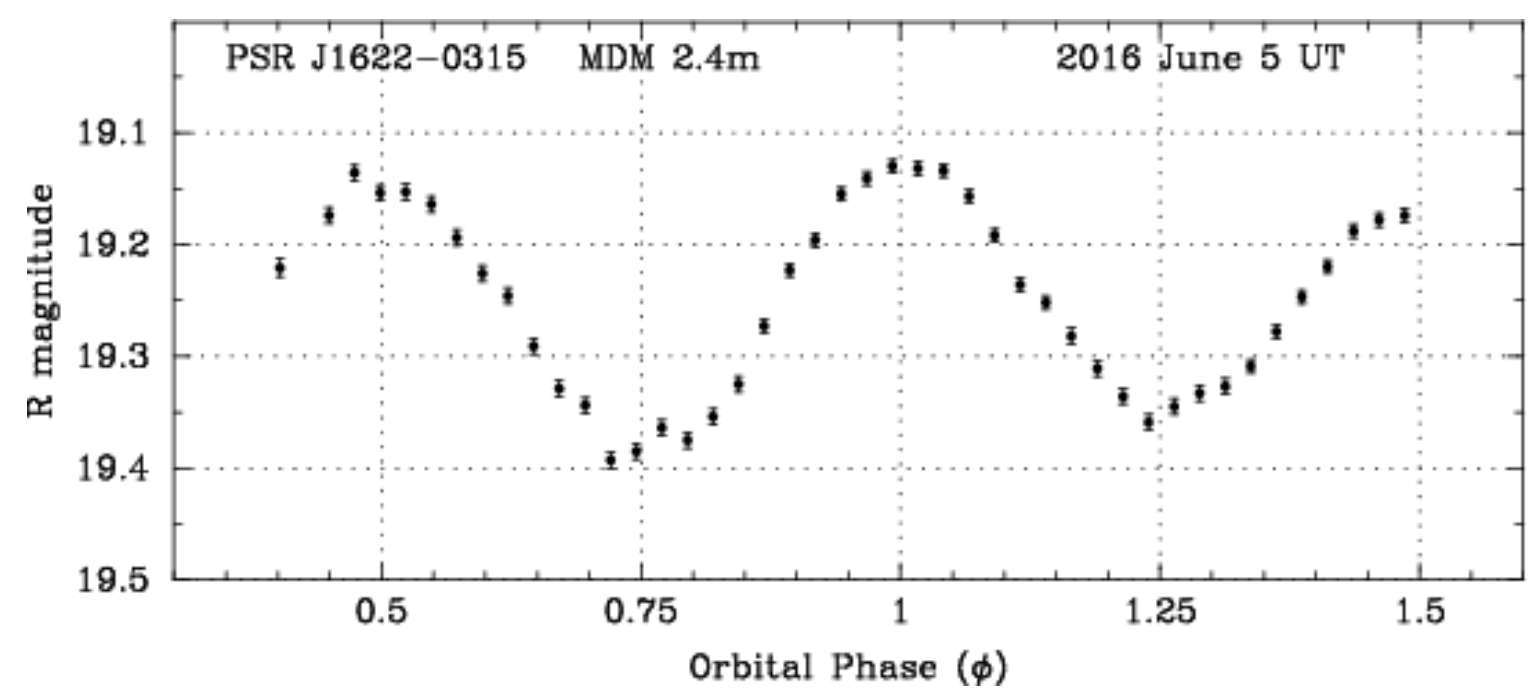

Fig. 3.5.- : Optical light curve of PSR J1622+0315 taken with the 2.4-m Telescope of the MDM Observatory on 2016 June 5 shows an sinusoidal modulation in magnitude which is consonant with ellipsoidal distortion from a companion filling its Roche lobe. Image credit: Jules Halpern 


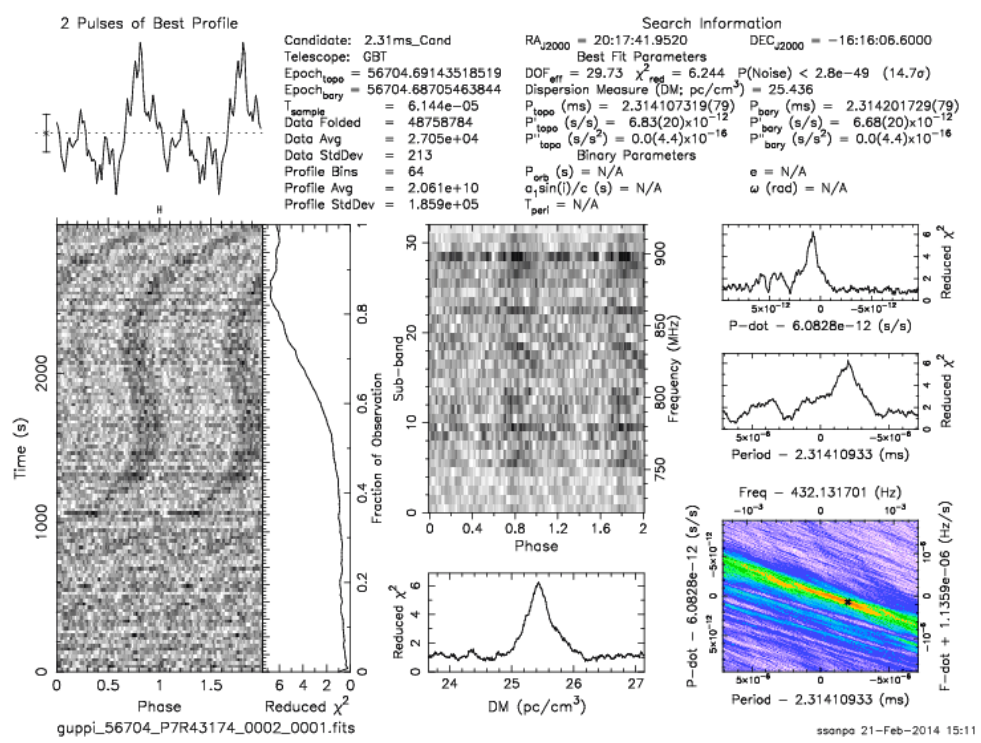

(a)

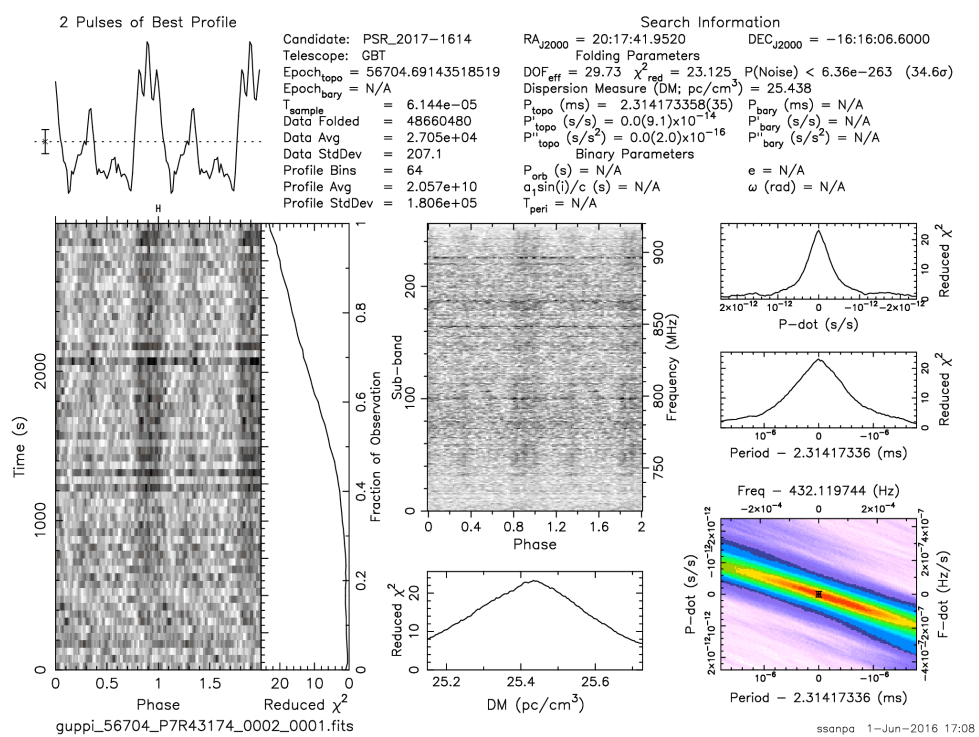

(b)

Fig. 3.6.- : The discovery plot of PSR J2017-1614. The acceleration from the pulsar orbital motion shows as a curvature in the waterfall plot on the panel 3.6a which is corrected by folding data with an improved ephemeris from the timing solution as shown in panel 3.6b. The beginning of the observation shows evidence of an eclipse. 


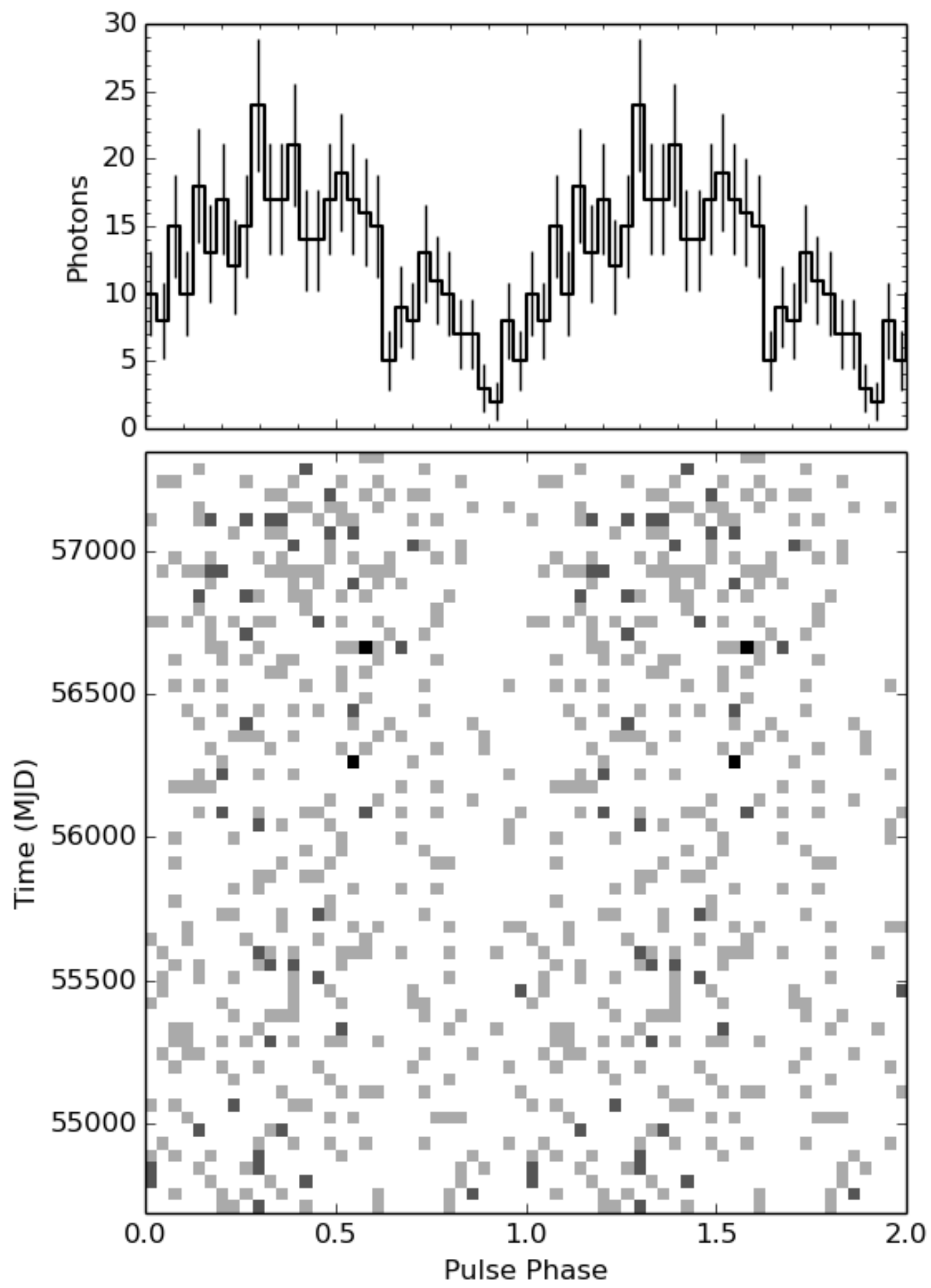

Fig. 3.7.-: The phaseogram that shows a weak $\gamma$-ray pulsation of PSR J2017-1614. The detection threshold is $5.7 \sigma$ with a radius $<0.5$ degree and energy $>900 \mathrm{Mev}$ cut-off. Image credit: Paul Ray 


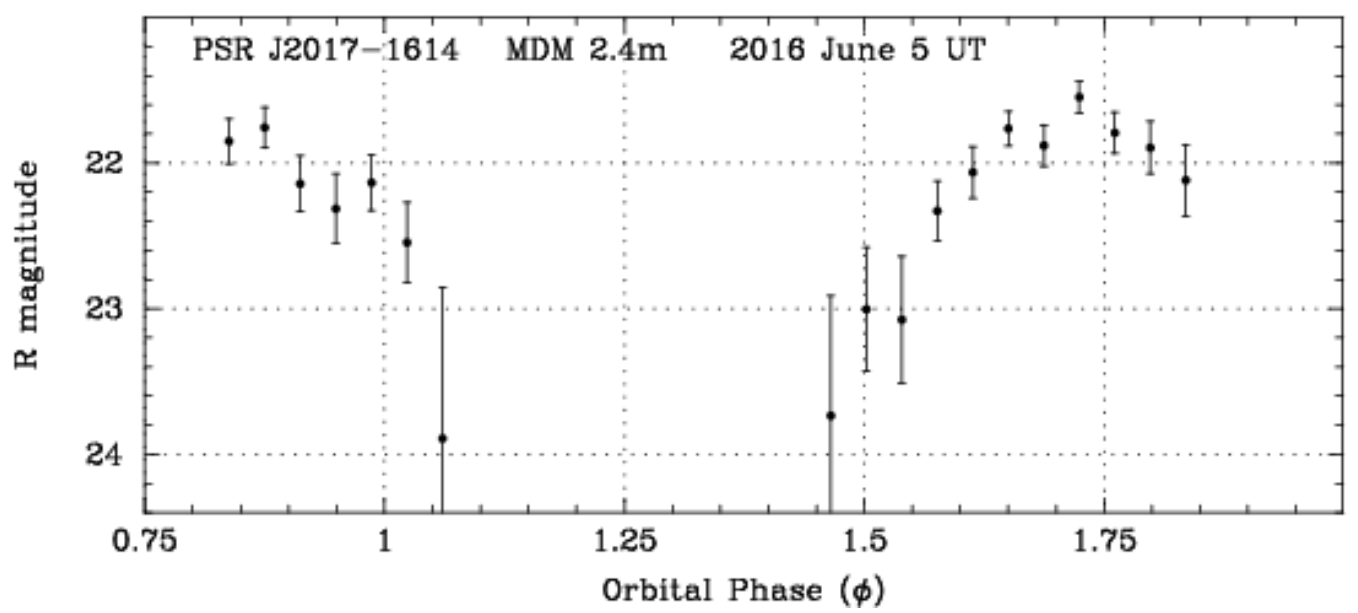

Fig. 3.8.-: Optical light curve of PSR J2017-1614 taken with the 2.4-m Telescope of the MDM Observatory on 2016 June 5. Even though the light curve only displays a partial orbit, it shows maximum brightness at phase 0.75 , when heating from the pulsar should be maximized. Image credits: Jules Halpern 

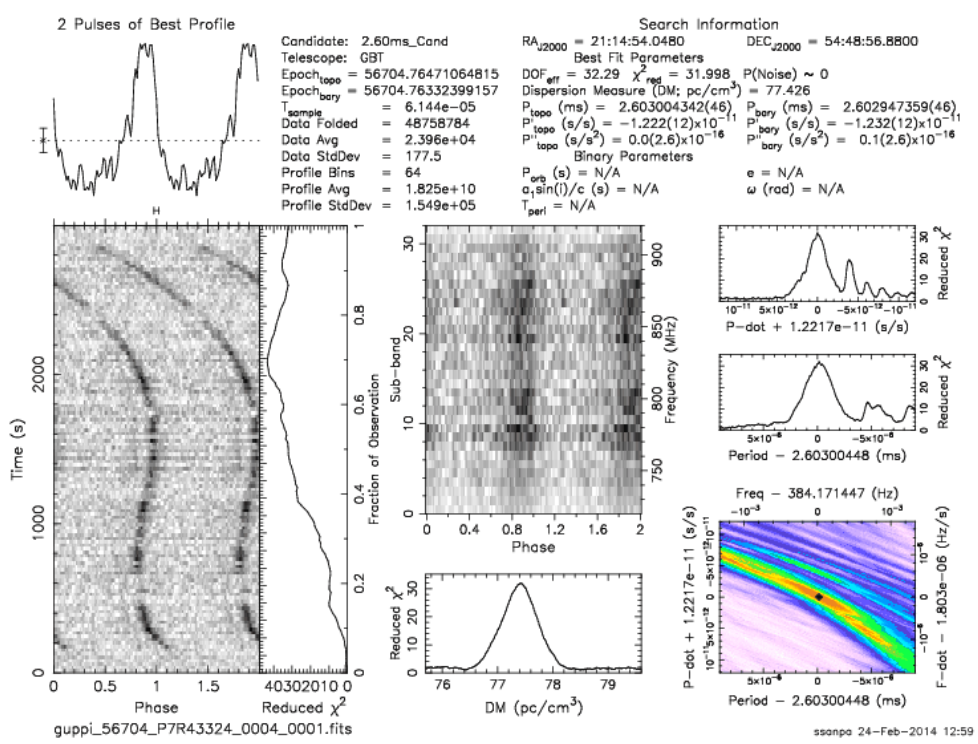

(a)

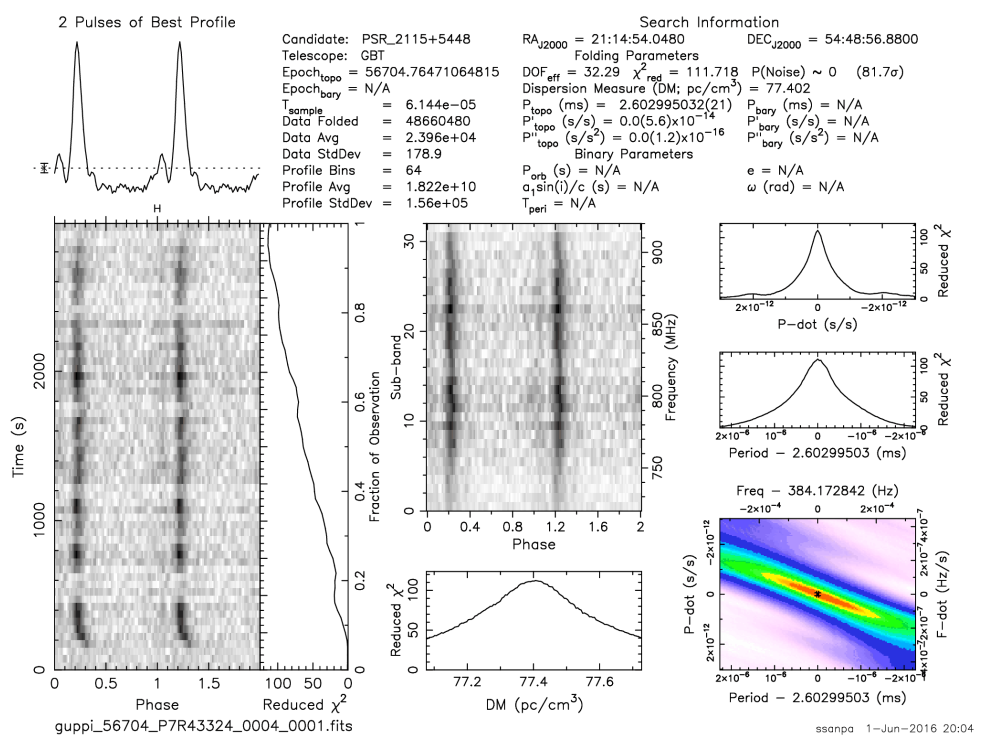

(b)

Fig. 3.9.- : The discovery plot of PSR J2115+5448. PSR J2115+5448 was accelerated by the orbital motion, which resulted in curvature of the pulsar-like signal as shown in Fig.3.9a. The curvature is corrected with the parameters from pulsar timing in Fig3.9b. The pulsar exhibits scintillations, and possibly also short iregular eclipses throughout the observation. 

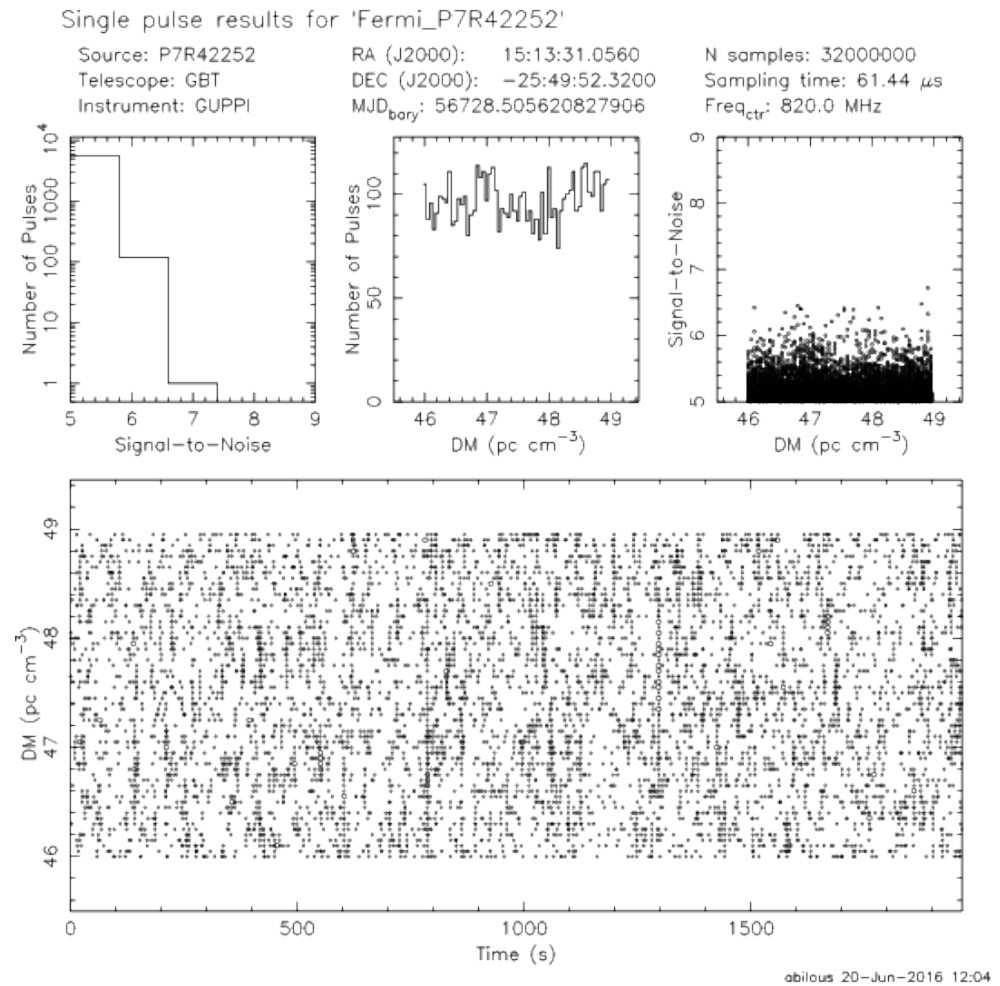

(a)

Single pulse results for 'Fermi_P7R42434'

Source: P7R42434 RA (J2000): 16:22:57.7200

Telescope: GBT DEC (J2000): $\quad-03: 12: 13.6800$ MJD bory: 56733.599196960895

N samples: 42000000

Instrument: GUPPI

Sompling time: $61.44 \mu \mathrm{s}$
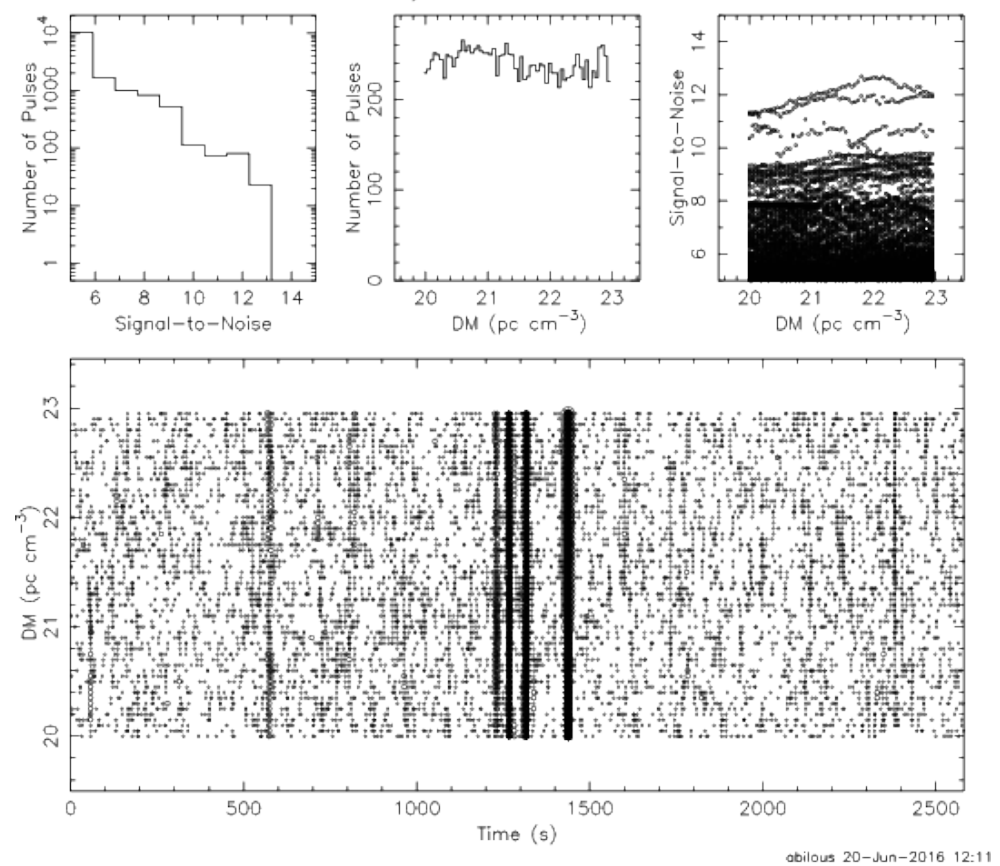

(b)

Fig. 3.10.- : Single-pulse diagnostic plots for PSR J1513-2250 (a) and PSR J1622-0315 (b). No obvious single-pulse candidates were detected at the pulsars' respective DMs of $46.86 \mathrm{pc} \mathrm{cm}^{-3}$ and $21.4 \mathrm{pc} \mathrm{cm}^{-3}$. 

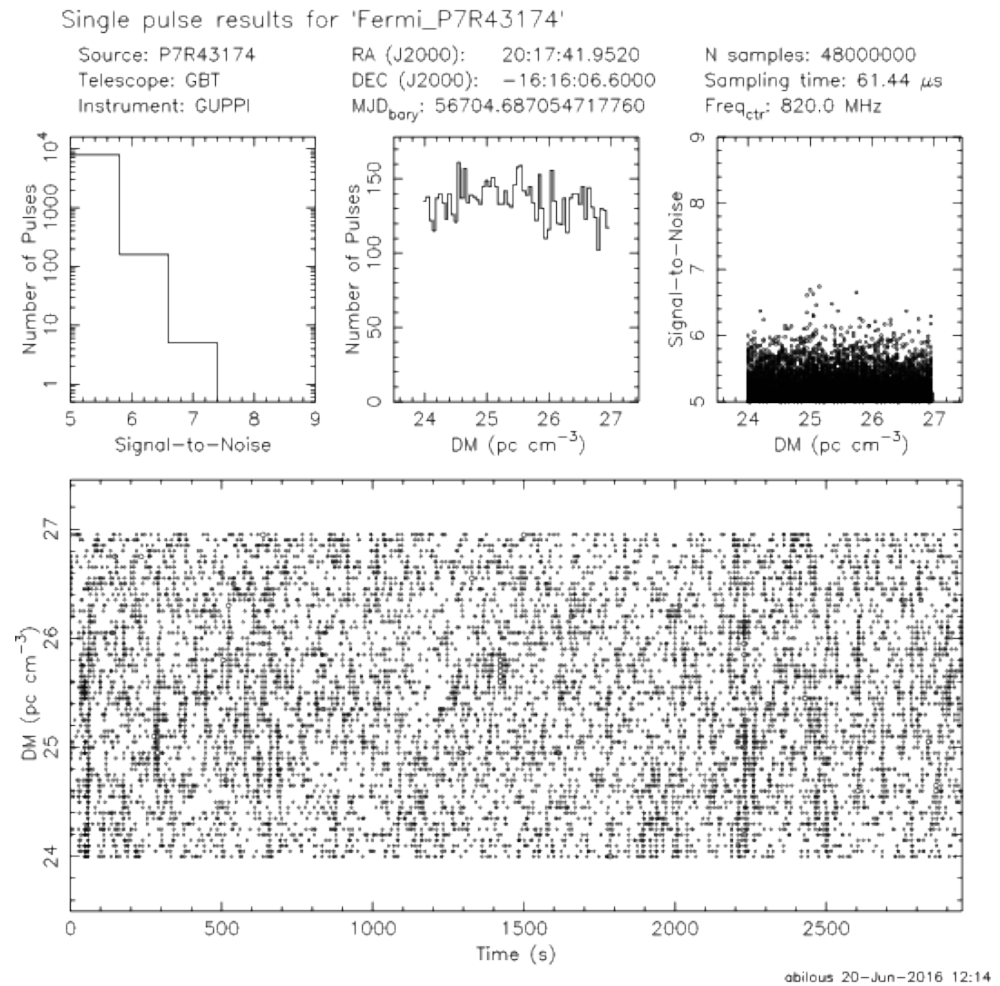

(a)

Single pulse results for 'Fermi_P7R43324'
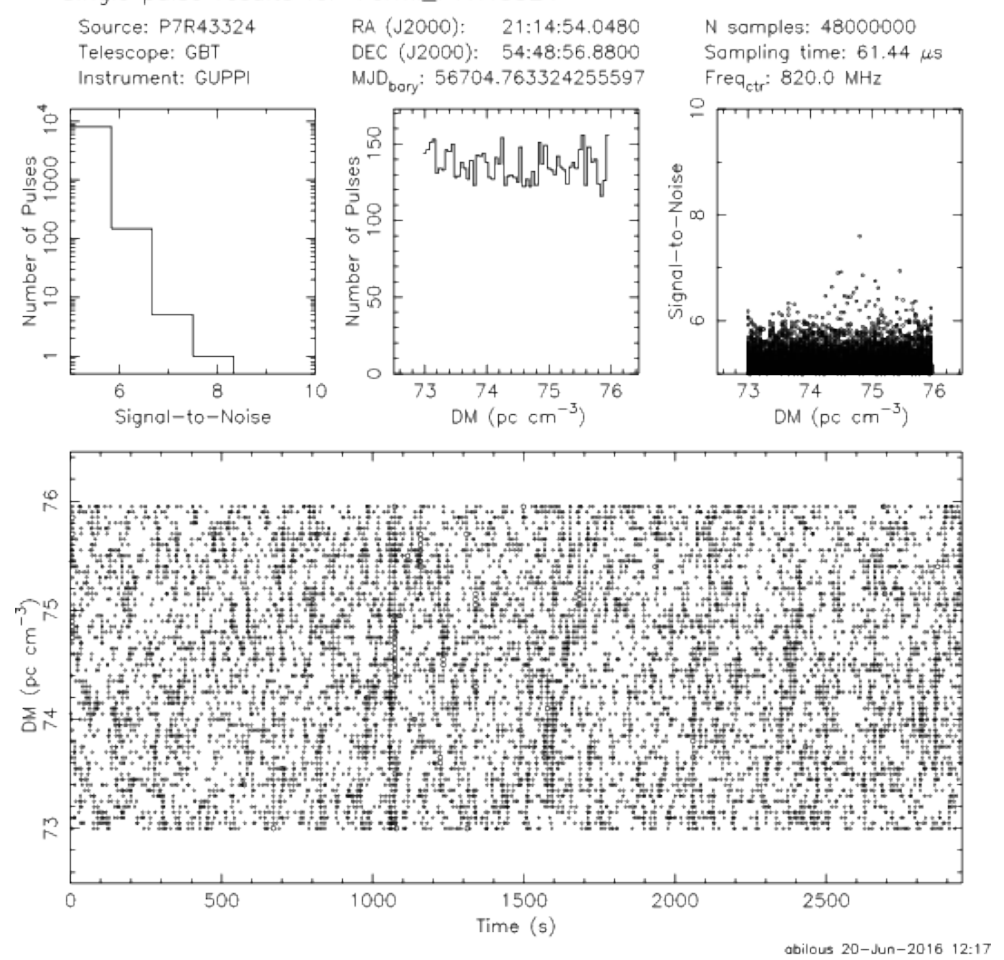

(b)

Fig. 3.11.- : Single-pulse diagnostic plots for PSR J2017-1614 (a) and PSR J2115+5448 (b). No obvious single-pulse candidates were detected at the pulsars' respective DMs of $25.44 \mathrm{pc} \mathrm{cm}^{-3}$ and $77.4 \mathrm{pc} \mathrm{cm}^{-3}$. 


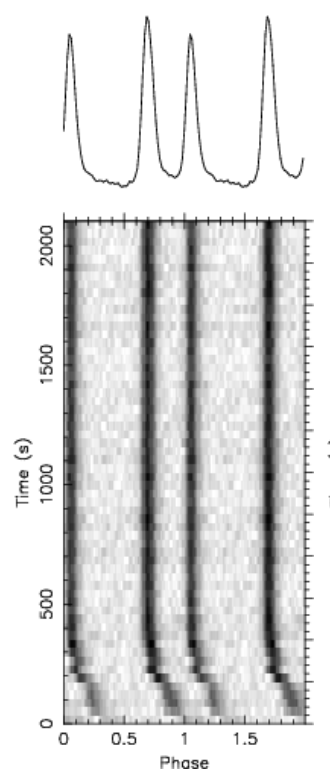

(a)
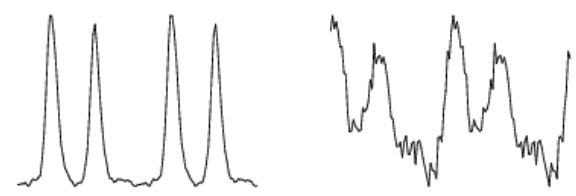

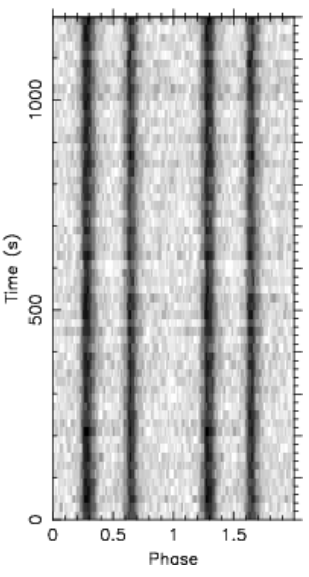

(b)

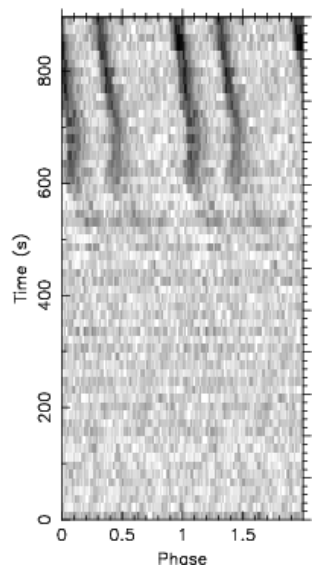

(c)

Fig. 3.12.- : Example of folded pulse profiles PSR J1513-2550 at $820 \mathrm{MHz}$. Panel $3.12 \mathrm{a}$ and $3.12 \mathrm{c}$ show evidence of an eclipse egress at the beginning and two-thirds of the way through the observation, respectively. The pulsar signal is very strong and exhibits no flux density variations at other times. 


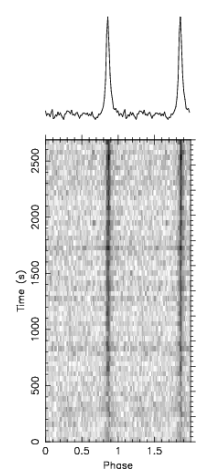

(a)

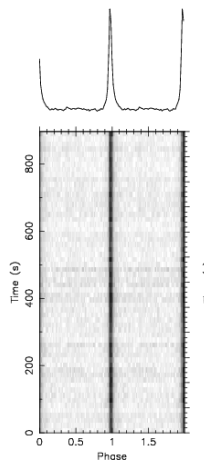

(g)

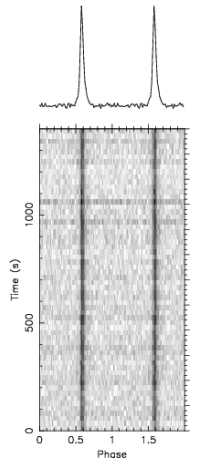

(b)

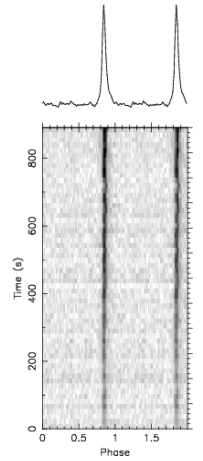

(h)

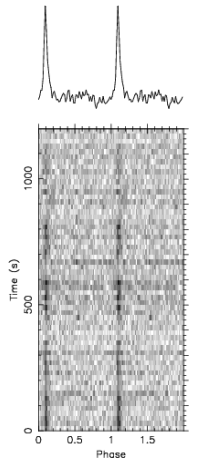

(c)

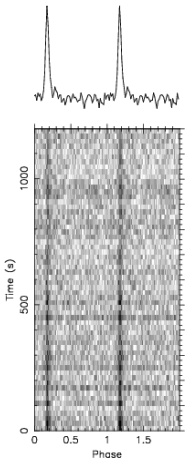

(d)

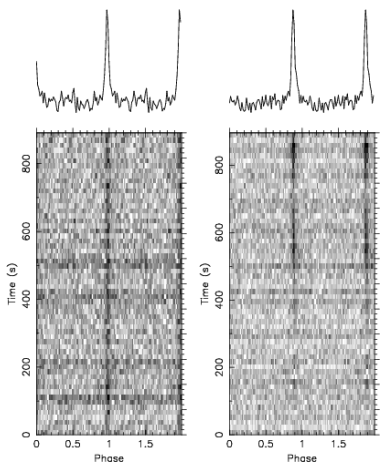

(e)

(f)

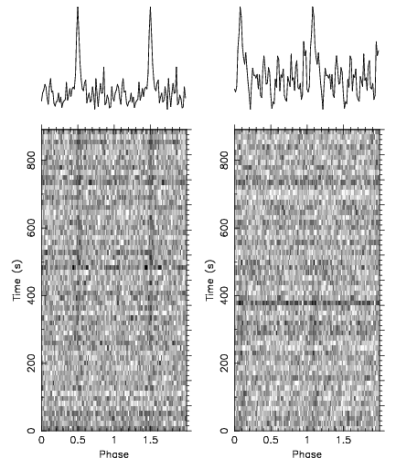

(i)

(j)

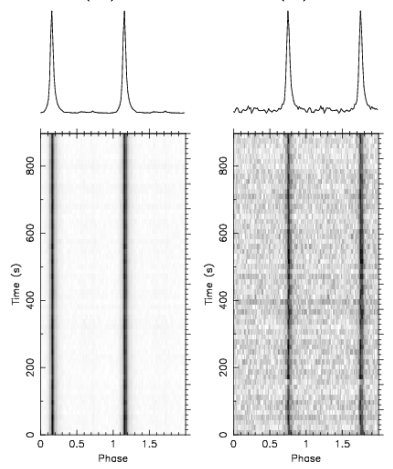

(k)

(l)

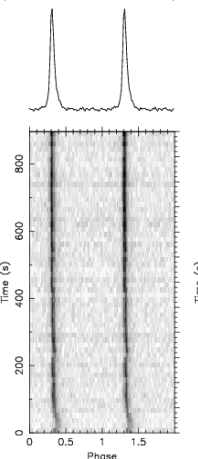

$(\mathrm{m})$
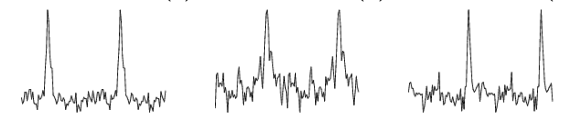

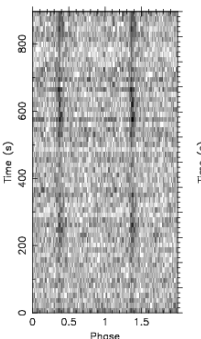

(n)

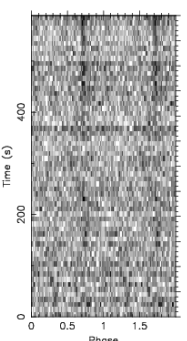

(o)

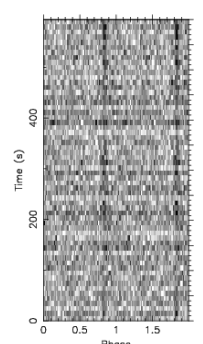

(p)

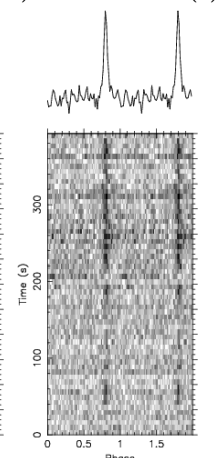

(q)

Fig. 3.13. - : Example of folded pulse profiles of PSR J1622-0315 at $820 \mathrm{MHz}$. Diffractive scintillation is demonstrated by the variability of flux density among same length observations. For instance, the observed intensities of the 15-minute observations from panel 3.13e through 3.13n vary by a factor of $\sim 120(\sim 0.1-12 \mathrm{mJy})$. Additionally, the irregular eclipses (the flux variability within the observation) are exhibited on panel 3.13a, 3.13c, 3.13d, 3.13f, 3.13h, 3.13i, 3.13j and from $3.13 \mathrm{~m}$ to $3.13 q$. 


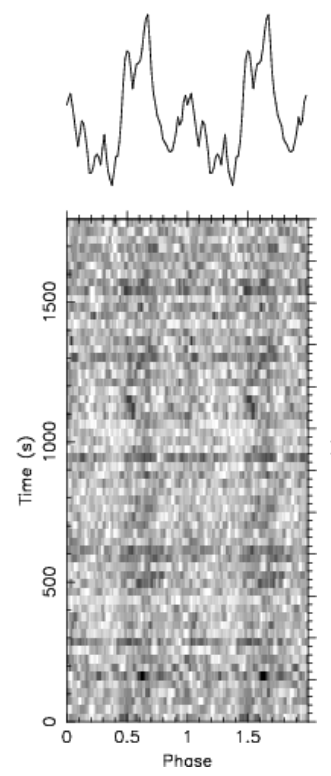

(a) $350 \mathrm{MHz}$
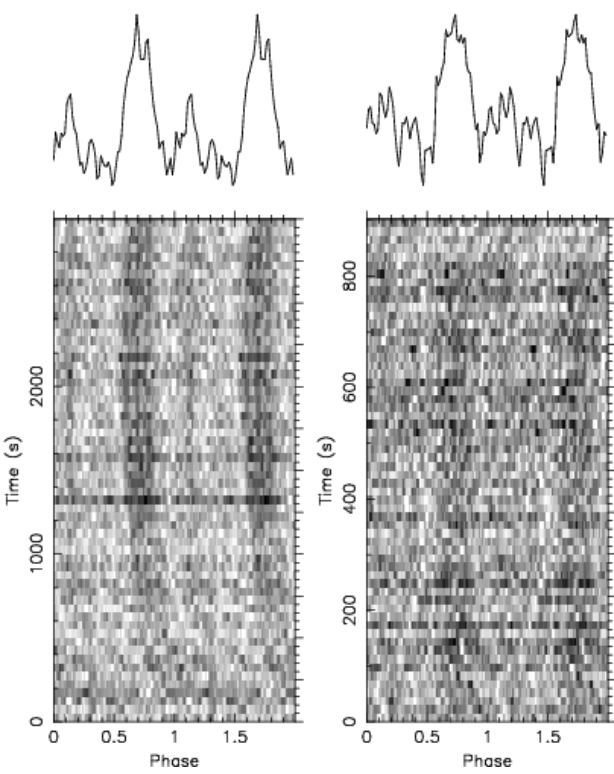

(b) $820 \mathrm{MHz}$

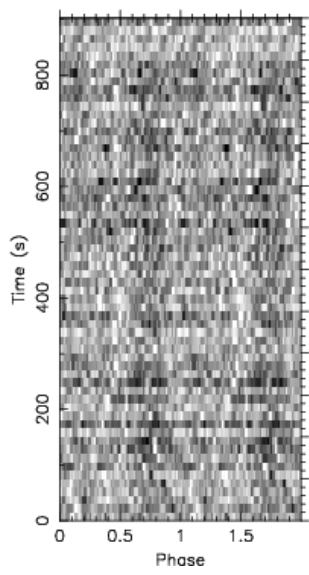

(c) $820 \mathrm{MHz}$

Fig. 3.14.-: Example folded pulse profiles of PSR J2017-1614 at $350 \mathrm{MHz}$ on panel $3.14 \mathrm{a}$ and at $820 \mathrm{MHz}$ on panel $3.14 \mathrm{~b}$ and $3.14 \mathrm{c}$. There is no obvious variation in flux density in different observation with the same length. The panel 3.14b shows the egress from the eclipse about one-third of the way through the observation. 

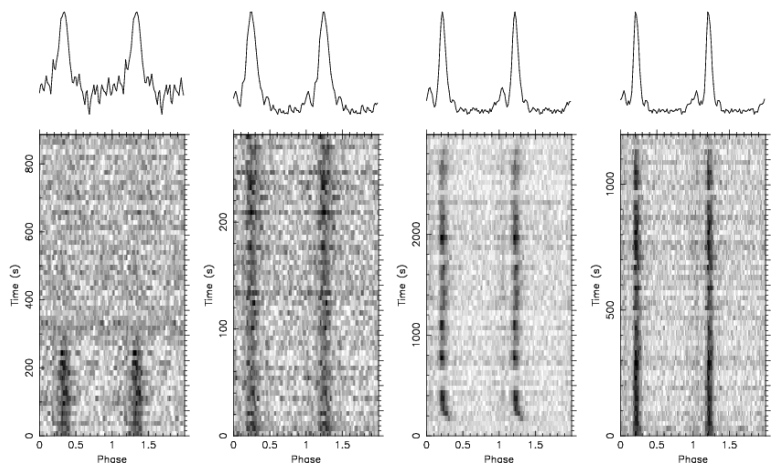

(a) $350 \mathrm{MHz}$

(b) $350 \mathrm{MHz}$

(c) $820 \mathrm{MHz}$

(d) $820 \mathrm{MHz}$
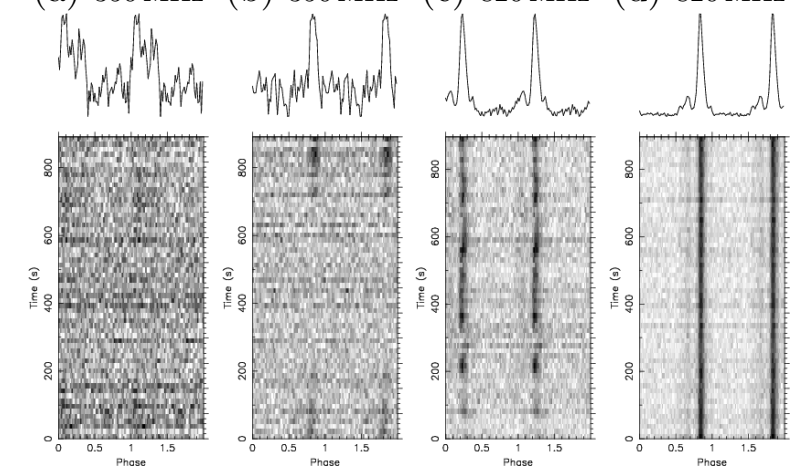

(e) $820 \mathrm{MHz}$

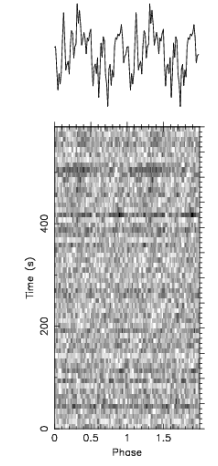

(f) $820 \mathrm{MHz}$

(g) $820 \mathrm{MHz}$

(h) $820 \mathrm{MHz}$
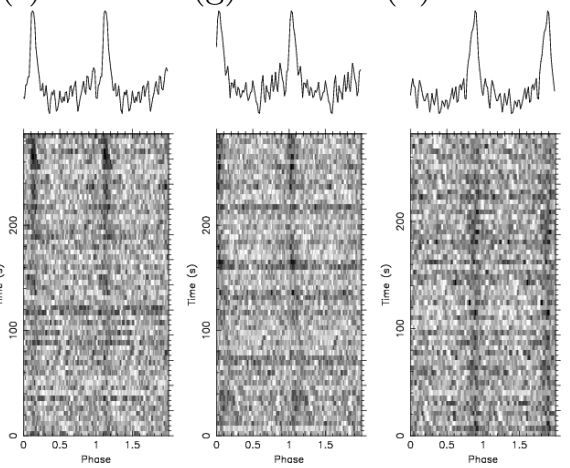

(i) $820 \mathrm{MHz}$

(j) $820 \mathrm{MHz}$

(k) $820 \mathrm{MHz}$

(l) $820 \mathrm{MHz}$

Fig. 3.15.- : Example folded pulse profiles of PSR J2115+5548 at $350 \mathrm{MHz}$ on panel $3.15 \mathrm{a}$ and $3.15 \mathrm{~b}$, and at $820 \mathrm{MHz}$ from panel 3.15c through $3.15 \mathrm{l}$. The panel $3.15 \mathrm{a}$ displays eclipsing ingress at $350 \mathrm{MHz}$ about one third into the observation. There is evidence of eclipsing egress at the beginning of the $820 \mathrm{MHz}$ observation on panel 3.15c. The variations of intensity indicate the scintillation of the pulsar emission. 


\section{Chapter 4}

\section{Conclusions and Future Work}

In this thesis, I present the discovery and analysis of 12 new MSPs with He-WD companions and four new "Spider" MSPs in compact orbits (orbital periods < 1 day). These MSPs were discovered by searching for pulsar-like signals within the error boxes of FermiLAT unassociated sources with the GBT. For all of the MSPs timing solutions were established, and $\gamma$-ray pulsations were detected for twelve of them.

For the timing analyses of the 12 He-WD MSP systems, we were able to determine better measurements of $\dot{P}$ for several of the pulsars using a new MCMC single-photon timing technique over the full Fermi mission. One of the brightest pulsars, with the lowest timing rms residuals, has already been added to the NANOGrav timing array. We performed single-pulse searches on all 198 Fermi LAT sources and also searched 20 sources near the Galactic plane for young pulsars. However, the results were negative in both cases. We confirmed that there is almost no correlation between $\gamma$-ray and radio flux density, which suggests the possibility of finding new bright radio pulsars by searching faint $\gamma$-ray sources. Additionally, we have examined the $P_{b}-M_{c}$ relation, including the twelve new pulsars with He-WD companions, and 
found that the distribution of binary inclination angles $i$ may be skewed towards lower inclinations (as opposed to $\cos i$ being drawn from a uniform distribution, as would be the case if orbits are randomly distributed).

The four new "spider" pulsars (those thought to be missing links in pulsar evolution), exhibit regular eclipses and/or random short-duration eclipses. Such behaviour is the signature of Black Widow and Redback systems. Two of the four pulsars show extremely strong diffractive scintillation at our $820 \mathrm{MHz}$ observing frequency. For two of the MSPs we found optical counterparts. One of them shows modulation in its light curve with a period equal to half of $P_{b}$, which indicates ellipsoidal distortion of the swollen companion.

We plan to perform optical observations of the four spiders in September 2016 to (1) obtain more data on those that are marginally detected, and (2) to acquire multi-color light curves for further more detailed studies. These studies will be led by Jules Halpern. Additionally, we were granted 80 hours of GBT time in the Fall 2016 semester to search for even more pulsars towards Fermi unassociated sources. We expect to find new MSPs and possibly other exotic pulsar systems as part of this project.

With a number of new, state-of-the-art radio telescopes achieving first light in the near future (e.g. FAST and SKA), we will extend pulsar timing baselines and reduce the rms timing residuals of known pulsars, as well as search for new sources. Now that the Gravitational Wave $(\mathrm{GW})$ window on the universe has opened (e.g. Abbott et al. 2016b,a $)^{1}$, discovering more MSPs is crucial to improve the sensitivity of the Pulsar Timing Arrays that will be used to directly detect other longer-wavelength GWs. It is not an exaggeration to say that the Fermi LAT sources are gifts that keep

\footnotetext{
${ }^{1}$ Note that the LIGO project is sensitive to GW frequencies on the order of hundreds of $\mathrm{Hz}$ (such as those produced by NS-NS or BH-BH mergers), while PTAs are sensitive to GW frequencies in the nanohertz regime (such as those produced by SMBH binaries).
} 
on giving, and that the best of the pulsar era is yet to come. 
Chapter 5

Appendix: Tables 


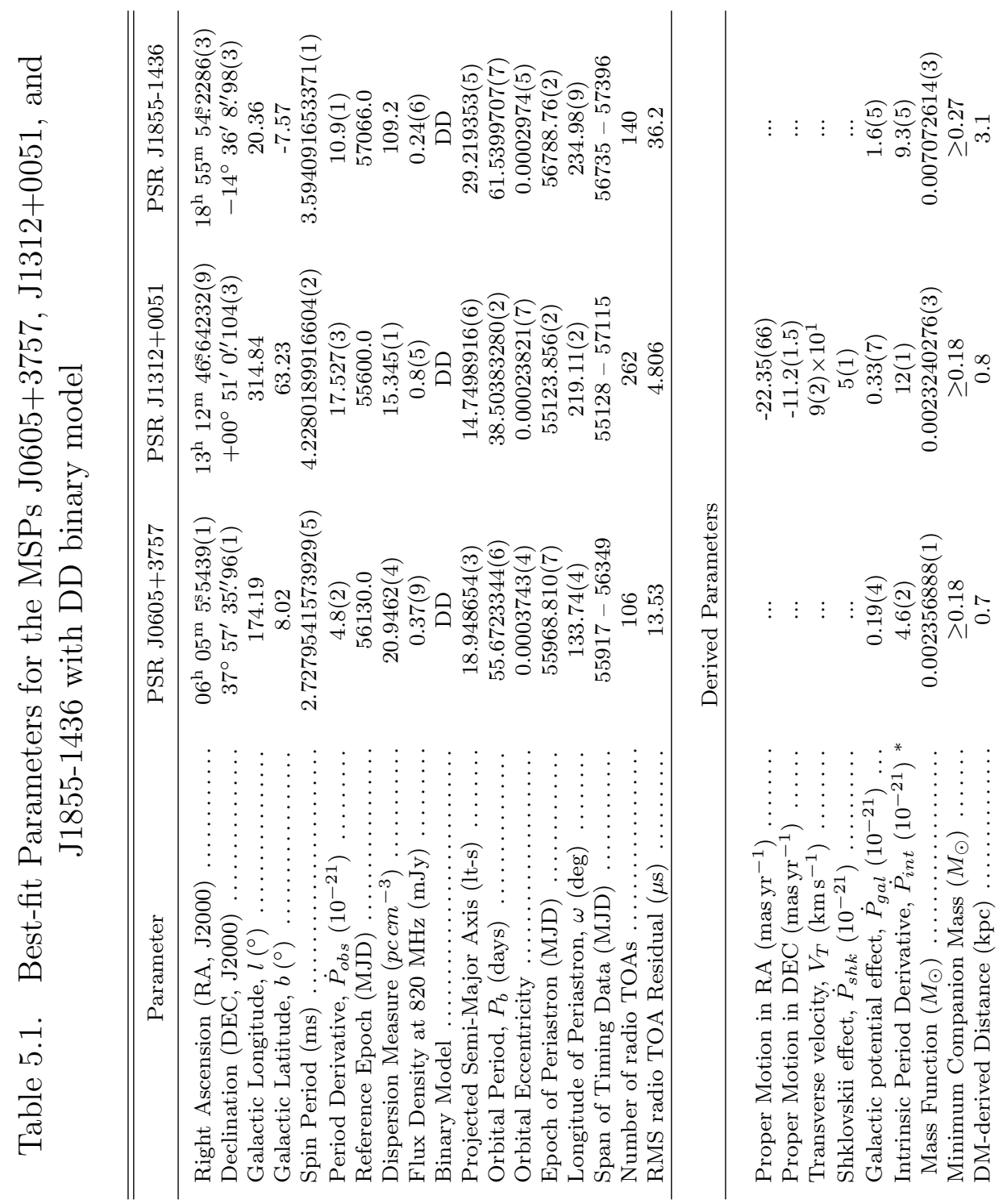




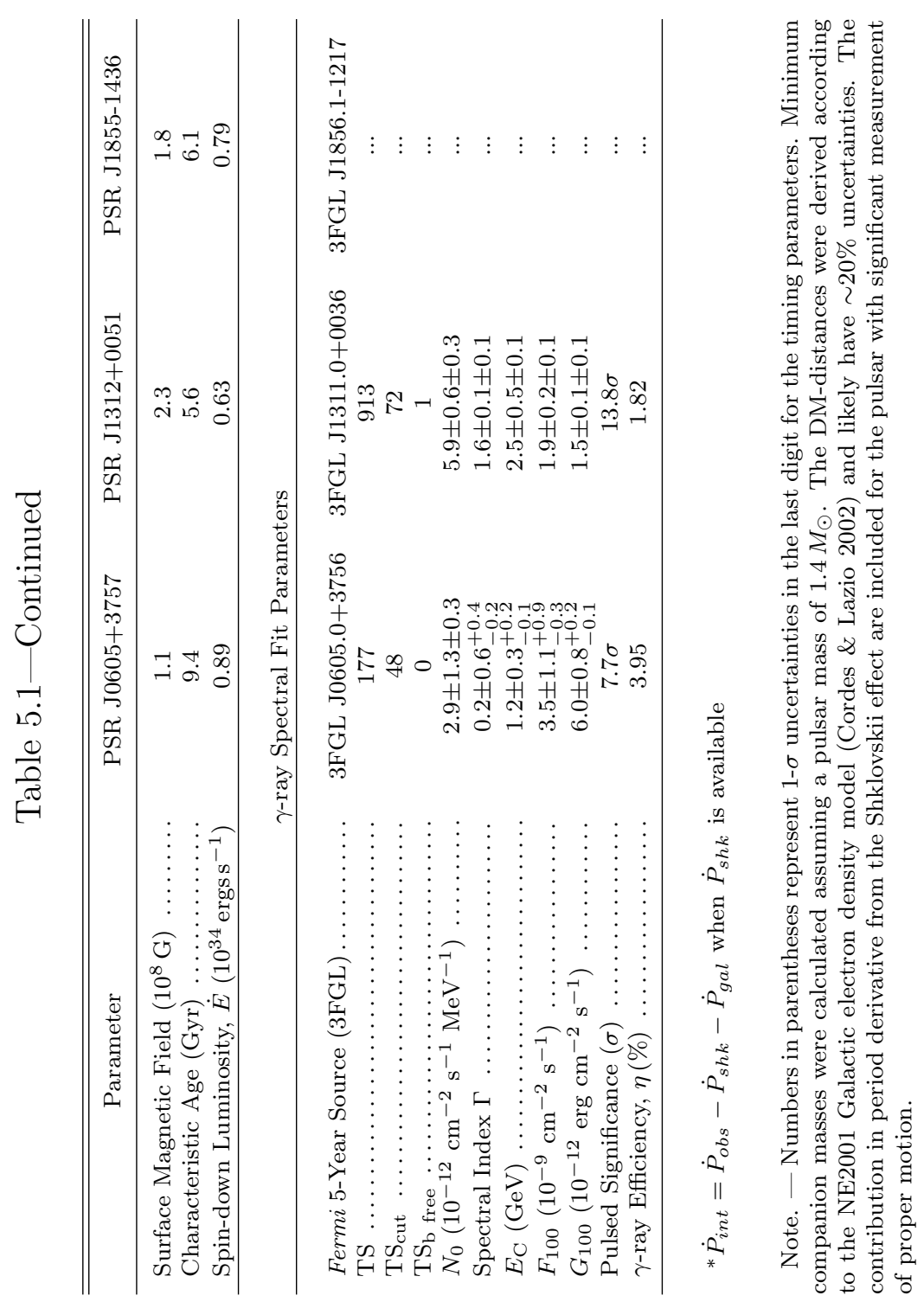


Table 5.2. Best-fit Parameters for the Isolated MSP J0533+6759

\begin{tabular}{|c|c|}
\hline Parameter & PSR J0533+6759 \\
\hline Right Ascension (RA, J2000) & $05^{\mathrm{h}} 33^{\mathrm{m}} 55^{\mathrm{s}} 1401(3)$ \\
\hline Declination (DEC, J2000) & $67^{\circ} 59^{\prime} 17^{\prime \prime} 489(3)$ \\
\hline Galactic Longitude, $l\left(^{\circ}\right)$ & 144.78 \\
\hline Galactic Latitude, $b\left(^{\circ}\right)$. & 18.18 \\
\hline Spin Period $(\mathrm{ms}) \ldots \ldots$ & $4.3881599698083(9)$ \\
\hline Period Derivative, $\dot{P}_{o b s}\left(10^{-21}\right)$ & $12.61(9)$ \\
\hline Reference Epoch (MJD) ... & 55877.0 \\
\hline Dispersion Measure $\left(p c c m^{-3}\right)$ & $57.369(2)$ \\
\hline Flux Density at $820 \mathrm{MHz}(\mathrm{mJy})$. & $0.26(3)$ \\
\hline Span of Timing Data (MJD) .... & $55547-56207$ \\
\hline Number of radio TOAs ..... & 86 \\
\hline RMS radio TOA Residual $(\mu \mathrm{s}) \ldots \ldots \ldots$ & 24.05 \\
\hline \multicolumn{2}{|l|}{ Derived Parameters } \\
\hline Proper Motion in RA $\left(\operatorname{mas~yr}^{-1}\right)$ & $\ldots$ \\
\hline Proper Motion in DEC (mas yr $\left.{ }^{-1}\right)$ & $\ldots$ \\
\hline Transverse velocity, $V_{T}\left(\mathrm{~km} \mathrm{~s}^{-1}\right) \ldots$ & $\ldots$ \\
\hline Shklovskii effect, $\dot{P}_{s h k}\left(10^{-21}\right) \ldots$ & $\ldots$ \\
\hline Galactic potential effect, $\dot{P}_{g a l}\left(10^{-21}\right)$ & $1.3(4)$ \\
\hline Intrinsic Period Derivative, $\dot{P}_{\text {int }}\left(10^{-21}\right)^{*}$ & $11.3(4)$ \\
\hline DM-derived Distance $(\mathrm{kpc}) \ldots \ldots \ldots \ldots$ & 2.4 \\
\hline Surface Magnetic Field $\left(10^{8} \mathrm{G}\right) \ldots$ & 2.3 \\
\hline Characteristic Age (Gyr) ...... & 6.2 \\
\hline Spin-down Luminosity, $\dot{E}\left(10^{34} \operatorname{ergs~s}^{-1}\right)$ & 0.53 \\
\hline
\end{tabular}

$\gamma$-ray Spectral Fit Parameters

\begin{tabular}{|c|c|}
\hline Fermi 5-Year Source (3FGL). & 3FGL J0534.0+6759 \\
\hline TS & 479 \\
\hline $\mathrm{TS}_{\text {cut }}$ & 31 \\
\hline $\mathrm{TS}_{\mathrm{b} \text { free }}$ & 1 \\
\hline$N_{0}\left(10^{-12} \mathrm{~cm}^{-2} \mathrm{~s}^{-1} \mathrm{MeV}^{-1}\right)$. & $1.1 \pm 0.1_{-0.5}^{+0.6}$ \\
\hline Spectral Index $\Gamma \ldots \ldots \ldots$ & $1.6 \pm 0.1 \pm 0.1$ \\
\hline$E_{\mathrm{C}}(\mathrm{GeV})$ & $5.6 \pm 1.5 \pm 0.4$ \\
\hline$F_{100}\left(10^{-9} \mathrm{~cm}^{-2} \mathrm{~s}^{-1}\right)$. & $9.9 \pm 1.7_{-0.6}^{+0.9}$ \\
\hline$G_{100}\left(10^{-12} \mathrm{erg} \mathrm{cm}^{-2} \mathrm{~s}^{-1}\right)$ & $9.9 \pm 0.8_{-0.5}^{+0.6}$ \\
\hline Pulsed Significance $(\sigma) \ldots \ldots$ & $13.5 \sigma$ \\
\hline$\gamma$-ray Efficiency, $\eta(\%)$ & 128.73 \\
\hline
\end{tabular}

${ }^{*} \dot{P}_{i n t}=\dot{P}_{o b s}-\dot{P}_{s h k}-\dot{P}_{g a l}$ when $\dot{P}_{s h k}$ is available

Note. - Numbers in parentheses represent 1- $\sigma$ uncertainties in the last digit for the timing parameters. Minimum companion masses were calculated assuming a pulsar mass of $1.4 M_{\odot}$. The DM-distances were derived according to the NE2001 Galactic electron density model (Cordes \& Lazio 2002) and likely have $\sim 20 \%$ uncertainties. The contribution in period derivative from the Shklovskii effect are included for the pulsar with significant measurement of proper motion. 


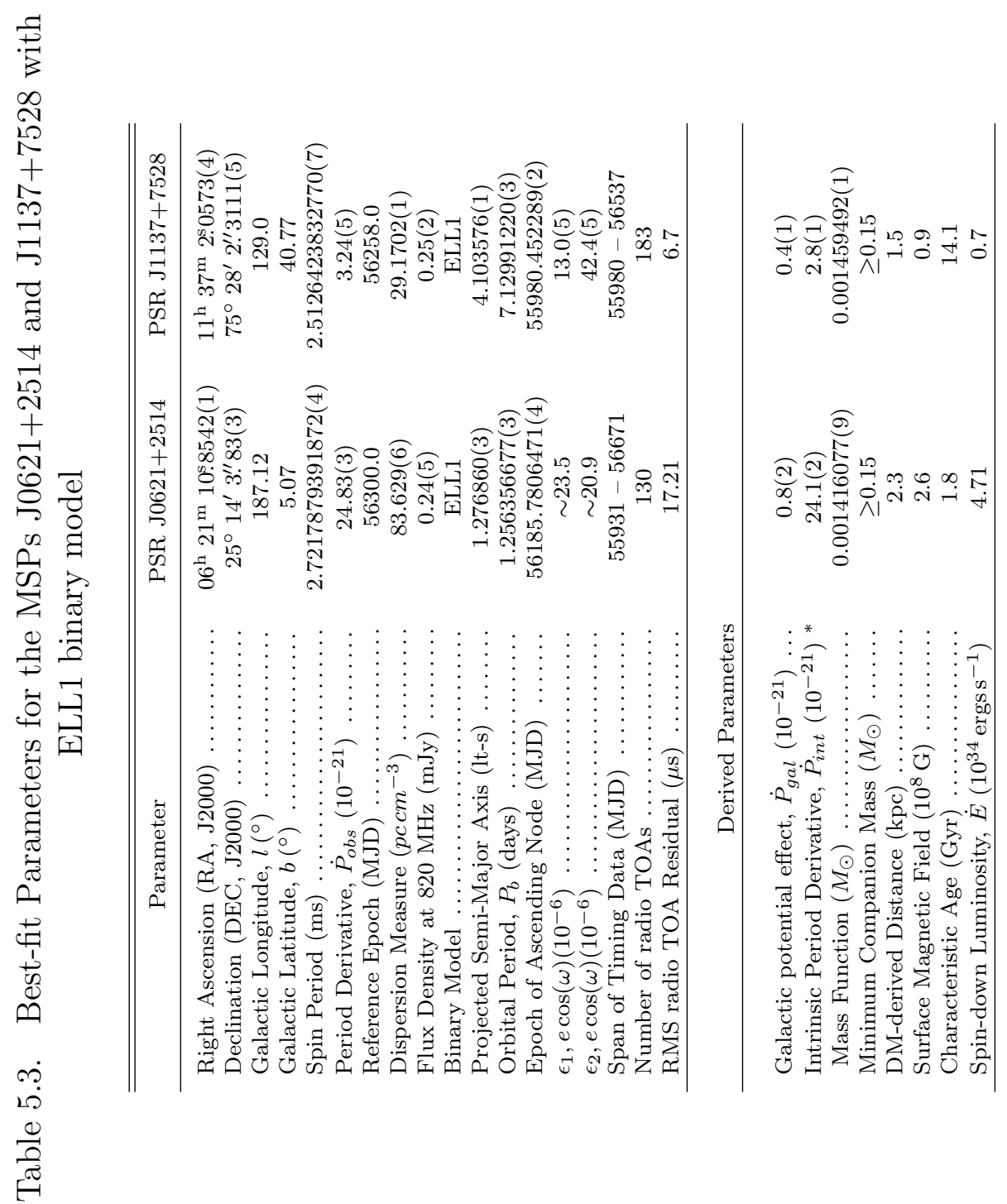




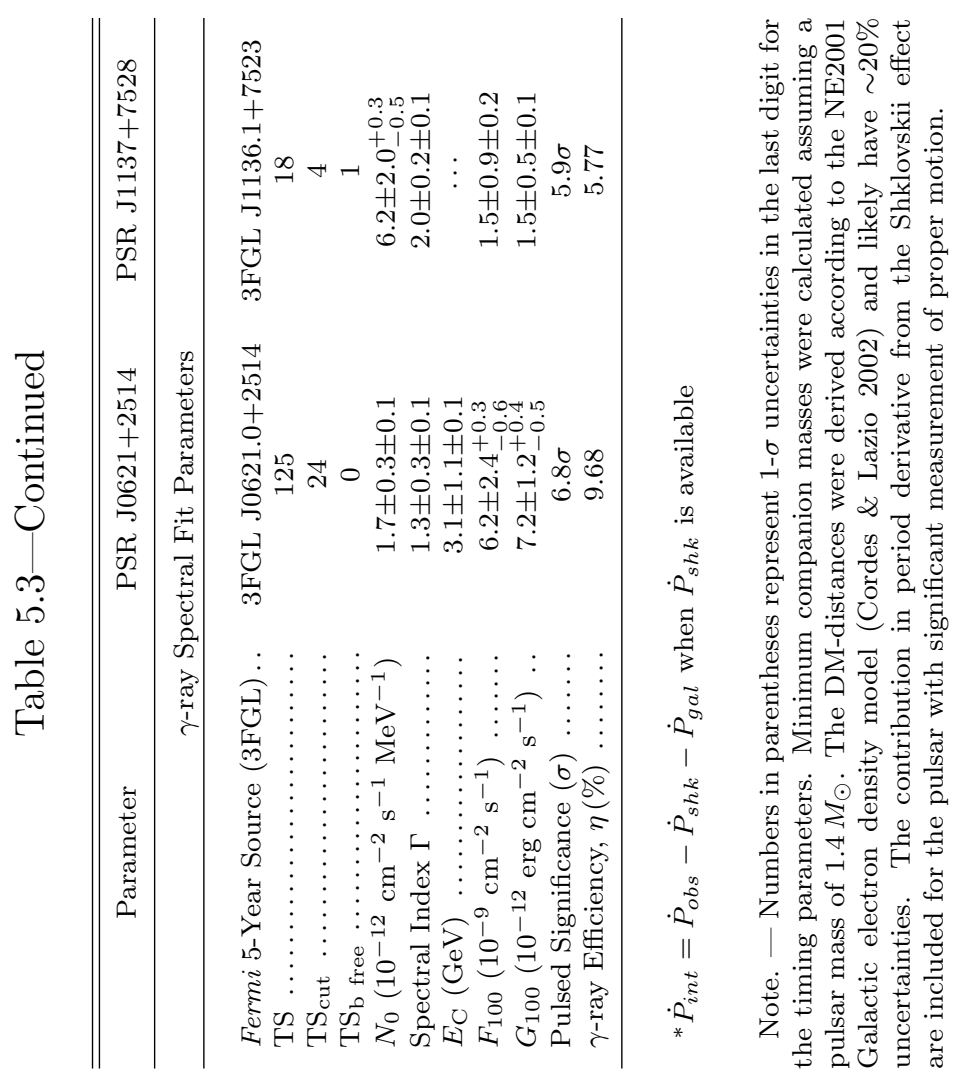




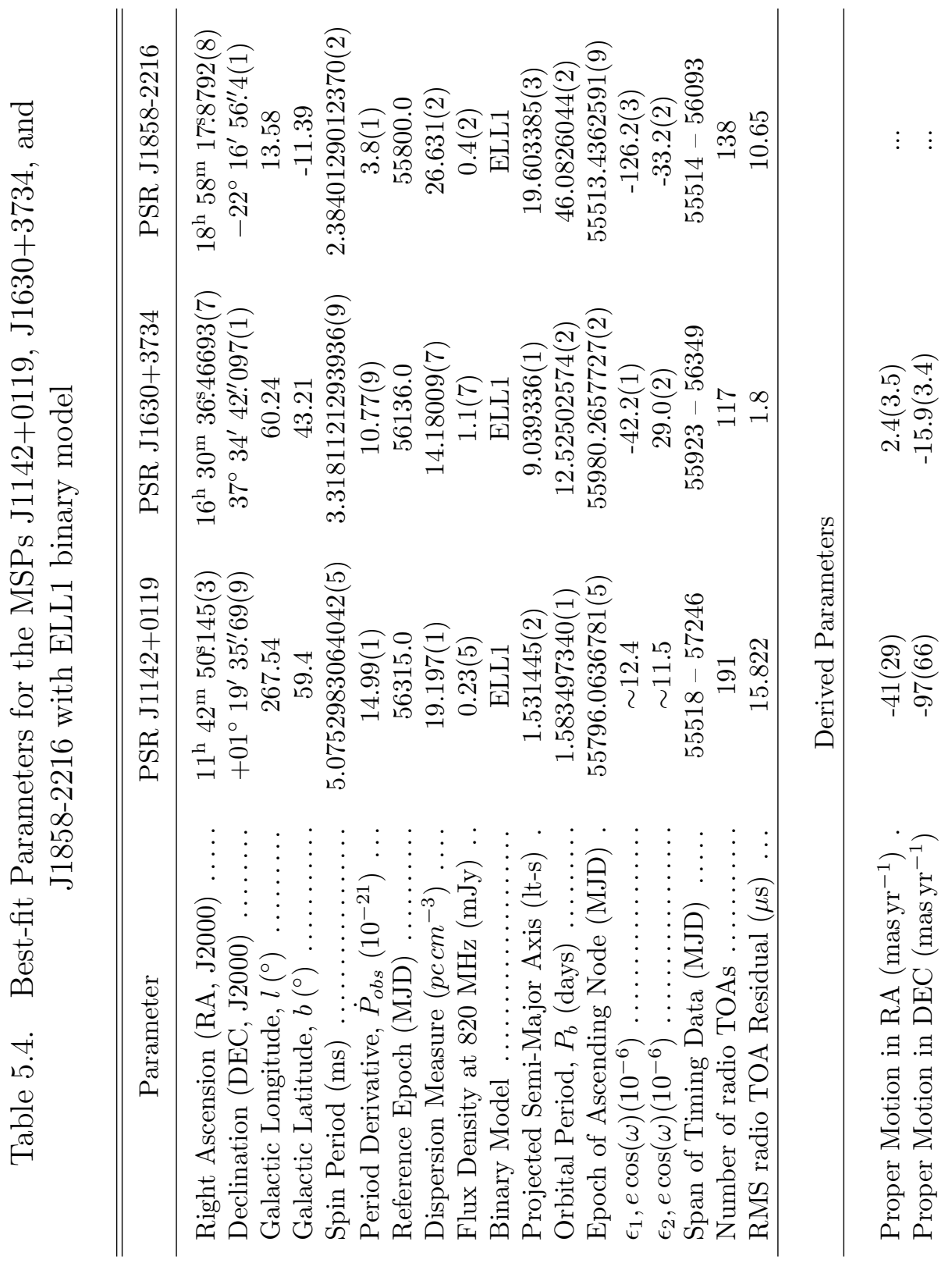




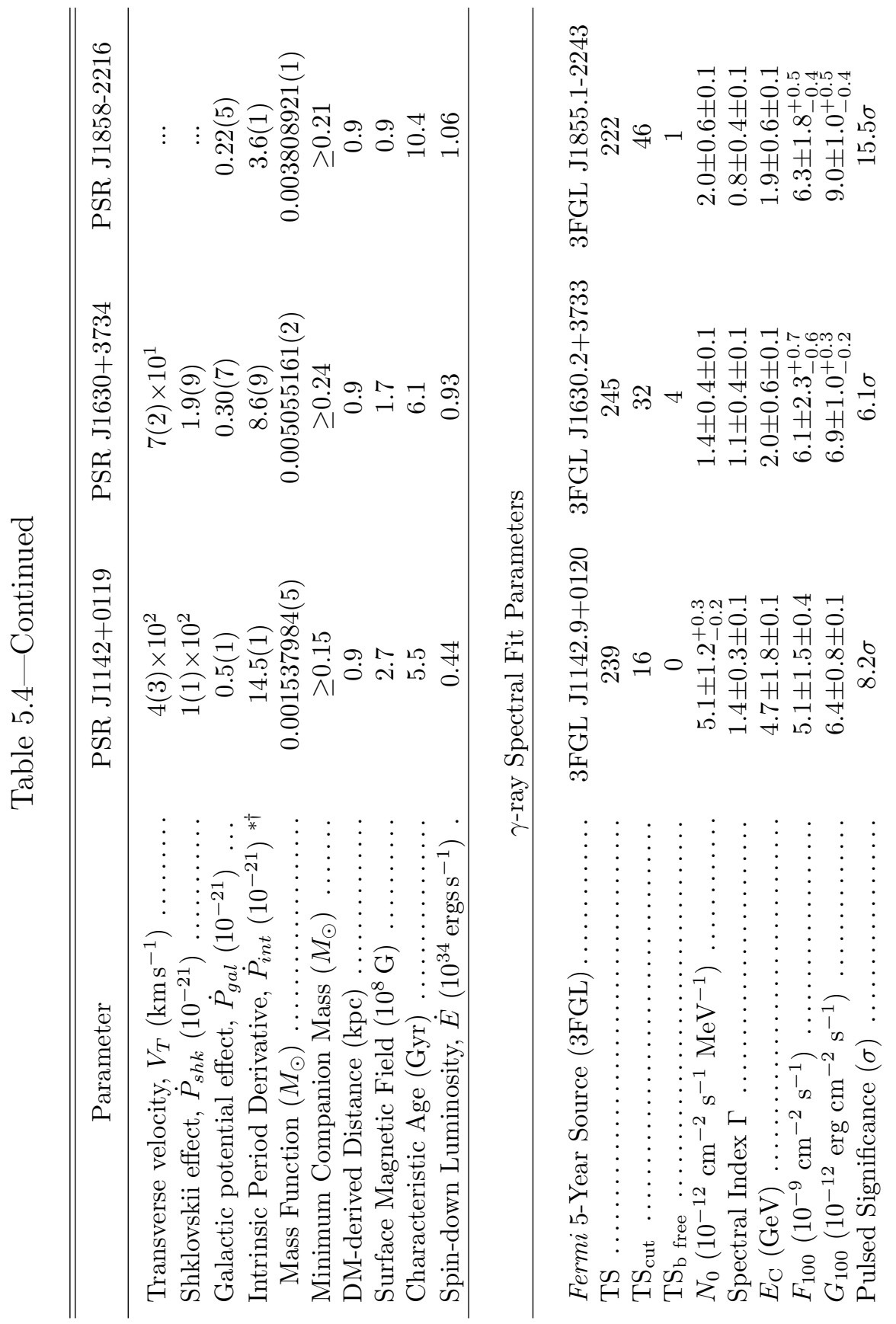




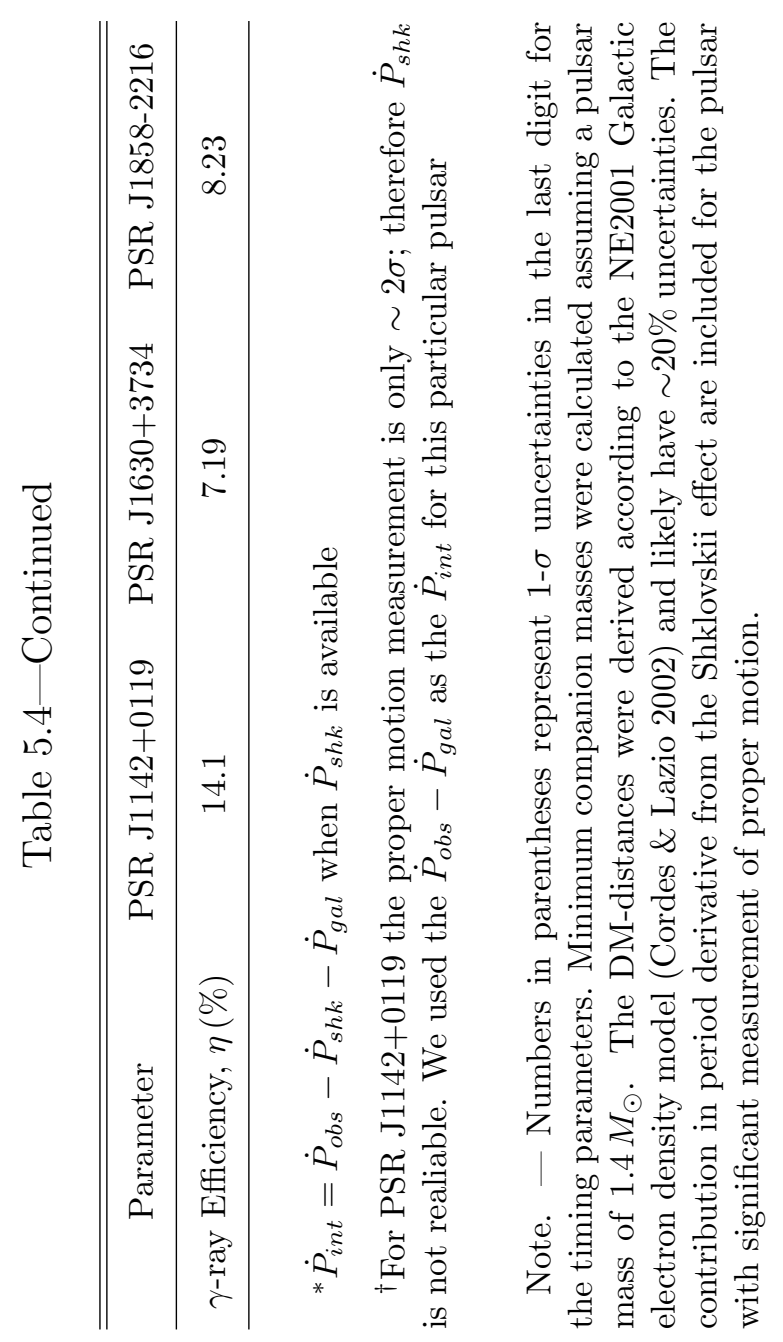




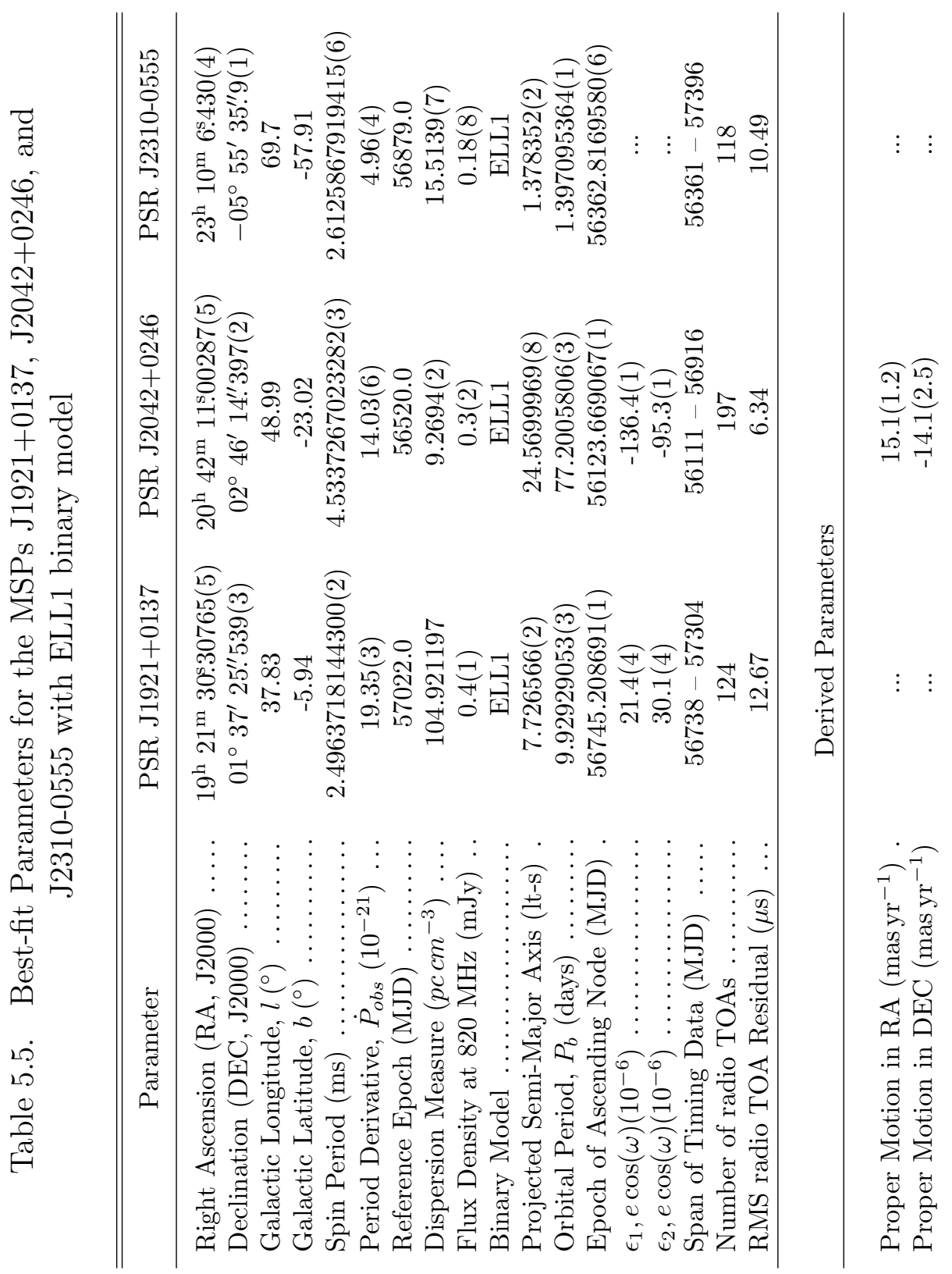




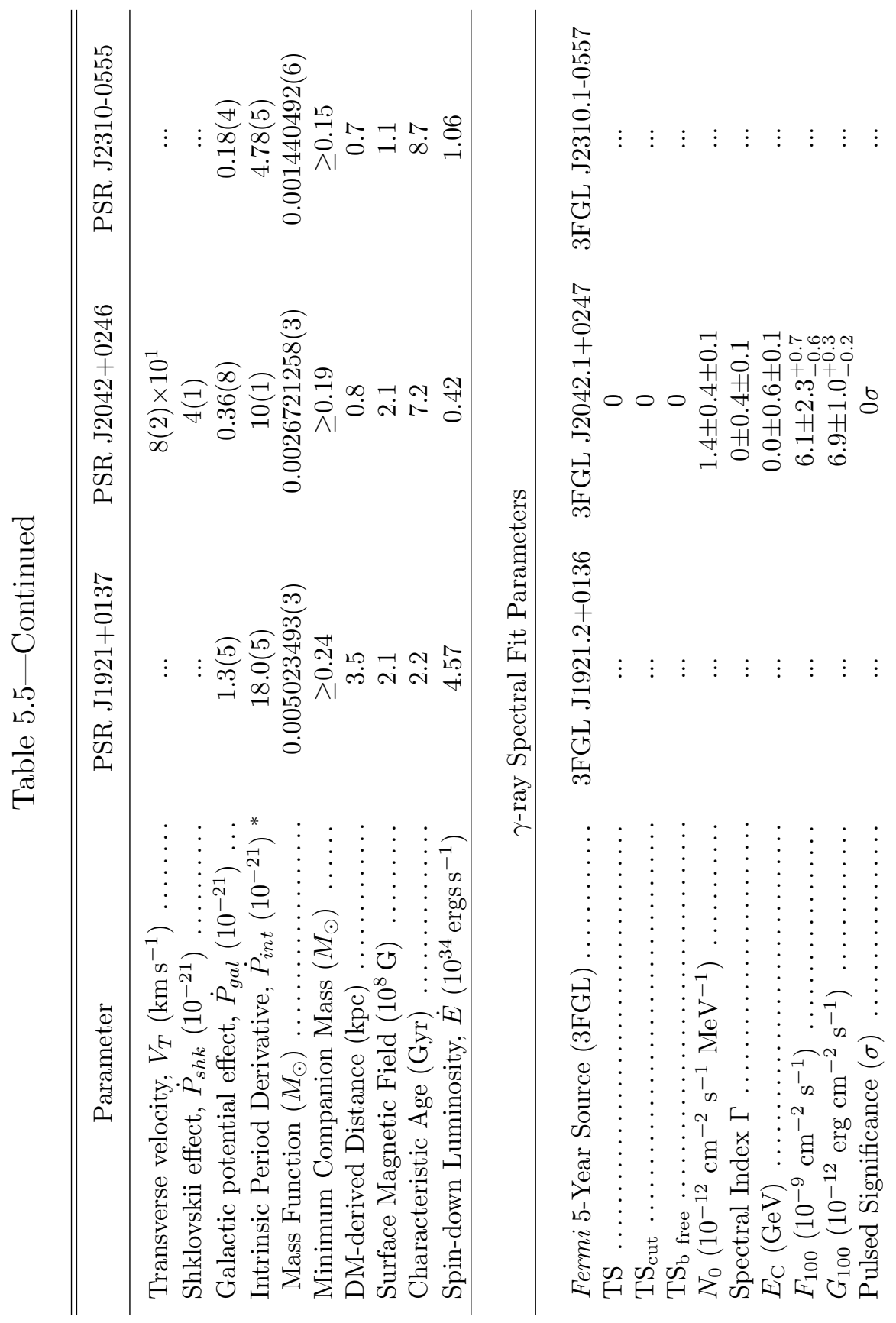




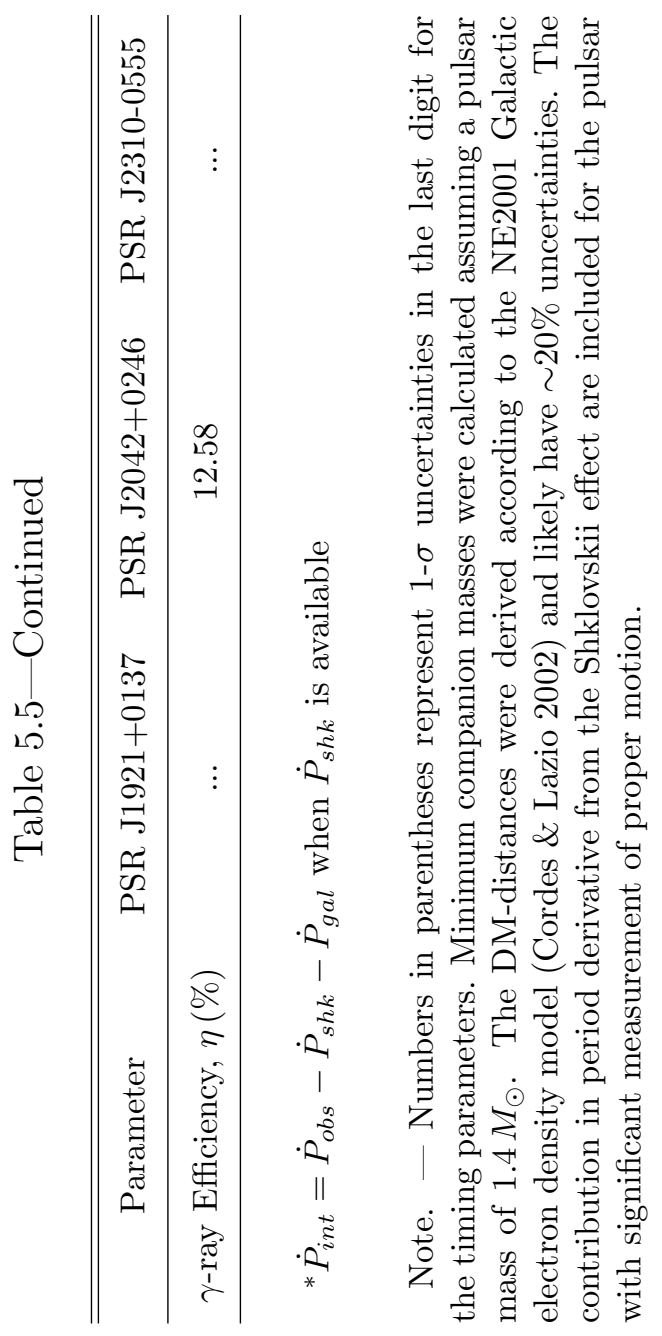


Table 5.6. Radio Searches of Fermi Unassociated Sources with the GBT

\begin{tabular}{|c|c|c|c|c|c|c|c|}
\hline Source & $\begin{array}{c}\text { R.A. } \\
\text { J2000.0 }\end{array}$ & $\begin{array}{c}\text { Decl. } \\
\text { J2000.0 }\end{array}$ & $\begin{array}{c}l \\
\operatorname{deg}\end{array}$ & $\begin{array}{c}b \\
\operatorname{deg}\end{array}$ & $\begin{array}{l}\text { Integra- } \\
\text { tion Time } \\
\text { minutes }\end{array}$ & $\begin{array}{c}\text { Observing } \\
\text { Freq } \\
\mathrm{MHz}\end{array}$ & 3FGL name \\
\hline P7R40016 & $00^{\mathrm{h}} 06^{\mathrm{m}} 17^{\mathrm{s}}$ & $+01^{\circ} 35^{\prime} 08^{\prime \prime}$ & 100.4 & -59.3 & 35 & 350 & 3FGL J0006.2+0135 \\
\hline P72Y0024 & $00^{\mathrm{h}} 08^{\mathrm{m}} 07^{\mathrm{s}}$ & $+68^{\circ} 33^{\prime} 49^{\prime \prime}$ & 119.0 & 6.0 & 55 & 820 & 3FGL J0008.5+6853 \\
\hline P72Y0024 & $00^{\mathrm{h}} 08^{\mathrm{m}} 07^{\mathrm{s}}$ & $+68^{\circ} 33^{\prime} 50^{\prime \prime}$ & 119.0 & 6.0 & 50 & 820 & 3FGL J0008.5+6853 \\
\hline P7R40023 & $00^{\mathrm{h}} 08^{\mathrm{m}} 21^{\mathrm{s}}$ & $+14^{\circ} 56^{\prime} 00^{\prime \prime}$ & 107.7 & -46.7 & 35 & 350 & 3FGL J0008.3+1456 \\
\hline P7R40031 * & $00^{\mathrm{h}} 10^{\mathrm{m}} 30^{\mathrm{s}}$ & $-14^{\circ} 25^{\prime} 10^{\prime \prime}$ & 84.0 & -74.1 & 33 & 350 & 3FGL J0010.5-1425 \\
\hline P7R40076 & $00^{\mathrm{h}} 28^{\mathrm{m}} 38^{\mathrm{s}}$ & $+75^{\circ} 07^{\prime} 06^{\prime \prime}$ & 121.4 & 12.3 & 45 & 820 & 3FGL J0028.6+7507 \\
\hline P7R40076 & $00^{\mathrm{h}} 28^{\mathrm{m}} 38^{\mathrm{s}}$ & $+75^{\circ} 07^{\prime} 06^{\prime \prime}$ & 121.4 & 12.3 & 45 & 820 & 3 FGL J0028.6+7507 \\
\hline 1FGL J0039.2+4331 & $00^{\mathrm{h}} 39^{\mathrm{m}} 01^{\mathrm{s}}$ & $+43^{\circ} 28^{\prime} 08^{\prime \prime}$ & 120.5 & -19.3 & 29 & 820 & None \\
\hline 1FGL J0039.2+4331 & $00^{\mathrm{h}} 39^{\mathrm{m}} 01^{\mathrm{s}}$ & $+43^{\circ} 28^{\prime} 08^{\prime \prime}$ & 120.5 & -19.3 & 5 & 820 & None \\
\hline 2FGL J0039.1+4331 & $00^{\mathrm{h}} 39^{\mathrm{m}} 07^{\mathrm{s}}$ & $+43^{\circ} 31^{\prime} 38^{\prime \prime}$ & 120.6 & -19.3 & 50 & 820 & 3FGL J0039.1+4330 \\
\hline 1FGL J0046.8+5658 & $00^{\mathrm{h}} 47^{\mathrm{m}} 02^{\mathrm{s}}$ & $+56^{\circ} 56^{\prime} 50^{\prime \prime}$ & 122.3 & -5.9 & 5 & 820 & None \\
\hline 1FGL J0054.9-2455 & $00^{\mathrm{h}} 54^{\mathrm{m}} 59^{\mathrm{s}}$ & $-24^{\circ} 52^{\prime} 23^{\prime \prime}$ & 142.6 & -87.6 & 5 & 820 & None \\
\hline 1FGL J0106.7+4853 & $01^{\mathrm{h}} 06^{\mathrm{m}} 35^{\mathrm{s}}$ & $+48^{\circ} 55^{\prime} 29^{\prime \prime}$ & 125.5 & -13.9 & 45 & 820 & None \\
\hline 1FGL J0106.7+4853 & $01^{\mathrm{h}} 06^{\mathrm{m}} 35^{\mathrm{s}}$ & $+48^{\circ} 55^{\prime} 29^{\prime \prime}$ & 125.5 & -13.9 & 5 & 820 & None \\
\hline 1FGL J0106.7+4853 & $01^{\mathrm{h}} 06^{\mathrm{m}} 35^{\mathrm{s}}$ & $+48^{\circ} 55^{\prime} 30^{\prime \prime}$ & 125.5 & -13.9 & 45 & 820 & None \\
\hline P72Y0218 & $01^{\mathrm{h}} 27^{\mathrm{m}} 11^{\mathrm{s}}$ & $-08^{\circ} 15^{\prime} 52^{\prime \prime}$ & 148.7 & -69.3 & 50 & 820 & 3FGL J0127.1-0818 \\
\hline 1FGL J0134.4+2632 & $01^{\mathrm{h}} 34^{\mathrm{m}} 36^{\mathrm{s}}$ & $+26^{\circ} 35^{\prime} 45^{\prime \prime}$ & 134.8 & -35.3 & 45 & 820 & None \\
\hline 1FGL J0134.4+2632 & $01^{\mathrm{h}} 34^{\mathrm{m}} 36^{\mathrm{s}}$ & $+26^{\circ} 35^{\prime} 45^{\prime \prime}$ & 134.8 & -35.3 & 5 & 820 & None \\
\hline P7R40251 & $01^{\mathrm{h}} 48^{\mathrm{m}} 20^{\mathrm{s}}$ & $+52^{\circ} 00^{\prime} 36^{\prime \prime}$ & 131.8 & -9.9 & 45 & 820 & 3 FGL J0148.3+5200 \\
\hline P72Y0261 & $01^{\mathrm{h}} 53^{\mathrm{m}} 07^{\mathrm{s}}$ & $+75^{\circ} 16^{\prime} 01^{\prime \prime}$ & 126.9 & 12.9 & 50 & 820 & 3FGL J0152.8+7517 \\
\hline P72Y0294 & $02^{\mathrm{h}} 03^{\mathrm{m}} 46^{\mathrm{s}}$ & $+30^{\circ} 42^{\prime} 27^{\prime \prime}$ & 140.8 & -29.6 & 40 & 820 & 3FGL J0203.6+3043 \\
\hline 1FGL J0203.5+3044 & $02^{\mathrm{h}} 03^{\mathrm{m}} 57^{\mathrm{s}}$ & $+30^{\circ} 45^{\prime} 17^{\prime \prime}$ & 140.8 & -29.6 & 5 & 820 & None \\
\hline P7R40308 * & $02^{\mathrm{h}} 11^{\mathrm{m}} 16^{\mathrm{s}}$ & $-06^{\circ} 49^{\prime} 42^{\prime \prime}$ & 169.6 & -62.2 & 50 & 350 & 3FGL J0211.2-0649 \\
\hline P7R40312 * & $02^{\mathrm{h}} 12^{\mathrm{m}} 08^{\mathrm{s}}$ & $+53^{\circ} 20^{\prime} 09^{\prime \prime}$ & 134.9 & -7.6 & 40 & 820 & 3 FGL J0212.1+5320 \\
\hline 2FGL J0212.1+5318 & $02^{\mathrm{h}} 12^{\mathrm{m}} 09^{\mathrm{s}}$ & $+53^{\circ} 18^{\prime} 18^{\prime \prime}$ & 134.9 & -7.7 & 25 & 350 & 3 FGL J0212.1+5320 \\
\hline 2FGL J0212.1+5318 & $02^{\mathrm{h}} 12^{\mathrm{m}} 09^{\mathrm{s}}$ & $+53^{\circ} 18^{\prime} 20^{\prime \prime}$ & 134.9 & -7.7 & 38 & 820 & 3FGL J0212.1+5320 \\
\hline P7R40312 * & $02^{\mathrm{h}} 12^{\mathrm{m}} 10^{\mathrm{s}}$ & $+53^{\circ} 19^{\prime} 59^{\prime \prime}$ & 134.9 & -7.6 & 45 & 820 & 3 FGL J0212.1+5320 \\
\hline 1FGL J0212.3+5319 & $02^{\mathrm{h}} 12^{\mathrm{m}} 11^{\mathrm{s}}$ & $+53^{\circ} 20^{\prime} 04^{\prime \prime}$ & 134.9 & -7.6 & 45 & 820 & None \\
\hline 1FGL J0212.3+5319 & $02^{\mathrm{h}} 12^{\mathrm{m}} 11^{\mathrm{s}}$ & $+53^{\circ} 20^{\prime} 04^{\prime \prime}$ & 134.9 & -7.6 & 5 & 820 & None \\
\hline 2FGL J0221.2+2516 & $02^{\mathrm{h}} 21^{\mathrm{m}} 16^{\mathrm{s}}$ & $+25^{\circ} 16^{\prime} 15^{\prime \prime}$ & 147.3 & -33.3 & 42 & 820 & 3 FGL J0221.2+2518 \\
\hline 2FGL J0221.2+2516 & $02^{\mathrm{h}} 21^{\mathrm{m}} 16^{\mathrm{s}}$ & $+25^{\circ} 16^{\prime} 15^{\prime \prime}$ & 147.3 & -33.3 & 5 & 820 & 3 FGL J0221.2+2518 \\
\hline P7R40363 * & $02^{\mathrm{h}} 28^{\mathrm{m}} 01^{\mathrm{s}}$ & $+22^{\circ} 48^{\prime} 01^{\prime \prime}$ & 150.3 & -34.9 & 58 & 350 & 3 FGL J0228.0+2248 \\
\hline P7R40378 & $02^{\mathrm{h}} 32^{\mathrm{m}} 55^{\mathrm{s}}$ & $+26^{\circ} 06^{\prime} 12^{\prime \prime}$ & 149.7 & -31.4 & 45 & 820 & 3FGL J0232.9+2606 \\
\hline P7R40389 * & $02^{\mathrm{h}} 38^{\mathrm{m}} 00^{\mathrm{s}}$ & $+52^{\circ} 37^{\prime} 46^{\prime \prime}$ & 138.8 & -6.9 & 45 & 820 & None \\
\hline P7R40422 * & $02^{\mathrm{h}} 51^{\mathrm{m}} 06^{\mathrm{s}}$ & $+26^{\circ} 03^{\prime} 42^{\prime \prime}$ & 153.9 & -29.5 & 42 & 820 & 3 FGL J0251.1+2603 \\
\hline 1FGL J0258.0+2033 & $02^{\mathrm{h}} 57^{\mathrm{m}} 40^{\mathrm{s}}$ & $+20^{\circ} 25^{\prime} 40^{\prime \prime}$ & 158.9 & -33.5 & 45 & 820 & None \\
\hline 1FGL J0258.0+2033 & $02^{\mathrm{h}} 57^{\mathrm{m}} 40^{\mathrm{s}}$ & $+20^{\circ} 25^{\prime} 40^{\prime \prime}$ & 158.9 & -33.5 & 5 & 820 & None \\
\hline P7R40452 & $03^{\mathrm{h}} 04^{\mathrm{m}} 41^{\mathrm{s}}$ & $-06^{\circ} 04^{\prime} 20^{\prime \prime}$ & 185.4 & -52.0 & 53 & 820 & None \\
\hline P7R40452 & $03^{\mathrm{h}} 04^{\mathrm{m}} 41^{\mathrm{s}}$ & $-06^{\circ} 04^{\prime} 20^{\prime \prime}$ & 185.4 & -52.0 & 53 & 820 & None \\
\hline P7R40452 & $03^{\mathrm{h}} 04^{\mathrm{m}} 41^{\mathrm{s}}$ & $-06^{\circ} 04^{\prime} 21^{\prime \prime}$ & 185.4 & -52.0 & 45 & 820 & None \\
\hline 1FGL J0311.3-0922 & $03^{\mathrm{h}} 11^{\mathrm{m}} 21^{\mathrm{s}}$ & $-09^{\circ} 24^{\prime} 03^{\prime \prime}$ & 191.4 & -52.6 & 5 & 820 & None \\
\hline
\end{tabular}


Table 5.6-Continued

\begin{tabular}{|c|c|c|c|c|c|c|c|}
\hline Source & $\begin{array}{c}\text { R.A. } \\
\text { J2000.0 }\end{array}$ & $\begin{array}{c}\text { Decl. } \\
\text { J2000.0 }\end{array}$ & $\begin{array}{c}l \\
\operatorname{deg}\end{array}$ & $\begin{array}{c}b \\
\operatorname{deg}\end{array}$ & $\begin{array}{l}\text { Integra- } \\
\text { tion Time } \\
\text { minutes }\end{array}$ & $\begin{array}{c}\text { Observing } \\
\text { Freq } \\
\mathrm{MHz}\end{array}$ & 3FGL name \\
\hline P7R40463 * & $03^{\mathrm{h}} 12^{\mathrm{m}} 09^{\mathrm{s}}$ & $-09^{\circ} 21^{\prime} 33^{\prime \prime}$ & 191.5 & -52.4 & 28 & 820 & 3FGL J0312.1-0921 \\
\hline P72Y0468 & $03^{\mathrm{h}} 12^{\mathrm{m}} 57^{\mathrm{s}}$ & $-22^{\circ} 22^{\prime} 29^{\prime \prime}$ & 212.4 & -57.6 & 28 & 820 & 3FGL J0312.7-2222 \\
\hline 1FGL J0318.1+0254 & $03^{\mathrm{h}} 17^{\mathrm{m}} 54^{\mathrm{s}}$ & $+02^{\circ} 56^{\prime} 01^{\prime \prime}$ & 178.3 & -43.6 & 45 & 820 & None \\
\hline 1FGL J0318.1+0254 & $03^{\mathrm{h}} 17^{\mathrm{m}} 54^{\mathrm{s}}$ & $+02^{\circ} 56^{\prime} 01^{\prime \prime}$ & 178.3 & -43.6 & 5 & 820 & None \\
\hline 2FGL J0318.0+0255 & $03^{\mathrm{h}} 18^{\mathrm{m}} 01^{\mathrm{s}}$ & $+02^{\circ} 55^{\prime} 36^{\prime \prime}$ & 178.4 & -43.6 & 6 & 820 & 3FGL J0318.1+0252 \\
\hline 2FGL J0318.0+0255 & $03^{\mathrm{h}} 18^{\mathrm{m}} 01^{\mathrm{s}}$ & $+02^{\circ} 55^{\prime} 37^{\prime \prime}$ & 178.4 & -43.6 & 50 & 820 & 3FGL J0318.1+0252 \\
\hline P7R40505 & $03^{\mathrm{h}} 30^{\mathrm{m}} 39^{\mathrm{s}}$ & $+04^{\circ} 37^{\prime} 44^{\prime \prime}$ & 179.5 & -40.1 & 37 & 820 & 3FGL J0330.6+0437 \\
\hline P7R40509 & $03^{\mathrm{h}} 32^{\mathrm{m}} 00^{\mathrm{s}}$ & $+63^{\circ} 08^{\prime} 52^{\prime \prime}$ & 139.9 & 5.7 & 45 & 820 & 3FGL J0332.0+6308 \\
\hline $24 \mathrm{M} 0388$ & $03^{\mathrm{h}} 32^{\mathrm{m}} 31^{\mathrm{s}}$ & $-11^{\circ} 18^{\prime} 00^{\prime \prime}$ & 198.2 & -49.0 & 45 & 820 & None \\
\hline $24 \mathrm{M} 0388$ & $03^{\mathrm{h}} 32^{\mathrm{m}} 31^{\mathrm{s}}$ & $-11^{\circ} 18^{\prime} 00^{\prime \prime}$ & 198.2 & -49.0 & 5 & 820 & None \\
\hline P7R40512* & $03^{\mathrm{h}} 33^{\mathrm{m}} 28^{\mathrm{s}}$ & $+40^{\circ} 03^{\prime} 05^{\prime \prime}$ & 153.6 & -13.0 & 45 & 820 & 3FGL J0333.4+4003 \\
\hline P72Y0524 & $03^{\mathrm{h}} 34^{\mathrm{m}} 34^{\mathrm{s}}$ & $+78^{\circ} 47^{\prime} 21^{\prime \prime}$ & 130.6 & 18.4 & 50 & 820 & None \\
\hline 2FGL J0336.0+7504 & $03^{\mathrm{h}} 36^{\mathrm{m}} 00^{\mathrm{s}}$ & $+75^{\circ} 04^{\prime} 45^{\prime \prime}$ & 133.1 & 15.5 & 50 & 820 & 3FGL J0336.1+7500 \\
\hline P7R40522 * & $03^{\mathrm{h}} 36^{\mathrm{m}} 08^{\mathrm{s}}$ & $+75^{\circ} 00^{\prime} 38^{\prime \prime}$ & 133.1 & 15.5 & 45 & 820 & 3FGL J0336.1+7500 \\
\hline $24 \mathrm{M} 0400$ & $03^{\mathrm{h}} 36^{\mathrm{m}} 55^{\mathrm{s}}$ & $+32^{\circ} 05^{\prime} 24^{\prime \prime}$ & 159.2 & -18.9 & 45 & 820 & 3FGL J0336.5+3210 \\
\hline $24 \mathrm{M} 0400$ & $03^{\mathrm{h}} 36^{\mathrm{m}} 55^{\mathrm{s}}$ & $+32^{\circ} 05^{\prime} 24^{\prime \prime}$ & 159.2 & -18.9 & 5 & 820 & 3FGL J0336.5+3210 \\
\hline P7R40535 & $03^{\mathrm{h}} 40^{\mathrm{m}} 25^{\mathrm{s}}$ & $-02^{\circ} 22^{\prime} 26^{\prime \prime}$ & 188.8 & -42.6 & 40 & 350 & None \\
\hline P72Y0550 & $03^{\mathrm{h}} 45^{\mathrm{m}} 37^{\mathrm{s}}$ & $+32^{\circ} 39^{\prime} 31^{\prime \prime}$ & 160.3 & -17.3 & 36 & 820 & 3FGL J0345.3+3236 \\
\hline $24 \mathrm{M} 0444$ & $03^{\mathrm{h}} 59^{\mathrm{m}} 00^{\mathrm{s}}$ & $+60^{\circ} 02^{\prime} 24^{\prime \prime}$ & 144.4 & 5.2 & 45 & 820 & 3FGL J0358.8+6002 \\
\hline $24 \mathrm{M} 0444$ & $03^{\mathrm{h}} 59^{\mathrm{m}} 00^{\mathrm{s}}$ & $+60^{\circ} 02^{\prime} 24^{\prime \prime}$ & 144.4 & 5.2 & 5 & 820 & 3FGL J0358.8+6002 \\
\hline $24 \mathrm{M} 0453$ & $04^{\mathrm{h}} 01^{\mathrm{m}} 36^{\mathrm{s}}$ & $-20^{\circ} 36^{\prime} 00^{\prime \prime}$ & 215.0 & -46.3 & 20 & 820 & None \\
\hline $24 \mathrm{M} 0453$ & $04^{\mathrm{h}} 01^{\mathrm{m}} 36^{\mathrm{s}}$ & $-20^{\circ} 36^{\prime} 00^{\prime \prime}$ & 215.0 & -46.3 & 5 & 820 & None \\
\hline P7R40591A * & $04^{\mathrm{h}} 12^{\mathrm{m}} 05^{\mathrm{s}}$ & $+02^{\circ} 29^{\prime} 44^{\prime \prime}$ & 189.6 & -33.4 & 40 & 350 & 3FGL J0412.0+0229 \\
\hline P7R40602 * & $04^{\mathrm{h}} 19^{\mathrm{m}} 06^{\mathrm{s}}$ & $+66^{\circ} 36^{\prime} 19^{\prime \prime}$ & 141.5 & 11.6 & 45 & 820 & 3FGL J0419.1+6636 \\
\hline 2FGL J0423.4+5612 & $04^{\mathrm{h}} 23^{\mathrm{m}} 26^{\mathrm{s}}$ & $+56^{\circ} 12^{\prime} 21^{\prime \prime}$ & 149.4 & 4.6 & 50 & 820 & None \\
\hline 1FGL J0426.5+5437 & $04^{\mathrm{h}} 26^{\mathrm{m}} 41^{\mathrm{s}}$ & $+54^{\circ} 35^{\prime} 45^{\prime \prime}$ & 150.9 & 3.9 & 45 & 820 & None \\
\hline 1FGL J0426.5+5437 & $04^{\mathrm{h}} 26^{\mathrm{m}} 41^{\mathrm{s}}$ & $+54^{\circ} 35^{\prime} 45^{\prime \prime}$ & 150.9 & 3.9 & 5 & 820 & None \\
\hline 2FGL J0426.7+5434 & $04^{\mathrm{h}} 26^{\mathrm{m}} 45^{\mathrm{s}}$ & $+54^{\circ} 34^{\prime} 51^{\prime \prime}$ & 150.9 & 3.9 & 50 & 820 & 3FGL J0426.7+5437 \\
\hline 2FGL J0428.0-3845 & $04^{\mathrm{h}} 28^{\mathrm{m}} 03^{\mathrm{s}}$ & $-38^{\circ} 45^{\prime} 41^{\prime \prime}$ & 241.8 & -43.8 & 39 & 820 & None \\
\hline 2FGL J0428.0-3845 & $04^{\mathrm{h}} 28^{\mathrm{m}} 03^{\mathrm{s}}$ & $-38^{\circ} 45^{\prime} 41^{\prime \prime}$ & 241.8 & -43.8 & 5 & 820 & None \\
\hline 2 FGL J0430.2+3508c & $04^{\mathrm{h}} 30^{\mathrm{m}} 15^{\mathrm{s}}$ & $+35^{\circ} 08^{\prime} 57^{\prime \prime}$ & 165.5 & -9.1 & 50 & 820 & None \\
\hline 2FGL J0430.2+3508c & $04^{\mathrm{h}} 30^{\mathrm{m}} 15^{\mathrm{s}}$ & $+35^{\circ} 08^{\prime} 57^{\prime \prime}$ & 165.5 & -9.1 & 5 & 820 & None \\
\hline P72Y0641 & $04^{\mathrm{h}} 32^{\mathrm{m}} 14^{\mathrm{s}}$ & $+55^{\circ} 55^{\prime} 41^{\prime \prime}$ & 150.5 & 5.3 & 40 & 820 & None \\
\hline 1FGL J0448.6+1118 & $04^{\mathrm{h}} 48^{\mathrm{m}} 56^{\mathrm{s}}$ & $+11^{\circ} 22^{\prime} 10^{\prime \prime}$ & 187.4 & -20.8 & 5 & 820 & None \\
\hline P72Y0703 & $05^{\mathrm{h}} 00^{\mathrm{m}} 06^{\mathrm{s}}$ & $+52^{\circ} 36^{\prime} 13^{\prime \prime}$ & 155.6 & 6.3 & 45 & 820 & 3FGL J0500.3+5237 \\
\hline P72Y1005 & $05^{\mathrm{h}} 18^{\mathrm{m}} 08^{\mathrm{s}}$ & $+55^{\circ} 04^{\prime} 21^{\prime \prime}$ & 155.2 & 9.9 & 33 & 820 & None \\
\hline 1FGL J0523.5-2529 & $05^{\mathrm{h}} 23^{\mathrm{m}} 27^{\mathrm{s}}$ & $-25^{\circ} 29^{\prime} 47^{\prime \prime}$ & 228.2 & -29.8 & 45 & 820 & None \\
\hline 1FGL J0523.5-2529 & $05^{\mathrm{h}} 23^{\mathrm{m}} 27^{\mathrm{s}}$ & $-25^{\circ} 29^{\prime} 47^{\prime \prime}$ & 228.2 & -29.8 & 5 & 820 & None \\
\hline P7R40759 & $05^{\mathrm{h}} 26^{\mathrm{m}} 26^{\mathrm{s}}$ & $+22^{\circ} 47^{\prime} 54^{\prime \prime}$ & 182.9 & -6.9 & 35 & 820 & 3FGL J0526.4+2247 \\
\hline P72Y0772 & $05^{\mathrm{h}} 26^{\mathrm{m}} 59^{\mathrm{s}}$ & $+66^{\circ} 48^{\prime} 14^{\prime \prime}$ & 145.5 & 17.0 & 45 & 820 & 3FGL J0527.3+6647 \\
\hline $24 \mathrm{M} 0660$ & $05^{\mathrm{h}} 29^{\mathrm{m}} 21^{\mathrm{s}}$ & $+09^{\circ} 38^{\prime} 25^{\prime \prime}$ & 194.6 & -13.4 & 45 & 820 & 3FGL J0529.1+0933 \\
\hline
\end{tabular}


Table 5.6-Continued

\begin{tabular}{|c|c|c|c|c|c|c|c|}
\hline Source & $\begin{array}{c}\text { R.A. } \\
\text { J2000.0 }\end{array}$ & $\begin{array}{c}\text { Decl. } \\
\text { J2000.0 }\end{array}$ & $\begin{array}{c}l \\
\operatorname{deg}\end{array}$ & $\begin{array}{c}b \\
\operatorname{deg}\end{array}$ & $\begin{array}{l}\text { Integra- } \\
\text { tion Time } \\
\text { minutes }\end{array}$ & $\begin{array}{c}\text { Observing } \\
\text { Freq } \\
\mathrm{MHz}\end{array}$ & 3FGL name \\
\hline $24 \mathrm{M} 0660$ & $05^{\mathrm{h}} 29^{\mathrm{m}} 21^{\mathrm{s}}$ & $+09^{\circ} 38^{\prime} 25^{\prime \prime}$ & 194.6 & -13.4 & 5 & 820 & 3FGL J0529.1+0933 \\
\hline 2FGL J0533.9+6759 † & $05^{\mathrm{h}} 33^{\mathrm{m}} 57^{\mathrm{s}}$ & $+67^{\circ} 59^{\prime} 51^{\prime \prime}$ & 144.8 & 18.2 & 41 & 820 & 3FGL J0534.0+6759 \\
\hline 2 FGL J0533.9+6759 ${ }^{\dagger}$ & $05^{\mathrm{h}} 33^{\mathrm{m}} 57^{\mathrm{s}}$ & $+67^{\circ} 59^{\prime} 51^{\prime \prime}$ & 144.8 & 18.2 & 5 & 820 & 3FGL J0534.0+6759 \\
\hline 2FGL J0534.8-0548c & $05^{\mathrm{h}} 34^{\mathrm{m}} 52^{\mathrm{s}}$ & $-05^{\circ} 48^{\prime} 42^{\prime \prime}$ & 209.4 & -19.7 & 50 & 820 & None \\
\hline 2FGL J0534.9-0450c & $05^{\mathrm{h}} 34^{\mathrm{m}} 54^{\mathrm{s}}$ & $-04^{\circ} 50^{\prime} 17^{\prime \prime}$ & 208.4 & -19.2 & 5 & 820 & None \\
\hline 1FGL J0536.2-0607c & $05^{\mathrm{h}} 36^{\mathrm{m}} 05^{\mathrm{s}}$ & $-06^{\circ} 10^{\prime} 04^{\prime \prime}$ & 209.8 & -19.6 & 30 & 820 & None \\
\hline 1FGL J0536.2-0607c & $05^{\mathrm{h}} 36^{\mathrm{m}} 05^{\mathrm{s}}$ & $-06^{\circ} 10^{\prime} 04^{\prime \prime}$ & 209.8 & -19.6 & 5 & 820 & None \\
\hline 2FGL J0538.5-0534c & $05^{\mathrm{h}} 38^{\mathrm{m}} 35^{\mathrm{s}}$ & $-05^{\circ} 34^{\prime} 28^{\prime \prime}$ & 209.6 & -18.7 & 32 & 820 & 3FGL J0539.2-0536 \\
\hline 1FGL J0539.4-0400 & $05^{\mathrm{h}} 40^{\mathrm{m}} 08^{\mathrm{s}}$ & $-04^{\circ} 02^{\prime} 56^{\prime \prime}$ & 208.3 & -17.7 & 45 & 820 & None \\
\hline 1FGL J0539.4-0400 & $05^{\mathrm{h}} 40^{\mathrm{m}} 08^{\mathrm{s}}$ & $-04^{\circ} 02^{\prime} 56^{\prime \prime}$ & 208.3 & -17.7 & 5 & 820 & None \\
\hline 2FGL J0541.8-0203c & $05^{\mathrm{h}} 41^{\mathrm{m}} 48^{\mathrm{s}}$ & $-02^{\circ} 03^{\prime} 38^{\prime \prime}$ & 206.7 & -16.4 & 30 & 350 & None \\
\hline 2FGL J0541.8-0203c & $05^{\mathrm{h}} 41^{\mathrm{m}} 49^{\mathrm{s}}$ & $-02^{\circ} 03^{\prime} 37^{\prime \prime}$ & 206.7 & -16.4 & 50 & 820 & None \\
\hline P72Y0835 & $05^{\mathrm{h}} 41^{\mathrm{m}} 50^{\mathrm{s}}$ & $-01^{\circ} 49^{\prime} 55^{\prime \prime}$ & 206.5 & -16.3 & 40 & 350 & None \\
\hline P72Y0835 & $05^{\mathrm{h}} 41^{\mathrm{m}} 50^{\mathrm{s}}$ & $-01^{\circ} 49^{\prime} 55^{\prime \prime}$ & 206.5 & -16.3 & 60 & 350 & None \\
\hline P72Y0835 & $05^{\mathrm{h}} 41^{\mathrm{m}} 50^{\mathrm{s}}$ & $-01^{\circ} 49^{\prime} 56^{\prime \prime}$ & 206.5 & -16.3 & 45 & 820 & None \\
\hline 2FGL J0543.2-0120c & $05^{\mathrm{h}} 43^{\mathrm{m}} 14^{\mathrm{s}}$ & $-01^{\circ} 20^{\prime} 08^{\prime \prime}$ & 206.2 & -15.8 & 38 & 820 & None \\
\hline 2FGL J0543.2-0120c & $05^{\mathrm{h}} 43^{\mathrm{m}} 14^{\mathrm{s}}$ & $-01^{\circ} 20^{\prime} 08^{\prime \prime}$ & 206.2 & -15.8 & 5 & 820 & None \\
\hline 2FGL J0545.6+6018 & $05^{\mathrm{h}} 45^{\mathrm{m}} 38^{\mathrm{s}}$ & $+60^{\circ} 18^{\prime} 51^{\prime \prime}$ & 152.5 & 15.7 & 35 & 350 & 3FGL J0545.6+6019 \\
\hline 1FGL J0547.0+0020c & $05^{\mathrm{h}} 47^{\mathrm{m}} 03^{\mathrm{s}}$ & $-04^{\circ} 02^{\prime} 56^{\prime \prime}$ & 209.2 & -16.2 & 45 & 820 & None \\
\hline 1FGL J0547.0+0020c & $05^{\mathrm{h}} 47^{\mathrm{m}} 03^{\mathrm{s}}$ & $-04^{\circ} 02^{\prime} 56^{\prime \prime}$ & 209.2 & -16.2 & 5 & 820 & None \\
\hline 2FGL J0547.1+0020c & $05^{\mathrm{h}} 47^{\mathrm{m}} 11^{\mathrm{s}}$ & $-02^{\circ} 03^{\prime} 38^{\prime \prime}$ & 207.3 & -15.2 & 26 & 350 & 3FGL J0546.4+0031c \\
\hline 2FGL J0547.5-0141c & $05^{\mathrm{h}} 47^{\mathrm{m}} 31^{\mathrm{s}}$ & $-01^{\circ} 41^{\prime} 11^{\prime \prime}$ & 207.0 & -15.0 & 30 & 350 & None \\
\hline 2FGL J0547.5-0141c & $05^{\mathrm{h}} 47^{\mathrm{m}} 31^{\mathrm{s}}$ & $-01^{\circ} 41^{\prime} 11^{\prime \prime}$ & 207.0 & -15.0 & 48 & 820 & None \\
\hline P72Y0859 & $05^{\mathrm{h}} 53^{\mathrm{m}} 26^{\mathrm{s}}$ & $-20^{\circ} 35^{\prime} 05^{\prime \prime}$ & 225.7 & -21.7 & 40 & 350 & 3FGL J0553.5-2036 \\
\hline 1FGL J0553.9+3105 & $05^{\mathrm{h}} 53^{\mathrm{m}} 56^{\mathrm{s}}$ & $+31^{\circ} 07^{\prime} 07^{\prime \prime}$ & 179.1 & 2.7 & 5 & 820 & None \\
\hline 1FGL J0553.9+3105 & $05^{\mathrm{h}} 53^{\mathrm{m}} 56^{\mathrm{s}}$ & $+31^{\circ} 07^{\prime} 07^{\prime \prime}$ & 179.1 & 2.7 & 5 & 820 & None \\
\hline P72Y0862 & $05^{\mathrm{h}} 54^{\mathrm{m}} 40^{\mathrm{s}}$ & $+03^{\circ} 04^{\prime} 56^{\prime \prime}$ & 203.6 & -11.2 & 45 & 350 & None \\
\hline 1FGL J0600.5-2006 & $06^{\mathrm{h}} 01^{\mathrm{m}} 01^{\mathrm{s}}$ & $-19^{\circ} 54^{\prime} 00^{\prime \prime}$ & 225.8 & -19.7 & 5 & 820 & None \\
\hline 2 FGL J0605.3+3758 ${ }^{\dagger}$ & $06^{\mathrm{h}} 05^{\mathrm{m}} 23^{\mathrm{s}}$ & $+37^{\circ} 58^{\prime} 45^{\prime \prime}$ & 174.2 & 8.1 & 11 & 820 & 3FGL J0605.0+3756 \\
\hline 2 FGL J0605.3+3758 ${ }^{\dagger}$ & $06^{\mathrm{h}} 05^{\mathrm{m}} 23^{\mathrm{s}}$ & $+37^{\circ} 58^{\prime} 45^{\prime \prime}$ & 174.2 & 8.1 & 12 & 820 & 3FGL J0605.0+3756 \\
\hline 2 FGL J0605.3+3758 ${ }^{\dagger}$ & $06^{\mathrm{h}} 05^{\mathrm{m}} 23^{\mathrm{s}}$ & $+37^{\circ} 58^{\prime} 45^{\prime \prime}$ & 174.2 & 8.1 & 1 & 820 & 3FGL J0605.0+3756 \\
\hline 2 FGL J0605.3+3758 ${ }^{\dagger}$ & $06^{\mathrm{h}} 05^{\mathrm{m}} 23^{\mathrm{s}}$ & $+37^{\circ} 58^{\prime} 46^{\prime \prime}$ & 174.2 & 8.1 & 11 & 820 & 3FGL J0605.0+3756 \\
\hline 2 FGL J0605.3+3758 ${ }^{\dagger}$ & $06^{\mathrm{h}} 05^{\mathrm{m}} 23^{\mathrm{s}}$ & $+37^{\circ} 58^{\prime} 46^{\prime \prime}$ & 174.2 & 8.1 & 12 & 820 & 3FGL J0605.0+3756 \\
\hline 2 FGL J0605.3+3758 † & $06^{\mathrm{h}} 05^{\mathrm{m}} 23^{\mathrm{s}}$ & $+37^{\circ} 58^{\prime} 46^{\prime \prime}$ & 174.2 & 8.1 & 50 & 820 & 3FGL J0605.0+3756 \\
\hline 1FGL J0605.3+3800 & $06^{\mathrm{h}} 05^{\mathrm{m}} 24^{\mathrm{s}}$ & $+37^{\circ} 58^{\prime} 06^{\prime \prime}$ & 174.2 & 8.1 & 5 & 820 & None \\
\hline 1FGL J0605.3+3800 & $06^{\mathrm{h}} 05^{\mathrm{m}} 24^{\mathrm{s}}$ & $+37^{\circ} 58^{\prime} 07^{\prime \prime}$ & 174.2 & 8.1 & 5 & 820 & None \\
\hline 2 FGL J0605.3+3758 ${ }^{\dagger}$ & $06^{\mathrm{h}} 05^{\mathrm{m}} 24^{\mathrm{s}}$ & $+37^{\circ} 58^{\prime} 51^{\prime \prime}$ & 174.2 & 8.1 & 10 & 820 & 3FGL J0605.0+3756 \\
\hline 1FGL J0608.1-0630c & $06^{\mathrm{h}} 08^{\mathrm{m}} 17^{\mathrm{s}}$ & $-06^{\circ} 17^{\prime} 02^{\prime \prime}$ & 213.7 & -12.4 & 45 & 820 & None \\
\hline P72Y0892 & $06^{\mathrm{h}} 08^{\mathrm{m}} 31^{\mathrm{s}}$ & $-06^{\circ} 10^{\prime} 02^{\prime \prime}$ & 213.6 & -12.3 & 40 & 350 & None \\
\hline P7R40880 * & $06^{\mathrm{h}} 11^{\mathrm{m}} 43^{\mathrm{s}}$ & $+27^{\circ} 59^{\prime} 52^{\prime \prime}$ & 183.7 & 4.5 & 45 & 820 & 3FGL J0611.7+2759 \\
\hline 2FGL J0616.6+2425 & $06^{\mathrm{h}} 16^{\mathrm{m}} 36^{\mathrm{s}}$ & $+24^{\circ} 25^{\prime} 15^{\prime \prime}$ & 187.4 & 3.8 & 50 & 820 & None \\
\hline
\end{tabular}


Table 5.6-Continued

\begin{tabular}{|c|c|c|c|c|c|c|c|}
\hline Source & $\begin{array}{c}\text { R.A. } \\
\text { J2000.0 }\end{array}$ & $\begin{array}{c}\text { Decl. } \\
\text { J2000.0 }\end{array}$ & $\begin{array}{c}l \\
\operatorname{deg}\end{array}$ & $\begin{array}{c}b \\
\operatorname{deg}\end{array}$ & $\begin{array}{l}\text { Integra- } \\
\text { tion Time } \\
\text { minutes }\end{array}$ & $\begin{array}{c}\text { Observing } \\
\text { Freq } \\
\mathrm{MHz}\end{array}$ & 3FGL name \\
\hline 2FGL J0616.6+2425 & $06^{\mathrm{h}} 16^{\mathrm{m}} 36^{\mathrm{s}}$ & $+24^{\circ} 25^{\prime} 15^{\prime \prime}$ & 187.4 & 3.8 & 5 & 820 & None \\
\hline 1FGL J0621.5+2508 & $06^{\mathrm{h}} 21^{\mathrm{m}} 13^{\mathrm{s}}$ & $+25^{\circ} 10^{\prime} 31^{\prime \prime}$ & 187.2 & 5.1 & 45 & 820 & None \\
\hline 1FGL J0621.5+2508 & $06^{\mathrm{h}} 21^{\mathrm{m}} 13^{\mathrm{s}}$ & $+25^{\circ} 10^{\prime} 31^{\prime \prime}$ & 187.2 & 5.1 & 5 & 820 & None \\
\hline 2FGL J0621.2+2508 & $06^{\mathrm{h}} 21^{\mathrm{m}} 15^{\mathrm{s}}$ & $+25^{\circ} 08^{\prime} 20^{\prime \prime}$ & 187.2 & 5.0 & 50 & 820 & 3FGL J0621.0+2514 \\
\hline 2FGL J0621.2+2508 & $06^{\mathrm{h}} 21^{\mathrm{m}} 15^{\mathrm{s}}$ & $+25^{\circ} 08^{\prime} 20^{\prime \prime}$ & 187.2 & 5.0 & 5 & 820 & 3FGL J0621.0+2514 \\
\hline 1FGL J0622.2+3751 & $06^{\mathrm{h}} 21^{\mathrm{m}} 59^{\mathrm{s}}$ & $+37^{\circ} 51^{\prime} 36^{\prime \prime}$ & 175.8 & 10.9 & 5 & 820 & None \\
\hline 1FGL J0622.2+3751 & $06^{\mathrm{h}} 21^{\mathrm{m}} 59^{\mathrm{s}}$ & $+37^{\circ} 51^{\prime} 36^{\prime \prime}$ & 175.8 & 10.9 & 5 & 820 & None \\
\hline $24 \mathrm{M} 0825$ & $06^{\mathrm{h}} 32^{\mathrm{m}} 35^{\mathrm{s}}$ & $+04^{\circ} 27^{\prime} 36^{\prime \prime}$ & 206.8 & -2.1 & 45 & 820 & None \\
\hline $24 \mathrm{M} 0825$ & $06^{\mathrm{h}} 32^{\mathrm{m}} 35^{\mathrm{s}}$ & $+04^{\circ} 27^{\prime} 36^{\prime \prime}$ & 206.8 & -2.1 & 5 & 820 & None \\
\hline 1FGL J0643.2+0859 & $06^{\mathrm{h}} 43^{\mathrm{m}} 14^{\mathrm{s}}$ & $+08^{\circ} 58^{\prime} 40^{\prime \prime}$ & 204.0 & 2.3 & 45 & 820 & None \\
\hline 1FGL J0643.2+0859 & $06^{\mathrm{h}} 43^{\mathrm{m}} 14^{\mathrm{s}}$ & $+08^{\circ} 58^{\prime} 40^{\prime \prime}$ & 204.0 & 2.3 & 5 & 820 & None \\
\hline P7R41060 * & $07^{\mathrm{h}} 40^{\mathrm{m}} 55^{\mathrm{s}}$ & $+66^{\circ} 22^{\prime} 30^{\prime \prime}$ & 149.7 & 29.6 & 33 & 820 & 3FGL J0740.8+6621 \\
\hline P72Y1109 & $07^{\mathrm{h}} 44^{\mathrm{m}} 10^{\mathrm{s}}$ & $+17^{\circ} 18^{\prime} 42^{\prime \prime}$ & 202.8 & 19.2 & 35 & 350 & 3FGL J0744.3+1715 \\
\hline P72Y1109 & $07^{\mathrm{h}} 44^{\mathrm{m}} 10^{\mathrm{s}}$ & $+17^{\circ} 18^{\prime} 42^{\prime \prime}$ & 202.8 & 19.2 & 35 & 350 & 3FGL J0744.3+1715 \\
\hline P7R41204 * & $08^{\mathrm{h}} 38^{\mathrm{m}} 47^{\mathrm{s}}$ & $-28^{\circ} 28^{\prime} 41^{\prime \prime}$ & 250.6 & 7.8 & 30 & 820 & 3FGL J0838.8-2829 \\
\hline 2FGL J0838.8-2828 & $08^{\mathrm{h}} 38^{\mathrm{m}} 51^{\mathrm{s}}$ & $-28^{\circ} 28^{\prime} 32^{\prime \prime}$ & 250.6 & 7.8 & 5 & 820 & 3FGL J0838.8-2829 \\
\hline 2FGL J0841.3-3556 & $08^{\mathrm{h}} 41^{\mathrm{m}} 21^{\mathrm{s}}$ & $-35^{\circ} 56^{\prime} 21^{\prime \prime}$ & 256.9 & 3.7 & 50 & 820 & 3FGL J0841.3-3554 \\
\hline 2FGL J0841.3-3556 & $08^{\mathrm{h}} 41^{\mathrm{m}} 21^{\mathrm{s}}$ & $-35^{\circ} 56^{\prime} 21^{\prime \prime}$ & 256.9 & 3.7 & 5 & 820 & 3FGL J0841.3-3554 \\
\hline 1FGL J0843.4+6718 & $08^{\mathrm{h}} 43^{\mathrm{m}} 38^{\mathrm{s}}$ & $+67^{\circ} 15^{\prime} 56^{\prime \prime}$ & 147.7 & 35.6 & 34 & 820 & None \\
\hline 1FGL J0843.4+6718 & $08^{\mathrm{h}} 43^{\mathrm{m}} 38^{\mathrm{s}}$ & $+67^{\circ} 15^{\prime} 56^{\prime \prime}$ & 147.7 & 35.6 & 5 & 820 & None \\
\hline P72Y1279 & $08^{\mathrm{h}} 49^{\mathrm{m}} 18^{\mathrm{s}}$ & $-29^{\circ} 13^{\prime} 21^{\prime \prime}$ & 252.6 & 9.2 & 30 & 820 & 3FGL J0849.5-2912 \\
\hline P72Y1280 & $08^{\mathrm{h}} 49^{\mathrm{m}} 47^{\mathrm{s}}$ & $+51^{\circ} 09^{\prime} 47^{\prime \prime}$ & 167.6 & 39.1 & 61 & 820 & 3FGL J0849.9+5108 \\
\hline 24M1109.2 & $08^{\mathrm{h}} 54^{\mathrm{m}} 52^{\mathrm{s}}$ & $+71^{\circ} 51^{\prime} 00^{\prime \prime}$ & 142.0 & 35.1 & 45 & 820 & 3FGL J0855.4+7142 \\
\hline 24M1109.2 & $08^{\mathrm{h}} 54^{\mathrm{m}} 52^{\mathrm{s}}$ & $+71^{\circ} 51^{\prime} 00^{\prime \prime}$ & 142.0 & 35.1 & 5 & 820 & 3FGL J0855.4+7142 \\
\hline 1FGL J0856.6+2103 & $08^{\mathrm{h}} 56^{\mathrm{m}} 32^{\mathrm{s}}$ & $+20^{\circ} 59^{\prime} 04^{\prime \prime}$ & 206.0 & 36.5 & 45 & 820 & None \\
\hline P72Y1389 & $09^{\mathrm{h}} 35^{\mathrm{m}} 45^{\mathrm{s}}$ & $+09^{\circ} 02^{\prime} 21^{\prime \prime}$ & 224.8 & 40.5 & 35 & 350 & 3FGL J0935.2+0903 \\
\hline P72Y1389 & $09^{\mathrm{h}} 35^{\mathrm{m}} 45^{\mathrm{s}}$ & $+09^{\circ} 02^{\prime} 22^{\prime \prime}$ & 224.8 & 40.5 & 28 & 350 & 3FGL J0935.2+0903 \\
\hline P72Y1389 & $09^{\mathrm{h}} 35^{\mathrm{m}} 45^{\mathrm{s}}$ & $+09^{\circ} 02^{\prime} 22^{\prime \prime}$ & 224.8 & 40.5 & 34 & 820 & 3FGL J0935.2+0903 \\
\hline 2FGL J0953.6-1504 & $09^{\mathrm{h}} 53^{\mathrm{m}} 35^{\mathrm{s}}$ & $-15^{\circ} 08^{\prime} 32^{\prime \prime}$ & 251.9 & 29.6 & 27 & 350 & 3FGL J0953.7-1510 \\
\hline P72Y1445 & $10^{\mathrm{h}} 03^{\mathrm{m}} 46^{\mathrm{s}}$ & $+26^{\circ} 10^{\prime} 32^{\prime \prime}$ & 204.6 & 52.7 & 35 & 350 & 3FGL J1003.6+2608 \\
\hline P72Y1445 & $10^{\mathrm{h}} 03^{\mathrm{m}} 46^{\mathrm{s}}$ & $+26^{\circ} 10^{\prime} 32^{\prime \prime}$ & 204.6 & 52.7 & 35 & 350 & 3 FGL J1003.6+2608 \\
\hline P72Y1445 & $10^{\mathrm{h}} 03^{\mathrm{m}} 46^{\mathrm{s}}$ & $+26^{\circ} 10^{\prime} 32^{\prime \prime}$ & 204.6 & 52.7 & 35 & 350 & 3FGL J1003.6+2608 \\
\hline P7R41464 & $10^{\mathrm{h}} 27^{\mathrm{m}} 51^{\mathrm{s}}$ & $+82^{\circ} 53^{\prime} 52^{\prime \prime}$ & 127.9 & 32.8 & 45 & 820 & 3FGL J1027.8+8253 \\
\hline P72Y1529 & $10^{\mathrm{h}} 32^{\mathrm{m}} 07^{\mathrm{s}}$ & $+66^{\circ} 25^{\prime} 24^{\prime \prime}$ & 141.9 & 45.2 & 55 & 820 & 3FGL J1032.5+6623 \\
\hline P72Y1549 & $10^{\mathrm{h}} 40^{\mathrm{m}} 58^{\mathrm{s}}$ & $-12^{\circ} 05^{\prime} 01^{\prime \prime}$ & 259.8 & 39.6 & 35 & 350 & 3FGL J1040.9-1205 \\
\hline P72Y1549 & $10^{\mathrm{h}} 40^{\mathrm{m}} 58^{\mathrm{s}}$ & $-12^{\circ} 05^{\prime} 01^{\prime \prime}$ & 259.8 & 39.6 & 35 & 350 & 3FGL J1040.9-1205 \\
\hline P72Y1549 & $10^{\mathrm{h}} 40^{\mathrm{m}} 58^{\mathrm{s}}$ & $-12^{\circ} 05^{\prime} 01^{\prime \prime}$ & 259.8 & 39.6 & 35 & 350 & 3FGL J1040.9-1205 \\
\hline SEED3Y-103 & $10^{\mathrm{h}} 50^{\mathrm{m}} 30^{\mathrm{s}}$ & $+04^{\circ} 36^{\prime} 01^{\prime \prime}$ & 245.6 & 53.4 & 35 & 350 & 3FGL J1050.4+0435 \\
\hline SEED3Y-103 & $10^{\mathrm{h}} 50^{\mathrm{m}} 30^{\mathrm{s}}$ & $+04^{\circ} 36^{\prime} 01^{\prime \prime}$ & 245.6 & 53.4 & 35 & 350 & 3FGL J1050.4+0435 \\
\hline P72Y1633 & $11^{\mathrm{h}} 06^{\mathrm{m}} 36^{\mathrm{s}}$ & $-17^{\circ} 43^{\prime} 24^{\prime \prime}$ & 270.4 & 38.4 & 35 & 350 & 3FGL J1106.6-1744 \\
\hline SEED3Y-110 & $11^{\mathrm{h}} 18^{\mathrm{m}} 25^{\mathrm{s}}$ & $-04^{\circ} 14^{\prime} 13^{\prime \prime}$ & 263.8 & 51.4 & 35 & 350 & 3FGL J1118.2-0411 \\
\hline
\end{tabular}


Table 5.6-Continued

\begin{tabular}{|c|c|c|c|c|c|c|c|}
\hline Source & $\begin{array}{c}\text { R.A. } \\
\text { J2000.0 }\end{array}$ & $\begin{array}{c}\text { Decl. } \\
\text { J2000.0 }\end{array}$ & $\begin{array}{c}l \\
\operatorname{deg}\end{array}$ & $\begin{array}{c}b \\
\operatorname{deg}\end{array}$ & $\begin{array}{c}\text { Integra- } \\
\text { tion Time } \\
\text { minutes }\end{array}$ & $\begin{array}{c}\text { Observing } \\
\text { Freq } \\
\mathrm{MHz}\end{array}$ & 3FGL name \\
\hline SEED3Y-110 & $11^{\mathrm{h}} 18^{\mathrm{m}} 25^{\mathrm{s}}$ & $-04^{\circ} 14^{\prime} 13^{\prime \prime}$ & 263.8 & 51.4 & 35 & 350 & 3FGL J1118.2-0411 \\
\hline P7R41624 & $11^{\mathrm{h}} 19^{\mathrm{m}} 56^{\mathrm{s}}$ & $-22^{\circ} 04^{\prime} 02^{\prime \prime}$ & 276.5 & 36.1 & 49 & 820 & 3FGL J1119.9-2204 \\
\hline 2FGL J1120.0-2204 & $11^{\mathrm{h}} 20^{\mathrm{m}} 00^{\mathrm{s}}$ & $-22^{\circ} 04^{\prime} 50^{\prime \prime}$ & 276.5 & 36.1 & 44 & 820 & 3FGL J1119.9-2204 \\
\hline 2FGL J1120.0-2204 & $11^{\mathrm{h}} 20^{\mathrm{m}} 00^{\mathrm{s}}$ & $-22^{\circ} 04^{\prime} 50^{\prime \prime}$ & 276.5 & 36.1 & 5 & 820 & 3FGL J1119.9-2204 \\
\hline 1FGL J1123.6-2528 & $11^{\mathrm{h}} 23^{\mathrm{m}} 39^{\mathrm{s}}$ & $-25^{\circ} 27^{\prime} 24^{\prime \prime}$ & 279.1 & 33.3 & 5 & 820 & None \\
\hline P72Y1698 & $11^{\mathrm{h}} 26^{\mathrm{m}} 56^{\mathrm{s}}$ & $-18^{\circ} 57^{\prime} 21^{\prime \prime}$ & 276.7 & 39.6 & 50 & 820 & 3FGL J1127.0-1857 \\
\hline P72Y1724 ${ }^{\dagger}$ & $11^{\mathrm{h}} 35^{\mathrm{m}} 35^{\mathrm{s}}$ & $+75^{\circ} 28^{\prime} 10^{\prime \prime}$ & 129.1 & 40.7 & 35 & 350 & 3FGL J1136.1+7523 \\
\hline 1FGL J1142.7+0127 † & $11^{\mathrm{h}} 42^{\mathrm{m}} 52^{\mathrm{s}}$ & $+01^{\circ} 20^{\prime} 54^{\prime \prime}$ & 267.5 & 59.4 & 5 & 820 & None \\
\hline 1FGL J1142.7+0127 † & $11^{\mathrm{h}} 42^{\mathrm{m}} 53^{\mathrm{s}}$ & $+01^{\circ} 20^{\prime} 54^{\prime \prime}$ & 267.5 & 59.4 & 5 & 820 & None \\
\hline P72Y1813 & $12^{\mathrm{h}} 12^{\mathrm{m}} 29^{\mathrm{s}}$ & $+51^{\circ} 28^{\prime} 53^{\prime \prime}$ & 137.1 & 64.6 & 35 & 820 & 3FGL J1212.6+5135 \\
\hline 2FGL J1226.0+2953 & $12^{\mathrm{h}} 26^{\mathrm{m}} 04^{\mathrm{s}}$ & $+29^{\circ} 53^{\prime} 46^{\prime \prime}$ & 185.0 & 83.8 & 50 & 820 & 3FGL J1225.9+2953 \\
\hline 1FGL J1249.8+3706 & $12^{\mathrm{h}} 49^{\mathrm{m}} 42^{\mathrm{s}}$ & $+37^{\circ} 05^{\prime} 13^{\prime \prime}$ & 124.9 & 80.0 & 45 & 820 & None \\
\hline 2FGL J1311.7-3429 & $13^{\mathrm{h}} 11^{\mathrm{m}} 46^{\mathrm{s}}$ & $-34^{\circ} 29^{\prime} 19^{\prime \prime}$ & 307.7 & 28.2 & 5 & 820 & 3FGL J1311.8-3430 \\
\hline 1FGL J1311.7-3429 & $13^{\mathrm{h}} 11^{\mathrm{m}} 48^{\mathrm{s}}$ & $-34^{\circ} 29^{\prime} 48^{\prime \prime}$ & 307.7 & 28.2 & 38 & 820 & None \\
\hline 1FGL J1311.7-3429 & $13^{\mathrm{h}} 11^{\mathrm{m}} 48^{\mathrm{s}}$ & $-34^{\circ} 29^{\prime} 48^{\prime \prime}$ & 307.7 & 28.2 & 5 & 820 & None \\
\hline 1FGL J1311.7-3429 & $13^{\mathrm{h}} 11^{\mathrm{m}} 48^{\mathrm{s}}$ & $-34^{\circ} 29^{\prime} 48^{\prime \prime}$ & 307.7 & 28.2 & 5 & 820 & None \\
\hline 1FGL J1312.6+0048 ${ }^{\dagger}$ & $13^{\mathrm{h}} 12^{\mathrm{m}} 42^{\mathrm{s}}$ & $-34^{\circ} 29^{\prime} 48^{\prime \prime}$ & 307.9 & 28.2 & 5 & 820 & None \\
\hline 1FGL J1416.2-1001 & $14^{\mathrm{h}} 15^{\mathrm{m}} 02^{\mathrm{s}}$ & $-10^{\circ} 01^{\prime} 24^{\prime \prime}$ & 334.4 & 47.7 & 45 & 820 & None \\
\hline SEED3Y-29 & $14^{\mathrm{h}} 18^{\mathrm{m}} 26^{\mathrm{s}}$ & $+35^{\circ} 42^{\prime} 21^{\prime \prime}$ & 63.2 & 69.6 & 40 & 820 & 3FGL J1418.5+3543 \\
\hline P7R42122 * & $14^{\mathrm{h}} 21^{\mathrm{m}} 02^{\mathrm{s}}$ & $-24^{\circ} 31^{\prime} 39^{\prime \prime}$ & 327.7 & 34.0 & 45 & 820 & 3FGL J1421.0-2431 \\
\hline P72Y2198 & $14^{\mathrm{h}} 22^{\mathrm{m}} 51^{\mathrm{s}}$ & $+58^{\circ} 02^{\prime} 55^{\prime \prime}$ & 101.8 & 55.2 & 45 & 820 & 3FGL J1422.8+5801 \\
\hline P72Y2219 & $14^{\mathrm{h}} 32^{\mathrm{m}} 59^{\mathrm{s}}$ & $+76^{\circ} 49^{\prime} 27^{\prime \prime}$ & 115.7 & 38.8 & 45 & 820 & None \\
\hline 2FGL J1511.8-0513 & $15^{\mathrm{h}} 11^{\mathrm{m}} 52^{\mathrm{s}}$ & $-05^{\circ} 13^{\prime} 23^{\prime \prime}$ & 354.6 & 43.1 & 45 & 820 & 3FGL J1511.8-0513 \\
\hline 2FGL J1513.5-2546 & $15^{\mathrm{h}} 13^{\mathrm{m}} 31^{\mathrm{s}}$ & $-25^{\circ} 46^{\prime} 01^{\prime \prime}$ & 338.9 & 27.0 & 35 & 350 & 3FGL J1513.4-2549 \\
\hline 2FGL J1513.5-2546 & $15^{\mathrm{h}} 13^{\mathrm{m}} 31^{\mathrm{s}}$ & $-25^{\circ} 46^{\prime} 01^{\prime \prime}$ & 338.9 & 27.0 & 35 & 350 & 3FGL J1513.4-2549 \\
\hline P7R42252* & $15^{\mathrm{h}} 13^{\mathrm{m}} 31^{\mathrm{s}}$ & $-25^{\circ} 49^{\prime} 52^{\prime \prime}$ & 338.9 & 27.0 & 35 & 820 & 3FGL J1513.4-2549 \\
\hline P7R42258 * & $15^{\mathrm{h}} 16^{\mathrm{m}} 46^{\mathrm{s}}$ & $+36^{\circ} 48^{\prime} 30^{\prime \prime}$ & 59.7 & 57.9 & 40 & 820 & 3FGL J1516.7+3648 \\
\hline 1FGL J1519.7+4216 & $15^{\mathrm{h}} 20^{\mathrm{m}} 41^{\mathrm{s}}$ & $+42^{\circ} 11^{\prime} 40^{\prime \prime}$ & 69.2 & 56.2 & 45 & 820 & None \\
\hline SEED3Y-284 & $15^{\mathrm{h}} 32^{\mathrm{m}} 40^{\mathrm{s}}$ & $-13^{\circ} 18^{\prime} 37^{\prime \prime}$ & 352.1 & 33.7 & 35 & 820 & 3FGL J1532.7-1319 \\
\hline 1FGL J1539.0-3328 & $15^{\mathrm{h}} 39^{\mathrm{m}} 10^{\mathrm{s}}$ & $-33^{\circ} 26^{\prime} 30^{\prime \prime}$ & 338.7 & 17.5 & 5 & 820 & None \\
\hline 2FGL J1539.2-3325 & $15^{\mathrm{h}} 39^{\mathrm{m}} 15^{\mathrm{s}}$ & $-33^{\circ} 25^{\prime} 42^{\prime \prime}$ & 338.7 & 17.5 & 5 & 820 & 3FGL J1539.2-3324 \\
\hline P7R42328 & $15^{\mathrm{h}} 44^{\mathrm{m}} 00^{\mathrm{s}}$ & $-25^{\circ} 55^{\prime} 46^{\prime \prime}$ & 344.7 & 22.6 & 45 & 820 & 3FGL J1544.1-2555 \\
\hline 1FGL J1544.5-1127 & $15^{\mathrm{h}} 44^{\mathrm{m}} 35^{\mathrm{s}}$ & $-11^{\circ} 25^{\prime} 33^{\prime \prime}$ & 356.2 & 33.0 & 5 & 820 & None \\
\hline P7R42354 * & $15^{\mathrm{h}} 53^{\mathrm{m}} 10^{\mathrm{s}}$ & $+54^{\circ} 37^{\prime} 27^{\prime \prime}$ & 85.6 & 47.2 & 45 & 820 & 3FGL J1553.1+5437 \\
\hline P72Y2431 & $15^{\mathrm{h}} 59^{\mathrm{m}} 57^{\mathrm{s}}$ & $+23^{\circ} 20^{\prime} 34^{\prime \prime}$ & 38.7 & 47.4 & 33 & 820 & 3FGL J1559.9+2319 \\
\hline P7R42383 * & $16^{\mathrm{h}} 01^{\mathrm{m}} 57^{\mathrm{s}}$ & $+23^{\circ} 06^{\prime} 38^{\prime \prime}$ & 38.5 & 46.9 & 45 & 820 & 3FGL J1601.9+2306 \\
\hline 2FGL J1602.4+2308 & $16^{\mathrm{h}} 02^{\mathrm{m}} 28^{\mathrm{s}}$ & $+23^{\circ} 08^{\prime} 27^{\prime \prime}$ & 38.6 & 46.8 & 50 & 820 & 3FGL J1601.9+2306 \\
\hline 2FGL J1602.4+2308 & $16^{\mathrm{h}} 02^{\mathrm{m}} 28^{\mathrm{s}}$ & $+23^{\circ} 08^{\prime} 27^{\prime \prime}$ & 38.6 & 46.8 & 5 & 820 & 3FGL J1601.9+2306 \\
\hline P7R42434 & $16^{\mathrm{h}} 22^{\mathrm{m}} 57^{\mathrm{s}}$ & $-03^{\circ} 12^{\prime} 13^{\prime \prime}$ & 10.8 & 30.7 & 45 & 820 & 3FGL J1622.9-0312 \\
\hline 2FGL J1624.2-2124 & $16^{\mathrm{h}} 24^{\mathrm{m}} 17^{\mathrm{s}}$ & $-21^{\circ} 24^{\prime} 39^{\prime \prime}$ & 355.1 & 19.3 & 35 & 350 & None \\
\hline P7R42443 * & $16^{\mathrm{h}} 25^{\mathrm{m}} 07^{\mathrm{s}}$ & $-03^{\circ} 12^{\prime} 13^{\prime \prime}$ & 11.1 & 30.3 & 45 & 820 & None \\
\hline
\end{tabular}


Table 5.6-Continued

\begin{tabular}{|c|c|c|c|c|c|c|c|}
\hline Source & $\begin{array}{c}\text { R.A. } \\
\text { J2000.0 }\end{array}$ & $\begin{array}{c}\text { Decl. } \\
\text { J2000.0 }\end{array}$ & $\begin{array}{c}l \\
\operatorname{deg}\end{array}$ & $\begin{array}{c}b \\
\operatorname{deg}\end{array}$ & $\begin{array}{l}\text { Integra- } \\
\text { tion Time } \\
\text { minutes }\end{array}$ & $\begin{array}{c}\text { Observing } \\
\text { Freq } \\
\mathrm{MHz}\end{array}$ & 3FGL name \\
\hline 2FGL J1625.2-0020 & $16^{\mathrm{h}} 25^{\mathrm{m}} 12^{\mathrm{s}}$ & $-01^{\circ} 41^{\prime} 11^{\prime \prime}$ & 12.6 & 31.1 & 45 & 820 & 3FGL J1625.1-0021 \\
\hline 1FGL J1625.3-0019 & $16^{\mathrm{h}} 25^{\mathrm{m}} 14^{\mathrm{s}}$ & $-34^{\circ} 29^{\prime} 48^{\prime \prime}$ & 345.2 & 10.3 & 45 & 820 & None \\
\hline 1FGL J1625.3-0019 & $16^{\mathrm{h}} 25^{\mathrm{m}} 14^{\mathrm{s}}$ & $-34^{\circ} 29^{\prime} 48^{\prime \prime}$ & 345.2 & 10.3 & 5 & 820 & None \\
\hline P7R42457 * & $16^{\mathrm{h}} 27^{\mathrm{m}} 52^{\mathrm{s}}$ & $+32^{\circ} 17^{\prime} 42^{\prime \prime}$ & 53.0 & 43.2 & 40 & 820 & 3FGL J1627.8+3217 \\
\hline 1FGL J1627.6+3218 & $16^{\mathrm{h}} 27^{\mathrm{m}} 54^{\mathrm{s}}$ & $+32^{\circ} 19^{\prime} 58^{\prime \prime}$ & 53.0 & 43.2 & 30 & 820 & None \\
\hline 1FGL J1627.6+3218 & $16^{\mathrm{h}} 27^{\mathrm{m}} 54^{\mathrm{s}}$ & $+32^{\circ} 19^{\prime} 58^{\prime \prime}$ & 53.0 & 43.2 & 5 & 820 & None \\
\hline 1FGL J1627.8-3204 & $16^{\mathrm{h}} 28^{\mathrm{m}} 10^{\mathrm{s}}$ & $-32^{\circ} 04^{\prime} 39^{\prime \prime}$ & 347.4 & 11.5 & 45 & 820 & None \\
\hline 2 FGL J1630.3+3732 ${ }^{\dagger}$ & $16^{\mathrm{h}} 30^{\mathrm{m}} 23^{\mathrm{s}}$ & $+37^{\circ} 32^{\prime} 55^{\prime \prime}$ & 60.2 & 43.3 & 11 & 820 & 3FGL J1630.2+3733 \\
\hline 2 FGL J1630.3+3732 ${ }^{\dagger}$ & $16^{\mathrm{h}} 30^{\mathrm{m}} 23^{\mathrm{s}}$ & $+37^{\circ} 32^{\prime} 55^{\prime \prime}$ & 60.2 & 43.3 & 12 & 820 & 3FGL J1630.2+3733 \\
\hline 2 FGL J1630.3+3732 $\dagger$ & $16^{\mathrm{h}} 30^{\mathrm{m}} 23^{\mathrm{s}}$ & $+37^{\circ} 32^{\prime} 55^{\prime \prime}$ & 60.2 & 43.3 & 5 & 820 & 3FGL J1630.2+3733 \\
\hline 2FGL J1632.6-2328c & $16^{\mathrm{h}} 32^{\mathrm{m}} 37^{\mathrm{s}}$ & $-23^{\circ} 28^{\prime} 21^{\prime \prime}$ & 354.8 & 16.4 & 17 & 350 & None \\
\hline P7R42530 * & $16^{\mathrm{h}} 49^{\mathrm{m}} 40^{\mathrm{s}}$ & $-30^{\circ} 07^{\prime} 45^{\prime \prime}$ & 352.0 & 9.3 & 45 & 820 & 3FGL J1649.6-3007 \\
\hline 2FGL J1653.6-0159 & $16^{\mathrm{h}} 53^{\mathrm{m}} 36^{\mathrm{s}}$ & $-01^{\circ} 59^{\prime} 47^{\prime \prime}$ & 16.6 & 24.9 & 49 & 820 & 3FGL J1653.6-0158 \\
\hline 2FGL J1653.6-0159 & $16^{\mathrm{h}} 53^{\mathrm{m}} 38^{\mathrm{s}}$ & $-01^{\circ} 58^{\prime} 36^{\prime \prime}$ & 16.6 & 24.9 & 10 & 820 & 3FGL J1653.6-0158 \\
\hline 1FGL J1653.6-0158 & $16^{\mathrm{h}} 53^{\mathrm{m}} 39^{\mathrm{s}}$ & $-01^{\circ} 58^{\prime} 21^{\prime \prime}$ & 16.6 & 24.9 & 45 & 820 & None \\
\hline P72Y2613 & $16^{\mathrm{h}} 56^{\mathrm{m}} 02^{\mathrm{s}}$ & $+20^{\circ} 46^{\prime} 17^{\prime \prime}$ & 40.6 & 34.3 & 53 & 820 & 3FGL J1656.0+2044 \\
\hline P72Y2627 & $16^{\mathrm{h}} 57^{\mathrm{m}} 58^{\mathrm{s}}$ & $+61^{\circ} 50^{\prime} 33^{\prime \prime}$ & 91.6 & 37.0 & 61 & 820 & None \\
\hline P7R42562 & $16^{\mathrm{h}} 59^{\mathrm{m}} 05^{\mathrm{s}}$ & $-01^{\circ} 42^{\prime} 06^{\prime \prime}$ & 17.6 & 23.9 & 45 & 820 & 3FGL J1659.0-0142 \\
\hline P7R42580G & $17^{\mathrm{h}} 03^{\mathrm{m}} 40^{\mathrm{s}}$ & $-28^{\circ} 51^{\prime} 32^{\prime \prime}$ & 354.9 & 7.6 & 45 & 820 & 3FGL J1703.6-2850 \\
\hline P7R42603 * & $17^{\mathrm{h}} 11^{\mathrm{m}} 46^{\mathrm{s}}$ & $-19^{\circ} 23^{\prime} 21^{\prime \prime}$ & 3.8 & 11.7 & 57 & 350 & None \\
\hline P7R42606G & $17^{\mathrm{h}} 13^{\mathrm{m}} 52^{\mathrm{s}}$ & $-29^{\circ} 27^{\prime} 14^{\prime \prime}$ & 355.7 & 5.5 & 45 & 820 & None \\
\hline P7R42606G & $17^{\mathrm{h}} 13^{\mathrm{m}} 52^{\mathrm{s}}$ & $-29^{\circ} 27^{\prime} 14^{\prime \prime}$ & 355.7 & 5.5 & 45 & 820 & None \\
\hline P72Y2678 & $17^{\mathrm{h}} 14^{\mathrm{m}} 00^{\mathrm{s}}$ & $-06^{\circ} 16^{\prime} 22^{\prime \prime}$ & 15.5 & 18.4 & 22 & 350 & None \\
\hline 2FGL J1716.6-0526c & $17^{\mathrm{h}} 16^{\mathrm{m}} 40^{\mathrm{s}}$ & $-05^{\circ} 26^{\prime} 28^{\prime \prime}$ & 16.6 & 18.2 & 5 & 820 & None \\
\hline P7R42631 & $17^{\mathrm{h}} 20^{\mathrm{m}} 45^{\mathrm{s}}$ & $+07^{\circ} 11^{\prime} 21^{\prime \prime}$ & 29.0 & 23.4 & 28 & 820 & 3 FGL J1720.7+0711 \\
\hline 2FGL J1722.5-0420 & $17^{\mathrm{h}} 22^{\mathrm{m}} 34^{\mathrm{s}}$ & $-04^{\circ} 20^{\prime} 40^{\prime \prime}$ & 18.4 & 17.5 & 39 & 820 & 3FGL J1722.7-0415 \\
\hline 2FGL J1727.8-2308 & $17^{\mathrm{h}} 27^{\mathrm{m}} 50^{\mathrm{s}}$ & $-23^{\circ} 08^{\prime} 42^{\prime \prime}$ & 2.8 & 6.5 & 42 & 820 & None \\
\hline 2FGL J1727.8-2308 & $17^{\mathrm{h}} 27^{\mathrm{m}} 50^{\mathrm{s}}$ & $-23^{\circ} 08^{\prime} 42^{\prime \prime}$ & 2.8 & 6.5 & 5 & 820 & None \\
\hline P7R42654G & $17^{\mathrm{h}} 28^{\mathrm{m}} 13^{\mathrm{s}}$ & $-16^{\circ} 10^{\prime} 08^{\prime \prime}$ & 8.7 & 10.2 & 45 & 820 & None \\
\hline 1FGL J1730.7-0352 & $17^{\mathrm{h}} 30^{\mathrm{m}} 36^{\mathrm{s}}$ & $-03^{\circ} 53^{\prime} 17^{\prime \prime}$ & 19.9 & 16.0 & 5 & 820 & None \\
\hline 2FGL J1730.6-2409 & $17^{\mathrm{h}} 30^{\mathrm{m}} 37^{\mathrm{s}}$ & $-24^{\circ} 09^{\prime} 39^{\prime \prime}$ & 2.3 & 5.4 & 50 & 820 & 3FGL J1729.7-2408 \\
\hline 2FGL J1730.6-0353 & $17^{\mathrm{h}} 30^{\mathrm{m}} 38^{\mathrm{s}}$ & $-03^{\circ} 53^{\prime} 42^{\prime \prime}$ & 19.9 & 16.0 & 45 & 820 & 3FGL J1730.6-0357 \\
\hline 2FGL J1730.6-0353 & $17^{\mathrm{h}} 30^{\mathrm{m}} 38^{\mathrm{s}}$ & $-03^{\circ} 53^{\prime} 42^{\prime \prime}$ & 19.9 & 16.0 & 50 & 820 & 3FGL J1730.6-0357 \\
\hline 2FGL J1730.6-0353 & $17^{\mathrm{h}} 30^{\mathrm{m}} 38^{\mathrm{s}}$ & $-03^{\circ} 53^{\prime} 42^{\prime \prime}$ & 19.9 & 16.0 & 50 & 820 & 3FGL J1730.6-0357 \\
\hline 2FGL J1730.6-0353 & $17^{\mathrm{h}} 30^{\mathrm{m}} 38^{\mathrm{s}}$ & $-03^{\circ} 53^{\prime} 42^{\prime \prime}$ & 19.9 & 16.0 & 5 & 820 & 3FGL J1730.6-0357 \\
\hline P7R42709A * & $17^{\mathrm{h}} 43^{\mathrm{m}} 58^{\mathrm{s}}$ & $-13^{\circ} 10^{\prime} 42^{\prime \prime}$ & 13.3 & 8.5 & 40 & 820 & 3FGL J1743.9-1310 \\
\hline 1FGL J1746.7-3233 & $17^{\mathrm{h}} 46^{\mathrm{m}} 40^{\mathrm{s}}$ & $-32^{\circ} 36^{\prime} 17^{\prime \prime}$ & 357.0 & -2.1 & 45 & 820 & None \\
\hline 1FGL J1746.7-3233 & $17^{\mathrm{h}} 46^{\mathrm{m}} 40^{\mathrm{s}}$ & $-32^{\circ} 36^{\prime} 17^{\prime \prime}$ & 357.0 & -2.1 & 45 & 820 & None \\
\hline 1FGL J1746.7-3233 & $17^{\mathrm{h}} 46^{\mathrm{m}} 40^{\mathrm{s}}$ & $-32^{\circ} 36^{\prime} 17^{\prime \prime}$ & 357.0 & -2.1 & 5 & 820 & None \\
\hline 1FGL J1746.7-3233 & $17^{\mathrm{h}} 46^{\mathrm{m}} 40^{\mathrm{s}}$ & $-32^{\circ} 36^{\prime} 17^{\prime \prime}$ & 357.0 & -2.1 & 5 & 820 & None \\
\hline P72Y2820 & $17^{\mathrm{h}} 49^{\mathrm{m}} 48^{\mathrm{s}}$ & $-03^{\circ} 05^{\prime} 26^{\prime \prime}$ & 23.0 & 12.2 & 27 & 820 & 3FGL J1749.7-0305 \\
\hline
\end{tabular}


Table 5.6-Continued

\begin{tabular}{|c|c|c|c|c|c|c|c|}
\hline Source & $\begin{array}{c}\text { R.A. } \\
\text { J2000.0 }\end{array}$ & $\begin{array}{c}\text { Decl. } \\
\text { J2000.0 }\end{array}$ & $\begin{array}{c}l \\
\operatorname{deg}\end{array}$ & $\begin{array}{c}b \\
\operatorname{deg}\end{array}$ & $\begin{array}{l}\text { Integra- } \\
\text { tion Time } \\
\text { minutes }\end{array}$ & $\begin{array}{c}\text { Observing } \\
\text { Freq } \\
\mathrm{MHz}\end{array}$ & 3FGL name \\
\hline 1FGL J1749.5-0301 & $17^{\mathrm{h}} 49^{\mathrm{m}} 51^{\mathrm{s}}$ & $-03^{\circ} 04^{\prime} 20^{\prime \prime}$ & 23.0 & 12.2 & 5 & 820 & None \\
\hline SEED_09-94 & $17^{\mathrm{h}} 57^{\mathrm{m}} 11^{\mathrm{s}}$ & $+70^{\circ} 31^{\prime} 48^{\prime \prime}$ & 101.0 & 30.0 & 45 & 820 & 3FGL J1756.9+7032 \\
\hline 1FGL J1806.2+0609 & $18^{\mathrm{h}} 05^{\mathrm{m}} 50^{\mathrm{s}}$ & $+06^{\circ} 12^{\prime} 27^{\prime \prime}$ & 33.3 & 13.0 & 45 & 820 & None \\
\hline 1FGL J1806.2+0609 & $18^{\mathrm{h}} 05^{\mathrm{m}} 50^{\mathrm{s}}$ & $+06^{\circ} 12^{\prime} 27^{\prime \prime}$ & 33.3 & 13.0 & 5 & 820 & None \\
\hline 2FGL J1805.8+0612 & $18^{\mathrm{h}} 05^{\mathrm{m}} 51^{\mathrm{s}}$ & $+06^{\circ} 12^{\prime} 48^{\prime \prime}$ & 33.3 & 13.0 & 45 & 820 & 3FGL J1805.9+0614 \\
\hline SEED_09-122 & $18^{\mathrm{h}} 08^{\mathrm{m}} 00^{\mathrm{s}}$ & $+34^{\circ} 54^{\prime} 44^{\prime \prime}$ & 61.5 & 23.5 & 50 & 820 & 3FGL J1809.0+3517 \\
\hline 2FGL J1808.3-3356 & $18^{\mathrm{h}} 08^{\mathrm{m}} 22^{\mathrm{s}}$ & $-33^{\circ} 56^{\prime} 04^{\prime \prime}$ & 358.1 & -6.7 & 50 & 820 & 3FGL J1808.3-3357 \\
\hline P7R42794* & $18^{\mathrm{h}} 08^{\mathrm{m}} 23^{\mathrm{s}}$ & $-33^{\circ} 57^{\prime} 46^{\prime \prime}$ & 358.1 & -6.7 & 63 & 820 & 3FGL J1808.3-3357 \\
\hline P7R42794* & $18^{\mathrm{h}} 08^{\mathrm{m}} 23^{\mathrm{s}}$ & $-33^{\circ} 57^{\prime} 47^{\prime \prime}$ & 358.1 & -6.7 & 45 & 820 & 3FGL J1808.3-3357 \\
\hline 2FGL J1729.5-0854 & $18^{\mathrm{h}} 10^{\mathrm{m}} 18^{\mathrm{s}}$ & $-11^{\circ} 03^{\prime} 57^{\prime \prime}$ & 18.4 & 3.9 & 35 & 350 & 3FGL J1729.9-0859 \\
\hline 2FGL J1816.5+4511 & $18^{\mathrm{h}} 16^{\mathrm{m}} 35^{\mathrm{s}}$ & $+45^{\circ} 11^{\prime} 57^{\prime \prime}$ & 72.9 & 24.7 & 21 & 820 & 3FGL J1816.5+4512 \\
\hline $24 \mathrm{M} 2244$ & $18^{\mathrm{h}} 19^{\mathrm{m}} 23^{\mathrm{s}}$ & $-15^{\circ} 31^{\prime} 12^{\prime \prime}$ & 15.5 & -0.1 & 5 & 820 & 3FGL J1818.7-1528 \\
\hline P7R42830B * & $18^{\mathrm{h}} 20^{\mathrm{m}} 24^{\mathrm{s}}$ & $-32^{\circ} 17^{\prime} 09^{\prime \prime}$ & 0.7 & -8.2 & 39 & 350 & 3FGL J1820.4-3217 \\
\hline 2FGL J1820.6-3219 & $18^{\mathrm{h}} 20^{\mathrm{m}} 38^{\mathrm{s}}$ & $-32^{\circ} 19^{\prime} 23^{\prime \prime}$ & 0.7 & -8.2 & 50 & 820 & 3FGL J1820.4-3217 \\
\hline SEED3Y-344 & $18^{\mathrm{h}} 22^{\mathrm{m}} 26^{\mathrm{s}}$ & $+66^{\circ} 34^{\prime} 47^{\prime \prime}$ & 96.5 & 27.6 & 40 & 820 & None \\
\hline P7R42854* & $18^{\mathrm{h}} 27^{\mathrm{m}} 20^{\mathrm{s}}$ & $-14^{\circ} 46^{\prime} 01^{\prime \prime}$ & 17.1 & -1.5 & 35 & 820 & 3FGL J1826.2-1450 \\
\hline P7R42856 & $18^{\mathrm{h}} 27^{\mathrm{m}} 42^{\mathrm{s}}$ & $+11^{\circ} 41^{\prime} 50^{\prime \prime}$ & 40.8 & 10.5 & 45 & 820 & 3FGL J1827.7+1141 \\
\hline 2FGL J1829.8-0204c & $18^{\mathrm{h}} 29^{\mathrm{m}} 48^{\mathrm{s}}$ & $-02^{\circ} 04^{\prime} 35^{\prime \prime}$ & 28.6 & 3.9 & 34 & 820 & 3FGL J1829.9-0203c \\
\hline 2FGL J1829.8-0204c & $18^{\mathrm{h}} 29^{\mathrm{m}} 48^{\mathrm{s}}$ & $-02^{\circ} 04^{\prime} 35^{\prime \prime}$ & 28.6 & 3.9 & 5 & 820 & 3FGL J1829.9-0203c \\
\hline P7R42871 * & $18^{\mathrm{h}} 30^{\mathrm{m}} 50^{\mathrm{s}}$ & $-31^{\circ} 36^{\prime} 09^{\prime \prime}$ & 2.4 & -9.8 & 50 & 820 & 3FGL J1830.8-3136 \\
\hline 2FGL J1832.0-0200 & $18^{\mathrm{h}} 32^{\mathrm{m}} 00^{\mathrm{s}}$ & $-02^{\circ} 00^{\prime} 59^{\prime \prime}$ & 28.9 & 3.4 & 44 & 820 & 3FGL J1831.7-0157c \\
\hline 2FGL J1832.0-0200 & $18^{\mathrm{h}} 32^{\mathrm{m}} 00^{\mathrm{s}}$ & $-02^{\circ} 00^{\prime} 59^{\prime \prime}$ & 28.9 & 3.4 & 5 & 820 & 3FGL J1831.7-0157c \\
\hline P7R42896 * & $18^{\mathrm{h}} 39^{\mathrm{m}} 23^{\mathrm{s}}$ & $-05^{\circ} 52^{\prime} 53^{\prime \prime}$ & 26.3 & -0.0 & 33 & 820 & 3FGL J1838.9-0537 \\
\hline $24 \mathrm{M} 2314$ & $18^{\mathrm{h}} 42^{\mathrm{m}} 07^{\mathrm{s}}$ & $+27^{\circ} 40^{\prime} 13^{\prime \prime}$ & 57.1 & 14.1 & 45 & 820 & 3FGL J1842.2+2742 \\
\hline $24 \mathrm{M} 2314$ & $18^{\mathrm{h}} 42^{\mathrm{m}} 07^{\mathrm{s}}$ & $+27^{\circ} 40^{\prime} 13^{\prime \prime}$ & 57.1 & 14.1 & 5 & 820 & 3FGL J1842.2+2742 \\
\hline P7R42910 & $18^{\mathrm{h}} 42^{\mathrm{m}} 15^{\mathrm{s}}$ & $+27^{\circ} 42^{\prime} 08^{\prime \prime}$ & 57.1 & 14.1 & 50 & 820 & 3FGL J1842.2+2742 \\
\hline 2FGL J1842.3+2740 & $18^{\mathrm{h}} 42^{\mathrm{m}} 19^{\mathrm{s}}$ & $+27^{\circ} 40^{\prime} 15^{\prime \prime}$ & 57.1 & 14.1 & 35 & 350 & 3FGL J1842.2+2742 \\
\hline P7R42921 & $18^{\mathrm{h}} 45^{\mathrm{m}} 32^{\mathrm{s}}$ & $-25^{\circ} 24^{\prime} 32^{\prime \prime}$ & 9.4 & -10.1 & 40 & 820 & 3FGL J1845.5-2524 \\
\hline P72Y3022 & $18^{\mathrm{h}} 48^{\mathrm{m}} 24^{\mathrm{s}}$ & $+32^{\circ} 31^{\prime} 43^{\prime \prime}$ & 62.3 & 14.8 & 56 & 820 & 3FGL J1848.4+3216 \\
\hline P7R42929 * & $18^{\mathrm{h}} 48^{\mathrm{m}} 38^{\mathrm{s}}$ & $+32^{\circ} 33^{\prime} 26^{\prime \prime}$ & 62.3 & 14.7 & 45 & 820 & 3FGL J1848.4+3216 \\
\hline P7R42948GP $\dagger$ & $18^{\mathrm{h}} 55^{\mathrm{m}} 57^{\mathrm{s}}$ & $-14^{\circ} 38^{\prime} 55^{\prime \prime}$ & 20.3 & -7.6 & 45 & 820 & None \\
\hline 1FGL J1858.1-2218 † & $18^{\mathrm{h}} 58^{\mathrm{m}} 28^{\mathrm{s}}$ & $-22^{\circ} 18^{\prime} 55^{\prime \prime}$ & 13.6 & -11.4 & 46 & 820 & None \\
\hline 1FGL J1858.1-2218 ${ }^{\dagger}$ & $18^{\mathrm{h}} 58^{\mathrm{m}} 28^{\mathrm{s}}$ & $-22^{\circ} 18^{\prime} 55^{\prime \prime}$ & 13.6 & -11.4 & 5 & 820 & None \\
\hline 1FGL J1858.1-2218 † & $18^{\mathrm{h}} 58^{\mathrm{m}} 28^{\mathrm{s}}$ & $-22^{\circ} 18^{\prime} 55^{\prime \prime}$ & 13.6 & -11.4 & 5 & 820 & None \\
\hline P7R42980 & $19^{\mathrm{h}} 04^{\mathrm{m}} 47^{\mathrm{s}}$ & $-07^{\circ} 08^{\prime} 17^{\prime \prime}$ & 28.1 & -6.2 & 35 & 820 & 3FGL J1904.7-0708 \\
\hline 2FGL J1904.9-3720c & $19^{\mathrm{h}} 04^{\mathrm{m}} 55^{\mathrm{s}}$ & $-37^{\circ} 20^{\prime} 00^{\prime \prime}$ & 359.8 & -18.5 & 25 & 820 & None \\
\hline 2FGL J1904.9-3720c & $19^{\mathrm{h}} 04^{\mathrm{m}} 55^{\mathrm{s}}$ & $-37^{\circ} 20^{\prime} 00^{\prime \prime}$ & 359.8 & -18.5 & 5 & 820 & None \\
\hline P72Y3081 & $19^{\mathrm{h}} 06^{\mathrm{m}} 38^{\mathrm{s}}$ & $+07^{\circ} 19^{\prime} 18^{\prime \prime}$ & 41.2 & -0.0 & 45 & 820 & 3FGL J1906.6+0720 \\
\hline 1FGL J1908.5-0138 & $19^{\mathrm{h}} 08^{\mathrm{m}} 51^{\mathrm{s}}$ & $-01^{\circ} 34^{\prime} 43^{\prime \prime}$ & 33.5 & -4.6 & 5 & 820 & None \\
\hline P7R42990A * & $19^{\mathrm{h}} 09^{\mathrm{m}} 32^{\mathrm{s}}$ & $+21^{\circ} 02^{\prime} 54^{\prime \prime}$ & 53.7 & 5.6 & 45 & 350 & None \\
\hline P7R43023A *† & $19^{\mathrm{h}} 21^{\mathrm{m}} 13^{\mathrm{s}}$ & $+01^{\circ} 36^{\prime} 27^{\prime \prime}$ & 37.8 & -5.9 & 45 & 820 & 3FGL J1921.2+0136 \\
\hline
\end{tabular}


Table 5.6-Continued

\begin{tabular}{|c|c|c|c|c|c|c|c|}
\hline Source & $\begin{array}{c}\text { R.A. } \\
\text { J2000.0 }\end{array}$ & $\begin{array}{c}\text { Decl. } \\
\text { J2000.0 }\end{array}$ & $\begin{array}{c}l \\
\operatorname{deg}\end{array}$ & $\begin{array}{c}b \\
\operatorname{deg}\end{array}$ & $\begin{array}{l}\text { Integra- } \\
\text { tion Time } \\
\text { minutes }\end{array}$ & $\begin{array}{c}\text { Observing } \\
\text { Freq } \\
\mathrm{MHz}\end{array}$ & 3FGL name \\
\hline P7R43038 * & $19^{\mathrm{h}} 25^{\mathrm{m}} 29^{\mathrm{s}}$ & $+17^{\circ} 27^{\prime} 47^{\prime \prime}$ & 52.3 & 0.6 & 35 & 820 & 3FGL J1925.4+1727 \\
\hline P7R43071 & $19^{\mathrm{h}} 44^{\mathrm{m}} 08^{\mathrm{s}}$ & $+39^{\circ} 19^{\prime} 51^{\prime \prime}$ & 73.5 & 7.6 & 25 & 820 & 3FGL J1944.1+3919 \\
\hline P7R43082 & $19^{\mathrm{h}} 49^{\mathrm{m}} 01^{\mathrm{s}}$ & $+13^{\circ} 12^{\prime} 36^{\prime \prime}$ & 51.3 & -6.4 & 45 & 820 & 3FGL J1949.0+1312 \\
\hline SEED3Y-762 & $19^{\mathrm{h}} 49^{\mathrm{m}} 09^{\mathrm{s}}$ & $+13^{\circ} 10^{\prime} 51^{\prime \prime}$ & 51.3 & -6.4 & 45 & 820 & 3FGL J1949.0+1312 \\
\hline P72Y3203 & $19^{\mathrm{h}} 55^{\mathrm{m}} 50^{\mathrm{s}}$ & $+02^{\circ} 12^{\prime} 52^{\prime \prime}$ & 42.4 & -13.2 & 45 & 820 & 3FGL J1955.9+0212 \\
\hline P7R43125 * & $20^{\mathrm{h}} 00^{\mathrm{m}} 06^{\mathrm{s}}$ & $+42^{\circ} 12^{\prime} 33^{\prime \prime}$ & 77.5 & 6.4 & 45 & 820 & 3FGL J2000.1+4212 \\
\hline P7R43137 & $20^{\mathrm{h}} 04^{\mathrm{m}} 52^{\mathrm{s}}$ & $+70^{\circ} 03^{\prime} 33^{\prime \prime}$ & 102.9 & 19.5 & 45 & 820 & 3FGL J2004.8+7003 \\
\hline P7R43143 * & $20^{\mathrm{h}} 06^{\mathrm{m}} 38^{\mathrm{s}}$ & $+01^{\circ} 50^{\prime} 45^{\prime \prime}$ & 43.4 & -15.8 & 29 & 820 & 3FGL J2006.6+0150 \\
\hline P7R43172 & $20^{\mathrm{h}} 16^{\mathrm{m}} 24^{\mathrm{s}}$ & $-09^{\circ} 05^{\prime} 00^{\prime \prime}$ & 34.3 & -23.0 & 45 & 820 & 3FGL J2016.4-0905 \\
\hline P7R43174 * & $20^{\mathrm{h}} 17^{\mathrm{m}} 41^{\mathrm{s}}$ & $-16^{\circ} 16^{\prime} 06^{\prime \prime}$ & 27.3 & -26.2 & 50 & 820 & 3FGL J2017.6-1616 \\
\hline $24 \mathrm{M} 2527.1$ & $20^{\mathrm{h}} 17^{\mathrm{m}} 57^{\mathrm{s}}$ & $+36^{\circ} 27^{\prime} 37^{\prime \prime}$ & 74.5 & 0.4 & 5 & 820 & 3FGL J2017.9+3627 \\
\hline P7R43179 & $20^{\mathrm{h}} 21^{\mathrm{m}} 56^{\mathrm{s}}$ & $+06^{\circ} 30^{\prime} 09^{\prime \prime}$ & 49.6 & -16.8 & 50 & 350 & 3FGL J2021.9+0630 \\
\hline P7R43195 & $20^{\mathrm{h}} 26^{\mathrm{m}} 20^{\mathrm{s}}$ & $+14^{\circ} 30^{\prime} 51^{\prime \prime}$ & 57.3 & -13.4 & 50 & 350 & 3FGL J2026.3+1430 \\
\hline 1FGL J2027.6+3335 & $20^{\mathrm{h}} 28^{\mathrm{m}} 18^{\mathrm{s}}$ & $+33^{\circ} 32^{\prime} 52^{\prime \prime}$ & 73.4 & -3.0 & 5 & 820 & None \\
\hline 1FGL J2027.6+3335 & $20^{\mathrm{h}} 28^{\mathrm{m}} 19^{\mathrm{s}}$ & $+33^{\circ} 32^{\prime} 52^{\prime \prime}$ & 73.4 & -3.0 & 45 & 820 & None \\
\hline P7R43197G * & $20^{\mathrm{h}} 28^{\mathrm{m}} 32^{\mathrm{s}}$ & $+40^{\circ} 40^{\prime} 37^{\prime \prime}$ & 79.2 & 1.1 & 34 & 820 & 3 FGL J2028.5+4040c \\
\hline 1FGL J2030.9+4411 & $20^{\mathrm{h}} 30^{\mathrm{m}} 54^{\mathrm{s}}$ & $+44^{\circ} 16^{\prime} 08^{\prime \prime}$ & 82.4 & 2.9 & 5 & 820 & None \\
\hline P72Y3322 & $20^{\mathrm{h}} 33^{\mathrm{m}} 54^{\mathrm{s}}$ & $+63^{\circ} 10^{\prime} 35^{\prime \prime}$ & 98.2 & 13.5 & 40 & 820 & 3FGL J2033.6+6309 \\
\hline 1FGL J2034.7+3639 & $20^{\mathrm{h}} 34^{\mathrm{m}} 56^{\mathrm{s}}$ & $+36^{\circ} 32^{\prime} 40^{\prime \prime}$ & 76.6 & -2.3 & 45 & 820 & None \\
\hline 1FGL J2034.7+3639 & $20^{\mathrm{h}} 34^{\mathrm{m}} 56^{\mathrm{s}}$ & $+36^{\circ} 32^{\prime} 40^{\prime \prime}$ & 76.6 & -2.3 & 5 & 820 & None \\
\hline P7R43222 & $20^{\mathrm{h}} 36^{\mathrm{m}} 25^{\mathrm{s}}$ & $+65^{\circ} 51^{\prime} 25^{\prime \prime}$ & 100.6 & 14.8 & 45 & 820 & 3FGL J2036.4+6551 \\
\hline 2FGL J2041.2+4735 & $20^{\mathrm{h}} 41^{\mathrm{m}} 17^{\mathrm{s}}$ & $+47^{\circ} 35^{\prime} 39^{\prime \prime}$ & 86.1 & 3.4 & 5 & 820 & 3FGL J2041.1+4736 \\
\hline $24 \mathrm{M} 2586^{\dagger}$ & $20^{\mathrm{h}} 42^{\mathrm{m}} 19^{\mathrm{s}}$ & $+02^{\circ} 49^{\prime} 46^{\prime \prime}$ & 49.1 & -23.0 & 5 & 820 & 3FGL J2042.1+0247 \\
\hline 2FGL J2046.0+4954 & $20^{\mathrm{h}} 46^{\mathrm{m}} 05^{\mathrm{s}}$ & $+49^{\circ} 54^{\prime} 45^{\prime \prime}$ & 88.4 & 4.2 & 35 & 350 & 3 FGL J2045.2+5026e \\
\hline 1FGL J2047.6+1055 & $20^{\mathrm{h}} 47^{\mathrm{m}} 04^{\mathrm{s}}$ & $+10^{\circ} 55^{\prime} 26^{\prime \prime}$ & 57.1 & -19.6 & 5 & 820 & None \\
\hline $24 \mathrm{M} 2616$ & $20^{\mathrm{h}} 52^{\mathrm{m}} 52^{\mathrm{s}}$ & $+31^{\circ} 44^{\prime} 24^{\prime \prime}$ & 75.1 & -8.2 & 5 & 820 & None \\
\hline 1FGL J2055.2+3144 & $20^{\mathrm{h}} 55^{\mathrm{m}} 30^{\mathrm{s}}$ & $+31^{\circ} 38^{\prime} 02^{\prime \prime}$ & 75.4 & -8.7 & 5 & 820 & None \\
\hline 1FGL J2055.2+3144 & $20^{\mathrm{h}} 55^{\mathrm{m}} 30^{\mathrm{s}}$ & $+31^{\circ} 38^{\prime} 03^{\prime \prime}$ & 75.4 & -8.7 & 45 & 820 & None \\
\hline 1FGL J2055.2+3144 & $20^{\mathrm{h}} 55^{\mathrm{m}} 30^{\mathrm{s}}$ & $+31^{\circ} 38^{\prime} 03^{\prime \prime}$ & 75.4 & -8.7 & 5 & 820 & None \\
\hline SEED_09-262 & $20^{\mathrm{h}} 56^{\mathrm{m}} 19^{\mathrm{s}}$ & $+31^{\circ} 41^{\prime} 44^{\prime \prime}$ & 75.5 & -8.8 & 50 & 820 & None \\
\hline 1FGL J2057.4+3057 & $20^{\mathrm{h}} 56^{\mathrm{m}} 49^{\mathrm{s}}$ & $+30^{\circ} 52^{\prime} 43^{\prime \prime}$ & 74.9 & -9.4 & 45 & 820 & None \\
\hline 1FGL J2057.4+3057 & $20^{\mathrm{h}} 56^{\mathrm{m}} 49^{\mathrm{s}}$ & $+30^{\circ} 52^{\prime} 43^{\prime \prime}$ & 74.9 & -9.4 & 5 & 820 & None \\
\hline P7R43290 * & $21^{\mathrm{h}} 03^{\mathrm{m}} 46^{\mathrm{s}}$ & $-11^{\circ} 13^{\prime} 44^{\prime \prime}$ & 37.9 & -34.4 & 50 & 820 & 3FGL J2103.7-1113 \\
\hline 2FGL J2107.9+5207c & $21^{\mathrm{h}} 07^{\mathrm{m}} 56^{\mathrm{s}}$ & $+52^{\circ} 07^{\prime} 08^{\prime \prime}$ & 92.3 & 3.1 & 50 & 820 & 3FGL J2108.1+5202 \\
\hline P7R43304 & $21^{\mathrm{h}} 08^{\mathrm{m}} 05^{\mathrm{s}}$ & $+52^{\circ} 01^{\prime} 48^{\prime \prime}$ & 92.3 & 3.0 & 35 & 820 & 3FGL J2108.1+5202 \\
\hline 2FGL J2112.5-3042 & $21^{\mathrm{h}} 12^{\mathrm{m}} 35^{\mathrm{s}}$ & $-30^{\circ} 42^{\prime} 37^{\prime \prime}$ & 14.9 & -42.4 & 5 & 820 & 3FGL J2112.5-3044 \\
\hline 1FGL J2112.5-3044 & $21^{\mathrm{h}} 12^{\mathrm{m}} 36^{\mathrm{s}}$ & $-30^{\circ} 42^{\prime} 33^{\prime \prime}$ & 14.9 & -42.4 & 5 & 820 & None \\
\hline P7R43324 * & $21^{\mathrm{h}} 14^{\mathrm{m}} 54^{\mathrm{s}}$ & $+54^{\circ} 48^{\prime} 56^{\prime \prime}$ & 95.0 & 4.1 & 50 & 820 & 3FGL J2114.9+5448 \\
\hline 1FGL J2116.8+3729 & $21^{\mathrm{h}} 17^{\mathrm{m}} 19^{\mathrm{s}}$ & $+37^{\circ} 27^{\prime} 21^{\prime \prime}$ & 82.8 & -8.2 & 5 & 820 & None \\
\hline 2FGL J2117.5+3730 & $21^{\mathrm{h}} 17^{\mathrm{m}} 30^{\mathrm{s}}$ & $+37^{\circ} 30^{\prime} 43^{\prime \prime}$ & 82.8 & -8.2 & 29 & 350 & 3FGL J2117.6+3725 \\
\hline 2FGL J2117.5+3730 & $21^{\mathrm{h}} 17^{\mathrm{m}} 32^{\mathrm{s}}$ & $+37^{\circ} 30^{\prime} 51^{\prime \prime}$ & 82.8 & -8.2 & 5 & 820 & 3FGL J2117.6+3725 \\
\hline
\end{tabular}


Table 5.6-Continued

\begin{tabular}{|c|c|c|c|c|c|c|c|}
\hline Source & $\begin{array}{c}\text { R.A. } \\
\text { J2000.0 }\end{array}$ & $\begin{array}{c}\text { Decl. } \\
\text { J2000.0 }\end{array}$ & $\begin{array}{c}l \\
\operatorname{deg}\end{array}$ & $\begin{array}{c}b \\
\operatorname{deg}\end{array}$ & $\begin{array}{l}\text { Integra- } \\
\text { tion Time } \\
\text { minutes }\end{array}$ & $\begin{array}{c}\text { Observing } \\
\text { Freq } \\
\mathrm{MHz}\end{array}$ & 3FGL name \\
\hline 2FGL J2117.5+3730 & $21^{\mathrm{h}} 17^{\mathrm{m}} 32^{\mathrm{s}}$ & $+37^{\circ} 30^{\prime} 52^{\prime \prime}$ & 82.8 & -8.2 & 35 & 350 & 3FGL J2117.6+3725 \\
\hline 2FGL J2128.7+5824 & $21^{\mathrm{h}} 28^{\mathrm{m}} 42^{\mathrm{s}}$ & $+58^{\circ} 24^{\prime} 21^{\prime \prime}$ & 98.9 & 5.3 & 30 & 350 & None \\
\hline SEED3Y-1251 & $21^{\mathrm{h}} 31^{\mathrm{m}} 07^{\mathrm{s}}$ & $-27^{\circ} 41^{\prime} 45^{\prime \prime}$ & 20.1 & -45.9 & 50 & 820 & $3 F G L$ J2130.8-2745 \\
\hline 1FGL J2139.9+4715 & $21^{\mathrm{h}} 39^{\mathrm{m}} 53^{\mathrm{s}}$ & $+47^{\circ} 13^{\prime} 29^{\prime \prime}$ & 92.6 & -4.0 & 45 & 820 & None \\
\hline 1FGL J2139.9+4715 & $21^{\mathrm{h}} 39^{\mathrm{m}} 53^{\mathrm{s}}$ & $+47^{\circ} 13^{\prime} 29^{\prime \prime}$ & 92.6 & -4.0 & 5 & 820 & None \\
\hline P72Y3547 & $21^{\mathrm{h}} 52^{\mathrm{m}} 33^{\mathrm{s}}$ & $+41^{\circ} 53^{\prime} 11^{\prime \prime}$ & 90.8 & -9.6 & 50 & 820 & 3FGL J2151.6+4154 \\
\hline P72Y3580 & $22^{\mathrm{h}} 12^{\mathrm{m}} 33^{\mathrm{s}}$ & $+28^{\circ} 00^{\prime} 06^{\prime \prime}$ & 85.1 & -23.0 & 50 & 820 & 3 FGL J2212.6+2801 \\
\hline P7R43453 * & $22^{\mathrm{h}} 12^{\mathrm{m}} 35^{\mathrm{s}}$ & $+07^{\circ} 03^{\prime} 35^{\prime \prime}$ & 68.7 & -38.6 & 45 & 350 & $3 F G L$ J2212.5+0703 \\
\hline 2FGL J2212.6+0702 & $22^{\mathrm{h}} 12^{\mathrm{m}} 37^{\mathrm{s}}$ & $+07^{\circ} 02^{\prime} 53^{\prime \prime}$ & 68.7 & -38.6 & 50 & 820 & 3 FGL J2212.5+0703 \\
\hline 2FGL J2221.0+6307 & $22^{\mathrm{h}} 21^{\mathrm{m}} 02^{\mathrm{s}}$ & $+63^{\circ} 08^{\prime} 03^{\prime \prime}$ & 106.9 & 5.1 & 27 & 350 & 3 FGL J2221.7+6318 \\
\hline 2FGL J2221.0+6307 & $22^{\mathrm{h}} 21^{\mathrm{m}} 03^{\mathrm{s}}$ & $+63^{\circ} 07^{\prime} 54^{\prime \prime}$ & 106.9 & 5.1 & 50 & 820 & 3 FGL J2221.7+6318 \\
\hline 2FGL J2227.8+0051 & $22^{\mathrm{h}} 27^{\mathrm{m}} 51^{\mathrm{s}}$ & $+63^{\circ} 07^{\prime} 54^{\prime \prime}$ & 107.5 & 4.6 & 46 & 820 & 3 FGL J2227.8+0040 \\
\hline P7R43479 & $22^{\mathrm{h}} 28^{\mathrm{m}} 35^{\mathrm{s}}$ & $-16^{\circ} 36^{\prime} 06^{\prime \prime}$ & 43.1 & -55.4 & 45 & 350 & 3FGL J2228.5-1636 \\
\hline P72Y3668 & $22^{\mathrm{h}} 50^{\mathrm{m}} 10^{\mathrm{s}}$ & $+33^{\circ} 06^{\prime} 01^{\prime \prime}$ & 95.5 & -23.2 & 40 & 350 & 3 FGL J2250.6+3308 \\
\hline P72Y3672 & $22^{\mathrm{h}} 50^{\mathrm{m}} 26^{\mathrm{s}}$ & $+17^{\circ} 50^{\prime} 42^{\prime \prime}$ & 86.4 & -36.3 & 44 & 820 & 3 FGL J2250.3+1747 \\
\hline 1FGL J2256.9-1024 & $22^{\mathrm{h}} 56^{\mathrm{m}} 52^{\mathrm{s}}$ & $-10^{\circ} 22^{\prime} 46^{\prime \prime}$ & 59.3 & -58.3 & 5 & 820 & None \\
\hline P72Y3714 ${ }^{\dagger}$ & $23^{\mathrm{h}} 10^{\mathrm{m}} 20^{\mathrm{s}}$ & $-05^{\circ} 46^{\prime} 29^{\prime \prime}$ & 70.0 & -57.8 & 40 & 350 & 3FGL J2310.1-0557 \\
\hline P72Y3714_0 & $23^{\mathrm{h}} 10^{\mathrm{m}} 20^{\mathrm{s}}$ & $-05^{\circ} 46^{\prime} 30^{\prime \prime}$ & 70.0 & -57.8 & 10 & 820 & 3FGL J2310.1-0557 \\
\hline P72Y3714_0 & $23^{\mathrm{h}} 10^{\mathrm{m}} 20^{\mathrm{s}}$ & $-05^{\circ} 46^{\prime} 30^{\prime \prime}$ & 70.0 & -57.8 & 10 & 820 & 3FGL J2310.1-0557 \\
\hline 1FGL J2339.7-0531 & $23^{\mathrm{h}} 39^{\mathrm{m}} 42^{\mathrm{s}}$ & $-05^{\circ} 31^{\prime} 17^{\prime \prime}$ & 81.4 & -62.5 & 32 & 820 & None \\
\hline 1FGL J2339.7-0531 & $23^{\mathrm{h}} 39^{\mathrm{m}} 42^{\mathrm{s}}$ & $-05^{\circ} 31^{\prime} 17^{\prime \prime}$ & 81.4 & -62.5 & 5 & 820 & None \\
\hline 1FGL J2339.7-0531 & $23^{\mathrm{h}} 39^{\mathrm{m}} 42^{\mathrm{s}}$ & $-05^{\circ} 31^{\prime} 17^{\prime \prime}$ & 81.4 & -62.5 & 5 & 820 & None \\
\hline P72Y3794 & $23^{\mathrm{h}} 45^{\mathrm{m}} 10^{\mathrm{s}}$ & $-15^{\circ} 55^{\prime} 16^{\prime \prime}$ & 65.6 & -71.0 & 45 & 820 & 3FGL J2345.2-1554 \\
\hline
\end{tabular}


Table 5.7. Radio Searches of Fermi Unassociated Sources near the Galactic Plane

\begin{tabular}{|c|c|c|c|c|c|c|c|}
\hline Source & $\begin{array}{c}\text { R.A. } \\
\text { J2000.0 }\end{array}$ & $\begin{array}{c}\text { Decl. } \\
\text { J2000.0 }\end{array}$ & $\begin{array}{c}l \\
\operatorname{deg}\end{array}$ & $\begin{array}{c}b \\
\operatorname{deg}\end{array}$ & $\begin{array}{l}\text { Integra- } \\
\text { tion Time } \\
\text { minutes }\end{array}$ & $\begin{array}{c}\text { Observing } \\
\text { Freq } \\
\mathrm{MHz}\end{array}$ & 3FGL name \\
\hline P72Y0008 & $00^{\mathrm{h}} 02^{\mathrm{m}} 40^{\mathrm{s}}$ & $+62^{\circ} 16^{\prime} 44^{\prime \prime}$ & 117.3 & -0.1 & 27 & 2000 & 3FGL J0002.6+6218 \\
\hline P72Y0106_0 & $00^{\mathrm{h}} 38^{\mathrm{m}} 51^{\mathrm{s}}$ & $+63^{\circ} 00^{\prime} 32^{\prime \prime}$ & 121.5 & 0.2 & 8 & 2000 & 3FGL J0039.3+6256 \\
\hline $\mathrm{P} 72 \mathrm{Y} 0348$ & $02^{\mathrm{h}} 23^{\mathrm{m}} 53^{\mathrm{s}}$ & $+62^{\circ} 04^{\prime} 59^{\prime \prime}$ & 133.5 & 1.1 & 26 & 2000 & 3FGL J0223.6+6204 \\
\hline 2FGL J0224.0+6204 & $02^{\mathrm{h}} 24^{\mathrm{m}} 05^{\mathrm{s}}$ & $+62^{\circ} 04^{\prime} 35^{\prime \prime}$ & 133.5 & 1.1 & 59 & 2000 & 3FGL J0223.6+6204 \\
\hline P72Y0352_0 & $02^{\mathrm{h}} 26^{\mathrm{m}} 03^{\mathrm{s}}$ & $+61^{\circ} 55^{\prime} 48^{\prime \prime}$ & 133.8 & 1.1 & 10 & 2000 & None \\
\hline 2FGL J0359.5+5410 & $03^{\mathrm{h}} 59^{\mathrm{m}} 33^{\mathrm{s}}$ & $+54^{\circ} 10^{\prime} 49^{\prime \prime}$ & 148.3 & 0.8 & 32 & 2000 & 3FGL J0359.5+5413 \\
\hline P72Y0624_0 & $04^{\mathrm{h}} 27^{\mathrm{m}} 07^{\mathrm{s}}$ & $+54^{\circ} 36^{\prime} 58^{\prime \prime}$ & 150.9 & 3.9 & 8 & 2000 & 3FGL J0426.7+5437 \\
\hline P72Y0896_0 & $06^{\mathrm{h}} 08^{\mathrm{m}} 18^{\mathrm{s}}$ & $+20^{\circ} 37^{\prime} 07^{\prime \prime}$ & 189.8 & 0.3 & 7 & 2000 & 3 FGL J0609.2+2051c \\
\hline $\mathrm{P} 72 \mathrm{Y} 1310$ & $09^{\mathrm{h}} 01^{\mathrm{m}} 36^{\mathrm{s}}$ & $+54^{\circ} 12^{\prime} 57^{\prime \prime}$ & 163.4 & 40.5 & 53 & 2000 & None \\
\hline P72Y2550_0 & $16^{\mathrm{h}} 36^{\mathrm{m}} 10^{\mathrm{s}}$ & $+54^{\circ} 12^{\prime} 56^{\prime \prime}$ & 82.7 & 41.3 & 7 & 2000 & None \\
\hline $\mathrm{P} 72 \mathrm{Y} 2604$ & $16^{\mathrm{h}} 53^{\mathrm{m}} 35^{\mathrm{s}}$ & $-01^{\circ} 59^{\prime} 24^{\prime \prime}$ & 16.6 & 24.9 & 50 & 2000 & 3FGL J1653.6-0158 \\
\hline J1803.1-2147c & $18^{\mathrm{h}} 03^{\mathrm{m}} 11^{\mathrm{s}}$ & $-21^{\circ} 47^{\prime} 27^{\prime \prime}$ & 8.2 & 0.2 & 67 & 2000 & 3FGL J1803.1-2147 \\
\hline P72Y2994 & $18^{\mathrm{h}} 39^{\mathrm{m}} 15^{\mathrm{s}}$ & $-05^{\circ} 56^{\prime} 46^{\prime \prime}$ & 26.3 & -0.0 & 40 & 2000 & 3FGL J1838.9-0537 \\
\hline 2FGL J1844.3-0343c & $18^{\mathrm{h}} 44^{\mathrm{m}} 23^{\mathrm{s}}$ & $-03^{\circ} 43^{\prime} 54^{\prime \prime}$ & 28.8 & -0.1 & 21 & 2000 & 3FGL J1844.3-0344 \\
\hline 2FGL J1857.2+0055c & $18^{\mathrm{h}} 57^{\mathrm{m}} 14^{\mathrm{s}}$ & $-03^{\circ} 43^{\prime} 54^{\prime \prime}$ & 30.3 & -3.0 & 27 & 2000 & 3FGL J1857.2+0059 \\
\hline P72Y3053 & $18^{\mathrm{h}} 57^{\mathrm{m}} 41^{\mathrm{s}}$ & $+02^{\circ} 12^{\prime} 45^{\prime \prime}$ & 35.6 & -0.4 & 43 & 2000 & 3FGL J1857.9+0210 \\
\hline 2FGL J1906.5+0720 & $19^{\mathrm{h}} 06^{\mathrm{m}} 35^{\mathrm{s}}$ & $+07^{\circ} 20^{\prime} 33^{\prime \prime}$ & 41.2 & -0.0 & 27 & 2000 & 3FGL J1906.6+0720 \\
\hline P72Y3081_0 & $19^{\mathrm{h}} 06^{\mathrm{m}} 38^{\mathrm{s}}$ & $+07^{\circ} 19^{\prime} 19^{\prime \prime}$ & 41.2 & -0.0 & 7 & 2000 & 3FGL J1906.6+0720 \\
\hline 2FGL J2034.9+3632 & $20^{\mathrm{h}} 34^{\mathrm{m}} 57^{\mathrm{s}}$ & $+36^{\circ} 32^{\prime} 04^{\prime \prime}$ & 76.6 & -2.3 & 23 & 2000 & 3FGL J2035.0+3634 \\
\hline P72Y3325_0 & $20^{\mathrm{h}} 35^{\mathrm{m}} 00^{\mathrm{s}}$ & $+36^{\circ} 33^{\prime} 37^{\prime \prime}$ & 76.6 & -2.3 & 7 & 2000 & 3FGL J2035.0+3634 \\
\hline
\end{tabular}




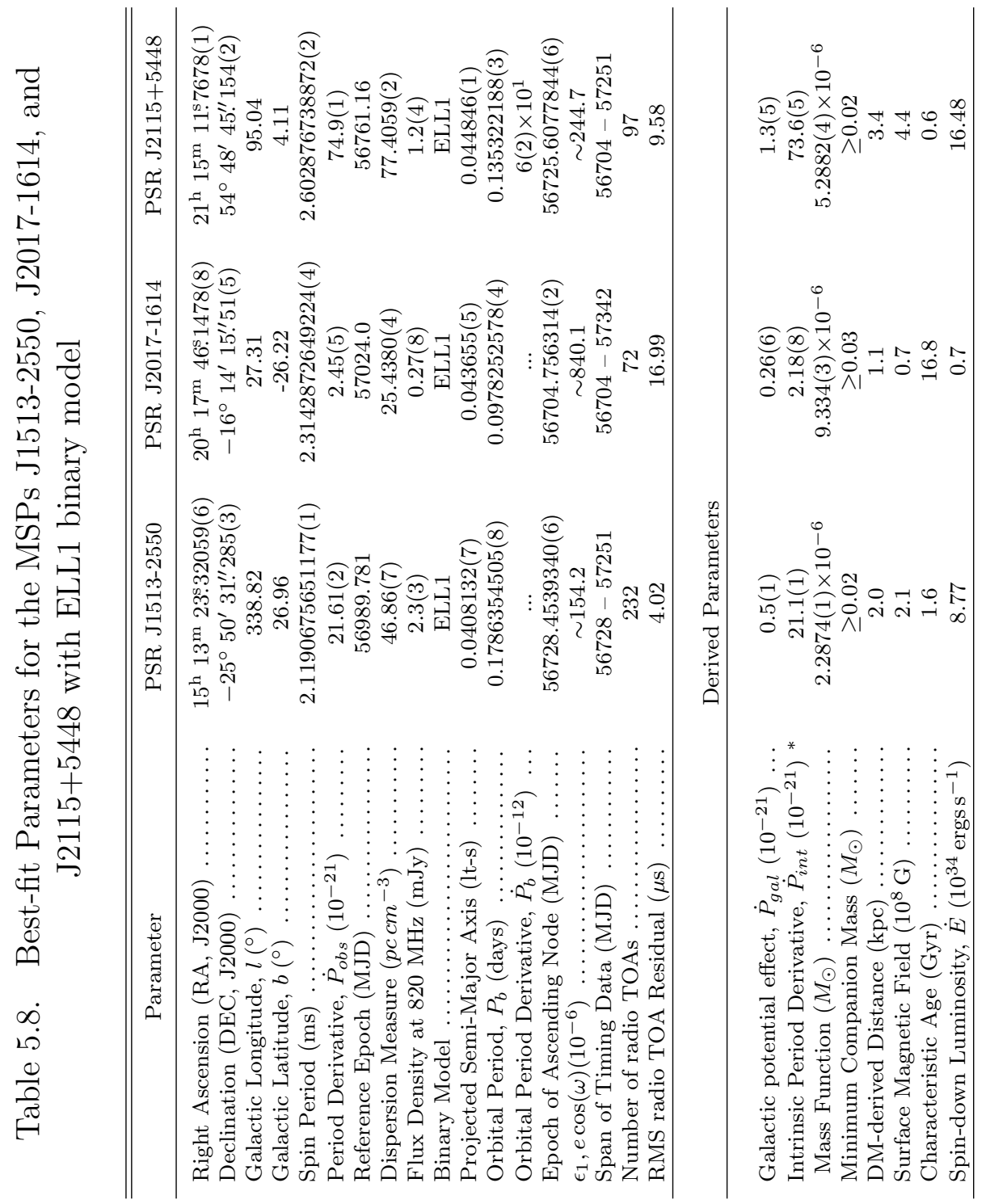




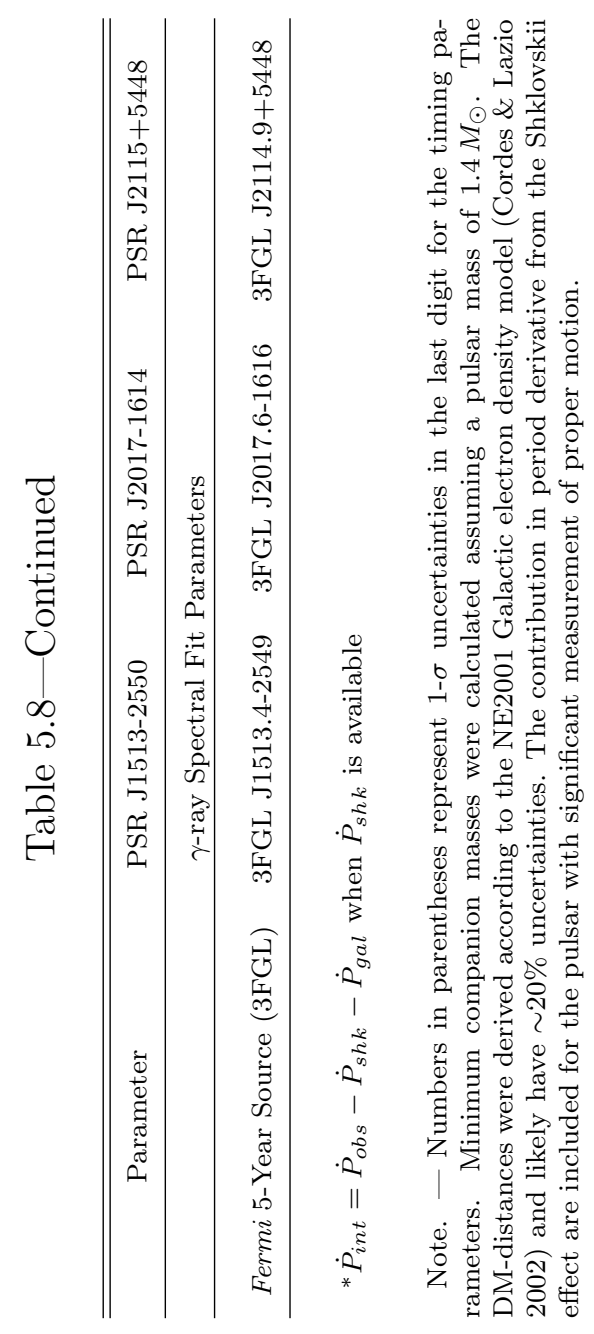


Table 5.9. Best-fit Parameters for the MSP J1622-0315 with BTX binary model

\begin{tabular}{|c|c|}
\hline Parameter & PSR J1622-0315 \\
\hline Right Ascension (RA, J2000) & $16^{\mathrm{h}} 22^{\mathrm{m}} 59.6285(3)$ \\
\hline Declination (DEC, J2000) & $-03^{\circ} 15^{\prime} 37^{\prime \prime} \cdot 328(7)$ \\
\hline Galactic Longitude, $l\left(^{\circ}\right)$ & 10.71 \\
\hline Galactic Latitude, $b\left(^{\circ}\right)$. & 30.68 \\
\hline Spin Period $(\mathrm{ms}) \ldots .$. & $3.845429067931(3)$ \\
\hline Period Derivative, $\dot{P}_{o b s}\left(10^{-21}\right)$ & $11.6(1)$ \\
\hline Reference Epoch (MJD) .... & 56769.0 \\
\hline Dispersion Measure $\left(p c c^{-3}\right)$ & 21.4 \\
\hline Flux Density at $820 \mathrm{MHz}$ (mJy) & $1(2)$ \\
\hline Binary Model & BTX \\
\hline Projected Semi-Major Axis (lt-s) & $0.219258(5)$ \\
\hline Orbital Period, $P_{b}$ (days) $\ldots$ & $0.1617006798(6)$ \\
\hline Orbital Period Derivative, $\dot{P}_{b}\left(10^{-12}\right)$ & $-4.1(2) \times 10^{-9}$ \\
\hline Orbital Frequency, $F_{b}(\mathrm{~Hz}) \ldots \ldots \ldots \ldots$ & $7.15771516(3) \times 10^{-5}$ \\
\hline Orbital Frequency Derivative, $\dot{F}_{b}\left(\mathrm{~Hz}^{-1}\right)$ & $1.57(7) \times 10^{-19}$ \\
\hline Epoch of Periastron (MJD) $\ldots \ldots \ldots \ldots$ & $56733.668703(1)$ \\
\hline Span of Timing Data (MJD) $\ldots \ldots \ldots \ldots$ & $56733-57549$ \\
\hline Number of radio TOAs $\ldots \ldots \ldots \ldots \ldots \ldots$ & 268 \\
\hline RMS radio TOA Residual $(\mu \mathrm{s}) \ldots \ldots \ldots$ & 17.21 \\
\hline
\end{tabular}

Derived Parameters

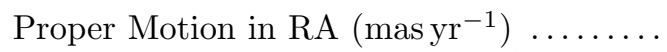

$-15.4(1.7)$

Proper Motion in DEC $\left(\operatorname{mas~yr}^{-1}\right) \ldots \ldots$. Transverse velocity, $V_{T}\left(\mathrm{~km} \mathrm{~s}^{-1}\right) \ldots \ldots \ldots$ Shklovskii effect, $\dot{P}_{s h k}\left(10^{-21}\right) \ldots \ldots \ldots \ldots$ Galactic potential effect, $\dot{P}_{g a l}\left(10^{-21}\right) \ldots$ Intrinsic Period Derivative, $\dot{P}_{\text {int }}\left(10^{-21}\right) *$. Mass Function $\left(M_{\odot}\right) \ldots \ldots \ldots \ldots \ldots$ Minimum Companion Mass $\left(M_{\odot}\right) \ldots \ldots$. DM-derived Distance (kpc) .............. Surface Magnetic Field $\left(10^{8} \mathrm{G}\right) \ldots \ldots \ldots$ Characteristic Age (Gyr) ..............
$37.5(5.1)$

$2.1(5) \times 10^{2}$

$17(5)$

$0.4(1)$

$11.1(1)$

$0.00043284(3)$

$\geq 0.1$

1.1

2.1

5.5 
Table 5.9-Continued

\begin{tabular}{|c|c|}
\hline Parameter & PSR J1622-0315 \\
\hline Spin-down Luminosity, $\dot{E}\left(10^{34} \operatorname{ergs~s}^{-1}\right)$ & 0.77 \\
\hline \multicolumn{2}{|c|}{$\gamma$-ray Spectral Fit Parameters } \\
\hline Fermi 5 -Year Source $(3 F G L) \ldots \ldots \ldots \ldots$ & 3FGL J1622.9-0312 \\
\hline \multicolumn{2}{|c|}{${ }^{*} \dot{P}_{i n t}=\dot{P}_{o b s}-\dot{P}_{s h k}-\dot{P}_{g a l}$ when $\dot{P}_{s h k}$ is available } \\
\hline $\begin{array}{l}\text { Note. - Numbers in parentheses repr } \\
\text { in the last digit for the timing parameter } \\
\text { ion masses were calculated assuming a p } \\
\text { The DM-distances were derived according } \\
\text { tic electron density model (Cordes \& Lazi } \\
\sim 20 \% \text { uncertainties. The contribution in } \\
\text { the Shklovskii effect are included for the } \\
\text { measurement of proper motion. }\end{array}$ & $\begin{array}{l}\text { sent } 1-\sigma \text { uncertainties } \\
\text { s. Minimum compan- } \\
\text { ulsar mass of } 1.4 M_{\odot} \text {. } \\
\text { to the NE2001 Galac- } \\
2002) \text { and likely have } \\
\text { period derivative from } \\
\text { pulsar with significant }\end{array}$ \\
\hline
\end{tabular}




\section{References}

Abbott, B. P., Abbott, R., Abbott, T. D., Abernathy, M. R., Acernese, F., Ackley, K., Adams, C., Adams, T., Addesso, P., Adhikari, R. X., \& et al. 2016a, Physical Review Letters, 116, 241103

—. 2016b, Physical Review Letters, 116, 061102

Abdo, A. A., Ackermann, M., Ajello, M., Allafort, A., Antolini, E., Atwood, W. B., Axelsson, M., Baldini, L., Ballet, J., Barbiellini, G., \& et al. 2010a, ApJS, 188, 405

Abdo, A. A., Ackermann, M., Ajello, M., Anderson, B., Atwood, W. B., Axelsson, M., Baldini, L., Ballet, J., Barbiellini, G., Baring, M. G., Bastieri, D., Baughman, B. M., Bechtol, K., Bellazzini, R., Berenji, B., Bignami, G. F., Blandford, R. D., Bloom, E. D., Bonamente, E., Borgland, A. W., Bregeon, J., Brez, A., Brigida, M., Bruel, P., Burnett, T. H., Caliandro, G. A., Cameron, R. A., Caraveo, P. A., Casandjian, J. M., Cecchi, C., Çelik, Ö., Chekhtman, A., Cheung, C. C., Chiang, J., Ciprini, S., Claus, R., Cohen-Tanugi, J., Conrad, J., Cutini, S., Dermer, C. D., de Angelis, A., de Luca, A., de Palma, F., Digel, S. W., Dormody, M., do Couto e Silva, E., Drell, P. S., Dubois, R., Dumora, D., Farnier, C., Favuzzi, C., Fegan, S. J., Fukazawa, Y., Funk, S., Fusco, P., Gargano, F., Gasparrini, D., Gehrels, N., Germani, S., Giebels, B., Giglietto, N., Giommi, P., Giordano, F., Glanzman, T., Godfrey, G., Grenier, I. A., Grondin, M.-H., Grove, J. E., Guillemot, L., Guiriec, 
S., Gwon, C., Hanabata, Y., Harding, A. K., Hayashida, M., Hays, E., Hughes, R. E., Jóhannesson, G., Johnson, R. P., Johnson, T. J., Johnson, W. N., Kamae, T., Katagiri, H., Kataoka, J., Kawai, N., Kerr, M., Knödlseder, J., Kocian, M. L., Kuss, M., Lande, J., Latronico, L., Lemoine-Goumard, M., Longo, F., Loparco, F., Lott, B., Lovellette, M. N., Lubrano, P., Madejski, G. M., Makeev, A., Marelli, M., Mazziotta, M. N., McConville, W., McEnery, J. E., Meurer, C., Michelson, P. F., Mitthumsiri, W., Mizuno, T., Monte, C., Monzani, M. E., Morselli, A., Moskalenko, I. V., Murgia, S., Nolan, P. L., Norris, J. P., Nuss, E., Ohsugi, T., Omodei, N., Orlando, E., Ormes, J. F., Paneque, D., Parent, D., Pelassa, V., Pepe, M., PesceRollins, M., Pierbattista, M., Piron, F., Porter, T. A., Primack, J. R., Rainò, S., Rando, R., Ray, P. S., Razzano, M., Rea, N., Reimer, A., Reimer, O., Reposeur, T., Ritz, S., Rochester, L. S., Rodriguez, A. Y., Romani, R. W., Ryde, F., Sadrozinski, H. F.-W., Sanchez, D., Sander, A., Parkinson, P. M. S., Scargle, J. D., Sgrò, C., Siskind, E. J., Smith, D. A., Smith, P. D., Spandre, G., Spinelli, P., Starck, J.-L., Strickman, M. S., Suson, D. J., Tajima, H., Takahashi, H., Takahashi, T., Tanaka, T., Thayer, J. G., Thompson, D. J., Tibaldo, L., Tibolla, O., Torres, D. F., Tosti, G., Tramacere, A., Uchiyama, Y., Usher, T. L., Van Etten, A., Vasileiou, V., Vilchez, N., Vitale, V., Waite, A. P., Wang, P., Watters, K., Winer, B. L., Wolff, M. T., Wood, K. S., Ylinen, T., Ziegler, M., \& Fermi LAT Collaboration. 2009a, Science, 325, 840

Abdo, A. A., Ackermann, M., Ajello, M., Atwood, W. B., Axelsson, M., Baldini, L., Ballet, J., Band, D. L., Barbiellini, G., Bastieri, D., \& et al. 2009b, ApJS, 183, 46

Abdo, A. A., Ackermann, M., Ajello, M., Atwood, W. B., Axelsson, M., Baldini, L., Ballet, J., Barbiellini, G., Baring, M. G., Bastieri, D., \& et al. 2010b, ApJS, 187, 460 
Abdo, A. A., Ackermann, M., Atwood, W. B., Baldini, L., Ballet, J., Barbiellini, G., Baring, M. G., Bastieri, D., \& others. 2009c, Astroparticle Physics, 32, 193

Abdo, A. A., Ajello, M., Allafort, A., Baldini, L., Ballet, J., Barbiellini, G., Baring, M. G., Bastieri, D., Belfiore, A., Bellazzini, R., \& et al. 2013, ApJS, 208, 17

Acero, F., Ackermann, M., Ajello, M., Albert, A., Atwood, W. B., Axelsson, M., Baldini, L., Ballet, J., Barbiellini, G., Bastieri, D., Belfiore, A., Bellazzini, R., Bissaldi, E., Blandford, R. D., Bloom, E. D., Bogart, J. R., Bonino, R., Bottacini, E., Bregeon, J., Britto, R. J., Bruel, P., Buehler, R., Burnett, T. H., Buson, S., Caliandro, G. A., Cameron, R. A., Caputo, R., Caragiulo, M., Caraveo, P. A., Casandjian, J. M., Cavazzuti, E., Charles, E., Chaves, R. C. G., Chekhtman, A., Cheung, C. C., Chiang, J., Chiaro, G., Ciprini, S., Claus, R., Cohen-Tanugi, J., Cominsky, L. R., Conrad, J., Cutini, S., D’Ammando, F., de Angelis, A., DeKlotz, M., de Palma, F., Desiante, R., Digel, S. W., Di Venere, L., Drell, P. S., Dubois, R., Dumora, D., Favuzzi, C., Fegan, S. J., Ferrara, E. C., Finke, J., Franckowiak, A., Fukazawa, Y., Funk, S., Fusco, P., Gargano, F., Gasparrini, D., Giebels, B., Giglietto, N., Giommi, P., Giordano, F., Giroletti, M., Glanzman, T., Godfrey, G., Grenier, I. A., Grondin, M.-H., Grove, J. E., Guillemot, L., Guiriec, S., Hadasch, D., Harding, A. K., Hays, E., Hewitt, J. W., Hill, A. B., Horan, D., Iafrate, G., Jogler, T., Jóhannesson, G., Johnson, R. P., Johnson, A. S., Johnson, T. J., Johnson, W. N., Kamae, T., Kataoka, J., Katsuta, J., Kuss, M., La Mura, G., Landriu, D., Larsson, S., Latronico, L., Lemoine-Goumard, M., Li, J., Li, L., Longo, F., Loparco, F., Lott, B., Lovellette, M. N., Lubrano, P., Madejski, G. M., Massaro, F., Mayer, M., Mazziotta, M. N., McEnery, J. E., Michelson, P. F., Mirabal, N., Mizuno, T., Moiseev, A. A., Mongelli, M., Monzani, M. E., Morselli, A., Moskalenko, I. V., Murgia, S., Nuss, E., Ohno, M., Ohsugi, T., Omodei, N., Orienti, M., Orlando, E., 
Ormes, J. F., Paneque, D., Panetta, J. H., Perkins, J. S., Pesce-Rollins, M., Piron, F., Pivato, G., Porter, T. A., Racusin, J. L., Rando, R., Razzano, M., Razzaque, S., Reimer, A., Reimer, O., Reposeur, T., Rochester, L. S., Romani, R. W., Salvetti, D., Sánchez-Conde, M., Saz Parkinson, P. M., Schulz, A., Siskind, E. J., Smith, D. A., Spada, F., Spandre, G., Spinelli, P., Stephens, T. E., Strong, A. W., Suson, D. J., Takahashi, H., Takahashi, T., Tanaka, Y., Thayer, J. G., Thayer, J. B., Thompson, D. J., Tibaldo, L., Tibolla, O., Torres, D. F., Torresi, E., Tosti, G., Troja, E., Van Klaveren, B., Vianello, G., Winer, B. L., Wood, K. S., Wood, M., Zimmer, S., \& Fermi-LAT Collaboration. 2015, ApJS, 218, 23

Ackermann, M., Ajello, M., Albert, A., Allafort, A., Atwood, W. B., Axelsson, M., Baldini, L., et al. 2012, ApJS, 203, 4

Alpar, M. A., Cheng, A. F., Ruderman, M. A., \& Shaham, J. 1982, Nature, 300, 728 Archibald, A. M., Bogdanov, S., Patruno, A., Hessels, J. W. T., Deller, A. T., Bassa, C., Janssen, G. H., Kaspi, V. M., Lyne, A. G., Stappers, B. W., Tendulkar, S. P., D’Angelo, C. R., \& Wijnands, R. 2015, ApJ, 807, 62

Archibald, A. M., Kaspi, V. M., Bogdanov, S., Hessels, J. W. T., Stairs, I. H., Ransom, S. M., \& McLaughlin, M. A. 2010, ApJ, 722, 88

Archibald, A. M., Stairs, I. H., Ransom, S. M., Kaspi, V. M., Kondratiev, V. I., Lorimer, D. R., McLaughlin, M. A., Boyles, J., Hessels, J. W. T., Lynch, R., van Leeuwen, J., Roberts, M. S. E., Jenet, F., Champion, D. J., Rosen, R., Barlow, B. N., Dunlap, B. H., \& Remillard, R. A. 2009, Science, 324, 1411

Atwood, W. B., Abdo, A. A., Ackermann, M., Althouse, W., Anderson, B., Axelsson, 
M., Baldini, L., Ballet, J., Band, D. L., Barbiellini, G., \& et al. 2009, ApJ, 697, 1071

Baade, W. \& Zwicky, F. 1934, Proceedings of the National Academy of Science, 20, 259

Barr, E. D., Guillemot, L., Champion, D. J., Kramer, M., Eatough, R. P., Lee, K. J., Verbiest, J. P. W., et al. 2013, MNRAS, 429, 1633

Bates, S. D., Lorimer, D. R., \& Verbiest, J. P. W. 2013, MNRAS, 431, 1352

Bellm, E. C., Kaplan, D. L., Breton, R. P., Phinney, E. S., Bhalerao, V. B., Camilo, F., Dahal, S., Djorgovski, S. G., Drake, A. J., Hessels, J. W. T., Laher, R. R., Levitan, D. B., Lewis, F., Mahabal, A. A., Ofek, E. O., Prince, T. A., Ransom, S. M., Roberts, M. S. E., Russell, D. M., Sesar, B., Surace, J. A., \& Tang, S. 2016, ApJ, 816, 74

Benvenuto, O. G., De Vito, M. A., \& Horvath, J. E. 2015, ApJ, 798, 44

Bhattacharyya, B., Roy, J., Ray, P. S., Gupta, Y., Bhattacharya, D., Romani, R. W., Ransom, S. M., et al. 2013, ApJ, 773, L12

Bochenek, C., Ransom, S., \& Demorest, P. 2015, ApJ, 813, L4

Bregeon, J., Charles, E., \& M. Wood for the Fermi-LAT collaboration. 2013, in Proceedings of the 4th Fermi Symposium, eConf C121028, (arXiv:1304.5456)

Burke-Spolaor, S., Johnston, S., Bailes, M., Bates, S. D., Bhat, N. D. R., Burgay, M., Champion, D. J., D’Amico, N., Keith, M. J., Kramer, M., Levin, L., Milia, S., Possenti, A., Stappers, B., \& van Straten, W. 2012, MNRAS, 423, 1351 
Camilo, F., Kerr, M., Ray, P. S., Ransom, S. M., Sarkissian, J., Cromartie, H. T., Johnston, S., Reynolds, J. E., Wolff, M. T., Freire, P. C. C., Bhattacharyya, B., Ferrara, E. C., Keith, M., Michelson, P. F., Saz Parkinson, P. M., \& Wood, K. S. 2015, ArXiv e-prints, 1507.04451

Chakrabarty, D. \& Morgan, E. H. 1998, Nature, 394, 346

Champion, D. J., McLaughlin, M. A., \& Lorimer, D. R. 2005, MNRAS, 364, 1011

Chen, H.-L., Chen, X., Tauris, T. M., \& Han, Z. 2013, ApJ, 775, 27

Cheng, K. S., Ho, C., \& Ruderman, M. 1986, ApJ, 300, 500

Cognard, I., Guillemot, L., Johnson, T. J., Smith, D. A., Venter, C., Harding, A. K., Wolff, M. T., et al. 2011, ApJ, 732, 47

Cordes, J. M. \& Lazio, T. J. W. 2002, ArXiv Astrophysics e-prints, astro-ph/0207156

Crawford, F., Roberts, M. S. E., Hessels, J. W. T., Ransom, S. M., Livingstone, M., Tam, C. R., \& Kaspi, V. M. 2006, ApJ, 652, 1499

Cromartie, H. T., Camilo, F., Kerr, M., Deneva, J. S., Ransom, S. M., Ray, P. S., Ferrara, E. C., Michelson, P. F., \& Wood, K. S. 2016, ApJ, 819, 34

Damour, T. \& Deruelle, N. 1986, Ann. Inst. Henri Poincaré Phys. Théor., Vol. 44, No. 3, p. $263-292,44,263$

Demorest, P. B., Pennucci, T., Ransom, S. M., Roberts, M. S. E., \& Hessels, J. W. T. 2010, Nature, 467, 1081

Deneva, J. S., Cordes, J. M., McLaughlin, M. A., Nice, D. J., Lorimer, D. R., Crawford, F., Bhat, N. D. R., et al. 2009, ApJ, 703, 2259 
Dubus, G. 2015, Comptes Rendus Physique, 16, 661

DuPlain, R., Ransom, S., Demorest, P., Brandt, P., Ford, J., \& Shelton, A. L. 2008, in Society of Photo-Optical Instrumentation Engineers (SPIE) Conference Series, Vol. 7019, Society of Photo-Optical Instrumentation Engineers (SPIE) Conference Series, 1

Foreman-Mackey, D., Hogg, D. W., Lang, D., \& Goodman, J. 2013, PASP, 125, 306

Freire, P. C. C., Abdo, A. A., Ajello, M., Allafort, A., Ballet, J., Barbiellini, G., Bastieri, D., et al. 2011, Science, 334, 1107

Fruchter, A. S., Stinebring, D. R., \& Taylor, J. H. 1988, Nature, 333, 237

Grenier, I. A. \& Harding, A. K. 2015, Comptes Rendus Physique, 16, 641

Guillemot, L., Johnson, T. J., Venter, C., Kerr, M., Pancrazi, B., Livingstone, M., Janssen, G. H., et al. 2012, ApJ, 744, 33

Guillemot, L. \& Tauris, T. M. 2014, MNRAS, 439, 2033

Hessels, J. W. T., Ransom, S. M., Stairs, I. H., Kaspi, V. M., \& Freire, P. C. C. 2007, ApJ, 670, 363

Hewish, A., Bell, S. J., Pilkington, J. D. H., Scott, P. F., \& Collins, R. A. 1968, Nature, 217, 709

Hobbs, G., Archibald, A., Arzoumanian, Z., Backer, D., Bailes, M., Bhat, N. D. R., Burgay, M., Burke-Spolaor, S., Champion, D., Cognard, I., Coles, W., Cordes, J., Demorest, P., Desvignes, G., Ferdman, R. D., Finn, L., Freire, P., Gonzalez, M., Hessels, J., Hotan, A., Janssen, G., Jenet, F., Jessner, A., Jordan, C., Kaspi, V., Kramer, M., Kondratiev, V., Lazio, J., Lazaridis, K., Lee, K. J., Levin, Y., 
Lommen, A., Lorimer, D., Lynch, R., Lyne, A., Manchester, R., McLaughlin, M., Nice, D., Oslowski, S., Pilia, M., Possenti, A., Purver, M., Ransom, S., Reynolds, J., Sanidas, S., Sarkissian, J., Sesana, A., Shannon, R., Siemens, X., Stairs, I., Stappers, B., Stinebring, D., Theureau, G., van Haasteren, R., van Straten, W., Verbiest, J. P. W., Yardley, D. R. B., \& You, X. P. 2010, Classical and Quantum Gravity, 27, 084013

Hobbs, G. B., Edwards, R. T., \& Manchester, R. N. 2006, MNRAS, 369, 655

Huang, R. H. H., Kong, A. K. H., Takata, J., Hui, C. Y., Lin, L. C. C., \& Cheng, K. S. 2012, ApJ, 760, 92

Jacoby, B. A., Hotan, A., Bailes, M., Ord, S., \& Kulkarni, S. R. 2005, ApJ, 629, L113

Johnson, T. J., Venter, C., Harding, A. K., Guillemot, L., Smith, D. A., Kramer, M., Çelik, Ö., den Hartog, P. R., Ferrara, E. C., Hou, X., Lande, J., \& Ray, P. S. 2014, ApJS, 213, 6

Johnston, S., Manchester, R. N., Lyne, A. G., Bailes, M., Kaspi, V. M., Qiao, G., \& D’Amico, N. 1992, ApJ, 387, L37

Johnston, S. \& Romani, R. W. 2003, ApJ, 590, L95

Joss, P. C., Rappaport, S., \& Lewis, W. 1987, ApJ, 319, 180

Kaplan, D. L., van Kerkwijk, M. H., Koester, D., Stairs, I. H., Ransom, S. M., Archibald, A. M., Hessels, J. W. T., \& Boyles, J. 2014, ApJ, 783, L23

Keith, M. J., Johnston, S., Kramer, M., Weltevrede, P., Watters, K. P., \& Stappers, B. W. 2008, MNRAS, 389, 1881 
Keith, M. J., Johnston, S., Ray, P. S., Ferrara, E. C., Saz Parkinson, P. M., Çelik, Ö., Belfiore, A., et al. 2011, MNRAS, 414, 1292

Kerr, M. 2011, ApJ, 732, 38

Kerr, M., Camilo, F., Johnson, T. J., Ferrara, E. C., Guillemot, L., Harding, A. K., Hessels, J., et al. 2012, ApJ, 748, L2

Knight, H. S. 2006, Chinese Journal of Astronomy and Astrophysics Supplement, 6, 41

Kramer, M., Lange, C., Lorimer, D. R., Backer, D. C., Xilouris, K. M., Jessner, A., \& Wielebinski, R. 1999, ApJ, 526, 957

Kramer, M., Stairs, I. H., Manchester, R. N., McLaughlin, M. A., Lyne, A. G., Ferdman, R. D., Burgay, M., Lorimer, D. R., Possenti, A., D’Amico, N., Sarkissian, J. M., Hobbs, G. B., Reynolds, J. E., Freire, P. C. C., \& Camilo, F. 2006, Science, 314,97

Kramer, M., Xilouris, K. M., Lorimer, D. R., Doroshenko, O., Jessner, A., Wielebinski, R., Wolszczan, A., \& Camilo, F. 1998, ApJ, 501, 270

Lange, C., Camilo, F., Wex, N., Kramer, M., Backer, D. C., Lyne, A. G., \& Doroshenko, O. 2001, MNRAS, 326, 274

Lawson, K. D., Mayer, C. J., Osborne, J. L., \& Parkinson, M. L. 1987, MNRAS, 225, 307

Li, M., Halpern, J. P., \& Thorstensen, J. R. 2014, ApJ, 795, 115 
Lorimer, D. \& Kramer, M. 2005, Cambridge Observing Handbooks for Research Astronomers, Vol. 4, Handbook of Pulsar Astronomy, 1st edn. (Cambridge, U.K.; New York, U.S.A: Cambridge University Press)

Lundgren, S. C., Zepka, A. F., \& Cordes, J. M. 1995, ApJ, 453, 419

Lynch, R. S. \& Bank North Celestial Cap Survey Collaborations. 2013, in IAU Symposium, Vol. 291, Neutron Stars and Pulsars: Challenges and Opportunities after 80 years, ed. J. van Leeuwen, 41-46

Lyutikov, M., Otte, N., \& McCann, A. 2012, ApJ, 754, 33

Magro, A., Karastergiou, A., Salvini, S., Mort, B., Dulwich, F., \& Zarb Adami, K. 2011, MNRAS, 417, 2642

Manchester, R. N., Hobbs, G. B., Teoh, A., \& Hobbs, M. 2005, VizieR Online Data Catalog, 7245, 0

McLaughlin, M. A., Lyne, A. G., Lorimer, D. R., Kramer, M., Faulkner, A. J., Manchester, R. N., Cordes, J. M., Camilo, F., Possenti, A., Stairs, I. H., Hobbs, G., D’Amico, N., Burgay, M., \& O’Brien, J. T. 2006, Nature, 439, 817

Muslimov, A. G. \& Harding, A. K. 2004, ApJ, 606, 1143

Nolan, P. L., Abdo, A. A., Ackermann, M., Ajello, M., Allafort, A., Antolini, E., Atwood, W. B., Axelsson, M., Baldini, L., Ballet, J., \& et al. 2012, ApJS, 199, 31 Özel, F., Psaltis, D., Narayan, R., \& Santos Villarreal, A. 2012, ApJ, 757, 55

Papitto, A., Ferrigno, C., Bozzo, E., Rea, N., Pavan, L., Burderi, L., Burgay, M., Campana, S., di Salvo, T., Falanga, M., Filipović, M. D., Freire, P. C. C., Hessels, J. W. T., Possenti, A., Ransom, S. M., Riggio, A., Romano, P., Sarkissian, J. M., 
Stairs, I. H., Stella, L., Torres, D. F., Wieringa, M. H., \& Wong, G. F. 2013, Nature, 501,517

Pétri, J. 2012, in Astronomical Society of the Pacific Conference Series, Vol. 466, Electromagnetic Radiation from Pulsars and Magnetars, ed. W. Lewandowski, O. Maron, \& J. Kijak, 21

Pletsch, H. J. \& Clark, C. J. 2015, ApJ, 807, 18

Pletsch, H. J., Guillemot, L., Allen, B., Kramer, M., Aulbert, C., Fehrmann, H., Ray, P. S., Barr, E. D., Belfiore, A., Camilo, F., Caraveo, P. A., Çelik, Ö., Champion, D. J., Dormody, M., Eatough, R. P., Ferrara, E. C., Freire, P. C. C., Hessels, J. W. T., Keith, M., Kerr, M., de Luca, A., Lyne, A. G., Marelli, M., McLaughlin, M. A., Parent, D., Ransom, S. M., Razzano, M., Reich, W., Saz Parkinson, P. M., Stappers, B. W., \& Wolff, M. T. 2012a, ApJ, 744, 105

Pletsch, H. J., Guillemot, L., Fehrmann, H., Allen, B., Kramer, M., Aulbert, C., Ackermann, M., Ajello, M., de Angelis, A., Atwood, W. B., Baldini, L., Ballet, J., Barbiellini, G., Bastieri, D., Bechtol, K., Bellazzini, R., Borgland, A. W., Bottacini, E., Brandt, T. J., Bregeon, J., Brigida, M., Bruel, P., Buehler, R., Buson, S., Caliandro, G. A., Cameron, R. A., Caraveo, P. A., Casandjian, J. M., Cecchi, C., Çelik, Ö., Charles, E., Chaves, R. C. G., Cheung, C. C., Chiang, J., Ciprini, S., Claus, R., Cohen-Tanugi, J., Conrad, J., Cutini, S., D’Ammando, F., Dermer, C. D., Digel, S. W., Drell, P. S., Drlica-Wagner, A., Dubois, R., Dumora, D., Favuzzi, C., Ferrara, E. C., Franckowiak, A., Fukazawa, Y., Fusco, P., Gargano, F., Gehrels, N., Germani, S., Giglietto, N., Giordano, F., Giroletti, M., Godfrey, G., Grenier, I. A., Grondin, M.-H., Grove, J. E., Guiriec, S., Hadasch, D., Hanabata, Y., Harding, A. K., den Hartog, P. R., Hayashida, M., Hays, E., Hill, A. B., Hou, X., Hughes, 
R. E., Jóhannesson, G., Jackson, M. S., Jogler, T., Johnson, A. S., Johnson, W. N., Kataoka, J., Kerr, M., Knödlseder, J., Kuss, M., Lande, J., Larsson, S., Latronico, L., Lemoine-Goumard, M., Longo, F., Loparco, F., Lovellette, M. N., Lubrano, P., Massaro, F., Mayer, M., Mazziotta, M. N., McEnery, J. E., Mehault, J., Michelson, P. F., Mitthumsiri, W., Mizuno, T., Monzani, M. E., Morselli, A., Moskalenko, I. V., Murgia, S., Nakamori, T., Nemmen, R., Nuss, E., Ohno, M., Ohsugi, T., Omodei, N., Orienti, M., Orlando, E., de Palma, F., Paneque, D., Perkins, J. S., Piron, F., Pivato, G., Porter, T. A., Rainò, S., Rando, R., Ray, P. S., Razzano, M., Reimer, A., Reimer, O., Reposeur, T., Ritz, S., Romani, R. W., Romoli, C., Sanchez, D. A., Parkinson, P. M. S., Schulz, A., Sgrò, C., do Couto e Silva, E., Siskind, E. J., Smith, D. A., Spandre, G., Spinelli, P., Suson, D. J., Takahashi, H., Tanaka, T., Thayer, J. B., Thayer, J. G., Thompson, D. J., Tibaldo, L., Tinivella, M., Troja, E., Usher, T. L., Vandenbroucke, J., Vasileiou, V., Vianello, G., Vitale, V., Waite, A. P., Winer, B. L., Wood, K. S., Wood, M., Yang, Z., \& Zimmer, S. 2012b, Science, 338, 1314

Ransom, S. M. 2001, PhD thesis, Harvard University

Ransom, S. M., Eikenberry, S. S., \& Middleditch, J. 2002, AJ, 124, 1788

Ransom, S. M., Greenhill, L. J., Herrnstein, J. R., Manchester, R. N., Camilo, F., Eikenberry, S. S., \& Lyne, A. G. 2001, ApJ, 546, L25

Ransom, S. M., Hessels, J. W. T., Stairs, I. H., Freire, P. C. C., Camilo, F., Kaspi, V. M., \& Kaplan, D. L. 2005, Science, 307, 892

Ransom, S. M., Ray, P. S., Camilo, F., Roberts, M. S. E., Çelik, Ö., Wolff, M. T., Cheung, C. C., et al. 2011, ApJ, 727, L16 
Ransom, S. M., Stairs, I. H., Archibald, A. M., Hessels, J. W. T., Kaplan, D. L., van Kerkwijk, M. H., Boyles, J., Deller, A. T., Chatterjee, S., Schechtman-Rook, A., Berndsen, A., Lynch, R. S., Lorimer, D. R., Karako-Argaman, C., Kaspi, V. M., Kondratiev, V. I., McLaughlin, M. A., van Leeuwen, J., Rosen, R., Roberts, M. S. E., \& Stovall, K. 2014, Nature, 505, 520

Rappaport, S., Podsiadlowski, P., Joss, P. C., Di Stefano, R., \& Han, Z. 1995, MNRAS, 273, 731

Ray, P. S., Abdo, A. A., Parent, D., Bhattacharya, D., Bhattacharyya, B., Camilo, F., Cognard, I., et al. 2012, ArXiv e-prints, 1205.3089

Ray, P. S., Kerr, M., Parent, D., Abdo, A. A., Guillemot, L., Ransom, S. M., Rea, N., et al. 2011, ApJS, 194, 17

Reid, M. J., Menten, K. M., Zheng, X. W., Brunthaler, A., Moscadelli, L., Xu, Y., Zhang, B., Sato, M., Honma, M., Hirota, T., Hachisuka, K., Choi, Y. K., Moellenbrock, G. A., \& Bartkiewicz, A. 2009, ApJ, 700, 137

Roberts, M. S. E. 2002, ArXiv Astrophysics e-prints, astro-ph/0212080

Roberts, M. S. E. 2013, in IAU Symposium, Vol. 291, IAU Symposium, ed. J. van Leeuwen, 127-132

Roberts, M. S. E., McLaughlin, M. A., Gentile, P. A., Ray, P. S., Ransom, S. M., \& Hessels, J. W. T. 2015, ArXiv e-prints, 1502.07208

Saz Parkinson, P. M., Dormody, M., Ziegler, M., Ray, P. S., Abdo, A. A., Ballet, J., Baring, M. G., Belfiore, A., Burnett, T. H., Caliandro, G. A., Camilo, F., Caraveo, P. A., de Luca, A., Ferrara, E. C., Freire, P. C. C., Grove, J. E., Gwon, 
C., Harding, A. K., Johnson, R. P., Johnson, T. J., Johnston, S., Keith, M., Kerr, M., Knödlseder, J., Makeev, A., Marelli, M., Michelson, P. F., Parent, D., Ransom, S. M., Reimer, O., Romani, R. W., Smith, D. A., Thompson, D. J., Watters, K., Weltevrede, P., Wolff, M. T., \& Wood, K. S. 2010, ApJ, 725, 571

Shklovskii, I. S. 1970, Soviet Ast., 13, 562

Smedley, S. L., Tout, C. A., Ferrario, L., \& Wickramasinghe, D. T. 2014, MNRAS, 437,2217

Stairs, I. H., Faulkner, A. J., Lyne, A. G., Kramer, M., Lorimer, D. R., McLaughlin, M. A., Manchester, R. N., Hobbs, G. B., Camilo, F., Possenti, A., Burgay, M., D’Amico, N., Freire, P. C., \& Gregory, P. C. 2005, ApJ, 632, 1060

Stappers, B. W., Archibald, A. M., Hessels, J. W. T., Bassa, C. G., Bogdanov, S., Janssen, G. H., Kaspi, V. M., Lyne, A. G., Patruno, A., Tendulkar, S., Hill, A. B., \& Glanzman, T. 2014, ApJ, 790, 39

Stappers, B. W., Bailes, M., Lyne, A. G., Manchester, R. N., D’Amico, N., Tauris, T. M., Lorimer, D. R., Johnston, S., \& Sandhu, J. S. 1996, ApJ, 465, L119

Tauris, T. M. \& Savonije, G. J. 1999, A\&A, 350, 928

Tauris, T. M. \& van den Heuvel, E. P. J. 2014, ApJ, 781, L13

The NANOGrav Collaboration, Arzoumanian, Z., Brazier, A., Burke-Spolaor, S., Chamberlin, S., Chatterjee, S., Christy, B., Cordes, J. M., Cornish, N., Crowter, K., Demorest, P. B., Dolch, T., Ellis, J. A., Ferdman, R. D., Fonseca, E., GarverDaniels, N., Gonzalez, M. E., Jenet, F. A., Jones, G., Jones, M. L., Kaspi, V. M., Koop, M., Lam, M. T., Lazio, T. J. W., Levin, L., Lommen, A. N., Lorimer, D. R., 
Luo, J., Lynch, R. S., Madison, D., McLaughlin, M. A., McWilliams, S. T., Nice, D. J., Palliyaguru, N., Pennucci, T. T., Ransom, S. M., Siemens, X., Stairs, I. H., Stinebring, D. R., Stovall, K., Swiggum, J. K., Vallisneri, M., van Haasteren, R., Wang, Y., \& Zhu, W. 2015, ApJ, 813, 65

Thompson, C., Blandford, R., Evans, C., \& Phinney, E. 1994, The Astrophysical Journal, 422, 304

Thompson, D. J. 2008, Reports on Progress in Physics, 71, 116901

van Kerkwijk, M. H., Bassa, C. G., Jacoby, B. A., \& Jonker, P. G. 2005, in Astronomical Society of the Pacific Conference Series, Vol. 328, Binary Radio Pulsars, ed. F. A. Rasio \& I. H. Stairs, 357

Venter, C., Harding, A. K., \& Guillemot, L. 2009, ApJ, 707, 800

Venter, C., Johnson, T. J., \& Harding, A. K. 2012, ApJ, 744, 34

Verbiest, J. P. W., Bailes, M., van Straten, W., Hobbs, G. B., Edwards, R. T., Manchester, R. N., Bhat, N. D. R., et al. 2008, ApJ, 679, 675

Viganò, D., Torres, D. F., Hirotani, K., \& Pessah, M. E. 2015, MNRAS, 447, 2649

Webbink, R. F., Rappaport, S., \& Savonije, G. J. 1983, ApJ, 270, 678

Weisberg, J. M. \& Taylor, J. H. 2005, in Astronomical Society of the Pacific Conference Series, Vol. 328, Binary Radio Pulsars, ed. F. A. Rasio \& I. H. Stairs, 25

Wolszczan, A. \& Frail, D. A. 1992, Nature, 355, 145

Xing, Y. \& Wang, Z. 2015, ApJ, 804, L33 